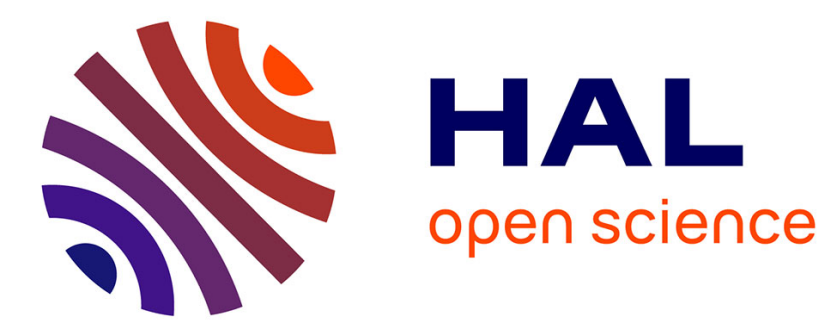

\title{
Revue européenne des migrations internationales
}

\author{
Gaëlle Lacaze, Dalia Rahmonova-Schwarz, Stéphane de Tapia
}

\section{To cite this version:}

Gaëlle Lacaze, Dalia Rahmonova-Schwarz, Stéphane de Tapia (Dir.). Revue européenne des migrations internationales: Migrations en Asie centrale et au Caucase. MIGRINTER. 26 (3), 2010, 978-2-91162756-9. hal-01334766

\section{HAL Id: hal-01334766 https://hal.science/hal-01334766}

Submitted on 21 Jun 2016

HAL is a multi-disciplinary open access archive for the deposit and dissemination of scientific research documents, whether they are published or not. The documents may come from teaching and research institutions in France or abroad, or from public or private research centers.
L'archive ouverte pluridisciplinaire HAL, est destinée au dépôt et à la diffusion de documents scientifiques de niveau recherche, publiés ou non, émanant des établissements d'enseignement et de recherche français ou étrangers, des laboratoires publics ou privés.

\section{(이) $\$$}

Distributed under a Creative Commons Attribution - NonCommercial - NoDerivatives| 4.0 


\title{
Revue européenne des migrations internationales
}

vol. $26-\mathrm{n}^{\circ} 3$ (2010)

Migrations en Asie centrale et au Caucase

Gaëlle Lacaze, Delia Rahmonova-Schwarz et Stéphane de Tapia

\section{Éditorial}

\begin{abstract}
Avertissement
Le contenu de ce site relève de la législation française sur la propriété intellectuelle et est la propriété exclusive de l'éditeur.

Les œuvres figurant sur ce site peuvent être consultées et reproduites sur un support papier ou numérique sous réserve qu'elles soient strictement réservées à un usage soit personnel, soit scientifique ou pédagogique excluant toute exploitation commerciale. La reproduction devra obligatoirement mentionner l'éditeur, le nom de la revue, l'auteur et la référence du document.

Toute autre reproduction est interdite sauf accord préalable de l'éditeur, en dehors des cas prévus par la législation en vigueur en France.
\end{abstract}

\section{revues.org}

Revues.org est un portail de revues en sciences humaines et sociales développé par le Cléo, Centre pour l'édition électronique ouverte (CNRS, EHESS, UP, UAPV).

\section{Référence électronique}

Gaëlle Lacaze, Delia Rahmonova-Schwarz et Stéphane de Tapia, «Éditorial », Revue européenne des migrations internationales [En ligne], vol. $26-n^{\circ} 3$ | 2010, mis en ligne le 01 décembre 2013, consulté le 08 février 2014. URL: http://remi.revues.org/5194

Éditeur : Université de Poitiers

http://remi.revues.org

http://www.revues.org

Document accessible en ligne sur : http://remi.revues.org/5194

Ce document est le fac-similé de l'édition papier.

(c) Université de Poitiers 


\section{Éditorial}

T es transformations des États de l'ancien bloc socialiste ont depuis vingt

Lans profondément modifié les économies locales, les modes de vie et enfin les relations régionales et internationales, participant ainsi à l'essor des migrations des peuples d'Asie centrale qui ont su mettre à profit l'ouverture des frontières internationales de l'ancienne URSS. Aujourd'hui, aux mouvements d'exode rural et aux migrations infrarégionales viennent s'ajouter des migrations vers la Russie, l'Europe ou d'autres pays riches comme la Corée du Sud. Le début du XXIe siècle voit, ainsi, s'accroître la mobilité des populations centrasiatiques.

L'existence de diasporas anciennes, qui reposaient sur une organisation sociale et des structures administratives héritées des politiques tsariste et soviétique, n'est pas sans lien avec les migrations contemporaines. Elles ont construit des réseaux circulatoires en « archipel », disponibles pour de nouveaux migrants et qui peuvent se déployer en - et hors - Asie centrale. On rappellera que les migrants centrasiatiques sont souvent de cultures pastorales nomades (Kazakhstan, Kirghizie, Turkménistan, Mongolie, en particulier). Cet héritage n'est pas là encore sans lien avec certaines formes que prennent les migrations aujourd'hui alors que les migrants tentent de s'adapter aux contraintes économiques et mobilisent des ressources propices aux déplacements. Et si certains pays offrent une destination migratoire préférentielle pour des « co-ethniques » de pays voisins, l'ensemble des processus à l'œuvre restructure les espaces infrarégionaux et remodèle la composition des groupes et des identités locales. Les migrations dans ce vaste espace centrasiatique apparaissent de plus en plus comme révélatrices des recompositions politiques, économiques et sociales qui caractérisent les anciens pays socialistes.

Ce numéro rassemble des articles de chercheurs centrasiatiques et européens, spécialistes de ces régions et des migrations. Les auteurs, politologues, historiens, anthropologues et géographes, rendent compte des recherches qu'ils mènent et rendent accessibles des données de terrain inédites. Chaque contribution aborde un aspect particulier des migrations en Asie centrale.

En guise de prologue, l'article de Delia Rahmonova-Schwarz illustre l'ancienneté des mouvements migratoires depuis ou en direction de l'Asie centrale et met l'accent dans ses analyses sur les migrations forcées et les restrictions migratoires à l'époque soviétique. La première partie de ce numéro regroupe des articles consacrés aux processus migratoires en Ouzbékistan. L'article de Julien Thorez rend compte des migrations de travail des Ouzbeks à différentes échelles, nationales, infrarégionales et internationales. Il met en évidence les nouvelles territorialités qui se dessinent en Ouzbékistan. Sophie Massot montre les changements sociaux induits par la migration, généralement des hommes, et les profonds changements qui s'opèrent au moment du retour au pays.

La seconde partie de ce numéro porte plus directement sur les migrations au Kazakhstan, en Kirghizie et au Tadjikistan. Amandine Regamey et Katherine Booth examinent la question sous l'angle des droits de l'homme dans la gestion politique des flux migratoires en Kirghizie et au Kazakhstan. Saodat Olimova interroge l'impact des migrations sur le développement des pays d'Asie centrale à travers l'exemple tadjik, déjà 
caractérisé par des flux migratoires importants à l'époque soviétique.

La troisième partie aborde le terrain caucasien, Azerbaïdjan et Géorgie, mais aussi les relations entre Kazakhstan et populations « co-ethniques » vivant à l'étranger. Sergey Rumyansev s'attache à montrer comment l'édification d'une diaspora ethnonationale, le « cas azéri », explique les différentes étapes d'élaboration d'une politique nationaliste en Azerbaïdjan. Gulnara Mendikulova compare la situation des Kazakhs de la diaspora avec celle des co-ethniques vivant à l'étranger, dits ici irredenta, à la suite de l'appel à la "re-kazakhisation » du pays. Ce texte illustre une « école » très présente dans la recherche actuelle dans la région, perspective que Sergey Rumyansev comme Gaëlle Lacaze n'adoptent pas ; cette dernière développe une analyse critique des stratégies des Kazakhs de Mongolie qui multiplient les mouvements d'aller-retour au Kazakhstan. MarcAntoine Pérouse de Montclos montre, quant à lui, l'utilisation politique des déplacements de populations après la guerre de 2008 en Géorgie. Sur un registre différent, Bayram Balci et Stéphane de Tapia traitent de l'investissement religieux de la Turquie en direction de l'Asie centrale où un syncrétisme musulman, turc et centrasiatique, tente de se développer.

Ces différentes visions des migrations, parfois en forte opposition, offrent aux lecteurs des approches comparatives sur des processus migratoires qui caractérisent aujourd'hui les anciens pays socialistes. Au cœur des recompositions politiques, économiques et sociales actuelles, les positions de chercheurs centrasiatiques donnent des clés pour aborder et comprendre l'importante et épineuse question des problématiques identitaires dans ces régions, où les mouvements migratoires en constituent une dimension significative. Les analyses de Gulnara Mendikulova et de Sergey Rumyansev portent sur les transformations idéologiques, à partir de points de vue très opposés, qui caractérisent aujourd'hui ces régions comme la plupart des pays issus de l'ancienne URSS. Extérieurs à ces problématiques qui traversent et agitent ces États nouvellement indépendants, les chercheurs occidentaux nous livrent leurs analyses qui portent plutôt sur les aspects formels et les pratiques migratoires. Ainsi l'article de Julien Thorez et celui d'Amandine Regamey et Katherine Booth privilégient un point de vue administratif et institutionnel. Ceux de Sophie Massot, de Bayram Balci et de Stéphane de Tapia, mettent plus l'accent sur les aspects idéologiques et symboliques des migrations centrasiatiques.

La diversité et l'originalité des points de vue ainsi que la pluralité des configurations migratoires présentées dans ce numéro illustrent la complexité des processus migratoires dont la régulation apparaît aujourd'hui comme l'un des enjeux centraux des politiques nationales des pays postsocialistes, des coordinations infrarégionales centrasiatiques et de leurs coopérations internationales. On pourra enfin se référer à la note de lecture sur l'ouvrage édité par Marlène Laruelle qui souligne combien cette région, autrefois considérée comme « immobile », est devenue le théâtre et l'origine de mobilités.

Gaëlle LACAZE, Delia RAHMONOVA-SCHWARZ et Stéphane de TAPIA 


\title{
Migrations during the Soviet Period and in the Early Years of USSR's Dissolution: A Focus on Central Asia
}

\section{Delia RAHMONOVA-SCHWARZ ${ }^{1}$}

\begin{abstract}
Central Asia is a region with strong migratory dynamics. Migration patterns from Central Asia have their roots in the policies of the Soviet state on mobility and labor. As this volume comprises a number of interesting country-specific cases, it is important to bear in mind that the region as a whole has for the past century witnessed various flows of in - and out - migrations including state-forced resettlements during the Soviet people. The present article has the objective to describe on the one hand state-forced migration movements during the Soviet period, and on the other hand, the opportunities for and restrictions on mobility that influenced the migration patterns during this time. These migration-related historical developments should be drawn to the attention of scholars studying the current migration flows within the region as well as between the region as the place of origin and other countries of migrant destination.
\end{abstract}

Central Asia is also home to people who represent various ethnic groups and languages. For example, there are ethnic Uzbeks, Russians, Koreans and Tatars in Kyrgyzstan or Tajikistan or a Tajik-speaking population in some parts of Uzbekistan, ethnic Russians, Uigurs and many other minorities in Kazakhstan, Uzbeks and Russians in Turkmenistan. Scholars dealing with migration from Central Asia to Russia, a major point of destination, to Kazakhstan or beyond the post-Soviet territory are often confronted with this ethnic heterogeneity. This article also sheds light on movements of people in the context of state nationality policies during the Soviet period. Finally, this contribution shows that labor-induced migration as seen on the post-Soviet territory had begun prior to the dissolution of the USSR.

1 Policy Support Officer, OSCE Conflict Prevention Centre, Wallnerstrasse 6, 1010 Vienna, Austria; schwarzdelia@web.de 


\section{THE EARLY YEARS OF MOBILITY CONTROL IN PRE-SOVIET RUSSIA}

Any person travelling nowadays to Russia or to some countries of the former Soviet Union has been confronted with a mandatory registration of residence, known in Russian as propiska or registraciia. Researchers of post-Soviet migrations have without a doubt heard from both internal migrants within Central Asian countries or in the Russian Federation about their difficulties obtaining legal documents of which propiska is an important part. Resident registration has an interesting history. Some scholars hold Tsar Peter the Great responsible for the initial concept of registering population in order to engage more recruitees for his reformed army, collect taxes, and rule with more law and order than his predecessors (Garcelon, 2001: 90). Others argue that as early as the end of the $16^{\text {th }}$ century the tsars kept a systematic registration of peasants who were bound to work for the feudal lords (Matthews, 1993: 1).

Under the regulations of the Russian tsars, the individual choice for mobility, particularly among the peasants, was limited. Movements of people took place for commercial or professional reasons whereas personal travels were highly restricted. However, subjects of higher ranks such as nobles and higher military personnel were issued permanent passports and enjoyed freedom of movement. The lower the rank, the more constrained the subjects were in their mobility. In cities, especially in St. Petersburg and Moscow, the subjects who were registered temporarily received address tickets that they were demanded to retain until their departure (Matthews, 1993: 10-12).

By the mid-19 ${ }^{\text {th }}$ century, strict control over population mobility became a hindrance as the demand for blue collar manpower increased together with industrialization of the urban areas. Issuance of passports became expensive for the impoverished, and consequently residents became increasingly reluctant to abide by the rules. In 1894, when the Statute on Residence Certificates was adopted under Alexander III, it was no longer demanded that urban residents obtain a permanent permit. When Central Asia was annexed to the Russian Empire in the 1860s, the statute loosened control over travels of subjects even more.

\section{CONTROL OF MOVEMENT THROUGH LABOR IN THE PERIOD AFTER THE 1917 REVOLUTION}

After the toppling of the tsarist regime and the establishment of the Bolshevik power, management of migration, settlement, and employment gained importance in the newly founded socialist state's policies towards forming a large multiethnic state - one in which every member would work to contribute to the favor of the socialist society. Following the October revolution, Bolsheviks who had been vigorously criticizing and intending to abolish the tsars' oppressive policies towards the exploited class soon established full control over the entire population with the same firmness. What is remarkable is that with a similar attitude as the tsars had had towards peasants whose movement they had restricted, the Bolsheviks demanded that all former members of bourgeoisie and upper 
classes obtain residence registrations. In doing so, the Bolsheviks compelled the former "exploiters" to participate in the military service and socially beneficial labor such as construction and maintenance. Holders of registration cards, who were adult men aged 18 to 45 , were obliged to have their employment information entered into their documents by their employers and trade unions. This procedure was followed by the policy of "labor books" implemented in October 1918 (Matthews, 1993: 16-19). The regulation initially applied to all people who had previously belonged to upper classes. Labor books were to substitute identity documents and passports. They contained personal data and all information on jobs assumed by the holder, who had to update it on a monthly basis at the local administrative or police offices. Most importantly, labor books had a special entry on the holder's place of residence (Matthews, 1993: 16-19). Propiska, the term for "residence registration" that emerged initially as an entry in these labor books, was a tit-for-tat instrument of control in the onset of the Bolshevik power. This practice would survive decades of state migration policies during and after the Soviet period and would be widely used in the ex-Soviet countries.

Shortly after the introduction of "labor books" for members of the formerly upper class, in December 1918 RSFSR employment legislation stipulated that every workable individual acquire a "worker's book" (rabochaia knizhka), a document which would include information on the place of employment, hours of service, salaries, vacations, sick leaves, maternal leaves, rewards, promotions, and penalties. As of February 22, 1922, any employment and/or leave of a worker involved permission from the local labor officials. Until recently in the years after the fall of the Soviet Union, a similar document called "trudovaia knizhka" (work book) served as evidence for inter alia, determining years of work to calculate pension rates. Between 1924 and 1925, after a one-year period of liberalization of regulations concerning population movements, a legislation was adopted which stated that any person assuming residence for at least three days must register with the landlords, administrator of the residence, or a hotel. Individuals receiving visitors had to register them with a law enforcement office within 48 hours upon their arrival. ${ }^{2}$ Details about the person were to be entered into the "house book" (domovaia kniga) (Matthews, 1993: 20; Buckley, 1995: 902). This book was to be kept at every abode with a detailed record of its residents; it too maintained its significance until the $21^{\text {st }}$ century in each of the four countries involved in labor migration.

\section{MANAGING MIGRATION AND INSTITUTIONALIZING RESTRICTION ON MOVEMENT OF PEOPLE UNDER STALIN}

When considering the institutional constraints imposed on the movements of people during the Soviet period, it is important to keep in mind the Soviet state's construction of nationalities in the 1920s and 1930s. It is during this period that allocation of geographical territories of the Soviet republics took place. One of the most important historical milestones is the year 1922, which marked the establishment of the Soviet

2 According to the Federal Law on Migration Registration of Foreign Citizens and Stateless Persons of the Russian Federation adopted in January 2007, the host party is required to notify the Federal Migration Service about the arrival of a foreign visitor. 
power in Turkestan - the region which later became known as Central Asia - as well as the foundation of the Union of the Soviet Socialist Republics (USSR). Two years later, in 1924, when the delimitation of administrative borders in Central Asia resulted in the creation of the Turkmen SSR and the Uzbek SSR, the latter first included the Tajik ASSR into its territory. Tajik ASSR then became Tajik SSR in 1929. Kazakhstan was known as Kazakh Autonomous Soviet Socialist Republic of the RSFSR. To RSFSR belonged also Kara-Kyrgyz Autonomous Oblast' (Province). In 1936, these administrative unites were renamed Kazakh SSR and Kyrgyz SSR, respectively.

In the mid 1920s, the Soviets launched their first population census. For this purpose, they engaged ethnographers who had been working for the Commission for the Study of the Tribal Composition of the Population of Russia (Hirsch, 2005). Prior to this period, the ethnographers' primary task had been to gain ethnographic data for the Russian Empire during the First World War. Following the October revolution, when they became consultants to the Bolsheviks, they were committed to all-Union censuses and participated in the activities that involved defining the internal frontiers of the Soviet Union. The Russian ethnographers carried out field research in remote areas to conduct in-depth study of different peoples of the USSR. However, as Hirsch argues, they did far more than merely accumulate data in the remotest places of the USSR; they contributed to a change in population composition by applying an approach that Hirsch terms as "state-sponsored evolutionism." According to her, the state, which was marked by a strong Leninist school of thought, envisioned the evolution of the population of the USSR. The Soviets, adhering to the Marxist ideology of development, held that it was feasible to expedite the evolution process by forming nationalities through clustering clans and tribes which in turn were deemed to be residuals of the feudal period (Edgar, 2004; Hirsch, 2005). ${ }^{3}$ Nationalities would be formed into socialist-era nations, which some day in the era of communism would all be united. In Lubin's words, "a 'flourishing of nations' was to lead to their eventual 'coming together,' or 'rapprochement,' which was ultimately to lead to their 'merging' or assimilation." (Lubin, 1984: 6). ${ }^{4}$ Thus, in compliance with the government's official list of nationalities, citizens had to choose or declare themselves as belonging to an official nationality during individual encounters with census-takers (Hirsch, 2005: 9).

A further relevant aspect for the study of post-Soviet migration is the principle of "double assimilation" applied by the Soviet regime. The characteristic of this kind of assimilation is the co-occurrence of top-down and bottom-up processes, which meant that not only did the Soviet government implement measures from above such as delineating national borders and publishing an official list of nationalities, but also that local elites were involved in the decision making and had to fight for resources, land, and power. Archival materials bear witness to the fact that even smaller population groups such as

3 Hirsch's elaborate work leaves an impression that the creation of ethnicities/nationalities in Central Asia occurred mainly beginning with the formation of the USSR, particularly under Stalin. Earlier scholarship on Central Asia points out that already in the $19^{\text {th }}$ century the tsars had the tendency to perceive of "people" by the language spoken by people (Volk). This German romanticism was then passed onto Marxists who advanced their own vision of constructing nations following the revolution in 1917 (Roy, 2000: 54).

4 More on "merging" (sliianie) and "coming together" (sblizhenie) of nations, see also Smith (1996: 8-9). 
peasants protested and complained to higher authorities about inappropriate distribution of their territories to the national territorial units they desired not to be part of (Hirsch, 2005; Edgar, 2004: 53, 60-61). ${ }^{5}$

Prior to the formation of the Soviet Union and the enhancement of the state nationality policies, the sense of identification with which Central Asian people associated themselves was based on their language, clan, tribe, geographic place, religion, and religious and/or noble ancestry (Roy, 2000: 48-60; Edgar, 2004: 18; Hirsch, 2005: 110-114). The border delimitation and the first all-Union census of 1926 gradually altered people's sense of self-identification, as the Soviet republics in Central Asia would include national majorities and minorities. Roy points out that the delimitation of these borders did not have any geographic, economic, or ethnic rationality. This resulted, as he rightly points out, in the emergence of such complex constellations of peoples as the large Uzbek population for which the Province of Osh in the south of Kyrgyzstan is known, or the ethnic Uzbeks living in Tashauz and Charjau (now Türkmenabad) in Turkmenistan as well as Shymkent and Jambul in Kazakhstan. In Tajikistan, the Uzbek speaking population is spread primarily in the north in the Sughd Province and the region of Hissor as well as some villages in the Province of Khatlon in the south. Similarly, in Uzbekistan, there is a large Tajik-speaking population in Buxoro, Samarqand, Surxondaryo, and even in the Ferghana Valley and the north of Tashkent. There are ethnic Kyrgyz in the north and south of Tajikistan (Roy, 2000: 116-118). ${ }^{6}$

In the midst of the border demarcation process and creation of nations, the party-state was especially responsible for controlling rural out-migration and managing mobility of the population. The establishment of the Soviet power resulted in emigration of thousands of people who opposed the communists. In the Central Asian republics, the former upper class people were marginalized and driven from urban to rural areas. The formation of socialist collective farms led thousands of better-off peasants to flee from the Soviet regime into exile. Particularly in the 1930s, the so-called kulaks, or rich peasants/entrepreneurs, were taken under state scrutiny. Repression and execution of the kulaks forced a great number of people to seek refuge in Afghanistan, Iran, China, Turkey, Saudi Arabia, and in Western European countries (Kocaoğlu, 2000). In nomadic regions of Turkmenistan, Uzbekistan, Kyrgyzstan, and Kazakhstan, collectivization was used as a tool to bring about a sedentary lifestyle. As Edgar argues, nomads were perceived by the Soviet administration as people difficult to control, who were opposed to the Soviet power

5 Edgar points out interesting examples from the 1920s in which Soviet ethnographers were unable to determine the nationality of certain nomadic tribes based on their languages. There were cases in which the Soviet authorities found it extremely difficult to distribute certain territories during the border delimitation process.

6 I refrain from referring to the Tajik-speaking population as Tajiks and the Uzbek-speaking population as Uzbeks. The Soviet citizenship passport had a special nationality entry. Following the demise of the USSR, when the citizenship passports in each Central Asian country were introduced, the citizens in Central Asian countries were once again confused. Either the citizenship passports did not have such an entry (Uzbekistan) or representatives of ethnic/linguistic minorities were categorized according to their place of birth, as in the case of Tajiks who were registered as Uzbeks since they had been born in Buxoro in the 1940s and moved to Tajikistan during the Soviet era. They do not necessarily speak Uzbek. 
and prone to cross the Soviet border to flee. According to Soviet understanding, only those who possessed a great deal of wealth were able to afford to lead a nomadic life, as any change of place of residence with family and household would require considerable means (Edgar, 2004: 195).

The first Five-year Plan which was launched in 1928 marked a head start towards the "modernization of culturally backward people", sedentarization, and enhancement of development through industrialization and collectivization of agriculture (Edgar, 2004: 88; Collins, 2006: 85). ${ }^{7}$ At the same time, the Soviets implemented a policy of distribution of labor that they coupled with strict migration control. It is in this context that the Central Asia region started receiving labor migrants from Russia through orgnabors, an organized system of labor force recruitment for the mining industry, railway and motorway construction, technical personnel for construction of factories and plants, etc. (Shigabdinov and Nikitenko, 2000: 104).

In 1932, Stalin issued a decree according to which internal passports were to be issued - allegedly - not only in order to gain more precise statistical information from urban areas, but also "in order to secure the deportation from these places of persons who are not connected with industry or with work in offices and schools, and who are not engaged in socially useful labor (with the exception of infirm persons and pensioners), and also in order to cleanse these places of kulak, criminal and other anti-social elements finding refuge there..." (Matthews, 1993: 28).

The decree applied to any individual older than 16 years residing in border zones, urban areas, or towns (or within $100 \mathrm{~km}$ around them). ${ }^{8}$ Four months after this legislation was issued, it was complemented by a further decree determining a list of 25 towns and $100 \mathrm{~km}$ radiuses to which the decree would apply. All other individuals living beyond the officially designated "regime zones" were deprived of passports and were required to be registered with the rural district authorities. This meant that minority populations who had been allocated to reside in designated territories (army members and, particularly, peasants, i.e. collective farmers who constituted a majority among the population) were also Soviet citizens, but they had difficulty traveling and seeking residence and employment as they wished in the areas which were under strict control through internal passports. Without a passport it was impossible to travel to distant locations since boarding trains and buses required showing a valid internal passport. Only on a seasonal basis and with the permission of their supervisors were members of kolkhozes allowed to work in the spheres of industry and construction (Lewis and Rowland, 1979: 22). In addition, under the oppressive regime of Stalin, defiance of passport and registration rules was punished. Penalties varied from fines to a prison sentence of up to two years; forgery of documents was severely prosecuted (Lewis and Rowland, 1979: 22; Matthews, 1993: 28; Garcelon, 2001: 91).

7 Specifically on internal migration control and collectivization, see Garcelon (2001).

8 Hirsch mentions that the internal passport system of the USSR distinguished between three territorial categories: (1) "regime zones," which encompassed territories of geopolitical or economic importance that later would include surrounding regions within $100 \mathrm{~km}$ designated as such, as well as frontier regions that were located $100 \mathrm{~km}$ within the Soviet territories bordering the European and Far Eastern states, (2) "non-regime zones," the term applied to rural regions and provinces, and (3) "extra-administrative zones" that included the Gulag (2005: 275). 
In light of the Soviet efforts to develop industry and cotton monoculture in Central Asia, arid oases in the south were to be cultivated. Due to a great deficit of manpower in these parts of the region, groups of population were relocated involuntarily from the Ferghana Valley to these areas. During the period from 1929 until the mid-1950s, in accordance with Stalin's oppressive attitude toward kulaks and other 'undesired' minority population in Central Russia and Siberia, "special settlements" (spetsposelenie) were established in the region where these forced settlers had to reside (Shigabdinov and Nikitenko, 2000: 103). A typical example is the deportation of 171,781 Koreans to Kazak and Uzbek SSR in the late 1930s, which occurred due to increased Soviet suspicion of ethnic Koreans residing in the frontier territories of the Far East. The Soviets feared that ethnic Koreans might establish close ties to Korean nationals and Japanese intelligence agents with Korean nationality (Shigabdinov and Nikitenko, 2000: 104).

The period during World War II and the years immediately after witnessed, despite the strict control on movement, different migration flows including rural-to-urban movements due to the increase of industrialization and expansion of urban areas. Migration of Slavic population to Central Asia increased during and after World War II, partly related to the evacuation of population from Russian territories under the German occupation, but also as a result of the transfer of plants, construction of military infrastructure, provision of clothing and food supplies, and redistribution of resources during the war (Shigabdinov and Nikitenko, 2000: 104). Among the Central Asian republics, Kyrgyz SSR and Kazakh SSR had the largest inflow of Russian-speaking migrants in the decades after World War II (Islamov, 2000: 181-182).

The onset of World War II and the invasion of the Nazi troops marked the deportation of Germans from the Volga Region to Central Asia, their overall number in 1941-1942 amounting to 1,209,430. Forced relocation of minorities in the USSR was not confined only to Germans, however. The number of "special settlements" grew as Chechens, Ingushes, Crimean Tatars, Kurds and Greek political migrants were resettled forcefully into various regions of Central Asia (Polyan, 2001; Berdinskikh, 2005). For instance, a relatively large group of around 60,000 Meskhetian Turks was resettled from Georgia to the Ferghana Valley in Central Asia in 1944 (Rubin and Lubin, 2000: 177). Almost 40,000 Greeks were relocated from the Black Sea region, mainly to Kazakhstan and Uzbekistan during the same period (Bugai and Kotsonis, 1999: 55, 117).

Notwithstanding the adoption of strict policies such as the introduction of mandatory registration at the place of residence and the internal passport system, there seems to have been some difference between policy and practice. Lewis and Rowland as Buckley state that in reality, those wishing to change their place of residence succeeded in doing so by using informal channels. Lewis and Rowland argue that despite the common understanding in the West that Stalinist policies severely restricted mobility, one should contextualize the migration regulations of that time. The decline of the economy, extreme poverty, starvation and collectivization of the 1920s and 1930s led the population to move to urban areas. Between 1926 and 1939, as Soviet statistics states, 18.7 million people migrated from rural areas into urban areas (Lewis and Rowland, 1979: 25). In spite of the limitations imposed on the cities that were officially "closed" to migrants from outside through the notorious propiska (residence permit) regulation, many potential migrants 
sought different alternatives such as informal practices to attain their goals and eventually succeeded in settling. An elaborate study conducted by Buckley shows that in reality, the restrictive measures of the Soviets to control the vast growth of the cities were quite unsuccessful (Buckley, 1995).

\section{MIGRATION PATTERNS IN THE 1960'S AND 1980'S}

\section{The Presence of the Slavic Population in Central Asia}

During this period the Central Asian republics experienced a sharp increase of demographic growth. The population of the RSFSR slightly declined, with the exception of some industrial regions (Gibson, 1991: 152). The statistical data on the changes of residence of individuals within the USSR show that the population of the Central Asian republics remained relatively immobile, whereas in RSFSR people showed more mobility and moved between different regions (Lubin, 1984: 41; Gibson, 1991). For the period of 1959-1979, the Central Asian republics report the following data on the increase of the population among the titular nationalities: Uzbekistan (6.7\%), Kyrgyzstan (7.5\%), and Tajikistan (5.8\%) (Islamov, 2000: 182).

With Khrushchev's arrival to power in 1956 and his policy of de-Stalinization, control over workers' mobility was loosened. Through the orgnabors (the aforementioned organized labor recruiting service), the government continued to administer the distribution of labor force, relocating 28 million people in the period between 1930 and 1970. This organized recruitment was not mandatory, however. From 1951 until 1970, the number of resettled workers decreased, totaling merely 573,100 in the period of 1966-1970 (Lewis and Rowland, 1979: 19; Lubin, 1984: 42-43).

By the mid-1970s collective farmers that reached the age of 15 were permitted to obtain internal passports with which they were able to travel longer distances. The sources provided by Islamov (2000) suggest that in the time period of 1959-1979, Kyrgyzstan, Tajikistan, and Turkmenistan saw an increase of $13.1 \%, 8.5 \%$, and $10.9 \%$ of ethnic Russians, respectively. By 1970, around 30 million Russians were residing in areas primarily inhabited by non-Russians that were located beyond the administrative borders of the RSFSR. This number constituted 23 percent of the overall Russian population. Between 1979 and 1989, however, more Russians were reported to have left Central Asia than to have arrived (Shigabdinov and Nikitenko, 2000: 104). Gibson notes that the number of migrants from RSFSR to Central Asia dropped by 50\% during the period of 1971-1980 (Gibson, 1991: 153). The post-Stalinist period was marked by the redistribution of power and education and employment opportunities among Russian-speaking professionals and representatives of the so-called titular nationality. As argued by Korobkov (2008: 71), with regional governing bodies in Central Asia promoting more employment and education opportunities for the titular nationality, Russian speakers were pushed to take lower-level jobs which in turn led them to leave the region. 


\section{Emigration of the Jewish Population}

The period between late $1960 \mathrm{~s}$ and $1980 \mathrm{~s}$ is characterized by a large-scale emigration of Jews from the entire Soviet Union (including Central Asia). Prior to this period, the USSR had never before witnessed a mass permanent outflow of its citizens. In September 1968, the Embassy of the Netherlands in Moscow launched the issuance of exit permits that would allow Jews immigration to Israel (Buwalda, 1997: 9-11). ${ }^{9}$ What is more, it occurred at the time when very few citizens were given permission to travel to foreign countries. By 1971, the outflow of the Jewish minority increased sharply. Within this year, at least 13,000 people were allowed to leave. A year after, almost three times as many Jews left the USSR for Israel. Buwalda (1997) distinguishes between two waves within this exodus of the Soviet Jews. The first wave is in 1970s when 220,000 people emigrated. During this period the majority of Jewish emigrants left for Israel. Between 1975 and 1987, two thirds of the emigrants preferred to settle in countries other than Israel (Dietz, 2000: 639). The second wave started in 1987 and continued through 1991, with the number of Jewish emigrants amounting to 300,000 (Buwalda, 1997: 9-11).

The shift from strict controls on travel abroad to sweeping permission of such a great number of the population to emigrate in the midst of the Cold War seems remarkable. In this context, too, we should address the opportunities and constraints that shaped this pattern of migration. Zaslavsky and Brym (1983: 2-3, 64-76) discuss in their joint work the extrinsic and intrinsic modes in the scholarship on Jewish out-migration from the Soviet Union. The first of the two approaches links the outflow of the Jewish population to external factors occurring in the late 1960s and 1970s. The revival of the Jewish national consciousness and the strong desire of the Soviet Jews to emigrate to Israel is explained as an outcome of the Six Day War in 1967, in which Israel claimed victory, as well as the foundation of the state of Israel thereafter. In this context, it should not go unmentioned that the Kremlin was against Israel during the Six Day War, and this position had a major impact on the Jewish community all over the USSR. A further factor in this approach is the pressure which the West exerted on the USSR due to its desire that Jews leave the country. ${ }^{10}$

The second approach is related to social changes occurring within the confines of the USSR, and shifts the external factors to the background. The extent to which the Jewish out-migration took place is explained in terms of the Soviet preventive policy of a sudden deficit in labor force, particularly in the technical and scientific spheres. A further reason is argued to have been individual decision making: some had merely no desire to

9 The period of the late 1960s and early 1970s is considered by scholars such as Buwalda to be the second exodus of Jews. Buwalda points out that the first exodus of Jews took place in the period of 1881-1914 in which roughly 2 million Jews left the Russian Empire.

10 Zaslavsky and Brym provide some details about how Soviet-American trade agreements correlated with the attitude of the Kremlin's number of permissions for Jews to exit. Thus, in 1972-1973 when the wheat agreement was signed, between 32,000 and 35,000 emigrants were recorded. The Jackson-Vanik amendment, which warned that trade would be blocked if Jews were not permitted to leave, caused a decrease of exits. Further wheat accord resulted in an increase of emigration permissions, which then declined with the start of the Soviet military actions in Afghanistan in 1980 and the wheat embargo. 
abandon the society in which they had been brought up, whereas others were induced to emigrate by having heard the stories of their friends and relatives.

There are indeed a number of other push and pull factors which cannot be analyzed here. ${ }^{11}$ One of the most important and detrimental implications of the Jewish emigration from the Soviet Union has been brain-drain. It is worthwhile to point out this aspect, since the onset of brain-drain out of the Soviet Union became discernible in the republics of the Central Asian region that had been home to thousands of Jews throughout many centuries. ${ }^{12}$ It is difficult to determine precisely how many Jewish scientists, professionals, and skilled workers in total emigrated from Central Asia between 1970 and 1991. On the one hand, statistical data accumulation in the Soviet Union is a controversial topic in the sense that, in the context of anti-Semitic attitudes towards Jews, some individuals had chosen to identify themselves as belonging to different nationalities. On the other hand, in their quantitative data and discussions on the issue, some Western scholars (notably in the US) seem to have an anti-Soviet bias. What becomes clear is that Soviet authorities must have sensed the danger of brain-drain as a serious consequence of emigration when they imposed a so-called 'education tax' in August 1972.13 According to this regulation, every emigrant had to compensate the Soviet state for the free education that they had obtained before their departure. The cost of emigration was in some cases as high as five to ten years of wages of a professional employee (Zaslavsy and Brym, 1983: 64). Other sources state that the fee was 4,500 rubles for a graduate in the humanities and 19,400 rubles for a doctor of sciences. Towards the end of that same year, the education fee had to be abolished, as the Western countries and President Johnson of the US threatened the USSR with sanctions (Buwalda, 1997: 91).

Although Western scholars working on this subject claim that, due to the antiSemitism that was present during the Soviet Union, the overall percentage of Jews who obtained higher education was only $1 \%$ in the entire USSR, having lived in the vicinity of a Jewish district, I cannot confirm the accuracy of such a low percentage in Central Asia. What became easily noticeable throughout the last two decades of the Soviet era was the absence of Jewish academicians, university professors, teachers, medical doctors, and scientists. In addition to brain-drain, the emigration of Jews meant the ultimate loss of a culture which was unique in its own, that had been developed and passed on through generations. Inherent to it were architecture, artisanship, music, and art of which almost no traces are left, and to which scholars focusing on emigration generally tend to pay too little attention.

11 There is an entire body of research in and outside of the former Soviet Union that deals specifically with Jewish emigration from the Soviet Union, including topics such as the Stalinist attitude towards nationality policy (Hirsch, 2005; Zaslavsky and Brym, 1983), the rise of antiSemitism in Russia (Zaslavsky and Brym, 1983), persecution and discrimination of Jews in the USSR (Buwalda, 1997), Soviet Jewish immigrants in Israel (Berthomière, 2003), and the Jewish minority population that chose not to emigrate (Ryvkina, 1996).

12 According to the sources provided by Zaslavsky and Brym, the total number Jews in 1970 in Uzbekistan was 102,855; Tajikistan, 14,615; and in the Kyrgyz Republic, 7,680.

13 This is not to deny that there was certainly more than mere fear of brain-drain when the authorities in the USSR established education fees. The historical passage of movement control in the USSR that covers the period prior to the Jewish emigration shows that the authorities most probably intended to prevent emigration. 


\section{"Friendship of Nations" and Construction of a Multiethnic Soviet Society: Education Migration, Internationalization of the Division of Labor and Solidarity among Nations}

In 1966, an earthquake of 7.5 on the Richter scale devastated the city of Tashkent, the capital of Uzbek SSR. Immediately, it became a major incentive for the Soviet authorities to reconstruct a new modern city that would embody the Soviet rhetoric of the "friendship of nations". The Soviet urban planners took necessary advantage of the damage caused by this natural disaster. They wiped out the majority of the residential buildings in the old town of Tashkent. The reconstruction efforts involved construction of multi-storied concrete residential buildings, schools, and hospitals, as well as creation of new industries and measures to develop infrastructure. This resulted in the inflow of thousands of migrants, particularly from the Slavic regions of the USSR. Around 30,000 people are estimated to have settled yearly during the early 1970s, all of them set to design Tashkent as a multicultural city epitomizing the "friendship of nations" (Lubin, 1984: 42; Roman, 2002; Sahadeo, 2007).

What is interesting and has been neglected in the research is that immediately after the earthquake, some higher education institutions in Tashkent evacuated their students and transferred them to the RSFSR. Despite scarce research which has been done particularly on the issue of outmigration after the earthquake, empirical data for the present study has indicated that some students among this generation settled in their new locations in Russia permanently. During my field research I encountered a case in which one of the former students had meanwhile become an important person in a patronage network of Central Asian migrants.

Along with the Russification policies and the efforts to raise the level of education in the less-developed peripheries of the USSR such as the Caucasus and Central Asia, the late 1960s through early 1980s was a period during which the educated youth, often the children of the party cadres and education elites, went to Russia to study at higher education institutions. Many of them came back to their region of origin as expected and pursued promising careers; some, however, chose to settle in Russia. From 20042007, Sahadeo conducted a survey with this precise category of migrants who had lived in the cities of Leningrad and Moscow during the Soviet era, who serve as evidence for the existence of an entire generation of education migrants in Russia and who recount different attitudes they received in their Slavic host societies (Sahadeo, 2007).

Research conducted by Lubin in late 1970s, which focuses mainly on Soviet Uzbekistan, brings two further aspects into the foreground, whose are of great significance for the present study: firstly, according to Lubin, the Central Asian (specifically Uzbek SSR) workable population migrated to the RSFSR for vocational, professional, and educational purposes. Secondly, she mentions that the Soviet Army served as a channel through which male migrants from Central Asia were allocated to different Russian cities. Lubin states that no data had been published on those migrants who had chosen not to return to Uzbekistan and that the individuals she had interviewed showed strong willingness to return to Uzbekistan (Lubin, 1984: 43). However, we do know in the meantime that some migrants did opt to stay permanently in Russia. Indeed, census data indicates that in 1959, 
42,000 Uzbeks were residing outside the territory of the Central Asian region. In 1979 the number of such migrants doubled, but considering that the number of the Uzbeks in the entire USSR was 12.5 million, 91,000 residing outside Uzbek SSR, seems miniscule (Lubin, 1984: 43).

According to sources provided by Sahadeo, in 1970 140,000 Kyrgyz, Tajiks, Uzbeks, and Turkmens were registered as residents in Russia. This number increased to 248,000 in 1989 (Sahadeo, 2007: 563). The present study will show that precisely these types of pioneer migrants - originating from all Central Asian republics, arriving in the 1960s and 1970s and staying in Russia, meanwhile maintaining ties to their regions of origin - played a crucial role in forming labor migrant networks in the 1990s. As we shall see in the course of this study, these actors became significant propellers of the inflow of cheap labor force. Those whose ties to their homelands had been rather weak benefited from their resources in Russia, and established migrant organizations and recruitment offices or have acted as informal job brokers and supervisors. ${ }^{14}$

\section{Russia's First Central Asian Labor Migrants?}

Military service and education were not the sole purposes for Central Asians to migrate to Russia for longer terms. Contrary to the common impression that the current mass labor migration from the Central Asian republics of the former Soviet Union to the Russian Federation commenced in the late 1990s, sufficient data has been found that implies the phenomenon of labor force movement from Central Asia to Russia is not characteristic of the post-Soviet period. While I argue that the dynamics of labor migration has represented a new challenge to the newly independent states, there was a period not too long before 1991 when the Soviet state encouraged the able-bodied population of Central Asia to settle in the regions of Russia that had labor deficit for the sake of resource allocation and production.

During the 1970s together with urbanization and industrialization in the RSFSR, the party-state staged efforts to cultivate the virgin land to increase food supplies, which of course required labor force. Soviet demographers criticized and complained about the absolute reluctance of Central Asia's workable population to migrate to different regions of the USSR to work. They stated that it was firstly due to a lack of education of Central Asians and secondly because the regions in Russia with severe labor deficit were not appealing to Central Asians in cultural, social, and material terms. The third argument was that the potential migrants were fairly satisfied with the environment in which they were living, regardless of the economic challenges of the period (Wimbush and Ponomareff, 1979: 11).

14 Here, we should be cautious about developing the two abovementioned aspects into categories such as education/vocation and military migrants as one might be tempted to. Among Lubin's interviewees there were namely those who stayed to study in Russian after the military service, which can make clear distinctions difficult. Similar cases emerged during my own fieldwork in Russia from 2005-2008. 
It goes without saying that the vast mobility of labor from Central Asian countries to Russia since the end of 1990s described in this volume has refuted these statements. What should be made clear is that there is significant historical data about movements of people from Central Asia to RSFSR during the Soviet period. In the second half of the 1970s, the Soviets expanded their agricultural development projects in the grey and brown earth zones of Moscow and Novgorod (known as the non-black earth zone, Rus. nechernozem) that extend to the north and the east of Russia, the territory covering Murmansk and the Urals (Wimbush and Ponomareff, 1979). It was planned that by 1980, the non-black earth zone would produce one-sixth of the overall agricultural production of the whole USSR (Wimbush and Ponomareff, 1979: 18-19). Development of agriculture in the non-black earth zone, the lower Volga, and Siberia, as well the establishment of Baikal-Amur Railroad, entailed engaging manpower from the regions with more labor force (Lubin, 1984: 43, 256; Gibson, 1991: 149). In 1978 the first group consisting of 1,200 Uzbeks was sent, and a year later as many as 3,900 were sent to the non-black earth zone to contribute to the development of agriculture (Lubin, 1986: 43). While both Lubin and Gibson provide us with marginal information on this topic and treat it within a broad macro-level study, I was able to explore some details of this phenomenon from newspaper articles published in the early 1980s.

In an article concerning Uzbek workers who opted to stay in the non-black earth zone, the Tashkent newspaper Yosh Leninchi (Uzb. 'Young Leninist') published an interview with Uzbek workers of both Slavic and Uzbek origin who expressed their strong desire to stay in Novgorod, Russia, and work there "not temporarily, but permanently."15 Uzbek workers are quoted as promising that they will not go anywhere "until we turn the nonblack earth lands into a productive area...". According to another report, Uzbek workers, as their names suggest, meanwhile married local Russian women, founded families, and were provided with apartments (Sahadeo, 2007: 562). They did not return to Uzbekistan, even though their training had finished long ago. Uzbek migrants and their families are described as living a normal life. More weddings were planned to be celebrated the year after the report and concert ensembles from Uzbekistan were reported to be on tour in the Russian region.

Newspapers from other Central Asian republics attest that these projects were not limited to the Uzbek SSR. The newspaper Kommunist Tadzhikistana reported on May 15, 1983 that an entire train had left Dushanbe, the capital of Tajik SSR, for Khabarovsk, carrying 70 families or more than 300 people, whose average age was 30 . This train was the second to follow the first one that had left two months previously with 100 families. The report cited the train supervisor as saying that all the migrants [ $\mathrm{sic}]$ were bound to the Far East to live and were skilled people. Experienced drivers, crane truck operators, technicians, construction specialists, and engineers with previous professional experience specifically in land cultivation projects in Kazakhstan were being sponsored by the Soviet

15 Notes from the Nonchernozem Zone. Article by Gh. Shermuhamedov, in Yosh Leninchi, $25^{\text {th }}$ of March, 1983. United States. (1983). USSR, post report. Washington, D.C.: U.S. Dept. of State. The articles which I have been able to consult in the archives were in the form of short reviews translated from the original language. The reviews reflected relevant press articles published in the Kyrgyz, Tajik and Uzbek SSRs during the 1980s. The name Nonchernozem was translated as such from Uzbek into English. 
government to work and live in the Far East. G.D. Dzhavov, deputy chairman of the Tajik SSR State Committee on Labor, stated that within that five-year period, Tajikistan intended to send 6,000 workers to the Far East. The deputy chairman contended with pride that Tajikistan would do its own share in the projects of the Far East. ${ }^{16}$

Lubin's findings about Central Asian workers' definite plans to return to their native regions resulted from her fieldwork which she had carried out in the late 1970s, i.e. shortly after these projects started. We can see from sources dating to several years later though, that a considerable number of these migrants did opt to stay. The scarcity of data on Kyrgyzstan does not mean that its population was not affected by organized interregional labor. As far as this country is concerned, only one source mentions that in the 1980s approximately 30,000-40,000 workers were engaged from Kyrgyzstan in other Soviet republics, among their 6,000-9,000 are reported as students from the Kyrgyz SSR. They were involved mostly in construction sites, in groups known as stroiotriads (Abazov, 2000: 231).

A further aspect which is particularly relevant in the context of migration and labor is again the rhetoric of the "friendship of nations" with which these migrant workers were mobilized. Among the workers from Central Asia there were both ethnic Slavic and Central Asian nations, as the names of the persons suggest. What is really remarkable is the way the interviewees stress that they had been working in a multiethnic environment. As one engineer states, his group consisted of "representatives of 14 nationalities working selflessly." 17 Attention should be drawn also to the "group approach" with which the Soviet leadership mobilized the outmigration of Central Asians. By sending entire groups or collectives, as they were called, the leadership intended to facilitate the adjustment of these workers in the region of arrival. In 1976, according to the decree of the Central Committee of the CPSU, Uzbek SSR was commissioned to assign construction trusts or groups to carry out land reclamation work in Novgorodskaia and Ivanovskaia oblasts within the framework of the aforementioned non-black earth cultivation project. ${ }^{18}$ In Novgorod, for instance, the trust was called Uznovgorodstroi and had 2,500 members in 1979. In addition to irrigating and draining land and constructing houses, schools, and hospitals, the trusts had to found state farms and give them as gifts to their oblasts before

16 "Bon Voyage! Tadzhikistan to the Aid of the Country", L. Serebrennikov L., in Kommunist Tadzhikistana, 15th of May, 1983. United States. (1983). USSR, post report. Washington, DC: US Dept. of State.

17 Notes from the Nonchernozem Zone. Article by Gh. Shermuhamedov in Yosh Leninchi, $25^{\text {th }}$ of March, 1983. United States. (1983). USSR, post report. Washington, DC: US Dept. of State.

18 Construction "trusts" (in Russian trust) consist of a group of industrial or commercial enterprises with centralized direction (cf. Oxford Russian-English Dictionary, 1973). The official name of the resolution was "Ob obiazatel'stvax kollektivov vodoxoziaistvennykh i stroititel'nykh organizatsii Uzbekskoi SSR po okazaniiu pomoshchi Ivanovskoi i Novgorodskoi oblastiam v vypolnenii postanovleniia CK KPSS i Soveta Ministrov SSSR po dal'neishemu razvitiiu sel'skogo xoziaistva nechernozemnoi zony RSFSR." [On the obligations of groups belonging to organizations in charge of water management and construction of the Uzbek SSR in its assistance in Ivanovsk and Novgorod Provinces in the framework of the decree of the Central Committee of the CPSU on further development of agriculture in non-black zone of the RSFSR] (Wimbush and Ponomareff, 1979: 14, also Ref. 35). 
their departure, as it was planned to make the region a major supplier of vegetable, meat, and dairy products. The two state farms in Novgorod were proudly named Tashkent and Druzhba (Rus. "friendship").

These examples describe that, firstly, the Soviet state made efforts to design a multicultural society and promoted cooperation between different republics. Secondly, by creating facilities for Central Asian youths to study outside of their region of origin, especially in the RSFSR and by sending young recruitees from the region to serve in the Soviet Army, the 'merger of nations' was sponsored further. Thirdly, through shifting different skilled human resources between the regions within the USSR, the Soviet state supported its own development through migration strategy that should be addressed as characteristic in its own way. Ultimately, studies conducted by Sahadeo, Roman, and Hirsch contend that the Soviet ideology of internationalization privileged Russians over non-Russians and thus engendered more ethnic discrimination towards the non-Slavic population in Russia.

\section{MIGRATIONS IN THE LATE 1980'S AND THE DEMISE OF THE USSR: WITHER "FRIENDSHIP OF NATIONS" AND "BROTHERHOOD OF PEOPLES"?}

In June 1989, when the USSR was facing a major economic crisis, interethnic clashes took place in the Ferghana Valley of Uzbek SSR between Uzbeks and the Meskhetian Turks who had been resettled during the Stalinist period. A fight that started at a small market kindled major violence against Meskhetian Turks in the cities of Ferghna, Kokand, Marilan, and Namangan. According to official reports, 171 people died as a result of the violent clashes and the vast majority of them were Meskhetian Turks. (Rubin and Lubin, 2000: 45-46; Megoran, 2002: 243). As a reaction to these clashes, the Soviet government relocated almost all 15,000 Meskhetians out of Uzbekistan (Rubin and Lubin, 2000: 45-45, 177).

In the following month, in July 1989, ethnic clashes broke out between Tajiks and Kyrgyz in the Isfara Raion in the north of Tajikistan that borders with Kyrgyzstan. Around 5,000 ethnic Kyrgyz refugees from Tajikistan settled in the south of Kyrgyzstan (Smith, 1996: 397). A series of other inter-ethnic violence acts followed suit. ${ }^{19}$ In midFebruary in Dushanbe, the capital of Tajikistan, there was a failed attempt of a coup d'état. Opponents of Mahkamov, the First Secretary of the Supreme Soviet of the Tajik SSR, plotted it by spreading rumors that thousands of Armenians fleeing from the clashes in Nagorno-Karabakh would be provided with housing and employment. The riot which was triggered by these rumors cost the lives of 22 people. As a result, representatives of ethnic

19 To these events one can add the inter-ethnic clashes between Kyrgyz and ethnic Uzbeks in Southern Kyrgyzstan in June 1990. More on this in the context of economic crisis in Kyrgyzstan, see Abazov (1999). Not much research has been done however, with regard to the impact of 1990 inter-ethnic clashes on the out-migration ethnic Uzbeks. While the author is cognizant of the most recent June 2010 violent inter-ethnic clashes in Southern Kyrgyzstan, this topic cannot be treated in this article. Furthermore, it will have to be seen to what extent the population from the South, both ethnic Uzbeks and Kyrgyz will choose to migrate to other countries. 
minorities such as Russians, Germans, Armenians, and Bukharan Jews, a total number of about 100,000, left Tajikistan (Smith, 1996: 380). The same false rumors about Armenian refugees settling in Frunze (now Bishkek), Kyrgyzstan resulted in mass protests. Although the skirmishes in Bishkek did not have any consequences related to migration, the ideology of "friendship of nations" and "fraternity of peoples" which had been meant to construct a multiethnic society in the USSR was shattered.

In 1989, approximately 25.3 million Russians were residing beyond the borders of the RSFSR, which corresponded to $18.5 \%$ of all Russians living in the USSR (Zaionchkovskaya, 1999: 11). The late 1980s marked the period during which Gorbachev's liberal policies and reforms had corollaries such as the rise of national self-consciousness, the declaration of Central Asian republics of their respective titular language as the official language, and the replacement of Russian party cadres by representatives of the titular nations. The latest waves of the Jewish emigration became concatenated with the permanent move of the Russian speaking population toward the RSFSR or the regions where Russian was still an official language, such as Kazakhstan. Towards the last years of perestroika and the beginning of independence, emigration of ethnic Russians to Russia increased rapidly. According to some sources, the number Russians living in Kazakhstan decreased from 6 million to 4.5 between 1989 and 1990. For the same period, the Kyrgyzstan lost around $34 \%$ of its ethnic Russian population. In Turkmenistan, where the percentage of Russians had reached 9.4 of the entire population in 1989, there are currently two percent of Russians residing (Peyrouse, 2007: 246-247). Additional incentives for Russian outmigration were the economic crisis in the USSR that resulted in wage differences between Russia and Central Asia as well as ethnic clashes that broke out in the region, which in turn bore discriminatory attitudes towards Russians that had until then been treated with privilege.

Towards the last years of the perestroika period, the USSR's emigration procedures became less strict, its relations with the Western countries improved, and reunified Germany started welcoming ethnic Germans from the former Soviet Union. Ethnic Germans who had been resettled during the Stalinist period in the Central Asian region and had started emigrating in fairly small numbers already during the 1970s began leaving in masses in 1990. The remaining Jews emigrated as well, switching their destination from Israel to the USA, then in addition to Western Europe, making Germany their primary destination in the 1990 s.

\section{MIGRATION PATTERNS IN CENTRAL ASIA AND RUSSIA IN THE 1990'S: THE FALL OF THE SOVIET UNION AND FURTHER INCREASE OF EMIGRATION AND MOBILITY RATE}

Between 1990 and 1999, Germany received 1.63 million ethnic Germans from the former Soviet Union in the framework of the German repatriation program (Spätaussiedler). Approximately 120,000 Jews emigrated from the ex-Soviet countries to Germany as well (Dietz, 2000: 1). The majority of these emigrants belonged to families who had been resettled forcefully during the Stalinist period to the Central Asian region, mainly to Kazakhstan. According to the data provided by the Statistical Committee of the 
Commonwealth of Independent States, 49,505 ethnic Germans left Kazakhstan in 1997 (IOM 1999: 76). From Kyrgyzstan, 2,183 ethnic Germans emigrated to their historical homeland within the same year (IOM, 1999: 91). The statistical information indicates that the number of ethnic Germans tended to be much smaller for other Central Asian countries for 1997. Uzbekistan, for instance, recorded the emigration of 917 ethnic Germans, Turkmenistan recorded 345, and Tajikistan, only 247. The reason for this stark difference is not only the large proportion of forced settlement of ethnic Germans primarily in Kazakhstan, but also the fact that many ethnic Germans emigrated already in the earliest years of the independence of their countries of residence and at a time when Germany did not yet require language proficiency for entry into Germany, i.e. prior to 1996. This is particularly true for Tajikistan where the outbreak of ethnic clashes and the Civil War in 1992 resulted in massive emigration and a sharp decline in the number of ethnic Germans. Whilst in 1989 33,000 ethnic Germans were registered as residing in Tajikistan (IOM, 1999: 139), the number of ethnic Germans and Jews that left Tajikistan between 1990 and 1992 amounted to 230,000, taken together (Bushkov, 2000: 155).

The emigration of ethnic Germans was not the only migratory consequence of the Civil War in Tajikistan. The violent clashes of 1992-1997 led other ethnic minorities as well thousands of Tajikistanis to leave the country. During this period 697,653 Tajikistanis were registered as internally displaced, however the majority returned to their place of origin in 1997. Thousands fled to Afghanistan, Uzbekistan, Kazakhstan, Turkmenistan, and Russia. The Russian Federal Migration Service reported in 1998 that 50,192 refugees and 126,625 forced migrants were residing in the Russian Federation. The majority of those who had fled to countries other than Russia had repatriated to Tajikistan when the conflicting parties signed a peace treaty in 1997. Those Tajiks who had been granted refugee status in Russia stayed permanently and have meanwhile obtained Russian citizenship. In 1997-1998, together with half of the 60,000 Tajiks who had fled to Afghanistan from the Civil War, Tajikistan received approximately 2,500 Afghans who had fled from the armed conflicts in Afghanistan. These people have meanwhile obtained refugee status in Tajikistan (IOM, 1999: 139-140).

The Russian-speaking population continued emigrating from all the Central Asian republics. Kazakhstan, while experiencing a major outflow of population, allowed all persons with Kazakh ancestry residing outside the borders of the republic to return to their historic homeland. By the mid-1990s, notwithstanding the repatriation of approximately 160,000 ethnic Kazakhs from the neighboring countries and due to large-scale emigration of ethnic minorities, the population of this country dropped from 17 million to 15 million. In 1998, the Government of Kazakhstan implemented a state-sponsored repatriation program in order to alleviate the demographic crisis. The program was conceptualized to facilitate the settlement of repatriates (oralman) by offering assistance to employment, access to a number of social benefits, and support of repatriates through integration programs (Darieva, 2005).

Similar to Kazakhstan, albeit on a much smaller scale, Kyrgyzstan and Turkmenistan also allowed ethnic Kyrgyz and Turkmen migrants to return to their respective historic homelands from neighboring countries, although the governments have not yet opted to provide the returnees with financial means. The International Organization 
for Migration is currently working together with NGOs and the Kyrgyz government to promote the development of a legal framework for a return project of ethnic Kyrgyz called kairylman (returnee).

Poor ecological conditions around the evaporating Aral Sea (Karakalpak Autonomous Province and Xorazm Region in Uzbekistan) forced thousands move to different areas. By 1997, the number of ecological migrants was reported to have reached 16,000 . Besides the economic crisis affecting all the republics of the former Soviet Union with the transition to the market economy, the governments of the Central Asian republics were confronted with challenges regarding domestic and foreign policies. Managing the migration of the population was hence not at the top of their agenda. Although the newly independent countries adopted their own citizenship policies and introduced citizenship passports that enabled their residents to travel rather freely, challenges such as irregular migration, illegal border-crossing, and trafficking in human beings did not gain much attention. Only towards the end of the 1990s and the beginning of the $21^{\text {st }}$ century did the governments start making efforts to solve these problems. ${ }^{20}$ Apart from Kazakhstan and Turkmenistan, three countries of the region, viz. Kyrgyzstan, Tajikistan, and Uzbekistan, have witnessed a large outflow of irregular labor migrants to Russia since the late 1990s and early 2000. According to official sources, there are approximately 5-7 million labor migrants in the Russian Federation and half of them are estimated to be from Central Asian countries (IOM, 2008).

\section{CONCLUSION}

We have seen in this article that labor migration from Central Asia to Russia as it exists today has been structured by a complex array of political, economic and social factors. Through the historical trajectory of migrations from the earliest days of the Soviet Union until its demise in 1991, it has been illustrated that the population of the Soviet Union not only had strict constraints to movement but also was subject to forced relocations between different regions. Nonetheless, Soviet citizens increasingly engaged themselves in rural-to-urban migrations which expanded the urban population to a considerable extent. As far as Central Asia is concerned, it has been shown that the region continuously experienced as much in-migration as out-migration of people.

It has been illustrated in this article that labor migrants from Central Asia to Russia started to arrive already during the Soviet period. The current flow of labor force is therefore not an entirely new phenomenon. The party-state managed labor migration of Central Asia's workable population to Russia in the late 1970s and early 1980s was to enhance production through the cultivation of virgin lands. Together with Central Asian recruitees in the Soviet Army in Russia and Central Asian students at Russian higher education institutions, this generation of pioneer labor migrants may have played a significant role in the formation of migrant networks.

20 See IOM reports between 1999 and 2002.

REMI 2010 (26) 3 pp. 9-30 
In studying the current patterns of migration and, in particular, increased labor migration, we should bear in mind the ethnic heterogeneity of the population in this region. The three countries of origin (Kyrgyzstan, Tajikistan, and Uzbekistan) share a common Soviet past: it started with the annexation of their historical territories to the Russian Empire, the process of administrative territorial border demarcation in the 1920s and 1930s and the creation of nationalities by the Soviet state, and it continued as the states underwent the Sovietization processes, including the ideological project of 'friendship and brotherhood of nations' that gradually molded the society in the region.

\section{References}

ABAZOV Rafiz (2000) Migration of Population, the Labor Market and Economic Changes in Kirghizstan, in Hisao Komatsu, Chika Obiya and John S. Schoeberlein Eds., Migration in Central Asia: Its History and Current Problems, JCAS Symposium Series 9, Osaka, Japan Center for Area Studies, National Museum of Ethnology, pp. 209-235.

BERDINSKIKH Viktor (2005) Spetsposelentsy. Politicheskaia ssylka narodov sovetskoi Rossii [Special settlers. Political Exile of the Peoples of the Soviet Russia], Moscou, Novoe Literaturnoe Obozrenie, $527 \mathrm{p}$.

BERTHOMIĖRE William (2003) Immigration from the Former Soviet Union. Measure of the Impacts on Jerusalem and on the Settlements of the West Bank and Gaza, The Arab World Geographer, 6 (2), pp. 249-264.

BUCKLEY Cynthia (1995) The Myth of Managed Migration. Migration Control and Market in the Soviet Period, Slavic Review, 54 (4), pp. 896-916.

BUCKLEY Cynthia, RUBLE Blair A. and HOFMANN Erin T. (Eds.) (2008), Migration, Homeland and Belonging in Eurasia, Baltimore, Johns Hopkins University Press, 362 p.

BUGAI N.-F. and KOTSONIS A.-N. (1999) “Obiazat' NKVD SSSR ...vyselit' grekov”, "Assign the NKVD of the USSR to relocate the Greeks", Moscou, Insan, 159 p.

BUSHKOV Valentin I. (2000) Population Migration in Tajikistan: Past and Present, in Hisao Komatsu et al. Eds., Migration in Central Asia: Its History and Current Problems, JCAS Symposium Series 9, Osaka, Japan Center for Area Studies, National Museum of Ethnology.

BUWALDA Petrus (1997) They Did not Dwell Alone. Jewish Emigration from the Soviet Union 1967-1990. Washington DC, The Woodrow Wilson Center Press, Baltimore and London, The Johns Hopkins University Press, 297 p.

COLLINS Kathleen (2006) Clan Politics and Regime Change in Central Asia, Cambridge, New York, Cambridge University Press.

DARIEVA Tsypylma (2005) Recruiting for the Nation: Post-Soviet Transnational Migrants in Germany and Kazakhstan, Siberian Studies, http://www.siberianstudies.org/publications/PDF/ ridarieva.pdf.

DIETZ Barbara (2000), German and Jewish migration from the former Soviet Union to Germany: background, trends and implications, Journal of Ethnic and Migration Studies, 26 (4), pp. 635-652.

EDGAR Adrienne L. (2004) Tribal Nation: The Making of Soviet Turkmenistan, Princeton, Princeton University Press, $296 \mathrm{p}$.

GARCELON Marc (2001) Colonizing the Subject: The Genealogy and Legacy of the Soviet Internal Passport, in Jane Caplan and John Torpey Eds., Documenting Individual Identity. The Development of State Practices in the Modern World, Princeton and Oxford, Princeton University Press, pp. 83-100. 
GIBSON James R. (1991) Interregional Migration in the USSR, 1981-1985 and 1971-1975, The Canadian Geographer/Le Géographe canadien, 35 (2), pp. 142-156.

HIRSCH Francine (2005) Empire of the Nations. Ethnographic Knowledge and the Making of the Soviet Union, Ithaca and London, Cornell University Press, 367 p.

IOM INTERNATIONAL ORGANIZATION FOR MIGRATION (1999) Migration in the CIS 19971998, IOM Publications, $215 \mathrm{p}$.

IOM INTERNATIONAL ORGANIZATION FOR MIGRATION (2008) Migration in the Russian Federation. A Country Profile 2008, IOM Publications, 104 p.

ISLAMOV Bakhtior (2000) Migration of Population in Independent States of Central Asia, in Hisao Komatsu, Chika Obiya and John S. Schoeberlein Eds., Migration in Central Asia: Its History and Current Problems, JCAS Symposium Series 9, Osaka, Japan Center for Area Studies, National Museum of Ethnology, pp. 179-197.

KOCAOĞLU Timur (2000) Turkistan Abroad: The Political Migration - From the Soviet and Chinese Central Asia (1918-1997), in Hisao Komatsu, Chika Obiya and John S. Schoeberlein Eds., Migration in Central Asia: Its History and Current Problems, JCAS Symposium Series 9, Osaka, Japan Center for Area Studies, National Museum of Ethnology, pp. 113-126.

KOROBKOV Andrei V. (2008) Post-Soviet Migration: New Trends at the Beginning of the TwenyFirst Century, in Cynthia J. Buckley, Blair A. Ruble and Erin T. Hofmann Eds., Migration, Homeland and Belonging in Eurasia, Baltimore, Johns Hopkins University Press, pp. 69-98.

KOMATSU Hisao, Chika OBIYA and John S. SCHOEBERLEIN (Eds.) (2000) Migration in Central Asia: Its History and Current Problems. JCAS Symposium Series 9, Osaka, Japan Center for Area Studies, National Museum of Ethnology, $245 \mathrm{p}$.

LEWIS Robert A. and Richard ROWLAND (1979) Population redistribution in the USSR: its impact on society, 1897-1977, New York, Praeger, 485 p.

LUBIN Nancy (1984) Labor and Nationality in Soviet Central Asia. An Uneasy Compromise, Hong Kong, MacMillan Press, 305 p.

MATTHEWS Mervyn (1993) The Passport Society. Controlling Movement in Russia and the USSR, Boulder, San Francisco, Oxford, Westview Press, 118 p.

MEGORAN Nick (2002) The borders of eternal friendship? The politics and pain of nationalism and identity along the Uzbekistan-Kyrgyzstan Ferghana Valley boundary, 1999-2000, PhD Dissertation defended at Sydney Sussex College, Cambridge, UK, http://www.staff.ncl.ac.uk/ nick.megoran/pdf/nick_megoran_phd.pdf.

PEYROUSE Sébastien (2007) Rückkehr und Aufbruch. Zentralasiatische Migrationsströme, in Machtmosaik Zentralasien. Traditionen, Restriktionen, Aspirationen, Osteuropa, 57 Jahrgang, 8, Stuttgart, Deutsche Verlags-Anstalt, pp. 245-255.

POLYAN Pavel (2001) Ne po svoei vole. Istoriia i geografiia prinuditel'nykh migraciy v SSSR [Not by their own will. History and geography of forced migrations in the USSR]: Publication of the Human Rights Organization Memorial.

ROMAN Meredith (2002) Making Caucasians Black: Moscow Since the Fall of Communism and the Racialization of Non-Russians. Journal of Communism Studies and Transition Politics, 18 (2), pp. 1-17.

ROY Olivier (2000) The New Central Asia: Geopolitics and the Birth of Nations, New York, I.B. Tauris, $240 \mathrm{p}$.

RUBIN Barnett R. and Nancy LUBIN (Eds.) (1999) Calming the Ferghana Valley. Development and Dialogue in the Heart of Central Asia, Report of the Ferghana Valley Working Group of the Center for Preventive Action, New York, The Century Foundation Press, 196 p.

RYVKINA Rozalina V. (1996) Yevrei v postsovetskoi Rossii - Kto oni? Sotsiologicheskii analiz problem rossiiskogo evereistva [Jews in Post-Soviet Russia - Who are They? Sociological Analysis of Problems Related to Russian Jewishness] (In Russian.) Moskva, URSS.

SAHADEO Jeff (2007) 'Druzhba Narodov' or second class citizenship? Soviet Asian migrants in a post-colonial world, Central Asian Survey, 26 (4), pp. 559-579. 
SHIGABDINOV Rinat and NIKITENKO Georgii (2000) Migration Processes in the West of Central Asia in the Late Nineteenth and the Twentieth Centuries, in Hisao Komatsu, Chika Obiya and John S. Schoeberlein Eds., Migration in Central Asia: Its History and Current Problems, JCAS Symposium Series 9, Osaka, Japan Center for Area Studies, National Museum of Ethnology, pp. 87-111.

SMITH Graham (1996) The Nationalities Question in the Post-Soviet States, $2^{\text {nd }}$ Edition. London and New York, Longman, $389 \mathrm{p}$.

WIMBUSH S. Enders and PONOMAREFF Dmitry (1979) Alternatives for Mobilizing Soviet Central Asian Labor: Outmigration and Regional Development, A Project AIR FORCE report prepared for the United States Air Force, Santa Monica, CA, Rand Corporation, 38 p.

ZASLAVSKY Viktor and BRYM Robert J. (1983) Soviet-Jewish Emigration and Soviet Nationality Policy, Hong Kong, The Macmillan Press, 185 p.

ZAIONCHKOVSKAYA Zhanna A. (1999) Vnutrenniaia migraciia $v$ Rossii $i v$ SSSR $v$ XX veke kak otrajenie social'noi modernizacii [Internal Migration in Russia and the USSR in the $20^{\text {th }}$ century as the Reflection of Social Modernization], in Mir Rossii, 4, pp. 22-34, http://www.ecsocman. edu.ru/data/546/989/1219/1999_n4_p22-34.pdf (05/12/2010). 


\section{La mobilité des migrants d'Ouzbékistan : transport, frontières et circulation migratoire}

\section{Julien THOREZ ${ }^{1}$}

Dlusieurs vagues migratoires se sont succédé depuis la seconde moitié des années 1980 pour faire de l'Ouzbékistan un pays d'émigration². La fin de la période soviétique a vu l'intensification et l'internationalisation des flux migratoires parmi les populations " russophones $»^{3}$, suite à la décision des autorités soviétiques d'assouplir les conditions d'émigration en 1987 (de Tinguy, 2004 ; Korobkov et Zaïonchovskaia, 2004) ${ }^{4}$. Commun à tous les pays d'Asie centrale et du Caucase, ce processus a pris en Ouzbékistan une résonance particulière après les violences interethniques dont furent victimes les Meskhètes dans le Ferghana en 1989. Plusieurs centaines de milliers de Russes, de Tatars, d'Ukrainiens, d'Allemands, de Juifs ou de Grecs ont depuis définitivement quitté l'Ouzbékistan indépendant tandis que les populations " centre-asiatiques » (Ouzbeks, Tadjiks, Karakalpaks) demeuraient à l'écart de ce flux qui a fondamentalement transformé la société ouzbékistanaise (Maksakova, 2009).

1 Géographe, Chargé de recherche au CNRS, Mondes iranien et indien, 27, rue Paul Bert, 94204 Ivry-sur-Seine ; julienthorez@hotmail.com

2 Cet article a été rédigé dans le cadre du programme « Le Caucase et l'Asie centrale : un autre sud?», financé par l'ANR (2008-2012).

3 Cette catégorie désigne les populations arrivées en Asie centrale de façon volontaire, encadrée ou forcée, durant les périodes tsaristes et soviétiques (Laitin, 1998 ; Thorez, 2009).

4 Les flux d'immigration se sont presque taris durant les années 1990 d'autant que, contrairement au Kazakhstan et au Kirghizstan, l'Ouzbékistan n'a pas mis en œuvre de politique de « rapatriement » des minorités co-ethniques de l'étranger, pourtant nombreuses au Tadjikistan, au Kirghizstan, au Kazakhstan, au Turkménistan ou en Afghanistan (Ferrando, 2010).

5 Héritage des politiques tsariste et soviétique, une distinction est toujours opérée en Ouzbékistan entre la citoyenneté - ouzbékistanaise - qui renvoie à une identité civique, et la nationalité ouzbèke, tadjike, russe, etc. - qui correspond à une identité ethnique. 
Depuis la fin des années 1990, le développement des migrations de travail, dont les populations centre-asiatiques sont les principaux acteurs, produit une seconde vague d'émigration. L'émergence de ces flux saisonniers et temporaires s'est déroulée dans un contexte de crise de la mobilité et du système de transport - proche d'un million de passagers en 1990, le trafic de l'aéroport de Samarkand a été inférieur à 100000 passagers en 1995 (Thorez, 2005) - qui invite à questionner les fondements de la dynamique migratoire ainsi que les modalités de circulation des travailleurs-migrants. D'autant que cette mise en mouvement contraste avec l'atonie migratoire qui caractérisait les populations des qishloq (village) et des mahalla (communauté territorialisée correspondant à un quartier, conjuguant liens de voisinage et de parenté) durant la période soviétique. La généralisation des migrations de main-d'œuvre façonne de nouvelles territorialités qui participent à la redéfinition du positionnement de l'Ouzbékistan dans l'espace mondialisé. Étendu de l'Asie de l'Est à l'Amérique du Nord, mais polarisé par la Russie, l'espace migratoire ouzbékistanais articule des dynamiques globales et des dynamiques postcoloniales, qui illustrent la complexité de la transformation post-soviétique.

\section{MIGRATIONS DE TRAVAIL : L'ÉMERGENCE DE NOUVELLES TERRITORIALITÉS}

\section{Un flux difficilement quantifiable}

Les migrations de travail ont pris de l'importance depuis le tournant des années 2000. Toutefois, aucune source ne donne d'informations permettant de définir avec précision l'ampleur de ce processus. Les données communiquées par les institutions de l'État ouzbékistanais $^{6}$ tendent à minimiser un phénomène qui peut être interprété comme une manifestation de la dégradation de la situation économique et sociale survenue au cours de la période contemporaine (Laruelle, 2010a) ${ }^{7}$. Dans les pays de destination des travailleurs ouzbékistanais, les administrations ne recensent officiellement que les migrants disposant d'un permis de résidence ou de travail. Experts, chercheurs, associations et organisations non gouvernementales proposent des estimations du nombre de migrants plus élevées que les statistiques officielles : elles oscillent entre 2 et 8 millions de migrants. Ces évaluations témoignent de l'investissement de la société dans le processus d'externalisation de la main-d'œuvre et d'extraversion de l'accumulation du capital. Mais l'analyse des équilibres démographiques invite à considérer qu'environ 2 à 3 millions d'Ouzbékistanais travaillent saisonnièrement ou temporairement à l'étranger. Quoi qu'il en soit, l'Ouzbékistan est devenu le premier pays d'origine des Gastarbeiter employés en Russie, en fournissant environ $25 \%$ de la main-d'œuvre immigrée en 2008, de même qu'au Kazakhstan (Zajončkovskaâ, Mkrčân et Tûrûkanova, 2009 ; Sadovskaâ, 2009).

6 D'après l'Agence pour les migrations internationales de main-d'œuvre, créée en 2001 au sein du ministère du Travail et de la Sécurité sociale, seulement 262900 Ouzbékistanais travaillaient à l'étranger en 2005 (Maksakova, 2009).

7 Massot estime même que «migrer peut être considéré comme une prise de position politique, un acte militant » (2009). 


\section{Migrer, une pratique post-soviétique?}

\section{Une activité migratoire réduite}

L'ampleur des migrations contemporaines contraste avec l'enracinement des populations centre-asiatiques (Ouzbeks, Tadjiks, etc.) à la fin de la période soviétique. Au recensement de 1979, les Ouzbékistanais détenaient les taux de mobilité résidentielle les plus faibles de l'URSS (Maksakova, 1986) ${ }^{8}$. Aussi le peuplement ouzbek était-il caractérisé par un double ancrage, national et rural, quelquefois analysé comme l'expression d'une surpopulation corrélée à des formes de sous-développement (Patnaik, 1995). Pourtant, les autorités avaient tenté de stimuler les migrations à destination des régions soviétiques connaissant un déficit de main-d'œuvre (Sibérie, etc.) (Tarasova, 1985). À l'échelle de l'Ouzbékistan, elles avaient également agi sur la répartition de la population en organisant le peuplement de périmètres agricoles nouvellement mis en valeur dans la Steppe de la Faim et autour de Karchi, notamment depuis les oblast' du Ferghana (Maksakova, 2001). Cette politique volontariste visait à réduire les inégalités régionales du marché du travail et à contenir les tensions sociales dans les régions densément peuplées.

\section{L'atonie migratoire, une manifestation de sous-développement?}

Considérées dans le contexte ouzbékistanais des années 1970-1980 comme un levier de promotion sociale, les migrations avaient été auparavant contrôlées et limitées par l'État soviétique au moyen de dispositifs administratifs contraignants (permis de résidence [propiska], passeport intérieur, permis de travail, cf. Bater, 1989). Dissuasives, ces mesures communes à toutes les républiques soviétiques pouvaient apparaître comme une entrave à la mobilité, en particulier dans les campagnes. Toutefois, au moment où la population rurale s'enracinait en Ouzbékistan, plusieurs régions soviétiques connaissaient un intense exode rural et un processus de dépeuplement. En Russie, la population rurale s'est ainsi contractée de 72 à 39 millions d'habitants entre les recensements de 1939 et de 1989.

En Ouzbékistan, les réticences des populations centre-asiatiques à migrer dépendaient moins de la législation que de facteurs économiques, sociaux et culturels. La faible mobilité résidentielle s'inscrivait dans un contexte spécifique aux régions méridionales de l'Asie centrale marqué par l'organisation autocentrée des sociétés urbaines et rurales. D'une part, les familles turkestanaises, fondées sur une résidence patrilocale, exerçaient une attraction sur l'ensemble de leurs membres, d'autant plus forte que, dans les milieux ruraux, le niveau de vie était corrélé à la taille des familles (Zûzin, 1983 ; Mullâdžanov et Ubajdullaeva, 1988). D'autre part, l'inscription des individus dans le mahalla a longtemps favorisé l'enracinement des populations. Pour les populations centre-asiatiques, migrer impliquait en outre de s'éloigner voire de se couper de réseaux sociaux et familiaux dont le rôle était fondamental dans l'orientation des trajectoires individuelles et dans l'accès aux biens et aux services. De surcroît, quitter le village et/ou l'Ouzbékistan supposait de se confronter à un environnement russophone, souvent perçu comme étranger voire

$877 \%$ des habitants de la RSS d'Ouzbékistan ( $88 \%$ des ruraux) résidaient dans le même lieu depuis leur naissance. 
comme contradictoire avec les traditions nationales et religieuses associées aux mahalla et aux qishloq. Des frontières symboliques s'ajoutaient donc aux dispositifs législatifs pour limiter la mobilité résidentielle des Ouzbeks et des Tadjiks.

La spécialisation agricole des campagnes participait également de la faible demande migratoire. La production de coton, qui a connu un essor considérable dans le cadre du système soviétique de production et d'échanges, employait en effet une maind'œuvre abondante. Malgré l'importance du travail manuel, les campagnes connaissaient pourtant un sous-emploi chronique, partiellement compensé par l'emploi du surplus de main-d'œuvre dans les exploitations auxiliaires domestiques, i.e. dans l'agriculture privée (Patnaik, 1995). Cette situation aurait pu alimenter les flux d'émigration, mais les agriculteurs, dans l'ensemble mal formés, peinaient à se réorienter vers des métiers industriels ou tertiaires. De plus, ils devaient faire face à la concurrence d'une main-d'œuvre russophone qualifiée, dont la présence dans les villes a été analysée comme un obstacle aux migrations des campagnes vers les villes (Topilin, 1975).

Si les facteurs sociaux et culturels sont parfois présentés comme le fondement de l'atonie migratoire des Ouzbeks et des Tadjiks (Patnaik, 1995), les populations centreasiatiques semblaient également jouir d'un niveau de vie peu propice à la mobilité résidentielle. Dans les années 1980, les kolkhoziens et les sovkhoziens percevaient des rémunérations inférieures à la moyenne soviétique, mais les familles rurales bénéficiaient de la production et des revenus tirés de la vente des excédents des exploitations auxiliaires domestiques. Or, ces dernières avaient officiellement fourni la moitié des légumes, des fruits et de la viande ainsi que les deux tiers du lait produits en 1990. Issus de la vente directe, des achats de l'État et de l'écoulement des produits frais sur les bazars 9 , les revenus de l'agriculture privée représentaient de ce fait davantage que les rémunérations reçues par les agriculteurs dans les exploitations collectives (Tašbaeva et Suvurov, 1989). Alors que le coût de la vie était en Ouzbékistan inférieur à la moyenne soviétique (Zûzin, 1983), les activités non contrôlées par l'État augmentaient considérablement les ressources familiales et contribuaient par conséquent à limiter l'attraction des villes et des autres régions soviétiques. Dès lors, les politiques d'incitation à la mobilité conduites à la fin de la période soviétique furent sans grand effet. Au cours des années 1970-1980, 1'activité migratoire des populations russophones, dont le solde s'inversait pour devenir négatif, contrastait avec l'ancrage des populations centre-asiatiques - en 1980, les Ouzbeks représentaient $70 \%$ de la population, mais seulement $30 \%$ des migrants (Maksakova, 1986).

\section{Stabilité résidentielle et migrations professionnelles}

La stabilité résidentielle des populations centre-asiatiques des villes et des campagnes d'Ouzbékistan coexistait cependant avec différentes formes de mobilité professionnelle. À partir des années 1960, les migrations pendulaires ont connu un développement régulier autour des principales agglomérations, stimulé par l'industrialisation et la modernisation des moyens de transport (Maksakova, 1986). Dans la majorité des cas, les entreprises organisaient le transport des salariés par autocar, entre les villages périurbains et les usines (Aliakberova et al., 1990).

9 En 1985, $50 \%$ des légumes, $40 \%$ des fruits et $25 \%$ de la viande consommés par les ouvriers et les salariés d'Ouzbékistan ont été achetés sur les marchés paysans (Ivatov, 1994).

REMI 2010 (26) 3 pp. 31-57 
Parallèlement aux migrations pendulaires, des migrations saisonnières mobilisaient des travailleurs ouzbékistanais. En Ouzbékistan, des paysans se rendaient dans les régions méridionales (Surkhan-Daria, Kachka-Daria) pour participer à la culture du riz et de l'oignon (Maksakova, 2001). En septembre-octobre, plusieurs milliers de jeunes urbains, étudiants ou travailleurs mis à disposition par leurs autorités respectives, étaient affectés à la cueillette du coton. À l'échelle soviétique, les migrants saisonniers, en grande majorité des hommes travaillant en été comme ouvriers qualifiés sur des chantiers de construction, se dirigeaient vers la Russie et secondairement vers le Kazakhstan (Aliakberova et al., 1990 ; Maksakova, 2001). À côté de ces migrations planifiées, il existait également des formes de mobilité non encadrée. Durant l'été, des Ouzbékistanais partaient pour la Russie afin de vendre sur les marchés urbains les fruits et les légumes produits dans les oasis (Reeves, 2010). Organisés ou informels, ces différents flux s'inscrivaient dans un système de mobilité plus large, qui incluait notamment d'autres déplacements professionnels et des mobilités touristiques. En ce sens, l'ancrage résidentiel exceptionnel des Ouzbeks ne signifiait pas que la société était immobile, repliée ou isolée. Si la mobilité des Centreasiatiques était moindre que celle des populations exogènes, leur horizon géographique ne se bornait pas aux limites des qishloq et des mahalla ni aux frontières de l'Ouzbékistan. Toutefois, parmi les populations ouzbèkes, la mobilité des hommes différait de celle des femmes, les itinéraires de vie des premiers - soviétiques - contrastant avec les territorialités centre-asiatiques des secondes.

\section{Les déterminants de la mobilité : l'essor des migrations de travail}

La disparition de l'URSS, quelquefois présentée comme une date charnière dans l'histoire des migrations ouzbékistanaises (Massot, 2009), n'a pas coïncidé avec une modification immédiate des pratiques migratoires des Ouzbeks. Apparues à la fin des années 1990 et intenses depuis 2002-2003, les migrations de travail ne se sont développées en Ouzbékistan qu'après leur essor au Tadjikistan et au Kirghizstan (Brusina, 1998). Une approche économique des migrations indiquerait que la politique de réformes graduelles menée par l'Ouzbékistan a permis d'amortir la crise post-soviétique, en préservant quelque peu les salaires et les emplois, y compris dans les campagnes où, dans un premier temps, ont été maintenues des exploitations collectives (Abdullaev, 2008). La faiblesse du flux d'émigration aurait aussi été liée au développement de l'économie de marché et à l'apparition de nouvelles activités productives et commerciales, quelquefois informelles, qui ont fourni du travail et assuré un revenu à une part importante de la population, notamment sur les bazars. Au cours des années 1990, la situation économique dégradée limitait enfin les besoins en main-d'œuvre de la Russie et du Kazakhstan, qui connaissaient pourtant une crise démographique.

\section{Pression démographique et mutations économiques : au cœur de la demande migratoire}

Si l'essor des migrations de travail fut postérieur à la crise post-soviétique, l'émergence des nouvelles pratiques migratoires renvoie fondamentalement à la situation économique et sociale, en dépit de la complexité des mécanismes qui structurent la demande de mobilité. En Ouzbékistan, l'extraversion de la main-d'œuvre traduit avant tout la mise 
en œuvre de stratégies individuelles et familiales destinées à s'affranchir des difficultés économiques. Malgré la croissance enregistrée depuis la seconde moitié des années 1990, les revenus restent modestes, notamment dans les campagnes ${ }^{10}$. Phénomène classique, les plus pauvres ne peuvent mobiliser les ressources nécessaires pour migrer, mais la majorité de la population vit dans un entre-deux économique insatisfaisant, mais suffisant pour financer des projets migratoires, alors même que le marché du travail connaît des tensions liées à la rapide croissance démographique (Tableau 1). Les années 2000 correspondent en effet à l'entrée sur le marché du travail des générations nombreuses nées au cours du pic de natalité des années 1980 . De ce fait, malgré la croissance économique, la création de nouveaux postes dans les secteurs public et privé ne répond que partiellement à l'augmentation de la population active ${ }^{11}$. Les migrations de travail contribuent alors à pallier l'insuffisance des revenus et à atténuer la pénurie d'emplois. Pour l'État, cette mobilité internationale saisonnière et temporaire permet de réguler un marché du travail, dont elle est désormais un segment essentiel (Maksakova, 2009).

Tableau 1 : La croissance démographique de l'Ouzbékistan (1991-2006)

\begin{tabular}{|l|c|c|c|}
\hline & 1991 & 2000 & 2006 \\
\hline Population totale (M) & 20,857 & 24,650 & 26,488 \\
\hline $\begin{array}{l}\text { Population en âge de } \\
\text { travailler (M) }\end{array}$ & 10,213 & 12,469 & 14,816 \\
\hline Population active (M) & 8,383 & 9,018 & 10,492 \\
\hline
\end{tabular}

Source : Comité des statistiques de l'Ouzbékistan

Dans les campagnes, l'accroissement des migrations de main-d'œuvre coïncide également avec l'accélération de la réforme agraire. Le démantèlement des exploitations collectives au profit d'une agriculture paysanne a participé à la mise en migration de la société rurale. En rupture avec les pratiques territoriales traditionnelles, plusieurs centaines de milliers de ruraux ont connu leur première expérience migratoire depuis le début des années 2000. Qu'elles soient internationales (saisonnières ou temporaires) ou nationales (pendulaires ou saisonnières), ces migrations concourent à la "prolétarisation » des campagnes - toujours très agricoles - de l'Ouzbékistan (Bazin, 2009). Dans le pays de villages qu'est l'Ouzbékistan, cette mobilité internationale s'apparente aussi à une forme classique d'externalisation de l'exode rural. Toutefois la généralisation des migrations depuis les campagnes vers l'étranger et vers les villes s'opère dans un contexte économique et technique spécifique où l'agriculture ne connait pas d'élévation du niveau de mécanisation ni de croissance significative de la production.

10 Le salaire mensuel « réel » serait de quarante à cinquante dollars.

11 La part des « ressources laborieuses » [trudovye resursy] s'est élevée de $49 \%$ en 1989, à $52 \%$ en 2000 puis à $59,5 \%$ de la population ouzbékistanaise en 2008 . 


\section{De la circulation commerciale à la mobilité migratoire : exploiter les diffé- rentiels frontaliers}

L'internationalisation des migrations de main-d'œuvre a aussi succédé à la redéfinition des conditions de résidence et de travail impulsée par l'État, au tournant des années 2000. À la suite d'attentats perpétrés à Tachkent en février 1999 et attribués par le pouvoir à des mouvements islamistes radicaux, le gouvernement a pris la décision de renforcer les mesures de sécurité et d'exercer des contrôles accrus sur la population (Trudovye..., 2005). Alors que le système du permis de résidence existe toujours, ces mesures ont surtout affecté les habitants permanents ou temporaires de Tachkent n'ayant pas d'enregistrement dans la capitale, c'est-à-dire les migrants. Parmi ces derniers, les hommes, d'une part, et les migrants originaires du Ferghana, région dont les principaux dirigeants du Mouvement Islamique d'Ouzbékistan étaient issus, d'autre part, furent particulièrement ciblés. Cette politique sécuritaire a contraint les migrants à modifier leurs pratiques territoriales. En premier lieu, elle a favorisé la féminisation des migrations intérieures, les femmes étant moins soumises à la pression policière (Artykova, 2007). En second lieu, elle a incité des migrants du Ferghana à se réorienter vers la Russie et le Kazakhstan où, après une décennie de crise, la demande de main-d'œuvre a fortement augmenté en raison de la croissance économique.

La projection vers l'étranger des travailleurs ouzbékistanais a en outre répondu à la législation adoptée en 2002 pour réguler les activités commerciales. Engagé dans une politique de substitution aux importations, le pays a établi des taxes à l'importation de $50 \%$ sur les produits alimentaires et de $90 \%$ sur les produits manufacturés et a limité les droits d'importation des commerçants-navetteurs, pour remédier au déséquilibre de la balance des paiements et stimuler la production nationale (Raballand, 2005). La nouvelle législation a également renforcé les contrôles sur l'origine et la qualité des produits et introduit de nouvelles démarches administratives. Ces dispositions, qui ont suscité des réactions hostiles sur les bazars, ont entravé l'activité des entreprises et des réseaux marchands, formels ou informels (Thorez, 2008). Tandis que dans les années 1990, ce secteur avait connu une forte croissance et créé de nombreux postes de travail, les mesures protectionnistes ont accentué la pression sur le marché du travail et accru le nombre de migrants potentiels. À certains égards, elles ont même été l'élément déclencheur de la massification des flux d'émigration. Les anciens commerçants-navetteurs (čelnoki) ont en effet utilisé leur expérience de la mobilité internationale pour travailler à l'étranger ou pour organiser les migrations, en recrutant et en plaçant auprès d'employeurs kazakhstanais et russes des candidats à l'émigration. Les travailleurs migrants ont aussi pu bénéficier de l'appui des entrepreneurs qui avaient délocalisé leur activité au Kazakhstan et en Russie pour se protéger des nouveaux dispositifs ouzbékistanais (Sadovskaâ, 2009). Malgré la nature différente de leur mobilité, les commerçants et les migrants mettent à profit les différentiels frontaliers et les inégalités économiques qui structurent désormais l'espace post-soviétique : en 2008, le PIB par habitant était estimé à 1030 dollars en Ouzbékistan, 8500 dollars au Kazakhstan et 11800 dollars en Russie. Ces écarts contribuent à stimuler des mobilités qui, à l'échelle de l'espace post-soviétique, aident à contenir les processus de désintégration territoriale symbolisés par les nouvelles frontières. 


\section{Migrer, un rite de transition au fondement de l'« ouzbékité »?}

Le cycle migratoire est quelquefois présenté comme un rite de transition au fondement de la construction collective de l'« ouzbékité », i.e. de l'identité de la nation ouzbèke indépendante (Massot, 2009). Expérience inédite de l'altérité, les migrations de travail renforceraient la cohésion d'une société dès lors consciente de ses « valeurs » et participeraient au changement social, à travers le statut de «nouvel Ouzbek » acquis par des migrants enrichis. Cette approche semble toutefois surestimer les effets politiques et identitaires des migrations de main-d'œuvre, alors que seuls quelques migrants travaillant en Europe de l'ouest ou en Amérique du nord ont pu réellement s'enrichir et s'élever dans la hiérarchie sociale, notamment dans les années 1990.

L'expérience migratoire apparaît en revanche essentielle dans la construction de la masculinité, en particulier dans les milieux où la proportion de femmes migrantes est faible, car migrer permet aux jeunes hommes d'affirmer leur statut social, en confirmant leur identité, en élargissant leurs compétences (Reeves, 2010). Les migrations de travail présentent ainsi des similitudes avec l'accomplissement du service militaire durant la période soviétique. Plus largement, la demande sociale stimule la mobilité des hommes, qui représentent 75 à $80 \%$ des migrants, car ces derniers migrent notamment pour se donner les moyens d'exercer leur statut de mari ou de père (Thorez, 2007a). En définitive, la société doit aujourd'hui composer avec des aspirations et des pratiques multiples, réguler les contradictions entre le départ en migration des hommes, qui déstabilise les familles, et la volonté de maintenir des structures sociales traditionnelles, en mobilisant notamment les revenus de l'émigration dans les pratiques matrimoniales.

\section{L'ESPACE MIGRATOIRE OUZBÉKISTANAIS}

\section{Flux postcoloniaux et flux globaux : les migrations internationales}

L'espace de circulation des migrants s'étend des pays du Golfe persique à l'Europe de l'ouest, de l'Amérique du nord à l'Asie orientale, région où la Corée du Sud est la principale destination des Ouzbékistanais. Mais $95 \%$ des migrants se déplacent au sein de l'ancien territoire soviétique, en direction de la Russie et du Kazakhstan (Figure 1). Révélatrice de l'hétérogénéité des projets migratoires, cette géographie de l'émigration traduit l'insertion des Ouzbékistanais dans les courants migratoires internationaux ainsi que l'ampleur des mobilités inscrites dans un contexte postcolonial : la Russie accueille entre $65 \%$ et $80 \%$ des migrants, le Kazakhstan entre $15 \%$ et $25 \%$ (Rynok..., 2007 ; Abdullaev, 2008).

\section{Un espace migratoire en expansion}

La diversification des destinations a naturellement entraîné un élargissement de l'espace migratoire, limité au territoire soviétique, sauf exception, jusqu'à la fin des années 1980. Cette extension s'opère dans un contexte de libéralisation de la mobilité quoique l'Ouzbékistan ait maintenu des dispositifs de contrôle de la circulation internatio- 


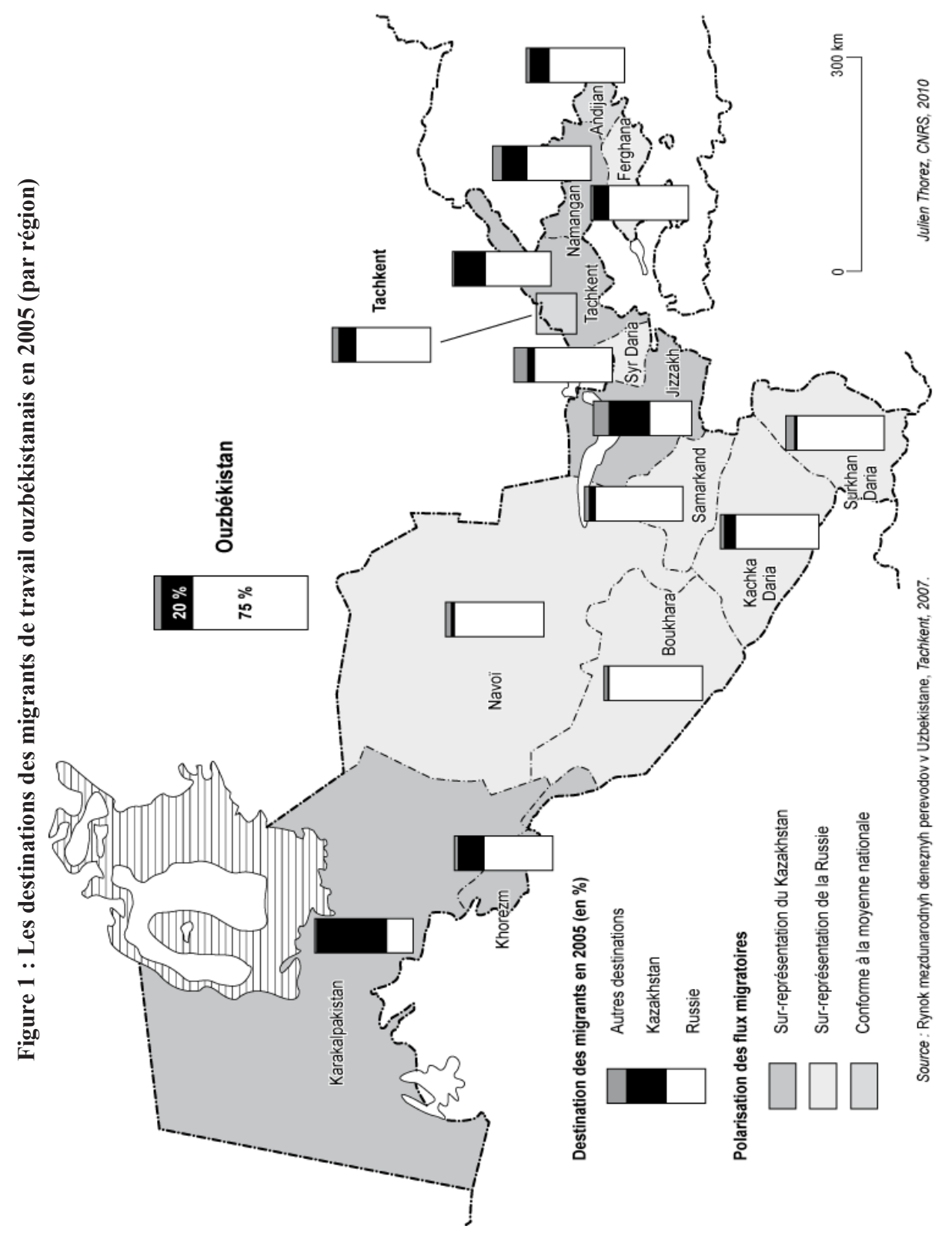


nale. La multiplication des flux d'émigration a aussi coïncidé avec l'internationalisation de la desserte de l'Ouzbékistan en particulier, le réseau des lignes aériennes internationales a connu une croissance spectaculaire depuis 1990, Tachkent étant désormais relié à plusieurs dizaines d'aéroports étrangers (Thorez, 2005). Cette évolution a toutefois moins répondu à la demande de mobilité des migrants qu'à la stratégie de développement d' $U z-$ bekistan Airways et à la demande politique de transport liée à l'affirmation de la souveraineté. À l'échelle internationale, seule la liaison Tachkent-Séoul semble connaître un essor corrélé à l'intensification des migrations de travail, plusieurs dizaines de milliers d'Ouzbékistanais travaillant en Corée du Sud.

Malgré l'internationalisation de l'offre de transport, l'« étranger lointain » reste peu accessible à la masse des travailleurs ouzbékistanais. Pourtant les destinations européennes et nord-américaines permettent aux migrants de prétendre à des revenus supérieurs aux sommes accumulées en Russie et au Kazakhstan ${ }^{12}$. Mais accéder à ces espaces implique de mobiliser des ressources économiques, culturelles et réticulaires inaccessibles à la majorité des migrants. Dans de nombreux cas, partir pour l' « étranger lointain » dépend de stratégies à long terme incluant l'apprentissage d'une langue étrangère et l'acquisition de compétences professionnelles (Belouin, 2010).

\section{Des flux polarisés par la Russie : une ancienne « métropole » accessible}

Malgré l'édification des frontières et la contraction de l'offre de transport dans les années 1990, la Russie conserve un niveau d'accessibilité élevé depuis l'Ouzbékistan, lié notamment à la proximité sociale et culturelle héritée du passé soviétique commun. Par ailleurs, conformément à l'accord signé en 1992 par les pays de la CEI qui affirme la libre circulation de leurs ressortissants, la législation russe garantit aux citoyens de l'Ouzbékistan le droit d'entrer sans visa sur le territoire national et, après enregistrement, d'y séjourner quatre-vingt-dix jours avec une autorisation temporaire de séjour, préalable à l'attribution d'une carte de séjour de trois ou cinq ans (Braux, 2009). En revanche, obtenir une autorisation de travail présente davantage de difficultés, en raison de la complexité des démarches administratives et de l'instauration d'un système de quotas ${ }^{13}$. Dès lors les migrants séjournent souvent légalement en Russie, mais y travaillent sans autorisation. Ce dispositif législatif, comme celui adopté par le Kazakhstan, amène de nombreux migrants à se rendre à l'étranger au terme d'un trimestre de présence en Russie. Ces déplacements vers le Kazakhstan ou l'Ukraine ont pour objectif de franchir la frontière et de retourner légalement en Russie. Il arrive aussi qu'une personne - elle-même migrante - se charge des passeports de plusieurs collègues et négocie à la frontière la régularisation des documents.

12 En moyenne, en 2007, les migrants dégageaient 100 dollars par mois en Russie, 300 dollars en Corée du Sud et 800 dollars aux États-Unis (Rynok..., 2007).

13 En 2007, après l'instauration d'un système de quotas, la Russie a établi une liste de secteurs d'activité, de métiers et de qualifications prioritaires : travaux de construction, de rénovation et de montage ; travailleurs agricoles qualifiés ; industries métallurgique et automobile ; chauffeurs et machinistes (Braux, 2009).

REMI 2010 (26) 3 pp. 31-57 


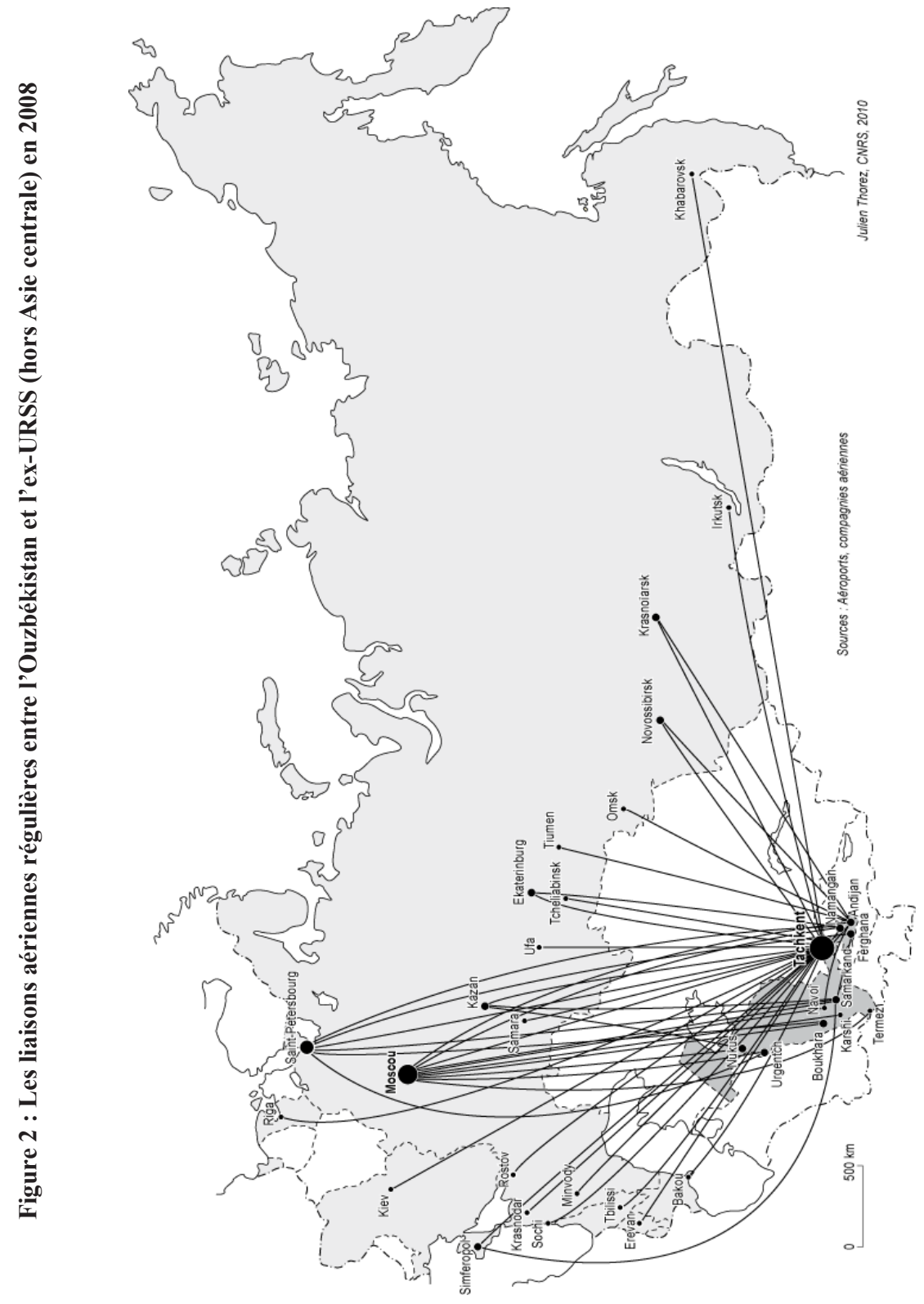


Un dense réseau de liaisons aériennes, ferroviaires et routières fait toujours de la Russie et de Moscou le pays et la ville les mieux desservis depuis l'Ouzbékistan (Figure 2). En 2009, trente-six lignes aériennes connectent onze villes ouzbékistanaises à dix-sept villes russes; les chemins de fer ouzbékistanais et russes exploitent huit liaisons au départ de Tachkent ; des centaines d'autocars et de minibus circulent entre les deux pays. Depuis le début des années 2000, l'offre de transport connaît une forte croissance soutenue à l'évidence par la mobilité des migrants, sans toutefois retrouver son niveau soviétique (Figures 2, 3 et 4). Elle se traduit par l'ouverture de nouvelles lignes aériennes et ferroviaires, notamment à destination de Saint-Pétersbourg, et par une augmentation de la fréquence de circulation des avions et des trains (Figures 2 et 3 ). En dépit du renforcement de l'offre de transport, des pénuries de billets peuvent survenir au printemps et en automne, périodes où la demande de mobilité est maximale, par exemple sur la liaison Tachkent-Moscou.

Les coûts de transport n'entravent pas la mobilité des travailleurs ouzbékistanais, malgré leur augmentation depuis 1990. La plupart des familles peuvent rassembler le capital suffisant pour financer le voyage d'un migrant d'autant que travailler en Russie permet généralement de rembourser rapidement les dettes contractées auprès de parents et/ou d'amis. En 2008, 200 euros suffisaient pour acheter un billet d'avion pour Ufa, Rostov, Tcheliabinsk ou Novossibirsk. Un billet de train en seconde classe coûtait entre Tachkent et Saratov 140000 sum (soit soixante-dix euros), entre Tachkent et Moscou 260000 sum (soit 130 euros) $)^{14}$. Le transport routier offre des tarifs moins coûteux, mais depuis l'Ouzbékistan, quatre à cinq jours sont nécessaires pour atteindre les régions centrales de la Russie. Ces possibilités de circuler aisément et légalement permettent à de nombreux travailleurs de se rendre en Russie au printemps et de rentrer en Ouzbékistan à l'automne. Ces mobilités saisonnières rythment désormais la vie des villes et des villages d'Ouzbékistan et l'on observe notamment le glissement vers l'hiver des cérémonies de mariage et de circoncision.

La localisation des villes desservies depuis l'Ouzbékistan témoigne de la dispersion des migrants sur l'ensemble du territoire russe (Figure 2). Toutefois, les régions centrales (Moscou et Saint-Pétersbourg) et les régions voisines de l'Asie centrale (ensemble VolgaOural, Sibérie occidentale) accueillent la majorité des migrants (Zajončkovskaâ, 2008) Plusieurs centaines de milliers d'Ouzbékistanais résident ainsi dans l'agglomération moscovite où, à l'instar des migrants tadjikistanais et kirghizstanais, ils travaillent dans les secteurs de la construction, de l'entretien, de la restauration, des services et du commerce, malgré l'entrée en vigueur en 2006 d'une loi interdisant aux étrangers d'exercer la profession de vendeur sur les marchés (Maksakova, 2009 ; Braux, 2009). Dans les régions méridionales, les migrants sont également embauchés dans l'agriculture, en particulier dans la production de fruits et légumes. La présence massive de la main-d'œuvre centre-asiatique en Russie, accompagne le développement économique, compense partiellement le

14 En 2000, selon les catégories de confort, le billet de train Tachkent-Moscou coûtait de 23840 à 35000 sum (de trente à quarante-cinq dollars) (Thorez, 2005).

15 En 2007, un quart des migrants ouzbékistanais travaillait à Moscou, près de $20 \%$ dans l'okrug fédéral de la Volga, près de $15 \%$ dans l'okrug fédéral de Sibérie, d'après le service fédéral des migrations.

REMI 2010 (26) 3 pp. 31-57 


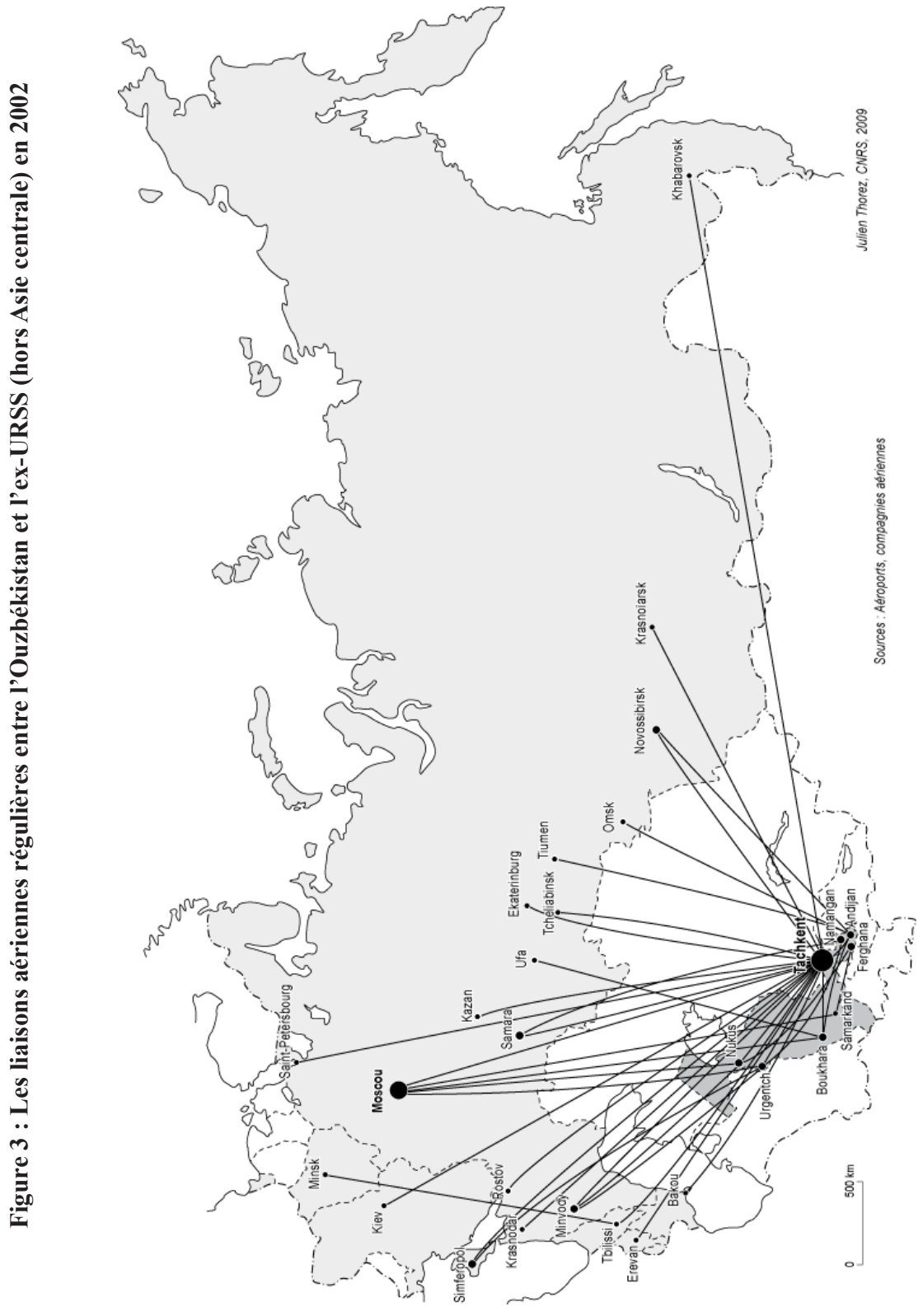


déficit démographique, mais ne va pas sans poser des questions sociales et politiques à une société affectée par des tendances xénophobes ${ }^{16}$. Dans ce contexte, la politique migratoire russe oscille entre des mesures de fermeté et des décisions inspirées par des positions plus libérales (Zajončkovskaâ, Mkrčân et Tûrûkanova, 2009).

\section{Flux locaux et flux régionaux : des migrations centre-asiatiques dirigées vers le Kazakhstan}

En Asie centrale, la mobilité des travailleurs ouzbékistanais se fait sous la forme de flux locaux dirigés vers les régions limitrophes du Kirghizstan et du Kazakhstan et de flux régionaux orientés vers les principaux pôles économiques du Kazakhstan : les régions pétrolières d'Aktau et d'Atyrau, la capitale économique, Almaty, et la capitale politique, Astana.

À l'échelle locale, les flux migratoires se sont développés dans les viloyat de Tachkent, de Syr Daria et de Djizakh en direction du Kazakhstan du Sud. Ces migrations intéressent surtout des ouvriers agricoles engagés pour la saison, le mois, la semaine ou la journée, à la production de fruits et de légumes ou à la cueillette du coton dans les districts de Sary Agach et de Makhtaaral. En 2001, bien que moins payés que la maind'œuvre locale, les ramasseurs de coton travaillant au Kazakhstan, qui viennent aussi des régions pauvres du Kachka Daria et du Surkhan Daria, pouvaient presque tripler leurs revenus (Thorez, 2005 ; Sadovskaâ, 2009). Embauchés également dans les secteurs de la construction et du commerce, les nombreux migrants participent activement au développement économique du Kazakhstan du Sud (Trudovye..., 2005 ; Sadovskaâ, 2009) ${ }^{17}$. Plus à l'ouest, le bassin caspien attire un quart des travailleurs ouzbékistanais, principalement originaires du Karakalpakistan et du Khorezm (Figure 1). Une minorité de migrants, souvent issus du Ferghana et de la région de Samarkand, se dirigent vers Almaty et Astana, où la construction, la restauration et le commerce sont leurs principaux domaines d'activité (Sadovskaâ, 2009).

La présence des travailleurs ouzbékistanais était également importante dans le Ferghana kirghizstanais, dans les régions d'Och et de Djalal-Abad, où leur séjour était de courte durée ${ }^{18}$. Originaires des régions de Namangan, de Ferghana et surtout d'Andijan, les migrants travaillaient dans l'agriculture ainsi que dans la construction, de nombreux travaux étant effectués par des brigades présentes au Kirghizstan le temps d'un chantier. Ce flux, qui chaque jour drainait 4000 à 5000 travailleurs (Èlebaeva, 2004), s'est toutefois brutalement tari à la suite des violences interethniques survenues en juin 2010 dont les principales victimes furent les populations ouzbèkes du Kirghizstan.

16 Pour un travail équivalent, le salaire des migrants d'Asie centrale correspond à 30 à $40 \%$ du salaire des Moscovites (Zajončkovskaâ, Mkrčân et Tûrûkanova, 2009).

17 Laruelle avance qu'entre 0,2 et 1 million de migrants ouzbékistanais travailleraient dans l'oblast' du Kazakhstan du Sud, entre 1 et 2 millions au Kazakhstan (Laruelle, 2010b), mais d'autres chercheurs estiment que le nombre de migrants présents au Kazakhstan oscillerait entre 0,7 et 1,2 million (Sadovskaâ, 2009).

18 La présence des migrants a suscité à plusieurs reprises des réactions d'hostilité dans les districts agricoles de la région de Djalal-Abad.

REMI 2010 (26) 3 pp. 31-57 
En Asie centrale, la circulation des personnes ne présentait aucune difficulté jusqu'à la décision des États de fonctionnaliser les nouvelles frontières internationales, à la fin des années 1990. Aujourd'hui des contraintes inédites affectent en revanche les mobilités transfrontalières : la frontière kazakhstano-ouzbékistanaise a plusieurs fois été partiellement fermée, de même que la frontière kirghizstano-ouzbékistanaise. Cependant, les autorités kazakhstanaises n'exigent pas des ressortissants ouzbékistanais la possession d'un visa bien que les migrants doivent se soumettre aux contrôles " pointilleux » des gardes-frontières, des douaniers et des policiers. Quoique des itinéraires clandestins existent dans l'oasis de Tachkent, la quasi-totalité des migrants entre donc légalement au Kazakhstan, mais nombre d'entre eux se voient contraints de verser un pot-de-vin (Alimbekova et Šabdenova, 2009). Au Kazakhstan, les démarches pour obtenir un permis de résidence ou une autorisation de travail de même que l'absence de document lors d'un contrôle de police nourrissent également une corruption à grande échelle (Trudovye..., 2005) ${ }^{19}$.

En raison de la proximité du Kazakhstan et du Kirghizstan, le transport routier est le principal moyen utilisé par les migrants. Toutefois, depuis la fermeture des frontières, aucune ligne régulière d'autocar n'assure plus de liaison transfrontalière. Pour s'affranchir de cette contrainte, une gare routière informelle a été implantée au nord du poste-frontière de Tchernaevka situé sur la route Tachkent-Chymkent. Reliée aux villes kazakhstanaises et russes, elle fait office pour Tachkent de gare routière internationale. Plus au sud, l'accès à la région du Jetisaï, appendice méridional du Kazakhstan, constitue un cas particulier, car la route Tachkent-Samarkand transitait par cette région cotonnière rattachée à la Steppe de la Faim. À l'automne, soir et matin, des centaines de ramasseurs de coton attendaient sur les bas-côtés de la route de monter dans une voiture, un minibus, un autocar ou un camion pour faire les quelques kilomètres séparant leur village des champs kazakhstanais. Pour limiter les mobilités transfrontalières, les autorités ouzbékistanaises ont décidé de fermer cette route en 2003 (Thorez, 2005). Mais certains taxis continuent de relier les bourgs ouzbékistanais aux villages kazakhstanais et s'improvisent intermédiaires entre les migrants et les employeurs, non sans prélever des sommes importantes aux migrants, avant de leur restituer leur passeport une fois la frontière franchie (Kamalova, 2005).

À l'échelle de l'Asie centrale, les migrations de travail connaissent une ampleur et une géographie inédites, malgré les entraves à la mobilité et la crise économique survenue en 2008. Alors que le Kazakhstan, premier pôle économique centre-asiatique, est devenu une destination majeure des migrants ouzbékistanais, ces flux de main-d'œuvre redéfinissent les relations entre l'Ouzbékistan et le Kazakhstan et remodèlent les équilibres géopolitiques régionaux.

\section{Tachkent, une capitale-relais ? Les migrations nationales}

À l'instar des migrations internationales, les migrations nationales sont devenues un phénomène social et économique majeur. Ces flux caractérisés par une surreprésentation des ruraux par rapport à leur poids dans la population et des femmes par rapport à leur

19 En 2005, un rapport estimait que les services de sécurité kazakhstanais avaient reçu plus de 15 millions de dollars des migrants, les sommes s'échelonnant en moyenne de 200 tenge $(1,5 \$)$ pour une absence de carte d'immigration à 1200 tenge $(9$ \$) pour l'obtention d'un enregistrement et à 32500 tenge (240\$) pour l'obtention d'un permis de résidence d'un an (Trudovye..., 2005). 
place dans les flux internationaux, sont polarisés par Tachkent (Maksakova, 2008) ${ }^{20}$. Si les villes de province, au premier rang desquelles Samarkand, reçoivent des migrants originaires des campagnes environnantes, Tachkent accueille les trois quarts des migrants intérieurs et recrute des travailleurs sur l'ensemble du territoire national (Maksakova, 2005 ; Abdullaev, 2008). Dans ce cadre, les régions méridionales (Surkhan-Daria, Kachka-Daria) et orientales (Ferghana) fournissent une part importante des migrants.

Les contrôles administratifs sur la mobilité résidentielle et professionnelle hérités de la période soviétique - les embauches sont toujours officiellement consignées dans le livret de travail des salariés - ne sont pas un obstacle à la circulation des travailleurs en Ouzbékistan $^{21}$. Pour leurs déplacements, ces derniers bénéficient d'une offre de transport diverse, malgré la contraction des réseaux aériens et ferroviaires domestiques depuis 1991 (Thorez, 2005) ${ }^{22}$. Alors que la compagnie ferroviaire O'zbekiston temir yo'llarl a mis en service des trains rapides sur l'axe Tachkent-Boukhara, le transport routier a vu son rôle croître dans la desserte du territoire national, notamment pour les liaisons interrégionales (Thorez, 2007b). Des autocars relient les régions occidentales et méridionales à Tachkent, mais les migrants empruntent également des taxis et des taxis collectifs interurbains, en particulier entre le Ferghana et Tachkent, car le franchissement du Tian-Chan est interdit aux autocars. Le soutien de l'État au secteur des transports, inspiré de la politique soviétique, permet en outre de pratiquer des tarifs contenus sur les trajets domestiques : en 2008, un billet de train entre Samarkand et Tachkent coûtait 9250 sum (cinq euros).

Parmi les travailleurs ruraux et provinciaux qui se rendent à Tachkent, certains connaissent préalablement leur destination professionnelle. C'est le cas de la plupart des hommes qui se déplacent à l'occasion d'un chantier de construction, au sein d'une équipe de travail dirigée par un brigadir. En général, ce dernier, qui est un membre de la famille, un ami ou une connaissance, se met d'accord en amont avec un intermédiaire ou un employeur. Dans ce cadre, migrent aussi bien des travailleurs inscrits dans la migration, qui sont souvent qualifiés, que des jeunes hommes désireux de gagner ponctuellement de l'argent en s'embauchant pour quelques semaines ${ }^{23}$. À Tachkent, cette mobilité entraîne une division géographique du travail sur les chantiers de construction : les brigades provinciales se chargent du gros œuvre, tandis que des équipes tachkentoises effectuent les finitions (Bazin, 2009). Toutefois, nombreux sont les migrants qui se déplacent à la recherche d'un travail non défini et qui rejoignent les rangs des mardikor, attendant dès l'aube, sur les bazars ou le long des routes, d'être embauchés pour la journée ou pour une durée un peu plus longue, comme soudeurs, bétonneurs, manœuvres, couvreurs ou agents d'entretien (Allahverdieva, 2007). Si des formes de travail journalier existaient

20 Parmi les déplacements saisonniers, la mobilité des travailleurs réquisitionnés pour la cueillette du coton apparaît rarement dans les travaux consacrés aux migrations de travail, en dépit de l'importance du phénomène.

21 Les migrations vers Tachkent sont également le fait d'étudiants attirés par la diversité de l'offre de formation proposée par les institutions d'enseignement supérieur.

22 Actuellement, un train par semaine relie Tachkent à Andijan, tandis que la liaison était assurée trois fois par jour à la veille de la partition de l'URSS.

23 En 2008, des jeunes étudiants venus du Khorezm pouvaient ainsi accumuler 150 à 200 dollars pendant l'été. 
durant la période soviétique, le mardikor est devenu au cours des dernières années une figure typique du monde du travail et un des visages des migrants. À Tachkent, des usines textiles emploient aussi des migrants d'origine rurale, moins payés et plus dociles que la main-d'œuvre locale, et organisent leur mobilité quotidienne (Bazin, 2009). Ces migrations pendulaires dessinent les contours d'un vaste bassin d'emploi autour de la capitale.

La place centrale occupée par Tachkent dans le système migratoire ouzbékistanais tient aussi à la fonction d'interface assurée par la ville entre les espaces migratoires nationaux et internationaux. Migrer vers Tachkent est souvent une première étape vers l'étranger. Les migrants y acquièrent des compétences professionnelles et linguistiques, à travers l'apprentissage du russe et nombreux sont ceux qui envisagent et organisent leur séjour dans la capitale comme un moment dans leur parcours migratoire individuel ou familial. Des familles ouzbékophones, reportant sur leurs enfants le projet de quitter l'Ouzbékistan les inscrivent ainsi dans des écoles où l'enseignement s'effectue en russe. Dans les grands quartiers résidentiels, tel Tchilanzar, des écoles ouzbèkes voient donc leurs effectifs diminuer tandis que la demande augmente fortement dans les écoles russes. Cela dit, la perspective de migrer oriente les choix scolaires partout en Ouzbékistan : dans toutes les régions, les effectifs des collèges préparant aux métiers du bâtiment (et de la restauration) croissent depuis plusieurs années (Abdullaev, 2008).

\section{Des qishloq à New York : un système migratoire hiérarchisé}

La mobilité des travailleurs ouzbékistanais prend des formes nationales ou internationales, mais les réseaux qui lient les lieux d'émigration et les lieux d'immigration structurent un système migratoire cohérent. Partir pour les États-Unis, la Corée du Sud, la Russie ou pour le Kazakhstan nécessite de mobiliser des ressources différentes que pour migrer vers Tachkent, mais les expériences migratoires présentent souvent des points communs. Les travailleurs migrants sont partout confrontés à des législations contraignantes qui reposent sur la délivrance de permis de résidence et de travail. De ce fait, la majorité des migrants habite et travaille illégalement à Tachkent tant il est difficile d'obtenir un enregistrement auprès des autorités compétentes. Dès lors de nombreux migrants, en particulier les mardikor, sont dépendants de la police, à l'instar de leurs homologues de Russie ou du Kazakhstan, et sont, selon Bazin, placés en situation « d'asservissement collectif » (2009). Pourtant, en Ouzbékistan, les relations avec la police ne semblent pas perçues comme un problème par les migrants (Abdullaev, 2008). Un autre parallèle peut être fait concernant les conditions d'hébergement tant la description des logements des travailleurs ouzbékistanais en Corée du Sud, en Russie ou au Kazakhstan fait écho à la vie quotidienne des migrants à Tachkent (Brusina, 2008 ; Massot, 2009). Une grande partie d'entre eux réside dans des appartements loués collectivement, au mépris des règles d'hygiène et des normes sociales, ou sur leur lieu de travail (Abdullaev, 2008) ${ }^{24}$. À Moscou, à Astana comme à Tachkent, les ouvriers du bâtiment, notamment ceux qui font le gros œuvre, habitent souvent sur les chantiers dans des baraquements et sont alors « captifs de leur lieu de travail » (Bazin, 2009).

24 De nombreux mardikor louent une place pour la nuit dans un appartement ou une maison qu'ils partagent avec d'autres migrants, souvent hommes et femmes mélangés. En 2007, ils dépensaient en moyenne 500 sum par jour pour se loger (Allahverdieva, 2007). 
Tableau 2 : La hiérarchie des migrations de travail ouzbékistanaises

\begin{tabular}{|c|c|c|c|}
\hline Hiérarchie & \multicolumn{2}{|c|}{ Nature des migrations } & Destinations \\
\hline \multirow{7}{*}{-} & \multirow{2}{*}{\multicolumn{2}{|c|}{ Nationales }} & Villes de province \\
\hline & & & Tachkent \\
\hline & \multirow{7}{*}{ Internationales } & \multirow[b]{2}{*}{ Régionales } & Kazakhstan du Sud \\
\hline & & & $\begin{array}{l}\text { Kazakhstan : Almaty, Astana, régions } \\
\text { caspiennes }\end{array}$ \\
\hline & & Postcoloniales & Russie \\
\hline & & \multirow{4}{*}{ Globales } & Émirats arabes unis \\
\hline & & & Corée du Sud \\
\hline \multirow{2}{*}{+} & & & Europe \\
\hline & & & Amérique du Nord \\
\hline
\end{tabular}

Les trajectoires migratoires s'inscrivent dans une hiérarchie des destinations qui dépend du niveau d'accessibilité, des opportunités économiques et du prestige des lieux d'immigration (Tableau 2). Les itinéraires des migrants témoignent de la fluidité qui existe entre les sous-ensembles de l'espace migratoire, en particulier au sein de l'espace postsoviétique, et qui donne sa cohérence au champ migratoire. Si la majorité des migrants privilégie des stratégies fondées sur la stabilité de leur implantation à l'étranger, la perspective d'améliorer la réussite économique et sociale de la migration conduit certains d'entre eux à se diriger vers de nouvelles destinations plus profitables bien que moins accessibles. Inversement, après avoir travaillé plusieurs saisons en Russie, des migrants peuvent choisir de se rendre au Kazakhstan, car sa proximité géographique permet de revenir plus fréquemment au qishloq, par exemple après une naissance ou durant une période de deuil. À cet égard, il convient d'appréhender avec souplesse la hiérarchie des destinations d'autant que les choix migratoires s'inscrivent dans des contextes locaux qui participent à l'orientation géographique de la mobilité des travailleurs. Dans les villes comme dans les villages, les réseaux de sociabilité mobilisés au cours de la migration contribuent à déterminer la destination des migrants : les habitants d'un quartier, d'un village voire d'un district tendent à se diriger vers les mêmes lieux (Reeves, 2010).

\section{LES MIGRATIONS DE TRAVAIL, UNE GÉOPOLITIQUE « PAR LE BAS »}

\section{Ambiguïtés ouzbékistanaises : entraver ou accompagner les migrations?}

Malgré l'ampleur des flux, les autorités refusent de faire de la question migratoire un élément important de leurs politiques étrangère et économique, à la différence du Tadjikistan et du Kirghizstan. Alors que l'Ouzbékistan n'a pas signé les conventions 
internationales élaborées sous l'égide de l'ONU sur les migrations de travail, l'État semble apporter un soutien limité à ses ressortissants émigrés. Ainsi, il n'existe pas d'accord bilatéral entre l'Ouzbékistan et le Kazakhstan sur le statut des migrants de travail (Laruelle, 2010b). En Russie, aucun réseau associatif comparable à ceux des communautés tadjikistanaises ou kirghizstanaises n'assure l'interface entre les migrants et les autorités ouzbékistanaises et russes. Cette attitude renvoie au refus de l'État de reconnaître combien l'externalisation de la main-d'œuvre modèle la société et l'économie (Laruelle, 2010a). À bien des égards, elle traduit également la volonté du pouvoir de conduire une politique de limitation des migrations, conçue au nom d'enjeux sécuritaires comme un outil de contrôle de la population et du territoire.

Depuis l'essor des migrations de travail, la législation a ainsi été amendée à plusieurs reprises au détriment de la liberté de circuler et de travailler à l'étranger (Ilkhamov, 2006). En 2003, l'État a décidé de soumettre à l'autorisation d'une commission interministérielle le droit de travailler à l'étranger, avant d'introduire de nouveaux dispositifs contraignants en 2005 et en 2007. Outre le contrôle de la mobilité, ces mesures ont permis à l'administration de s'impliquer davantage dans la régulation d'un secteur très lucratif, la complexité des démarches ouvrant un vaste espace de négociation informel entre migrants et représentants des services de l'État. Plus largement, l’État semble tenter de contenir un processus qui questionne sa souveraineté et sa légitimité (Massot, 2009).

La politique migratoire ne se limite cependant pas à cet aspect. Les autorités disposent d'une part d'une connaissance fine du phénomène migratoire, grâce aux informations recueillies à l'échelle des mahalla et au travail d'analyse effectué par les administrations centrales. D'autre part, malgré la discrétion des représentants des ministères et des administrations, de nombreux documents officiels, émanant par exemple du Praesidium du Cabinet des ministres, abordent clairement la question des migrations de travail et de leurs conséquences sur la situation économique et sociale. Enfin, la prudence affichée par l'État vis-à-vis des migrations n'a pas empêché le pays de signer plusieurs accords bilatéraux. En 1995, un accord a été conclu avec la Corée du Sud, impliquant notamment la Fédération coréenne des petites et moyennes entreprises. En 2007, 1'Ouzbékistan et la Russie ont signé plusieurs accords, ratifiés en 2009, qui portent sur la défense des droits des travailleurs-migrants, sur la lutte contre les migrations clandestines et sur le rapatriement des migrants illégaux expulsés. Ces accords sont censés déboucher sur un progrès significatif du statut des travailleurs ouzbékistanais en Russie. La participation de l'Ouzbékistan à la structure interétatique qui regroupe depuis 2008 les administrations des migrations des pays de la CEI doit également permettre d'améliorer les conditions de circulation, de résidence et de travail des migrants (Maksakova, 2009).

L'implication des autorités ouzbékistanaises dans la régulation des flux migratoires repose aussi sur le rôle joué par les entreprises de transport détenues par l'État dans la circulation internationale des migrants. Or, depuis le début des années 2000, Uzbekistan Airways et Uzbekistan Railways ont orienté leur développement sur les liaisons entre l'Ouzbékistan et la Russie (Figures 2 et 3). En 2000, Uzbekistan Airways assurait vingtsept vols hebdomadaires à destination de treize villes russes ; en 2008, elle opérait soixantetrois vols hebdomadaires en direction de seize aéroports de Russie. Après avoir été un outil d'affirmation de la souveraineté nationale dans les années 1990, Uzbekistan Airways 
contribue aujourd'hui à l'intensification des circulations migratoires. Ce repositionnement stratégique possède une dimension économique évidente en répondant à l'augmentation de la demande de transport à destination de la Russie. Mais il participe aussi d'une volonté politique qui vise non seulement à tolérer la mobilité des migrants, mais également à accompagner voire à encourager le développement des migrations de main-d'œuvre.

\section{Les dépendances postcoloniales d'un pays du Sud ?}

La nouvelle territorialité des populations centre-asiatiques est un des vecteurs d'intégration de l'Ouzbékistan dans l'espace mondialisé. Les flux migratoires contribuent à l'ouverture internationale de la société et de l'économie de même qu'à la réévaluation du positionnement géopolitique et géoéconomique du pays et notamment à la reformulation des relations entretenues avec la Russie. Le rapprochement diplomatique entre l'Ouzbékistan et la Russie opéré en 2005 a souvent été relié à la condamnation occidentale de la répression de l'insurrection d'Andijan. Mais ce virage politique renvoie également à la réactivation des dynamiques d'intégration régionale suscitée par les migrations de maind'œuvre.

Cette « géopolitique par le bas » repose sur l'intensification des migrations de travail et sur le développement des réseaux de transports. Inscrite dans un schéma postcolonial classique, elle marque une évolution profonde des relations entre l'Ouzbékistan et la Russie, en raison de la dépendance économique et sociale qui naît des flux migratoires. La mobilité des travailleurs s'accompagne en effet d'importants transferts de capitaux qui irriguent l'économie domestique. Chaque année, plusieurs milliards de dollars (officiellement, 3 milliards de dollars en 2008) pénètrent sur le territoire ouzbékistanais par l'entremise des migrants (Maksakova, 2009). Cette opération est assurée par des entreprises de transfert d'argent, mais les experts estiment qu'une somme au minimum équivalente au capital officiellement transféré est illégalement transmise par les migrants (Rynok..., 2007). Différents réseaux informels, notamment commerçants, permettent d'expédier de l'argent ; des biens (hifi, électroménager, prêt-à-porter) peuvent aussi être envoyés, en particulier depuis la Corée du Sud, pour être revendus en Ouzbékistan par la famille. Ces flux de capitaux, qui représentent selon les estimations entre $10 \%$ et $25 \%$ du PIB, instituent à l'échelle des familles une dépendance à l'endroit de la Russie (et du Kazakhstan), laquelle se substitue aux formes d'interdépendance construites entre les différentes régions soviétiques à l'échelle des administrations et des entreprises.

L'évolution de la géographie des liaisons aériennes entre l'Ouzbékistan et la Russie depuis la disparition de l'URSS rend également compte du changement de statut de l'Ouzbékistan. Malgré une croissance régulière durant les années 2000, l'offre de transport apparaît moins développée aujourd'hui qu'à la fin de la période soviétique, quand Tachkent était le second aéroport d'URSS pour les liaisons moyen et long-courrier (Figures 2, 3 et 4). Mais davantage de villes d'Ouzbékistan sont aujourd'hui reliées à la Russie qu'en 1985, quand les liaisons directes étaient pourtant privilégiées au détriment des hubs dans le système de transport soviétique (Sagers et Maraffa, 1990 ; 1991). Cette transformation du réseau, qui correspond à l'établissement de lignes régulières entre tous les centres régionaux et Moscou, est parallèle à la rétraction de l'espace desservi en Russie depuis l'Ouzbékistan (Figures 2, 3 et 4). Cette dynamique affecte la desserte des 


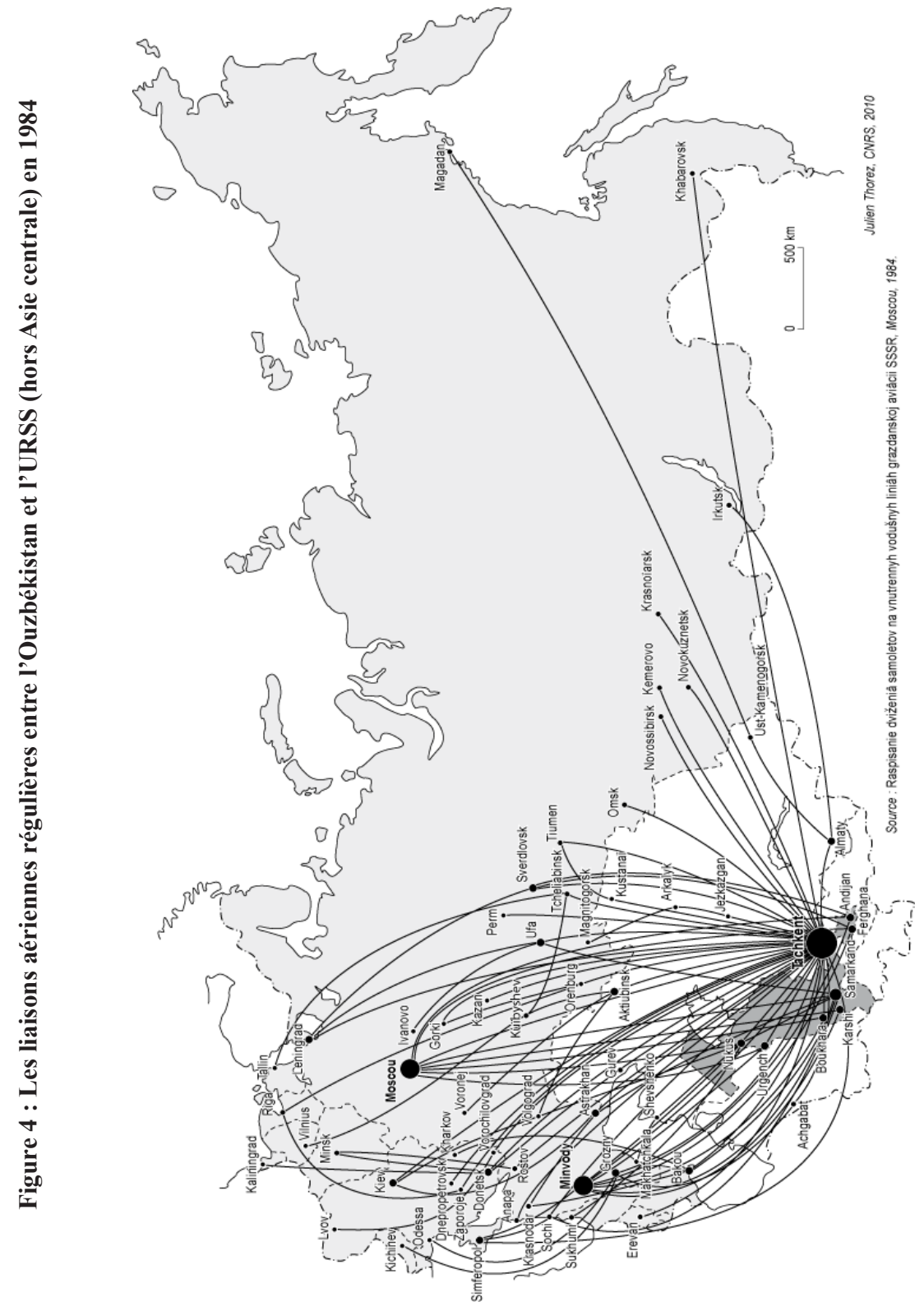


villes moyennes, mais surtout celle des régions touristiques. Au milieu des années 1980, Minvody, située sur le piémont septentrional du Caucase, au cœur d'une grande région thermale, était reliée à huit aéroports ouzbékistanais et était ainsi après Moscou l'aéroport le mieux connecté à l'Ouzbékistan ; aujourd'hui, un vol hebdomadaire est opéré depuis Tachkent par Uzbekistan Airways (Figures 2 et 4). Cette mutation du réseau aérien répond à la reformulation de la demande de transport qui conduit les mobilités laborieuses à se substituer aux mobilités touristiques. Cette évolution structurelle révèle la transformation profonde de l'architecture géoéconomique et géopolitique du monde post-soviétique et traduit le glissement de l'Ouzbékistan vers le Sud.

\section{CONCLUSION}

Les migrations de travail saisonnières et temporaires, qui furent souvent interprétées comme une réponse à la crise post-soviétique, sont désormais ancrées durablement dans les pratiques individuelles et collectives ${ }^{25}$. La société ouzbékistanaise, profondément transformée, s'inscrit à moyen et long terme dans cette forme de mobilité inédite : une enquête réalisée pour l'administration a montré que $85 \%$ des familles interrogées ne concevaient pas leur avenir sans la migration de l'un de ses membres (Rynok..., 2007) ; une première génération de migrants transmet son expérience de l'étranger et ses réseaux professionnels à des successeurs dont la formation en Ouzbékistan est de plus en plus déterminée par la demande extérieure. Si la crise économique survenue en 2008-2009 a réduit l'activité en Russie et au Kazakhstan, de sorte que des migrants ont annulé ou anticipé leur retour au pays ou bien retardé leur départ, les flux migratoires s'intensifient de nouveau, de même qu'au Tadjikistan ou au Kirghizstan. En une décennie, l'Ouzbékistan est devenu un pays de migrants et de familles de migrants, dont le développement repose de plus en plus sur l'externalisation de la main-d'œuvre, en contradiction avec la politique économique protectionniste suivie depuis l'indépendance.

Les migrations de main-d'œuvre contribuent, parallèlement à l'action diplomatique conduite par les autorités, à redéfinir le positionnement international de l'Ouzbékistan, en interrogeant son modèle de développement et sa place dans le monde contemporain. Polarisées par la Russie, elles tendent à ancrer l'Ouzbékistan dans une situation post-coloniale classique. Mais leur apparition puis leur généralisation illustrent aussi la spécificité de la trajectoire suivie depuis l'indépendance par l'Ouzbékistan (et par les pays du Caucase et de l'Asie centrale); son itinéraire post-soviétique s'apparentant à une forme de décrochage du Nord vers le Sud.

25 Des migrants « centre-asiatiques » d'Ouzbékistan envisagent une installation définitive à l'étranger, mais ils restent minoritaires. 


\section{Références bibliographiques}

ABDULLAEV E.V. (Éd.) (2008) Trudovaâ migraciâ v respublike Uzbekistan [Les migrations de travail en Ouzbékistan], Tashkent, UNDP, 204 p.

ALIAKBEROVA N.M., GOL'DFARB B.Â. et ÈRGAŠEV A. (1989) Razmeŝenie naseleniâ ferganskoj doliny (demografičeskij aspekt) [La répartition de la population de la vallée du Ferghana (aspects démographiques)], Tachkent, Fan, 98 p.

ALIMBEKOVA G.T. et ŠABDENOVA A.A. (2009) Zanâtost' trudâsihihsâ-migrantov v neformal'noj èkonomike Kazahstana [L'emploi des migrants de travail dans l'économie informelle au Kazakhstan], Almaty, IOM, 40 p.

ALLAHVERDIEVA L.M.(2007) Neformal'nye rynki truda i problemy vnutrennoj migracii naseleniâ Uzbekistana [Les marchés informels du travail et les migrations intérieures en Ouzbékistan], in Naselenie Uzbekistana: 15 let nezavisimogo razvitiâ, Tachkent, Centr social'no-èkonomičeskih issledovanij, UNFPA, pp. 71-76.

ARTYKOVA D.A. (2007) Feminizaciâ trudovoj migracii [La féminisation des migrations de travail], in Naselenie Uzbekistana: 15 let nezavisimogo razvitiâ, Tashkent, Centr social'noèkonomičeskih issledovanij, UNFPA, pp. 142-144.

BATER James H. (1989) The Soviet Scene. A Geographical Perspective, London, Edward Arnold, $304 \mathrm{p}$.

BAZIN Laurent (2009) Paysans ouvriers, in Laurent Bazin, Monique Selim et Bernard Hours Éds, L'Ouzbékistan à l'ère de l'identité nationale, Paris, L'Harmattan, 368 p.

BELOUIN Stéphanie (2010) Projets et stratégies migratoires de femmes qualifiées à Tachkent, in Marlène Laruelle Éd., Dynamiques migratoires et changements sociétaux en Asie centrale, Paris, Pétra, pp. 297-318.

BRAUX Adeline (2009) Politique migratoire et gestion de la diversité culturelle en Russie : l'exemple de Moscou, Paris, Les études du CERI, 158, 37 p.

BRUSINA O. (2008) Migranty iz Srednej Azii v Rossii: ètapy i pričiny priezda, social'nye tipy, organizacii diaspor [Les migrants d'Asie moyenne en Russie : étapes et causes migratoires, profil social et organisation des diasporas], Vestnik Evrazii, 2 (40), pp. 66-95.

ÈLEBAEVA A.B. (2004) Trudovye migracii v Kyrgyzstane [Les migrations de travail au Kirghizstan], Central'naâ Aziâ i Kavkaz, 27, pp. 78-86.

FERRANDO Olivier (2010) Politiques diasporiques et flux migratoires : les États-nations et leurs minorités co-ethniques de l'étranger, in Marlène Laruelle Éd., Dynamiques migratoires et changements sociétaux en Asie centrale, Paris, Pétra, pp. 49-76.

KAMALOVA N. (2005) Voprosy migracii v Džizakskom viloâte [La question migratoire dans la région de Djizakh], in D.S. Azimova et E.V. Abdullaev Éds., Trudovaâ migraciâ: social'nye $i$ pravovye aspekty [Les migrations de travail : aspects sociaux et juridiques], Tachkent, pp. 29-34.

KOROBKOV A.V. and ZAIONCHKOVSKAIA Ž.A. (2004) The Changes in the Migration Patterns in the Post-Soviet States: The First Decade, Communist and Post-Communist Studies, 37, pp. 481-508.

ILKHAMOV A. (2006) Geographic Mobility of Uzbeks: The Emergence of Cross-national Communities vs. Nation-state Control, Paper for NBR Conference, Seattle, 23 p., http://www. nbr.org/Downloads/pdfs/PSA/Uzk_Conf06_Ilkhamov.pdf.

IVATOV I. (1994) Razvitie dehkanskogo rynka v Uzbekistane [Le développement des marchés paysans en Ouzbékistan], Tachkent, Fan, 109 p.

Labour Migrants from Uzbekistan in the Southern Regions of Kazakhstan (2005) Almaty, IOM, $72 \mathrm{p}$.

LAITIN David (1998) Identity in Formation, the Russian-speaking Population in the Near Abroad, Ithaca-London, Cornell University Press, $417 \mathrm{p}$ 
LARUELLE Marlène (2007) Central Asian Labour Migrants in Russia: The "Diasporization" of the Central Asian States?, China and Eurasia Forum Quarterly, 5 (3), pp. 101-119.

LARUELLE Marlène (Éd.) (2010a) Dynamiques migratoires et changements sociétaux en Asie centrale, Paris, Pétra, $320 \mathrm{p}$.

LARUELLE Marlène (2010b) Le Kazakhstan, nouveau carrefour migratoire d'Asie centrale, in Marlène Laruelle Éd., Dynamiques migratoires et changements sociétaux en Asie centrale, Paris, Pétra, pp. 77-98.

MAKSAKOVA L.P. (1986) Migraciâ naseleniâ v Uzbekistane [Les migrations de population en Ouzbékistan], Tachkent, Uzbekistan, 208 p.

MAKSAKOVA L.P. (2001) Migraciâ naseleniâ: problemy regulirovaniâ [La régulation des migrations de population], Tachkent, Eldinur, $185 \mathrm{p}$.

MAKSAKOVA L.P. (2005) Trudovaâ migraciâ naseleniâ Uzbekistana: tendencii i regional'nye osobennosti [Les migrations de travail en Ouzbékistan : dynamiques et spécificités régionales], in D.S. Azimova et E.V. Abdullaev Éds., Trudovaâ migraciâ. social'nye i pravovye aspekty, Tachkent, pp. 6-19.

MAKSAKOVA L.P. (2008) Vnutrennaâ migraciâ naseleniâ Uzbekistana: sociologičeskie aspekty [Les migrations intérieures en Ouzbékistan : une approche sociologique], in E.V. Abdullaev Éd., Trudovaâ migraciâ v respublike Uzbekistan, Tachkent, UNDP, pp. 44-56.

MAKSAKOVA L.P. (2009) Uzbekistan v sisteme meždunarodnyh migracii [L'Ouzbékistan dans le système migratoire international], in Ž.A Zajonckovkaâ et G. S. Vitkovskaâ Éds., Postsovetskie transformacii: otraženie v migraciâh, Moscou, Adamant, pp. 323-349.

MASSOT Sophie (2009) Enjeux politiques et identitaires de la migration internationale des Ouzbeks vers trois pôles urbains (Moscou, Séoul, New York), Thèse de doctorat, Sciences Po Paris, 484 p.

Migration in Central Asia: Challenges and Prospects (2005), Almaty, Unesco, 202 p.

MULLÂDŽANOV I.R. et UBAJDULLAEVA R.A. (Éds.) (1988) Razvitie narodonaseleniâ $i$ problemy trudovyh resursov republik Srednej Azii [Croissance démographique et ressources laborieuses en Asie moyenne], Tachkent, Fan, 164 p.

Naselenie Uzbekistana: 15 let nezavisimogo razvitiâ [La population de l'Ouzbékistan : quinze ans de développement indépendant] (2007), Tachkent, Centr social'no-èkonomičeskih issledovanij, UNFPA, $271 \mathrm{p}$.

OLIMOVA S.K. et SADOVSKAÂ E.Û. (2005) Trudovaâ migraciâ v stranah Central'noj Azii, Rossijskoj Federacii, Afganistane i Pakistane [Les migrations de travail en Asie centrale, en Russie, en Afghanistan et au Pakistan], Almaty, EC, IOM, 146 p.

PATNAIK Ajay (1995) Agriculture and Rural Out-migration in Central Asia (1960-1991), EuropeAsia Studies, 47 (1), pp. 147-169.

RABALLAND Gaël (2005) L'Asie centrale ou la fatalité de l'enclavement, Paris, L'Harmattan, IFEAC, $355 \mathrm{p}$.

REEVES Madeleine (2010) Migrations, masculinité et transformation de l'espace social dans la vallée de Sokh, in Marlène Laruelle Éd., Dynamiques migratoires et changements sociétaux en Asie centrale, Paris, Pétra, pp. 217-245.

Rynok meždunarodnyh denežnyh perevodov $v$ Uzbekistane [Le marché des transferts d'argent internationaux en Ouzbékistan] (2007), Tachkent, Centr po soveršenstvovaniû antimonopol'noj politiki, $83 \mathrm{p}$.

SADOVSKAÂ E.Û. (2009) Kazahstan v central'noaziatskoj migracionnoj subsisteme [Le Kazakhstan dans le sous-système migratoire centre-asiatique], in Ž.A Zajonckovkaâ et G.S. Vitkovskaâ Éds., Postsovetskie transformacii: otraženie v migraciâh, Moscou, Adamant, pp. 279-322.

SAGERS Matthew J. and MARAFFA Thomas (1990) Soviet Air Passenger Transportation Network, The Geographical Review, 80 (3), pp. 266-278.

SAGERS Matthew J. and MARAFFA Thomas (1991) The Spatial Structure of Air Passenger Services for Kiev and Tashkent, Soviet Geography, 32 (5), pp. 314-326. 
TARASOVA N.V. (1985) Problema povyšeniâ migracionnoj aktivnosti naseleniâ [L'accroissement de l'activité migratoire], in Sovremennye problemy migracii [Les problèmes contemporains des migrations], Moscou, Mysl', 1985, pp. 47-63.

TAŠBAEVA T.H. et SAVUROV M.D. (1989) Novoe i tradicionnoe v bytu sels'koj sem'i Uzbekov [Nouveauté et tradition dans le mode de vie des familles rurales ouzbèkes], Tachkent, Fan, $162 \mathrm{p}$.

THOREZ Julien (2005) Flux et dynamiques spatiales en Asie centrale - Géographie de la transformation post-soviétique, Thèse de doctorat, Université Paris X-Nanterre, $570 \mathrm{p}$.

THOREZ Julien (2007a) Itinéraires du déracinement - L'essor des migrations de travail entre l'Asie centrale et la Russie, Espaces, populations et sociétés, 1, pp. 59-71.

THOREZ Julien (2007b) La construction territoriale de l'indépendance : réseaux et souveraineté en Asie centrale post-soviétique, Flux, 70, pp. 33-48.

THOREZ Julien (2008) Bazars et routes commerciales en Asie centrale. Transformation post-soviétique et «mondialisation par le bas », Revue Européenne des Migrations Internationales, 24 (3), pp. 167-189.

THOREZ Julien (2009) « Хорошо где нас нет »! L'émigration des « Russophones » d'Asie centrale, EchoGéo, 20 p., http://echogeo.revues.org/index10890.html.

TINGUY Anne de (2004) La grande migration. La Russie et les Russes depuis l'ouverture du rideau de fer, Paris, Plon, $662 \mathrm{p}$.

TOPILIN A.V. (1975) Territorial'noe pereraspredelenie trudovyh resursov SSSR [La redistribution territoriale de la main-d'œuvre en URSS], Moscou, Ekonomika, 1975, 159 p.

Trudovye migranty iz Uzbekistana v iužnyh regionah Respubliki Kazahstan [Les migrants de travail d'Ouzbékistan dans les régions méridionales du Kazakhstan] (2005), Almaty, MOM, 37 p.

ZAJONČKOVSKAÂ Ž.A. (1996) Istoričeskie korni migracionnoj situacii v Srednej Azii [Les racines historiques de la situation migratoire en Asie moyenne], in G.S. Vitkovskaâ Éd., Migraciâ russkoâzyčnogo naseleniâ iz Central'noj Azii: pričiny, posledsvtiâ, perspektivy [L'émigration des populations russophones d'Asie centrale : causes, conséquences, perspectives], Moscou, Carnegie, pp. 41-64.

ZAJONČKOVSKAÂ Ž.A (2008) Migracia naseleniâ iz stran Central'noj Azii [Les migrations de population depuis les pays d'Asie centrale], in E.V. Abdullaev Éd., Trudovaâ migraciâ v respublike Uzbekistan, Tashkent, UNDP, pp. 24-34.

ZAJONČKOVSKAÂ Ž.A., MKRČÂN N. et TÛRÛKANOVA E. (2009) Rossiâ pered vyzovami immigracii [La Russie face à l'appel de l'immigration] in Ž.A Zajonckovkaâ et G.S. Vitkovskaâ Éds., Postsovetskie transformacii: otraženie v migraciâh, Moscou, Adamant, pp. 9-62.

ZAJONČKOVSKAÂ Ž.A. et VITKOVSKAÂ G.S. (Éds.) (2009) Postsovetskie transformacii: otraženie v migraciâh [Les transformations post-soviétiques au miroir des migrations], Moscou, Adamant, $412 \mathrm{p}$.

ZÛZIN D.I (1983) Pričini nizkoj mobil'nosti korennogo naseleniâ respublik Srednej Azii [Les causes de la faible mobilité des populations autochtones d'Asie moyenne], Sociologičeskie issledovaniâ, 1, pp. 109-118. 


\title{
La mobilité des migrants d'Ouzbékistan : transports, frontières et circulation migratoire
}

\author{
Julien THOREZ
}

\begin{abstract}
Depuis la fin des années 1990, la territorialité des populations centre-asiatiques d'Ouzbékistan se transforme rapidement, suite à l'émergence de migrations de travail, saisonnières et temporaires, qui questionnent le modèle de développement suivi depuis l'indépendance. Cette mobilité inédite, devant laquelle les autorités ont adopté une attitude ambiguë, s'inscrit dans des dynamiques globales et dans des dynamiques post-coloniales : structuré par des liaisons aériennes, ferroviaires et routières, l'espace de circulation des migrants s'étend de l'Asie orientale à l'Amérique du Nord, mais les principales destinations des travailleurs ouzbékistanais, qui sont majoritairement des hommes, sont la Russie et, secondairement, le Kazakhstan. Instaurant de nouvelles formes de dépendances, ces flux massifs renvoient à la trajectoire originale, du Nord vers le Sud, poursuivie par l'Ouzbékistan.
\end{abstract}

\section{Uzbekistan Migrants Movement: Transportation, Borders and Migratory Circulation}

\author{
Julien THOREZ
}

Since the late 1990s, the territoriality of Central Asian populations of Uzbekistan is changing, due to the emergence of labour migrations. Facing this seasonal and temporary mobility, Uzbekistanis authorities adopted an ambiguous attitude, since the new territorial and social practices raise questions about the development model followed after independence. This unprecedented mobility is a part of global and post-colonial dynamics: the migratory space, which is structured by airline, train and bus networks, is stretching from East Asia to North America. Nevertheless, the main destinations of Uzbekistanis workers, who are mostly men, are Russia and, secondarily, Kazakhstan. Introducing new forms of dependency, these massive flows refer to the original trajectory pursued by Uzbekistan, from the "North" to the "South".

\section{La movilidad de los emigrantes de Uzbekistán: transportes, fronteras y circulación migratoria Julien THOREZ}

Desde el final de los años 1990, la territorialidad de las poblaciones centroasiáticas de Uzbekistán se transforma rápidamente dado a la emergencia de migraciones de trabajo, estacionales y temporales, que ponen en tela de juicio el modelo de desarrollo seguido desde le independencia. Esta movilidad inédita, ante la cual las autoridades han adoptado una actitud ambigua, entra en la marco de dinámicas globales y postcoloniales: estructurado por conexiones aéreas, ferroviarias y de carreteras, el espacio de circulación de los migrantes se extiende desde Asia oriental hasta América del norte, pero las principales destinaciones de los trabajadores uzbecos, que son hombres en su mayoría son, Rusia sobre todo y luego Kazajstán. Al instaurar nuevas formas de dependencias, esos flujos masivos remiten a la trayectoria original, del Norte hacia el Sur, seguida por Uzbekistán. 


\title{
Мобильность мигрантов из Узбекистана: транспорт, границы и миграционные потоки

\author{
Julien THOREZ
}

\begin{abstract}
География населения Узбекистана центрально-азиатского происхождения изменилась с конца 1990-х годов, после появления и роста трудовых миграций, в том числе сезонных и временных. Новые социальные и территориальные практики и явления вызывают вопрос о пост-советской модели развития. Эти новые потоки, отношение к которым у властей двусмысленное, вписываются в картину глобальных и пост-колониальных процессов. Пространство миграций жителей Узбекистана, в основном мужчин, простирается от Восточной Азии до Северной Америки, но большинство из них работает в России, а также в Казахстане, куда они ездят самолётом, поездом или автобусом. Трудовые миграции порождают новые виды зависимости и меняют положение Узбекистана в глобальном мире: с его прежней «северной» принадлежности на «южную».
\end{abstract}


vol. $26-\mathrm{n}^{\circ} 3$ (2010)

Migrations en Asie centrale et au Caucase

Sophie Massot

\title{
Le retour des migrants ou l'émergence des « nouveaux Ouzbeks »: les effets d'un rite de transition
}

\author{
Avertissement \\ Le contenu de ce site relève de la législation française sur la propriété intellectuelle et est la propriété exclusive de \\ l'éditeur. \\ Les œuvres figurant sur ce site peuvent être consultées et reproduites sur un support papier ou numérique sous \\ réserve qu'elles soient strictement réservées à un usage soit personnel, soit scientifique ou pédagogique excluant \\ toute exploitation commerciale. La reproduction devra obligatoirement mentionner l'éditeur, le nom de la revue, \\ l'auteur et la référence du document. \\ Toute autre reproduction est interdite sauf accord préalable de l'éditeur, en dehors des cas prévus par la législation \\ en vigueur en France.
}

\section{revues.org}

Revues.org est un portail de revues en sciences humaines et sociales développé par le Cléo, Centre pour l'édition électronique ouverte (CNRS, EHESS, UP, UAPV).

\section{Référence électronique}

Sophie Massot, "Le retour des migrants ou l'émergence des " nouveaux Ouzbeks » : les effets d'un rite de transition », Revue européenne des migrations internationales [En ligne], vol. 26 - n³ | 2010, mis en ligne le 01 décembre 2013, consulté le 03 janvier 2014. URL : http://remi.revues.org/5208 ; DOI : 10.4000/remi.5208

Éditeur : Université de Poitiers

http://remi.revues.org

http://www.revues.org

Document accessible en ligne sur : http://remi.revues.org/5208

Ce document est le fac-similé de l'édition papier.

(c) Université de Poitiers 


\title{
Le retour des migrants ou l'émergence des «nouveaux Ouzbeks »: les effets d'un rite de transition
}

\author{
Sophie MASSOT ${ }^{1}$
}

\section{INTRODUCTION}

T es migrations de travail des Ouzbeks ${ }^{2}$ vers Séoul, Moscou et New York ont _fait l'objet de recherches ${ }^{3}$ menées de 2007 à 2010 (Massot, 2009). Nous avons montré que les migrants aux différentes étapes du parcours migratoire se trouvent au centre d'une nébuleuse socio-familiale dont les intérêts économiques et les enjeux de pouvoir sont complexes et n'agissent pas toujours avec la même prégnance. Il convient

1 Docteur en Science Politique, ATER de Science Politique à l'Université de la Réunion, 11 bis rue Guy Saint Lambert, 97411 Bois de Nèfles-Saint Paul ; sophiealdric@yahoo.fr

2 Il convient ici de distinguer les termes « ouzbek » (qui renvoie à la nationalité) et « ouzbékistanais » (qui renvoie à la citoyenneté). J'ai choisi de me concentrer sur l'émigration des Ouzbeks d'Ouzbékistan, et non sur l'ensemble des Ouzbékistanais. Chaque nationalité a sa spécificité, ce qui induit des processus migratoires difficilement comparables. Chacun a un lien particulier avec l'Ouzbékistan, qui peut représenter un pays d'origine, d'accueil, d'exil, de destination volontaire ou forcée. Il est ardu de mêler dans une même analyse la mobilité des Ouzbékistanais de nationalité allemande, ouzbèke ou coréenne. Par ailleurs, les Ouzbékistanais ne sont pas tous perçus de la même façon à l'étranger. Ainsi, un Ouzbékistanais russe et un Ouzbékistanais ouzbek ne rencontreront pas les mêmes difficultés à Moscou. Enfin, bien que cela soit sujet à débat, il semble que chaque groupe ethnique cultive sa propre culture, qui peut avoir une influence considérable sur la manière d'envisager la migration, puis sur la période migratoire. Une autre raison à ce choix de m'intéresser à la nationalité ouzbèke est qu'elle est majoritaire en Ouzbékistan, bien que les chiffres officiels soient à manier avec précaution. L'impact des migrations des Ouzbeks sur leur pays d'origine est peut-être plus sensible, surtout dans un contexte où une politique d'ouzbékisation a été menée.

3 Dans le cadre du doctorat à l'IEP de Paris, plusieurs terrains ont été abordés : recherche préparatoire en Ouzbékistan (juillet-août 2005) qui a permis de mesurer les enjeux des migrations internationales, puis Moscou (avril-mai 2006), Séoul (juillet-août 2006), New York (février-mars 2007) et enfin Ouzbékistan (avril-mai 2007 et janvier-février 2009). Retour en Ouzbékistan en janvier-février 2009 afin de mesurer l'impact des retours des migrants. 
alors de considérer ces diverses relations nouées par les migrants selon un cadre spatiotemporel qui permet d'en mesurer l'évolution : il faut distinguer la période liminaire en Ouzbékistan, qui précède le départ, de la période où les migrants se trouvent dans le pays d'accueil, et enfin lorsqu'ils reviennent à leur point de départ; la plupart des migrations étant temporaires. De même, il faut distinguer les liens de nature familiale et ceux de nature socio-économique.

Il est possible d'analyser ce phénomène à l'aune de ce que j'ai appelé un « rite de transition », notion forgée à partir des travaux de Van Gennep (1981) sur les rites de passages et de Bourdieu (1982) sur les rites d'institution. En effet, la migration effective représente un rite qui doit s'accomplir seul après la mise en place par le réseau familial des conditions de sa réalisation. Dès lors, lorsque les migrants séjournent dans le pays d'accueil, ils doivent se prouver à eux-mêmes et à leur famille qu'ils appartiennent bien à leur communauté afin de refonder leur identité ouzbèke - leur ouzbékité/o'zbekchilik (Massot, 2009). La rupture avec les réseaux d'origine serait donc une condition nécessaire à la validation de la migration par le groupe d'origine, et à la réintégration du migrant dans ce réseau, qui implique une prise de pouvoir socio-économique.

Nous nous intéresserons ici au retour des migrants, en nous demandant quels sont les effets de ce rite de transition lorsque la boucle migratoire a été réalisée : les migrants changent-ils de statut social en fonction de leur nouveau pouvoir économique ? Y a-t-il création de nouveaux réseaux de solidarité dont ils seraient le pivot? Leur propre perception de l'ouzbékité est-elle transformée par le parcours migratoire ? S'inscrivent-ils dans de nouvelles logiques migratoires?

Nous montrerons tout d'abord que les effets sociaux du rite de transition migratoire induisent un bouleversement des statuts au sein de la parenté, en nous demandant s'il y a importation de modèles culturels et familiaux occidentaux, puis nous analyserons l'émergence de la catégorie socio-économique des "nouveaux ouzbeks », ce qui nous permettra de souligner les paradoxes en matière d'investissement de l'argent rapporté par la migration. Il s'agit finalement de montrer que les migrants, au centre du rite de transition dont ils sont les acteurs, se désengagent peu à peu lors du retour et se replient sur ce qu'ils considèrent être leur ouzbékité.

\section{BOULEVERSEMENT ET RÉAMÉNAGEMENT DES STATUTS AU SEIN DE LA PARENTÉ : LES EFFETS SOCIAUX DU RITE DE TRANSITION}

Le départ et surtout l'absence du migrant ont des conséquences directes sur la famille restée au pays d'origine. On assiste à une paradoxale occidentalisation des proches qui, depuis l'Ouzbékistan, cherchent à travers les médias occidentaux de nouveaux modèles de vie. Les valeurs et fonctions familiales changent, la place du père, du frère ou de l'époux restant inexploitée en raison de la migration. De retour, le migrant devra retrouver une nouvelle place dans l'organisation domestique. 


\section{Vers une occidentalisation de la famille?}

La première conséquence visible de l'émigration est le faible pourcentage d'hommes dans certains lieux ${ }^{4}$. Samarkand est actuellement réputée pour être majoritairement habitée de femmes, d'enfants et de personnes âgées. Il s'agit sans aucun doute d'une exagération, mais cette impression souligne néanmoins une réalité tangible : de très nombreux hommes sont partis à l'étranger et leur absence pousse ceux qui restent à réorganiser le quotidien en fonction des places laissées vides. Il s'agit à la fois des postes laissés vacants ${ }^{5}$, mais aussi des places sociales : l'éloignement du père, du mari, du fils ou du frère n'est pas sans conséquence sur l'organisation domestique, sur le statut et les fonctions des différents membres de la parenté voire sur la définition même de la famille.

\section{Vers une parentalisation des grands-parents}

Dans bien des cas, les épouses des migrants s'installent avec leurs enfants chez leurs beaux-parents. Les parents des migrants accueillent leur bru et leurs petits-enfants dans la maison familiale et exercent l'autorité sur l'ensemble des habitants du logement. Cet état de fait s'explique par l'usage en Ouzbékistan de la résidence virilocale. Après le mariage, les époux gagnent généralement le domicile des parents du marié, avant de s'installer dans un logement indépendant. Seul le plus jeune fils reste traditionnellement chez ses parents jusqu'à leur décès. Mais dans le cas des migrations, c'est à nouveau chez ses beaux-parents que se retrouve bien souvent l'épouse du migrant. Elle y est soumise à l'autorité tutélaire de son beau-père, mais surtout à celle de sa belle-mère qui régente les activités domestiques. L'épouse se retrouve donc seule avec ses beaux-parents et ne peut compter sur son époux pour pacifier les relations. Elle n'a que peu de pouvoir décisionnel, hormis peut-être sur ses enfants, bien que ce ne soit pas toujours le cas, les grands-parents ayant leur mot à dire quant à l'éducation de leurs petits-enfants. Ils sont parfois amenés à prendre celle-ci totalement en charge. Il arrive en effet que des couples gagnent ensemble l'étranger, laissant leurs enfants chez leurs grands-parents en Ouzbékistan. C'est le cas de Farangiz, à Boukhara, qui héberge ses trois petits-enfants :

«Mon fils cadet est parti à Séoul il y a maintenant quatre ans. Au début, il était tout seul là-bas, mais il a voulu que ma bru vienne le rejoindre. Elle est partie là-bas depuis six mois. Leurs trois enfants sont restés ici avec nous, car c'est compliqué de partir avec eux. Et puis, il faut qu'ils grandissent ici ».

4 « Expert on labour migration from the Centre for Socioeconomic Research Lyudmila Maksakova believes that from 500000 to 800000 people of Uzbekistan's 27.6 million people are currently working abroad. However, this figure is not final, because in many Uzbek regions, especially in rural areas, adult members in almost every family leave for neighbouring countries to do seasonal jobs every year, leaving the elderly, women and children at home. At least one adult from other families works in foreign countries. ", http://www.uznews.net/news_single.php?lng=en\&cid=8\&nid=307, consulté le 17/07/2008.

5 Quatre migrants ouzbeks en Russie racontent au journaliste une des conséquences de l'émigration en Ouzbékistan : «À Geshvodan, notre village dans le sud de l'Ouzbékistan, il ne reste plus un seul homme. Tous partis en Russie! Cela pose un problème pour enterrer les morts, car la religion musulmane impose que ce soient les hommes qui posent les corps dans les tombes. La situation est devenue grotesque », http://www.bastamag.org/journal/article.php3?id_article=175, consulté le 17/07/2008. 
En effet, les migrants économiques n'emmènent pas leurs enfants à l'étranger, l'investissement que représenterait leur départ ne pouvant s'avérer lucratif alors qu'ils ne sont pas en âge de travailler. De plus, les migrants qui font l'objet de mes recherches n'ont pas vocation à s'installer durablement dans le pays d'accueil, et laissent donc en Ouzbékistan leur famille. Les grands-parents sont non seulement amenés à héberger leurs petits-enfants, parfois à les éduquer et s'en occuper au quotidien, mais également, pour certains, à les assumer financièrement. Certains migrants, et particulièrement ceux qui sont partis pour la Russie, ne donnent parfois aucune nouvelle pendant des années. Ce phénomène est intéressant, car il remet en question la place des parents comme éducateurs et perturbe la notion de foyer, où deux générations cohabitent, en l'absence de celle des parents. Ce bouleversement va de pair avec un changement dans le comportement des enfants.

\section{Changement de statut des enfants}

Les enfants en Ouzbékistan se trouvent en position inférieure par rapport aux aînés. Selon Poujol : "la première question posée lors d'une rencontre entre deux individus qui ne se connaissent pas concerne leur année de naissance respective afin de déterminer qui a l'ascendant sur l'autre » (2006 : 11). Le respect se traduit entre autres par un vouvoiement dû aux personnes plus âgées, même si la différence d'âge est infime. On peut aussi lire dans les termes d'adresses cette insistance sur l'âge. Si un frère aîné est appelé $a k a$, un frère plus jeune est désigné par $u k a$. Pour une sœur plus âgée, il convient de dire opa, mais singil pour une sœur plus jeune. Ce respect des plus âgés présente cependant une particularité : une jeune mariée se doit de vouvoyer les enfants de la famille de son mari nés avant son mariage, et d'être tutoyée par eux. La nouvelle arrivée dans la famille a donc un statut inférieur au plus jeune des enfants de la parenté. Hormis cette exception, le respect des enfants envers les aînés, qu'ils soient ou non de leur famille, est considéré comme une clef de voûte de la culture ouzbèke. Le respect et l'obéissance au sein de la famille apparaissent comme une évidence pour mes interlocuteurs. Cependant, ils semblent quelque peu mis à mal par l'éloignement du migrant. En effet, certains notent une nouvelle attitude des enfants " sans père », qui seraient amenés à remettre en cause les principes d'éducation de leurs parents. C'est ce que déplore Gulnoza :

"Entre quatorze et dix-huit ans, certains jeunes sont bizarres. C'est vrai, ce n'est pas facile, leurs parents les voient comme des enfants alors qu'ils se sentent déjà adultes. Mais le problème, c'est quand ils sentent un déséquilibre dans la famille, alors là, c'est trop mauvais. Ils s'occupent en traînant dans la rue et en allant dans les vidéos-bars avec leurs copains. Parfois même, ils consomment de la drogue. Mais c'est parce qu'ils n'ont rien à faire pendant leurs vacances! Avant, les jeunes allaient travailler avec leurs pères, comme ça ils ne faisaient pas n'importe quoi et en plus ils apprenaient un vrai métier. Mais maintenant, tout le monde est parti à l'étranger et leurs mères pleurent d'être toutes seules et les vieux se plaignent de devoir tout faire à la maison... Et puis, les gamins, tu leur dis d'apprendre un métier pour devenir quelqu'un de bien, un vrai homme sérieux, mais qu'est-ce que tu peux leur dire quand ils te disent qu'il n'y a pas besoin de faire des études pour partir à Moscou, et qu'ils préfèrent aller travailler quelque temps ailleurs plutôt que de devenir professeur ici et de ne pas avoir de quoi nourrir ta famille, même acheter du pain?". 
Ainsi, un souffle de rébellion verrait le jour dans la nouvelle génération d'adolescents ouzbeks, qui refuse le carcan traditionnel familial au profit d'une vie « à l'occidentale », selon leur propre dire. Manger des hamburgers ou des hot-dogs, aller en discothèque, jouer aux jeux vidéos en ligne dans les cybercafés, fumer, regarder des films érotiques seraient autant d'actes signant le rejet de la culture ouzbèke, du respect de l'autorité familiale et l'attrait pour un monde occidental fantasmé, dont les États-Unis seraient la métonymie, et la Russie la fenêtre d'accès vers cet univers. Poujol livre cette analyse : " quant à la crise d'adolescence officiellement inexistante dans ces pays de forte soumission aux aînés, il n'est pas dit que son coût social ne rejaillisse pas sur les générations plus âgées, sous la forme passive de la toxicomanie ou de l'alcoolisme et sous celle violente, du choix de l'extrémisme politico-religieux» (2006 : 11). Il semble que la crise d'adolescence, qui n'était pas une réalité culturelle auparavant en Ouzbékistan, fasse son apparition dans certaines familles de migrants. L'autorité paternelle est mise à mal par l'absence du père. C'est ce que raconte Šahnoza à propos de son cousin, qui s'est avéré être son frère biologique :

«Mon oncle et ma tante [maternels] ont donné à ma mère une fille, car elle $n$ 'arrivait pas à avoir d'enfant. Mais après, ma mère a réussi à avoir des enfants, et elle a donné à sa sœur [la tante maternelle de Šahnoza] un de ses fils, car ma tante n'arrivait pas à avoir de garçon. Ça arrive souvent ici, et on n'en parle pas vraiment, mais ça ne pose pas de problème, normalement. Mais mon oncle est parti pendant cinq ans en Russie, sans donner de nouvelles. Et mon cousin a appris à ce moment-là qu'il n'était pas son fils, enfin ... pas son fils de sang, quoi. Quand mon oncle est revenu, on était tous très heureux de le voir vivant. Mais mon cousin n'a pas accepté tout ça, et a commencé à lui répondre, à le regarder dans les yeux.

$C$ 'est vraiment la honte pour mon oncle, c'est vraiment difficile. Bon, là, il y a cette histoire qu'il n'a pas très bien prise. Mais on voit de plus en plus de jeunes qui $n$ 'obéissent plus trop bien à leurs parents, parce qu'ils ne les ont pas beaucoup vus à la maison, et ne sont plus habitués à leur obéir ».

Émerge ainsi une nouvelle catégorie, celle des adolescents, qui est marquée par un certain refus des habitudes ouzbèkes et une réticence face à l'autorité des parents, et en particulier du migrant qui, quand il revient, fait figure d'étranger auprès de ses enfants.

\section{Nouvelle place des femmes}

Le Comité pour l'élimination de la discrimination à l'égard des femmes des Nations Unies a montré du doigt l'Ouzbékistan, où « le poids des traditions et de l'idéologie patriarcale [constitue un] frein à l'émancipation des femmes $»^{6}$. Répondant aux experts, qui soulignaient les différences professionnelles entre hommes et femmes en Ouzbékistan, la délégation ouzbékistanaise a rétorqué que «dans les pays orientaux, une telle opposition entre la place des femmes dans la société, et celle des femmes dans la famille n'a pas lieu d'être. La femme a une fonction extrêmement sociale, qui est d'élever

6 Comité pour l'élimination de la discrimination à l'égard des femmes, « Le poids des traditions et de l'idéologie patriarcale en Ouzbékistan, frein à l'émancipation des femmes, inquiète fortement les experts », 10/08/2006, http://www.un.org/News/fr-press/docs/2006/FEM1574.doc.htm, consulté le 17/07/2008. 
ses enfants. Elle n'est pas prise en otage par sa famille, mais elle est mère, et membre actif de la société. Il s'agit avant tout d'un mode de vie ${ }^{7}$. Cet échange officiel apporte un éclairage sur la place de la femme en Ouzbékistan : la principale demande sociale qui lui est adressée est de mettre au monde des enfants, de les élever, de s'occuper des tâches domestiques et de ne pas porter atteinte à l'honneur de sa famille. Cela reste le cas aujourd'hui, néanmoins, un des changements majeurs dans l'organisation familiale est sans doute la nouvelle place occupée par certaines femmes dans la société. Cela se traduit par des actes qu'elles réalisent désormais, en l'absence des hommes, mari ou père, partis à l'étranger. C'est ce que résume Imrona :

"Maintenant, à Samarkand, on peut voir beaucoup de femmes dans les cafés et les restaurants. Des tablées de femmes, sans homme! Elles boivent même de la vodka, et tout! De toute façon, il ne reste plus que des femmes et des enfants, ici.

Tous les hommes sont partis gagner de l'argent ailleurs. Alors elles s'occupent. Elles ne peuvent pas attendre que les hommes reviennent pour vivre, c'est normal qu'elles sortent. Mais avant, cela ne se passait pas comme ça. C'est les hommes seulement qui sortaient aux restaurants. Parfois ils emmènent leur épouse, mais elle ne sort pas dans des lieux comme ça toute seule, ce n'est pas comme en France! ».

Les femmes des migrants auraient donc fait évoluer les mœurs féminines, vers un déplacement de la sphère domestique à la sphère publique. Cette dernière, généralement dirigée par les hommes, est investie par des femmes qui ne peuvent plus compter sur leurs époux, absents, pour organiser des sorties dans les lieux publics. De la même façon, certaines femmes de migrants décident de conduire une voiture personnelle.

"Avec l'argent envoyé par Ališer, on a acheté une voiture, une Nexia [de la marque Daewoo]. Mon fils l'utilise parfois, mais il n'est pas là souvent. Alors j'ai décidé de me mettre à conduire. J'ai pris quelques cours et ça va. Enfin..., j'ai peur quand même! [rires]. Mais c'est mieux pour moi, autrement je n'irais plus dans notre datcha. Et puis les transports en commun où on est serré, ce n'est pas pour moi. Les taxis, oui, mais autant avoir sa voiture particulière " [Nodira].

Passer son permis de conduire, décider de l'orientation du budget familial, exercer un métier prenant ou encore aller dans un café sont autant d'actions, considérées a priori comme réservées aux hommes, que des femmes de migrants font pourtant. En l'absence de l'autorité masculine, elles sont amenées à prendre des décisions qui reviendraient ordinairement à leur époux.

\section{Remise en question de l'autorité masculine}

Les migrants qui posent le pied sur la terre d'origine éprouvent des sentiments contradictoires : au soulagement et au plaisir de retrouver tout ce qui a manqué pendant l'exil se mêle une sensation de dépossession de ce qui les entoure. La place des hommes, puisque ce sont eux qui émigrent en majorité, a été transformée pendant la migration.

7 Ibid.

REMI 2010 (26) 3 pp. 59-84 


\section{L'étonnement du migrant devant les changements}

Les migrants qui reviennent sont d'abord surpris des changements au sein de leur famille, et des nouveautés architecturales de leur lieu d'origine. Beaucoup disent leur étonnement lors des retrouvailles avec leurs enfants grandis ou d'autres parents et ces changements leur font prendre conscience du temps passé en migration. Les travaux effectués dans la ville ou le village d'origine les surprennent aussi. Enfin, certains notent avec stupeur la raréfaction des hommes en Ouzbékistan, " tous partis à l'étranger », comme ils le disent eux-mêmes. Pour Ziëd, alors qu'il me présente une des enseignantes de physique-chimie de l'université de Namangan :

"Quand je suis parti, elle était mon étudiante. Maintenant, je suis revenu et elle est ma collègue! [rires]. C'est idiot, mais ça m'a fait très bizarre à la rentrée de discuter des cours avec elle. Je me suis dit "que de chemin parcouru!" Et j'étais en Corée pendant tout ce temps-là. C'est la vie! [en français]».

Le « chemin parcouru », c'est à la fois celui du migrant à Séoul et celui, parallèle, de son ancienne étudiante à Namangan. Mais ce qui marque particulièrement les migrants, qui l'expriment à demi-mot ou par le biais de l'humour, c'est le sentiment d'une perte de valeurs dans la famille d'origine, et d'un déclassement de leur autorité. Ils le craignaient parfois déjà depuis l'étranger, comme Ališer, rencontré à New York :

"Je ne comprends pas très bien l'évolution des choses, des fois. Je vois bien que mes enfants sont très attachés au fait d'avoir des marques de vêtements à la mode.

Ils n'en ont jamais assez! Je ne sais pas combien il faut avoir de pantalons pour être à la mode, mais il faudra bientôt acheter une maison pour pouvoir ranger leurs affaires, je pense! [rires]. À chaque fois que je téléphone, j'apprends qu'ils sont allés faire les magasins. Mais moi, dans mon village, je n'avais qu'un seul change, et je ne me posais pas de questions sur ça. Aujourd'hui, pour les jeunes, c'est très différent. Ma femme m'a dit que ma fille, il n'est pas question qu'elle porte une robe ouzbèke [ici, renvoie aux robes fabriquées sur mesure à partir d'un coupon de tissu]. Il faut absolument qu'elle aille acheter un vêtement chinois, ou turc, ou je ne sais pas quoi. Mais quand même, c'est nos traditions, il faut garder ça ».

Ce besoin des enfants d'obtenir des produits dont ne disposaient pas leurs parents dans leur enfance revient souvent dans le discours des migrants, qu'ils soient encore à l'étranger ou déjà revenus. Vêtements à la mode, mais aussi appareils électroniques sont devenus l'apanage des enfants des migrants, qui cherchent à se démarquer des générations précédentes en s'occidentalisant, en se rapprochant des cultures russes et américaines, par les habits ou la musique.

Poujol note l'apparition "de comportements "d'enfants gâtés" dans les milieux urbains favorisés du Kazakhstan, s'apparentant à "l'enfant-roi” de la société chinoise en plein développement et que l'on peut rapprocher du contexte ouzbek. Ainsi, autant l'amélioration des conditions de vie pour une fraction réduite de la population ajoutée à l'ouverture brutale des médias sur le monde extérieur (accès à Internet, parabole, diffusion des films étrangers) ont pu avoir des conséquences psychologiques décelables sous forme de pathologies connues en Occident (addiction aux jeux vidéo, crises de délire), la préca- 
risation brutale d'une part croissante de la population a nécessairement une traduction psychiatrique » (2006: 12).

Dans le cas des migrations, il y a bien la découverte par le migrant d'une crise familiale qui s'est produite à son insu, et qui entraîne une exigence d'un apport financier régulier, les dépenses générées par la famille étant désormais supérieures à celles précédant le départ.

\section{Changement du statut de père, de fils et d'époux}

Les migrants reviennent donc dans une famille où les enfants ont grandi, où l'épouse a pris une nouvelle place dans la sphère publique et où les parents assurent un rôle domestique nouveau. Ces changements de mœurs se sont opérés progressivement, mais sans que les migrants ne les perçoivent nécessairement depuis l'étranger. Il se retrouve donc face à une situation qui les isole. Cela engendre un décalage dans le rapport du migrant à sa famille :

" J'étais vraiment pressé de rentrer en Ouzbékistan. J'y pensais tout le temps à Séoul, je m'imaginais comment ce serait de se retrouver chez soi. Mais finalement,

ce n'est pas aussi simple que je le pensais. Bien sûr, je suis content d'être là, ce n'est pas ça. Mais les choses ont changé un peu pour moi. Je n'accepte plus comme avant que mes parents gèrent ma vie, me disent de faire ci ou ça avec mon argent. Et puis je pense qu'eux aussi ne me disent rien, mais ils trouvent que j'ai changé. Ils m'appellent parfois "le Coréen", pour rire. Je ne sais pas ce qui se passe exactement, mais je n'ai plus envie de travailler pareil que là-bas, et en même temps, ça me manque un peu. Car là-bas, je ne me posais pas autant de questions, je faisais ce que j'avais à faire, c'est tout » [Ilhom].

Plusieurs éléments sont à retenir dans ce récit et tout d'abord le sentiment de désœuvrement consécutif au retour. Les migrants ne retrouvent pas nécessairement une activité professionnelle à leur arrivée en Ouzbékistan, et beaucoup s'accordent une période de repos. Le rythme de travail jugé éreintant dans le pays d'accueil est évoqué avec regret par certains migrants qui se sentent désormais inutiles et sans objectifs. En migration il s'agissait de travailler pour amasser de l'argent et revenir au pays, une fois ce stade atteint les incitations à travailler sont beaucoup moins fortes. Ilhom note aussi son changement d'attitude face à ses parents. Il n'accepte plus les remarques qui lui sont adressées et le manque de liberté qu'il éprouve est nouveau. L'éloignement lui a fait oublier l'autorité parentale omniprésente et omnipotente, qu'il rechigne désormais à respecter. Pour Poujol : «La fin du système soviétique a produit une rupture éthique et comportementale due à la perte brutale des repères dans des sociétés auparavant fortement structurées. Cette rupture ne s'est pas accompagnée d'explications ni de dialogues entre les générations, parents-enfants notamment. Le non-dit a été la règle, à part quelques commentaires au sein des classes d'âge qui ont un grand rôle de structuration sociale en Asie centrale. À ceci ont succédé assez rapidement des considérations nostalgiques de la part des générations de retraités qui constatent de façon récurrente : "avant, cela ne se passait pas comme ça...” $"(2006: 12)$. 
Ce changement de comportement consécutif à la migration est même craint par certains parents, qui rechignent à laisser partir leur enfant à l'étranger de peur que cette expérience ne le fasse évoluer négativement. Ainsi pour Nodira :

"Tous mes enfants parlent anglais, et ma deuxième fille a eu l'occasion de partir aux États-Unis. Mais je n'ai pas voulu. Elle m'en veut à cause de ça. Mais d'abord, je ne voulais pas qu'elle prenne l'avion, c'est trop dangereux. Et puis quand même, je connais ma fille, mais on ne sait jamais ce qu'il peut arriver là-bas, elle peut changer de comportement. Je veux que mes enfants continuent à être bien élevés, à baisser la tête et à obéir à leurs parents, même s'ils sont adultes. Et à ne pas regarder leur père dans les yeux. Ceux qui partent, on ne les reconnaît plus quand ils rentrent, ils sont devenus effrontés, sans respect. »

À ce generation gap, pour reprendre le terme anglo-saxon, entre le migrant et ses propres parents, s'ajoute celui entre le migrant et ses propres enfants, qui eux-mêmes ne s'inclinent plus devant l'autorité du migrant. Dès lors, quelle place peut occuper le migrant au sein de sa famille?

\section{Se taire et s'individualiser}

Le migrant s'est bien souvent construit une image de l'Ouzbékistan basée essentiellement sur une nostalgie de la patrie quittée et de l'entourage familial regretté. Il a également élaboré un investissement symbolique important quant à une tradition ouzbèke, que l'on a qualifié d'ouzbékité, qui reposerait sur des valeurs morales opposées à la «nonculture » des pays d'accueil. Or inversement, les membres de sa famille ont souhaité profiter des capitaux étrangers pour s'occidentaliser à la manière ouzbèke, s'il est possible de le décrire ainsi. Les femmes sont devenues plus indépendantes en investissant la sphère publique, en choisissant des biens de consommation visibles par tous, en opérant des actes jugés virils, tels que celui de conduire ou de s'arrêter à une terrasse de café. Les enfants des migrants se sont tournés vers ce qu'ils estiment être une culture américaine ou européenne, en choisissant des vêtements, de la nourriture et des attitudes qu'ils jugent à la mode.

Tout le paradoxe de la migration est là : l'argent amassé en migration sert, en Ouzbékistan, à imiter un style de vie étranger, que n'a pourtant pas adopté le migrant dans le pays d'accueil, puisque celui-ci s'est au contraire tourné vers la culture du pays d'origine. Les migrants n'évoquent que très peu leur expérience migratoire. Peu de précisions sont apportées sur la façon dont ils ont vécu la migration. À ce sujet on peut se référer à Temine (1999: 136) qui mentionne les « dix commandements destinés [...] à faciliter l'insertion des travailleurs immigrés dans la société française », parus en janvier 1899 dans le journal Emigrato. Le dixième commandement dit : «de retour au pays, ne dis pas de mal de la terre qui t'a nourri, ni des hommes qui ont vécu avec toi ».

De la même façon, suivant ce précepte tacite, les migrants n'évoquent presque jamais leur travail en migration. Les familles ne posent d'ailleurs pas de questions sur les conditions matérielles dans le pays d'accueil. Le migrant constate donc à la fois combien son absence a conduit à des remaniements familiaux importants, combien la période 
migratoire a été vécue différemment par ses proches et par lui-même et, parallèlement, il n'évoque qu'à demi-mot cette constatation souvent mêlée de dépit. Pour retrouver une nouvelle place dans la société, il lui faut mettre en avant son enrichissement, afin que son statut d'homme « qui vit bien » soit admis par tous, sans pour autant dévoiler les moyens dont il a usé pour parvenir à cette fin. Il est devenu " nouvel Ouzbek », et une nouvelle charge sociale lui incombe désormais : celle de faire fructifier ses réseaux nouvellement acquis.

\section{Émergence des « nouveaux Ouzbeks » : la ligne de séparation créée par le rite d'institution}

À Séoul, après avoir fait étalage de ses deux téléphones portables, de ses mails qu'il dit consulter tous les jours, du restaurant ouzbek de Paris qu'il a découvert sur Internet, de son amour pour les beaux vêtements et de son rêve de voir un jour la Tour Eiffel, Odil me demande d'estimer son âge et, juste après, veut savoir s'il ressemble à un Ouzbek... Que répondre et que veut-il entendre ? Qu'il ne ressemble pas à un Ouzbek car la migration l'a changé en occidental?

Comme le note Centlivres (2000: 5), dans les sociétés à cheval entre plusieurs États, « le processus identitaire déborde également le choix entre deux identités nationales. Bref, diasporas, migrations et collectivités "transmigrantes" sont des figures d'une nouvelle normalité du fonctionnement des sociétés modernes ». On peut alors se demander comment cela se traduit pour les migrants ouzbeks de retour dans le giron familial.

\section{Historique de cette catégorie officieuse}

Au cours des entretiens réalisés, tant dans les pays d'accueil que dans le pays d'origine, j'ai pu constater qu'il n'existait pas de terme général pour désigner les migrants. J'utilisais pour ma part, en russe, celui de migranty, qui était compris par mes interlocuteurs, mais rarement repris à leur compte. Les migrants ne se désignent pas en tant que tel, ne se représentent pas comme groupe collectif car ils ne veulent pas exister en tant que « migrants ». Ils se définissent davantage comme Ouzbeks quand ils sont dans le pays d'accueil. Mais comment désigner ceux qui sont rentrés?

En réalité, la migration apparaît comme une phase d'entre-deux, où les migrants sont en transition vers un nouvel état : ils ne relèvent déjà plus vraiment de leur ancien statut qui serait celui d'Ouzbeks en recherche de mieux-vivre, et pas encore intégrés dans celui qu'il recherche, c'est-à-dire celui d'Ouzbeks enrichis revenus dans leur pays d'origine. Comment appeler ces anciens migrants rentrés en Ouzbékistan avec un changement visible de mode de vie et de consommation ? Un terme semble progressivement s'imposer : celui de «nouvel Ouzbek ». Il est sans aucun doute un dérivé de l'expression russe novyj russkij [r. : litt., nouveau Russe], qui avait lui-même repris l'expression russe novorič dérivée du français « nouveau riche ». En russe, ces deux expressions renvoient aux entrepreneurs et aux oligarques qui se sont brusquement enrichis à la chute de l'URSS, souvent par le biais d'actions illégales et en partenariat avec des réseaux mafieux. 
En Ouzbékistan, l'expression « nouvel Ouzbek » évoque surtout l'idée d'un parvenu, quelqu'un ayant subitement changé de statut financier et qui entend le faire savoir autour de lui. Cette expression est employée pour tous les cas d'enrichissement rapide. Elle ne relève pas nécessairement de l'ordre du compliment, bien que beaucoup envient cette position. Absence de discernement dans les dépenses et faste déployé dans le seul but d'impressionner l'entourage sont souvent critiqués par les témoins de ce changement. C'est ce que note avec ironie Nilufar :

«Notre voisin, je l'ai connu tout petit. Il jouait souvent avec mes enfants. Il $m$ 'appelait tata, on était proche. Il aimait bien rester manger à la maison, il était simple. Mais depuis qu'il est revenu de Corée, il se prend pour l'émir [rires] ! Il ne peut pas sortir sans son téléphone portable et, quand il arrive devant chez ses parents, il klaxonne soi-disant pour qu'on lui ouvre le portail, mais moi je crois plutôt que c'est pour montrer à tout le monde sa nouvelle Nexia ».

Le changement financier est donc noté, mais accompagné d'une critique sévère du changement d'attitude. Comme l'explique un article de presse, l'Ouzbékistan est « une immense lessiveuse des profits illicites de toute la région. Seule une très petite partie de l'argent blanchi est investie sur place, mais il alimente des importations de produits de luxe : parfumerie française, vêtements de luxe et supermarchés d'alimentation hors de portée de l'immense majorité de la population. La force de ce dispositif, outre qu'il favorise l'émergence d'une classe relativement aisée, embauchée dans la nébuleuse des entreprises mafieuses, c'est qu'il s'enracine profondément dans la structure sociale du pays $\gg^{8}$. Les nouveaux Ouzbeks en sont les acteurs. Ainsi, le gendre d'Ulugbek et de Maftuna, à Tashkent, est-il prêt à dépenser l'équivalent de plusieurs salaires moyens en Ouzbékistan pour s'acheter des vêtements de manufacture européenne. Il s'agit d'avoir de l'argent et, surtout, de le faire savoir. Pour cela, il convient de se montrer à la fois généreux et de placer ses investissements dans des activités visibles.

\section{Un classement par l'argent}

Un classement social semble établi par l'argent, en Ouzbékistan, sans que soient mis en question les moyens d'acquisition de cette soudaine fortune. Comme le rappelle Amandine Regamey, qui analyse les histoires drôles, « le nouveau russe est un "nouveau riche” caractérisé par sa bêtise, son mauvais goût, mais ses énormes possibilités financières (acquises illégalement) qui lui permettent de tout acheter, femmes et policiers compris » (Regamey, 1999 : 5). De la même façon, un nouvel Ouzbek est caractérisé par son ascension financière fulgurante, mais dont l'origine est inconnue. Dans le cadre de la migration économique, il ne s'agit pas nécessairement de capitaux acquis via des réseaux mafieux, mais souvent illégalement, les migrants ayant pour beaucoup séjourné sans autorisation dans le pays d'accueil et n'ayant pas été déclarés par leurs employeurs. Par ailleurs, comme ils évoquent de manière très lacunaire leurs conditions de vie à l'étranger, celle-ci apparaît floue et auréolée de mystère.

8 http://www.voltairenet.org/article7396.html, consulté le 20/07/2008. 
C'est ce que soulignent aussi Bennegadi et Bourdillon (1990 : 131) à propos des migrants maghrébins en France : «on note souvent quelques difficultés pour le travailleur migrant qui revient [...] dans son groupe d'origine, à expliciter clairement le travail qu'il fait. Lorsque l'écart est trop grand symboliquement entre le statut social de la personne dans son groupe d'origine et celui dans le pays d'accueil, se développe toute une stratégie de non-dit où la complicité est totale, admise et opérante; on ne parle que du côté gratifiant de ce travail sans avoir à dire en quoi il consiste ».

L'important pour le nouvel Ouzbek est d'avoir accumulé une somme d'argent importante lui permettant de montrer à qui veut le voir son nouveau statut social. Cette ascension est étonnamment dépouillée de valeurs morales. Ainsi, si des habitants suspectent parfois certaines voisines d'avoir gagné l'Arabie saoudite pour s'y prostituer, et revenir dans leur pays d'origine avec des moyens nouveaux, ils considéreront néanmoins cet argent comme une marque de changement de statut. L'échelle de valeur n'est pas basée sur les valeurs morales, mais uniquement financières.

Il ne s'agit pas nécessairement d'une remarque positive. Pétric (2006 : 213-214) évoque le cas des entrepreneurs enrichis à la faveur de la perestroïka et explique qu' « il s'agit en général d'individus n'ayant pas fait d'études. [...] Ces individus, ayant fait parfois fortune très rapidement, bénéficient d'une grande autorité sociale grâce à leur carrière. [...] Les plus riches d'entre eux sont appelés yangi Uzbeklar ou novi Uzbeki (nouveaux Ouzbeks), en référence à l'expression "nouveau riche”, du fait de leur ascension sociale très rapide et de leur niveau culturel médiocre ». Être nouvel Ouzbek, c'est éprouver le besoin de montrer son argent, et cet étalage provoque à la fois des regards envieux, mais aussi des moqueries. C'est ce que m'explique Nodir qui évoque le cas de son oncle :

"Mon oncle, c'est vraiment mon modèle. Il est parti avec très peu d'argent, au départ. Mais il a fait du business, et comme c'est un très bon businessman, il a commencé à très bien gagner sa vie. Je voudrais être comme lui. Enfin, non, pas tout à fait, parce qu'il est vraiment devenu trop riche, et moi, je ne veux pas être un nouvel Ouzbek [rires]. Je ne veux pas que l'argent me monte à la tête, et sortir en cravate, les mains derrière le dos [rires]. Je suis trop jeune pour ça. Mais gagner de l'argent, oui. Et avoir de l'autorité [Il le dit en se tapant sur le ventre], car pour l'instant, ce n'est pas encore ça [rires] ».

Avoir de l'argent, c'est pouvoir se permettre d'adopter certaines attitudes, telles que « porter la cravate » ou « avoir les mains dans le dos », position des hommes respectables qui marchent d'un air assuré, ou encore « avoir de l'autorité », qui se matérialise par un ventre proéminent, signe que l'on a les moyens de beaucoup manger.

\section{Changement de réseaux sociaux et nouvelle vision politique}

Mais qu'apporte exactement ce changement de situation financière ? Que cache cette opulence exhibée à grand renfort d'ostentation? Sans doute la motivation première des migrants est-elle l'amélioration des conditions matérielles de leur famille. Mais comme le soulignent Adelkhah et Olszewska (2006) à propos des Afghans, la mobilité peut être envisagée « comme une stratégie sociale ». Avoir de l'argent, ce n'est pas unique- 
ment pouvoir en faire profiter ses proches, mais également nouer de nouvelles alliances, agrandir le cercle social des interdépendances.

Ainsi, un migrant enrichi et revenu dans le pays d'origine, pourra-t-il, par exemple financer le départ de nouveaux migrants, ce qui rendra ceux-là ainsi que leur famille redevables. De la même façon, les cadeaux qu'il apportera lors des différentes cérémonies festives, telles que les mariages et les circoncisions, ainsi que la magnificence avec laquelle lui-même organisera ces rassemblements, pousseront ses hôtes à l'inviter dans d'autres circonstances, et à renforcer les liens ; connaître un homme fortuné étant de bon aloi.

Ces relations sont principalement établies au regard de la position financière de l'ex-migrant, et non de son charisme, de sa loyauté, de sa sagesse ou surtout de son âge, qui sont pourtant des caractéristiques prépondérantes des figures de l'autorité en Ouzbékistan. Ici, il s'agit de connaître des hommes fortunés et de se faire reconnaître comme riche. Plus un homme est riche, plus il est amené à dépenser de l'argent pour les autres, dans un système de dépenses en escalade. Il faut donner toujours plus que l'autre, organiser des fêtes encore plus somptueuses, se montrer encore plus généreux dans les dons. Ceci n'est pas sans rappeler le potlatch décrit par Mauss (2001), ce système de dons et de contre-dons sur un mode antagoniste, les bienfaiteurs cherchant sans cesse à aller plus loin que l'autre dans l'offre.

Il est habituel d'entendre dire, en Ouzbékistan, que la richesse d'un homme se mesure davantage à son réseau social qu'à son portefeuille. Le paradoxe des Nouveaux Ouzbeks réside dans le fait que c'est justement leur argent qui leur a permis d'étendre et de consolider leur réseau social.

\section{Les célébrations rituelles pour éviter la chute}

Les migrants ont acquis, par l'argent ramené des pays d'accueil, une nouvelle façon de marquer leur espace social. Leur nouveau statut de « nouvel Ouzbek » est pourtant précaire : en cas de paupérisation, ces relations superficielles s'effaceront. C'est en tout cas ce que craignent les migrants. Dès lors, des protections symboliques sont recherchées pour éviter la chute, prévenir la déchéance.

J'ai pu assister à différents rites et les migrants m'en ont fait découvrir d'autres. Le plus commun est le is ou hatimkoran, c'est-à-dire littéralement « lecture du Coran ». Un animal, poulet, mouton ou bœuf, est sacrifié, le sang servant à marquer l'endroit qu'il convient de protéger. Un mollah récite une prière. La cérémonie se conclut par un repas, où l'animal sacrifié est cuisiné et partagé par les convives. Il convient pour les migrants de chercher une garantie symbolique de conserver leur statut acquis par la migration. Il s'agit surtout de se prémunir du mauvais œil. En effet, plus l'on est regardé par les autres, plus on est admiré, plus le risque est grand de perdre ce que l'on a, de tomber malade, de redescendre l'échelle sociale. Les migrants enrichis attirent des regards envieux, dont il faut se prémunir pour éviter la chute. 
Des réunions féminines sont organisées par les épouses des migrants ${ }^{9}$, dans ce même but, tels que les muchkulkuchod ou bibisechanbe. Ce sont des cérémonies religieuses menées par une bibihalfa, femme pieuse qui équivaut plus ou moins au mollah pour les femmes. Les participantes et surtout l'organisatrice espèrent être ainsi protégées des aléas de l'existence.

Tous ces éléments religieux doivent permettre d'éviter ce que les migrants craignent le plus : la chute, à la fois sociale et financière. Par ailleurs, les investissements de l'argent de la migration sont effectués dans l'espoir de se maintenir dans une situation économique confortable. Mais les exemples des migrants malheureux continuent à les inquiéter.

\section{Significations et enseignements des échecs migratoires}

Il convient de revenir ici sur les cas d'échecs au cours des migrations. Dans les rites de passage, les candidats ne passent pas tous l'épreuve avec réussite. Certains ne parviennent pas au terme de l'initiation, notamment quand le rite est particulièrement éprouvant pour le corps, et cet échec peut se traduire par le décès. En migration, il n'y a évidemment pas de mutilations ou autre marquage du corps ritualisé. Cependant, l'affaiblissement et l'amaigrissement peuvent être considérés comme des traces visibles du passage en migration. Les marques physiques (cicatrices, membres amputés, etc.) résultant d'agressions racistes en Russie en font aussi partie, comme me l'explique une étudiante tadjike de Samarkand rencontrée à Paris. Elle me demande ainsi de me rendre chez ses parents et d'essayer de faire parler son père pour connaître son histoire migratoire restée secrète :

"Tu sais, avec mon père, on ne sait pas vraiment ce qu'il s'est passé. Il est parti pour travailler en Russie. Et puis, on n'a pas eu de nouvelles. Ma mère était très inquiète, nous [les enfants du couple], on ne comprenait pas très bien ce qu'il se passait. On ne recevait pas du tout d'argent, mes grands-parents ont dû nous aider

à vivre. On a essayé de le retrouver, en interrogeant des gens qui étaient allés à

Moscou et auraient pu le rencontrer. On a même fait une émission de télé pour lui demander de revenir, on a pleuré, c'était dur. On a attendu, attendu. Finalement, on a pensé qu'il était mort, et on a réorganisé notre vie sans lui.

Un soir, il a frappé à la porte. Il est entré. Il lui manquait des doigts à la main. Il n'a jamais rien expliqué, on ne sait absolument rien. Ma mère a décidé de ne pas lui poser de questions. Moi, j'ai essayé, mais alors les larmes lui montent aux yeux et il ne répond pas. Son retour, ça a été une grande joie pour nous, de voir qu'il était bien vivant, mais après, il y a eu des problèmes. Surtout avec mon frère, qui a appris entre temps qu'il était en réalité le fils de ma tante, et que ma cousine est en fait ma sœur biologique. Du coup, il ne supporte plus l'autorité de mon père, car il s'est senti abandonné. C'est très dur pour mon père de ne pas être respecté par son propre fils... enfin, qu'il considère comme son fils. Surtout qu'en Ouzbékistan, tu le sais, c'est très important.

9 «En Asie centrale, les hommes et les femmes ne pratiquent pas de la même manière et ont ainsi un rapport au religieux différent. [...] Le nombre d'édifices religieux conçus pour les femmes et destinés à leur offrir une éducation religieuse est considérablement inférieur à celui conçu pour les hommes » (Fathi, $2004: 239-240$ ). 
Moi, je comprends qu'il a dû vivre des moments très difficiles, et qu'il ne veut pas nous les raconter. Mais toi, tu es étrangère, et tu travailles sur les migrations, peut-être qu'il pourra t'en parler et que tu me diras ce qu'il s'est vraiment passé? 》.

L'émission russe « Ždi menâ. Nacional'naâ služba vzaimogo poiska lûdej » ${ }^{10}$ [Attends moi. Service national de recherche mutuelle de personnes] est un moyen souvent utilisé par des familles centrasiatiques dans l'espoir de retrouver un parent parti travailler en Russie. Certaines familles restent ainsi sans recevoir aucune nouvelle, ou encore apprennent le décès de leur proche, sans plus de détails sur les circonstances de sa mort ${ }^{11}$. Cependant, ces problèmes liés à la migration ne sont pas évoqués clairement par les familles restées en Ouzbékistan, car à l'inquiétude due à ce silence s'ajoute la honte de ce qui est considéré comme un échec et, parfois, la peur de ne pouvoir rembourser les dettes contractées pour le départ.

Face à ce silence, la plupart des gens n'insistent pas et ne posent pas plus de questions. Mais mon passage en Ouzbékistan a été l'occasion pour certains de lever une partie du voile opaque qui empêche de savoir ce qui se passe « vraiment » à l'étranger. En effet, à la suite de mes trois terrains dans les pays d'accueil, je suis donc repartie en Ouzbékistan. Les questions concernant la migration pouvaient m'être adressées plus facilement en ma qualité d'hôte étrangère, donc non impliquée dans les mêmes logiques socioculturelles qu'un migrant ouzbek, mais ayant vu différentes situations migratoires de mes propres yeux. Répondre à ces interrogations générales ne me posait pas trop de problèmes, bien que cela rende le chercheur démiurge de son propre sujet. Cependant, l'engagement était bien différent quand les questions portaient sur un migrant spécifique. Comment répondre aux familles, ne sachant pas ce que le migrant avait lui-même dévoilé ou non de sa vie à l'étranger?

Le problème s'est en réalité posé de manière moins aiguë que je ne le craignais, les familles préférant souvent être rassurées sur l'état de santé et le moral du migrant, plus que sur ses conditions exactes de vie à l'étranger. Mais il m'est arrivé en Ouzbékistan que des familles fassent venir une connaissance, un ami ou un voisin, revenue de l'étranger, pour que je puisse l'interroger devant eux, et en savoir ainsi davantage sur son parcours.

En définitive, l'échec de la migration peut se traduire par une absence de nouvelles due soit au décès, soit à l'impossibilité d'en donner, soit à la honte du migrant d'être dans une situation matérielle peu enviable. Cela peut nous faire songer à ce que décrivait Van Gennep (1981 : 108) : "le novice est considéré comme mort, et il reste mort pendant la durée du noviciat. Celui-ci dure un temps plus ou moins long et consiste en un affaiblissement corporel et mental du novice. [...] [Ensuite,] on le ressuscite et on lui apprend à vivre, mais autrement que pendant l'enfance ». Les initiés qui s'en sortent sont ensuite agrégés à un nouveau groupe à leur retour dans le pays d'origine.

10 Cf. http://poisk.vid.ru/, consulté le 20/07/2008.

11 «Focus on southern labour migration », http://www.uzland.info/2005/march/09/02.htm, consulté le 20/07/2008. 


\section{INVESTISSEMENT DE L'ARGENT ET BOULEVERSEMENTS ÉCONOMIQUES}

L'argent des migrations joue un rôle crucial dans l'économie du pays d'origine. Mais comment est-il investi concrètement et quel apport représente-t-il pour le pays ? L'une des principales dépenses est le mariage des enfants du migrant ou des migrants eux-mêmes, fête particulièrement onéreuse en Ouzbékistan. Ensuite, il s'agit du logement, l'achat immobilier représentant un investissement durable pour la famille. Il peut aussi être injecté dans des secteurs lucratifs, dans des projets potentiellement rémunérateurs. Enfin, il peut servir à acquérir des preuves de richesse, afin de démontrer au voisinage combien la migration a été bénéfique pour l'amélioration de la vie quotidienne.

\section{Mariages et autres célébrations}

Le mariage est célébré avec faste en Ouzbékistan et les festivités qui y sont liées sont particulièrement onéreuses. De nombreux Ouzbeks s'endettent pour parvenir à faire face à de telles dépenses. L'argent économisé au cours de la migration permet d'accueillir comme il se doit les invités.

\section{Importance et intérêt de la dépense}

En Ouzbékistan, comme dans bien d'autres pays, les passages de la vie sociale considérés comme les plus déterminants sont caractérisés par des regroupements festifs. Ils sont destinés à marquer ces transitions et à les effectuer en respectant ce que l'on peut rapidement nommer les traditions, bien que ce terme ne doive pas être compris comme relevant d'une histoire figée, mais au contraire comme une adaptation permanente entre les valeurs culturelles et les possibilités contemporaines de leur réalisation.

Plusieurs événements sont ainsi célébrés avec faste, différemment selon le sexe des individus. Ruffier (2002 : 52, 79) a analysé ces fêtes, qu'il qualifie dans le titre de son ouvrage d' " espaces de construction des identités et des solidarités » : "les Ouzbeks donnent comme prétexte à l'organisation d'une to'y l'accouchement du premier enfant tug'ich to'yi, la mise au berceau - beshik to'yi, la circoncision - sunnat to'yi, le mariage - nikoh to'yi, l'installation dans une nouvelle maison - hovli to'yi, un anniversaire important (cinquante ans, soixante-trois ans [âge de la mort du Prophète], soixante-dix ans) - jubile to'yi. [...] Les quatre étapes [principales] du cycle des to'y, circoncision/ mariage/circoncision/mariage, dans la vie d'un individu tout d'abord sujet de la célébration, puis organisateur, forment clairement un cycle d'insertion progressive dans une communauté de vécu ». On voit ici que cela est envisagé du point de vue masculin, avec la circoncision.

Le point d'orgue de la vie sociale d'un individu est sans doute son mariage, qui doit le faire définitivement passer au statut de père ou de mère de famille, et celui qu'il organisera pour ses enfants. La fête se déroule traditionnellement chez les parents de l'époux. Ils reçoivent les invités dans la cour de leur maison ou à l'extérieur de celle-ci ou encore, pour certains, dans un restaurant. Cette dernière possibilité est très prisée par 
les « nouveaux Ouzbeks » car il s'agit alors d'un mariage encore plus onéreux et visible par tous. Il y a différentes étapes dans le mariage et l'enregistrement administratif, appelé ZAGS, n'est que le prélude à une longue festivité. Bien que la famille du marié prenne en charge la réception principale, celle de la mariée est aussi fortement impliquée, par un jeu de dons et de contre-dons entre les deux parties.

Les sommes engagées dans la réception des invités d'un mariage sont très importantes et plusieurs Ouzbeks m'ont affirmé qu'un seul mariage représentait parfois jusqu'à une dizaine d'années d'économie pour un père. Ce sacrifice est consenti par de très nombreux parents, qui sortent parfois ruinés de la noce de leurs enfants, mais fiers. Aujourd'hui, certains migrants partent donc pour pouvoir réunir plus rapidement cette somme et ainsi marier dignement leurs enfants. Il s'agit d'une question d'honneur, et non, au sens propre, d'une tentative de lutte pour sa survie. À moins que l'on puisse considérer l'organisation d'un mariage comme une nécessité pour la survie sociale d'un parent, ce qui se rapproche assez bien de ce que j'ai pu observer. Jadis, il était d'usage que ce soit les parents qui assument seuls les dépenses liées au mariage. Actuellement, certains jeunes gens décident de soulager leurs parents et de partir à l'étranger pour réunir eux-mêmes la somme nécessaire à l'organisation d'un mariage « digne de ce nom ». Mais qu'est-ce qui se trame dans ces réunions festives?

\section{Un tissage de réseaux sociaux de plus en plus onéreux}

Ruffier qualifie les fêtes ouzbèkes, d'« espaces de construction des identités et des solidarités ». Cela représente bien l'enjeu principal d'un mariage. Au-delà de la construction d'un foyer pour les époux, qui fondent leur propre famille, le mariage en lui-même est la vitrine de la situation sociale des parents, de leur capacité à réunir des gens et à les recevoir avec faste. Le capital financier est prépondérant, puisque les sommes engagées sont considérables. Le repas est pantagruélique : des animaux sont généralement abattus pour confectionner les plats principaux, dont l'incontournable plov. En outre, les boissons, alcoolisées et non-alcoolisées, sont servies à foison, un orchestre anime la plupart du temps les festivités, des danseuses professionnelles sont embauchées, et le tout est souvent filmé par un caméraman engagé pour l'occasion.

Les invités ne se comptent pas. Dans les villages, des crieurs annoncent la date des réjouissances et tous les habitants qui connaissent un tant soit peu les protagonistes se déplaceront pour l'événement. Souvent, les invités, si on peut les nommer ainsi, quittent la table une fois leur repas avalé afin de céder leur place aux nouveaux arrivants, dans un mouvement incessant d'arrivées de nouveaux convives. Des tentatives de régulation des mariages ont été effectuées par le gouvernement, qui entendait limiter le nombre de convives, la quantité d'alcool proposée ainsi que le montant général des dépenses. Mais les lois sont une fois de plus contournées. Les responsables du comité des mahalla, chargés de faire respecter ces restrictions, s'avèrent le plus souvent complaisants et les mariages continuent à représenter une fête grandiose.

Pétric (2006 : 175) évoque l'existence de cahiers de comptes, appelés sharbatnoma, dans lesquels sont inscrits les cadeaux donnés et reçus. Ils permettent aux familles de garder trace des échanges, et de savoir ce qu'il convient de " rendre » à untel lors 
d'une prochaine festivité. Je fais ici référence au cycle du don (donner, recevoir, rendre), mis à jour par Mauss (2001 : 143-279). Les invités offrent divers présents aux jeunes mariés : tapis, matériel électroménager, argent, etc. En retour, ces derniers ou leurs parents donneront dans une prochaine fête un cadeau de valeur semblable ou supérieure.

Or ces cadeaux, chez les migrants, sont de plus en plus importants et de plus en plus chers. C'est ce que me confie Muhabat, alors que nous assistons ensemble au mariage d'une jeune fille de sa parenté. J'avais rencontré sa belle-sœur à Séoul et son frère à Samarkand. Le mariage fut pour moi l'occasion de faire connaissance avec plusieurs membres de cette famille qui bénéficie des retombées financières de la migration :

"Tu ne connais peut-être pas mes trois autres frères qui vont régulièrement à Séoul pour travailler? Ensemble, ils font du business de voiture, qu'ils envoient en Russie ou en Chine. Ils ont le visa du business, le D8. Deux de mes belles-sœurs [épouses de ses frères] sont aussi allées là-bas pour travailler. Moi aussi,j'aimerais partir là-bas avec mon mari. On a eu cinq filles, et les deux dernières sont des jumelles. Je voudrais m'arrêter là, mais mon mari veut un fils. Il va falloir qu'on pense à leur mariage, cela coûte cher. Et puis, cela nous coûte aussi très cher d'assister aux mariages des autres : on ne peut quand même pas venir les mains vides!

Ici, les salaires ne suffisent pas.

Tiens, regarde, c'est eux les parents de la mariée [Muhabat me montre du doigt la photo d'un couple qui apparaît sur un écran géant dans la salle de restaurant où nous nous trouvons]. Ça fait dix ans qu'ils sont partis en Corée, et qu'ils n'ont pas remis les pieds en Ouzbékistan. Depuis ses huit ans, la mariée [qui a dix-huit ans] vit avec ses grands-parents. C'est eux qui ont organisé le mariage pour elle. C'est un beau mariage, c'est sûr, mais quand même, les parents devraient être là ! Ce n'est pas dans nos traditions de se marier sans ses parents!».

Ainsi, une des causes de la migration peut être le besoin non seulement d'organiser pour ses enfants une fête digne, mais aussi de rendre les honneurs lorsque l'on est invité à une cérémonie. Ici, Muhabat est prise dans les réseaux de ses frères, tous migrants. Les cadeaux sont très onéreux, et Muhabat et son mari peinent à suivre le rythme effréné des dons. Enrichis, les migrants organisent des fêtes-vitrines, destinées à rendre visible leur nouveau statut financier. Ici, le cas est particulièrement intéressant, car les parents ont délégué à leurs propres parents l'organisation des festivités, qu'ils financent, mais auxquelles ils ne peuvent pas assister. Étant illégaux en Corée du Sud, mais y exerçant une activité lucrative, ils souhaitent y rester plus longtemps sans pouvoir revenir en Ouzbékistan, de crainte de se voir interdire de séjourner à l'avenir sur le territoire coréen.

\section{Logement et amélioration du cadre de vie}

Pour beaucoup de migrants, l'achat d'un logement représente un investissement important de l'argent économisé en migration. Il s'agit d'abord de s'assurer de rester dans un lieu qui leur appartient, d'offrir un toit à leur famille, mais aussi de s'ancrer dans un territoire spécifique. Enfin, il permet aussi aux migrants de donner à voir leur nouveau statut. 


\section{L'ancrage dans un territoire (ville et mahalla)}

Ainsi, pour certains migrants nés dans un village, posséder un logement dans la ville est un gage de non-retour au village. De nombreux qishloqi venus travailler dans une ville, comme j'ai pu le voir à Samarkand, rêvent de s'acheter un " appartement russe ", c'est-à-dire un appartement dans une barre d'immeubles. Il s'agit ici d'une représentation du confort, du luxe à l'occidentale avec «toutes les commodités », comme aiment à le souligner les néo-citadins : gaz, électricité, eau courante et même eau chaude, sanitaire relié au tout-à-l'égout. Se pose toutefois la question de l'autorisation, la propiska à obtenir avant de pouvoir s'installer ${ }^{12}$.

$\mathrm{Au}$ contraire, certains migrants reviennent de l'étranger en souhaitant s'acheter une « maison sur cour », parfois appelée aussi « maison ouzbèke », mais en périphérie de la ville, pas à la campagne. Il s'agit pour eux de revenir aux maisons " traditionnelles ", comme plusieurs d'entre eux me l'ont expliqué, mais en y apportant les commodités des « appartements russes ». La maison sur cour permet d'y organiser des festivités, et d'accueillir la famille élargie, ce qui n'est pas à proprement parler impossible dans un « appartement russe », mais moins confortable car plus exigu.

Le summum du luxe est représenté par les " maisons américaines ». Il s'agit de constructions modernes, souvent bâties sur mesure, imaginées par des architectes qui puisent dans les représentations du luxe à l'ouzbek. Frises, dorures, imitation de marbre, moulages en stuc, bois sculptés sont autant d'ornements indispensables. L'essentiel est de montrer la richesse des matériaux employés et d'y ajouter une touche occidentale : monumentale table en bois, escalier au milieu de la pièce, tonnelle en fer forgé, autant d'éléments de décoration souvent inutilisables ou peu fonctionnels, mais estimés par leurs propriétaires. J'ai ainsi été invitée à visiter une demeure de Namangan, construite grâce à l'argent envoyé par un migrant à Séoul. C'est Alikul qui me guide, fier de me faire découvrir la demeure de sa grand-mère :

"Voilà ma grand-mère, mon oncle, sa femme et leurs enfants [nous sommes dans une bâtisse à l'entrée de la propriété. Elle comporte deux pièces. C'est le seul bâtiment éclairé à notre arrivée]. Vous allez voir maintenant la maison, comme elle est immense. C'est une maison américaine, comme dans les feuilletons. [La visite se poursuit, avec toute la famille. Ils ne savent pas toujours où se trouvent les interrupteurs permettant d'éclairer les différentes pièces que nous visitons. Ils attendent visiblement ma réaction devant le majestueux escalier en colimaçon qui trône au milieu du séjour et permet d'accéder à la mezzanine.] Voilà, c'est notre maison

coréenne!".

12 Article de Roberta Cohen, «Freedom of movement », United States Mission to the OSCE (US Delegation to the Human Dimension Implementation Meeting), 07/10/2003: «In Uzbekistan, the government [...] severely limits the right to change permanent residence in country. Permission from local authorities is required in order to resettle in a new city, and the authorities rarely grant permission to those who wish to move to Tashkent. Local observers report that a bribe of roughly $\$ 100$ (96,529 soum) is necessary to obtain the required registration documents. Again, this is contrary to Uzbekistan's Vienna commitment that everyone has the right to freedom of movement and residence within the borders of each State ", http://osce.usmission.gov/archive/2003/10/ FREEDOM_OF_MOVEMENT.pdf, consulté le 24/07/2008. 
La maison américaine est devenue, en fin de visite, une maison coréenne. En réalité, j'ai entendu plusieurs fois la première expression, plus rarement la seconde. Il s'agit de désigner un logement directement inspiré des lieux de tournage de feuilletons américains, comme me l'ont affirmé plusieurs migrants. En réalité, il s'agit moins d'un habitat que d'un décor. Ici, personne ne dormait dans la maison principale, la «maison américaine » : la grand-mère, son fils, sa bru et ses quatre petits-enfants vivent dans la petite bâtisse de l'entrée. Il s'agit de paraître, la maison s'avérant fonctionner comme une vitrine de la réussite du migrant.

L'importance prise par l'escalier m'a beaucoup surprise, tant son aspect imposant me paraissait incongru, car il occupait le milieu d'un salon, comme s'il en constituait l'attrait principal. Je pense qu'en fait, il sert particulièrement à y prendre des photos, et représente en lui-même ce que mes interlocuteurs estiment être le design américain. J'ai compris cela en me rendant avec Dilnoza chez un photographe de Samarkand. Elle souhaitait faire parvenir à son époux, qui travaille à Séoul, son portrait. Avant d'être photographiée par le professionnel, elle devait choisir le décor qui servirait de toile de fond à son portrait. Le choix était large : gratte-ciels américains, Tour Eiffel, cottage anglais, palmiers, jardins européens, paysages de montagne, vue d'Hollywood. Dilnoza choisit finalement deux fonds : l'un représente une luxueuse voiture de sport garée devant une maison, l'autre est un cliché où figure l'intérieur d'une maison, le tout faisant, comme le dit Dilnoza elle-même, " très américain ». Dilnoza a emmené des vêtements pour pouvoir se changer. Elle désirait être prise en photo dans un ensemble veste et pantalon blancs, et a donc laissé sa robe de tissu ouzbek de côté le temps que le photographe l'immortalise.

Une hiérarchisation officieuse des logements serait la suivante : maison ouzbèke dans la campagne, appartement russe dans la ville, maison américaine (ou coréenne) dans la ville, maison ouzbèke dans la ville. Cette échelle renvoie à la hiérarchisation des pays d'accueil : la Russie, puis la Corée, puis les États-Unis, destinations du moins au plus facile d'accès et du moins au plus lucratives. Plus loin les migrants partent, plus importante sera la somme rapportée. Et parallèlement, plus ils émigrent loin, plus les logements achetés en Ouzbékistan seront prestigieux et éloignés de la campagne. La maison américaine semble donc être une demeure vitrine, symbole du changement de statut.

\section{Des logements prestigieux qui restent vides}

Au cours de mes terrains dans les trois destinations, il m'est très souvent arrivée d'être invitée par mes interlocuteurs à rendre visite à leur famille en Ouzbékistan. Mais j'ai été très surprise par le nombre de migrants qui me proposaient de disposer d'un logement resté vacant en leur absence. Ce fut le cas avec Robiâ :

Robiâ : "Si vous voulez, vous pouvez téléphoner à mes parents quand vous serez à Samarkand. J'expliquerai à ma mère que l'on s'est rencontré ici et elle sera ravie d'accueillir un mehmon [o. : invité] de France! Elle vit avec mes enfants, vous les verrez comme ça! Ils me manquent tellement. "

S. : "Ils habitent où, dans Samarkand?»

Robiâ : "On leur a acheté une maison au Registan [sous-entendu : avec l'argent gagné en migration par elle et son mari]. Mais on a aussi un appartement rue Gagarine, près de la gare. Vous pourrez loger là si vous voulez, il n'y a personne qui l'occupe pour l'instant ».

REMI 2010 (26) 3 pp. 59-84 
Deux logements ont donc été achetés grâce à l'argent de la migration. Un seul est utilisé par la famille. L'autre, l'appartement, reste vide, en attendant que les migrants regagnent le pays d'origine. Le discours est identique de la part de Samira :

"Mes parents sont à Samarkand, mais je vois ma mère environ une fois par mois, car elle vient ici pour faire du business. Moi-même je reviens à peu près deux ou trois fois par an en Ouzbékistan. On a trois maisons à Samarkand, mais elles sont vides, car mes parents préfèrent vivre dans leur maison. Donc si vous allez à

Samarkand, dites-le-nous ».

Comme le rappelle G. Pincent (2008), « depuis l'indépendance de l'Ouzbékistan en 1991, la notion de "propriété privée" a pris un sens nouveau. Certes, le sol appartient à l'État. Mais les murs des habitations sont détenus par la population. Leurs propriétaires considèrent qu'ils peuvent en faire ce qu'ils veulent. On assiste donc depuis 1991 à une explosion des chantiers dans les villes centrasiatiques [...]. Il existe [...] un système de contrôle des constructions, reposant sur le permis de construire. Mais beaucoup contournent ces lois. L'accès à la propriété privée, même limité par un labyrinthe administratif, a ainsi encouragé les transformations totales ou partielles opérées dans les maisons traditionnelles. Il faut les réparer, les agrandir, améliorer leur confort ou montrer la richesse familiale ».

Cette course à l'amélioration de la demeure semble avoir davantage pour but de posséder un bien visible par tous et prestigieux que de l'habiter réellement. Il peut s'agir d'un investissement pour le futur, comme Tursun :

«Je me suis fait construire une maison à Kokand, pour plus tard, quand je serai marié. Pour l'instant, elle est vide. Quand je rentrerai, je préférerais vivre chez mes parents d'abord, et après, on verra ».

Ces maisons sans habitants ne doivent pas pourtant apparaître comme inutiles, puisqu'elles servent à s'attirer une certaine reconnaissance sociale.

\section{Vers un nouveau mode d'habitat?}

Vivre dans un nouveau logement, que l'on vient d'acquérir, équivaut aussi bien souvent à changer ses habitudes domestiques. Tout d'abord, il peut s'agir de changer de lieu de vie, d'habiter désormais dans une ville différente de celle où le migrant séjournait avant le départ. Mais surtout, la forme du logement et son aménagement peuvent différer. Ainsi, habiter un appartement russe en centre-ville de Tachkent signifie-t-il changer significativement de mode d'habitat pour celui qui est né dans une maison en terre d'un qishloq.

L'aménagement est censé montrer, une fois de plus la richesse des propriétaires. Bekzod s'est ainsi acheté un appartement pour lui, à Tachkent, et a fait construire chez ses parents, à Qarchi, un sauna à l'extérieur de la maison. Quand je me suis rendue chez eux, j'ai pu constater qu'en effet, une magnifique pièce en bois tout à fait fonctionnelle, semble-t-il, se dressait dans le jardin. Les parents de Bekzod me l'ont fait visiter, mais il m’a semblé que le sauna n'était pas très souvent utilisé. De la même façon, voici ce que m'a confié Nodira, qui habite au cinquième étage d'un grand immeuble soviétique dans 
Tachkent et me fait visiter son appartement :

"Vous voyez, il y a dix pièces. Depuis deux ans, j'ai fait beaucoup de travaux dans cet appartement, sans le dire à Ališer. Il aura la surprise quand il rentrera [de New York]. Je ne m'attendais pas à ce que ce soit aussi dur de diriger des travaux, quand on est une femme! Mais je ne me plains pas, j'ai bientôt terminé ce que je voulais faire. Seulement, je voulais installer un jacuzzi et il y a deux problèmes. Déjà, ça ne passait pas dans l'ascenseur, et dix hommes auraient été nécessaires pour le monter par l'escalier. Et puis de toute façon, ça risquait de faire sauter l'électricité à chaque fois qu'on l'allume. C'est une machine qui consomme beaucoup d'énergie. Donc j'ai préféré finalement faire installer une baignoire à trois angles. C'est aussi bien comme ça. Mais qu'est-ce que c'est fatigant de s'occuper de tout ça, et de voir l'appartement sens dessus dessous pendant les travaux! ».

Cet exemple est assez étonnant, en ce qu'il nous montre une femme qui décide seule de l'utilisation de l'argent envoyé par son époux qui travaille à New York. Elle prend en charge les travaux et la transformation de l'appartement en un luxueux logement, sans en avertir son époux.

Enfin, des installations informatiques sont prévues dans plusieurs logements de migrants. Ainsi, Ziëd s'est-il acheté à Séoul un ordinateur et a-t-il fait installer une connexion Internet à domicile pour que lui, ses enfants et le reste de la famille puissent en profiter. De même, Tahir a fait acheter à ses parents, depuis New York, un ordinateur fixe et un ordinateur portable. L'aménagement des logements consiste donc à faire voir des objets de valeurs, pour démontrer aux visiteurs l'importance de la richesse de ses hôtes. Les voitures jouent aussi ce rôle.

Parfois, ce sont aussi des signes qui rappellent le pays d'accueil qui se trouvent exposés par les familles. Les photos touristiques, par exemple, sont parfois mises sous cadre et montrées. Dans la famille de Bekzod, la présence d'un parent aux États-Unis est particulièrement valorisée, et un autocollant aux couleurs de Columbia University a été placé à l'arrière de la voiture. Dans la famille de Tursun, l'appareil servant à cuire le riz, ramené de Corée, est souvent montré aux invités, comme une curiosité. Dès lors, il semble que le logement puisse être envisagé comme une vitrine de réussite matérielle par le passage à l'étranger.

\section{CONCLUSION}

Devenu « nouvel Ouzbek », l'ex-migrant n'informe personne de ce qui s'est réellement déroulé à l'étranger. Il rentre enrichi, et la manière dont il a perçu cet argent n'est jamais éclairée. La zone d'ombre de l'entre-deux, de la période liminaire qu'est la migration, est laissée de côté, au profit de l'insistance sur les marques du nouveau statut social des ex-migrants, reconnus comme sortis victorieux de leur initiation secrète.

Les migrants de retour dans leur pays d'origine semblent avoir acquis une grande visibilité et du fait de leur richesse sont investis d'une mission particulière pour s'associer 
à toutes sortes de projets. Ce dynamisme pourrait en quelque sorte être considéré comme une contrepartie à l'abandon de la patrie : partis loin de chez eux pendant de longues années, ils en reviennent plus attachés encore à leur ouzbékité, et déterminés à jouer un rôle prépondérant dans l'Ouzbékistan post-soviétique. Ainsi, les rapports de pouvoir sont redéfinis au sein de la famille. Les migrants ne peuvent retrouver la même position de père, d'époux et de fils qu'ils occupaient avant leur départ, alors que la maison a fonctionné sans eux pendant parfois de nombreuses années. Ils changent de statut au niveau du mahalla car, devenus « nouveaux Ouzbeks », c'est-à-dire enrichis, ils tissent un réseau d'interdépendance qui les place en position de force. Sollicités pour leur argent, leur capital social s'accroît considérablement.

D’un autre côté, ceux qui n'ont pas amassé un capital suffisant pour partir, s'enfoncent dans le néant, et perdent leur identité sociale. Quant aux familles des migrants, elles suivent une trajectoire identitaire tout à fait surprenante, en acquérant toutes les apparences de l'occidentalisation, rigoureusement à l'inverse des migrants de retour.

À partir de ce paradoxe se dégage la conclusion suivante : en accomplissant le rituel migratoire, les migrants se constituent une identité de transition : ils bouleversent les rapports sociaux et la hiérarchie domestique, et pourtant deviennent les garants d'une ouzbékité imaginée; ils vont à l'étranger, mais sont indifférents aux valeurs des pays où ils ont vécu et permettent, grâce aux capitaux rapportés, de procéder aux rites qui marquent le plus fortement l'ouzbékité (mariage, réseau de dons et contre-dons, etc.). Au contraire, ceux qui sont restés ne se situent pas dans cette identité de transition, mais continuent d'imaginer un Occident dont il faudrait adopter les valeurs, au détriment de sa propre identité.

\section{Références bibliographiques}

ADELKHAH Fariba et OLSZEWSKA Zuzanna (2006) Les Afghans iraniens, Études du CERI, 125, $42 \mathrm{p}$.

ALTHANS Birgit, AUDHEIM Kathrin, WULF Christoph, et al. (2004) Penser les pratiques sociales comme rituels : ethnographie et genèse de communautés, Paris, L'Harmattan.

AUGÉ Marc et Daniel FABRE (1987) D'un rite à l'autre. Entretien entre Marc Augé et Daniel Fabre, Terrain, 8, dossier « Rituels contemporains », pp. 71-76.

BELL Catherine (1992) Ritual theory, ritual practice, New York, Oxford, Oxford University Press.

BELMONT Nicole (1986) La notion de rite de passage, in Pierre Centlivres et Jacques Hainard Dir., Les rites de passage aujourd'hui. Actes du colloque de Neuchâtel 1981, Lausanne, L'Age d'homme, pp. 9-17.

BENNEGADI Rachid et BOURDILLON François (1990) La santé des travailleurs migrants en France : aspects médico-sociaux et anthropologiques, Revue Européenne des migrations internationales, 6 (3), pp. 129-144.

BOURDIEU Pierre (1982) Les rites comme actes d'institution, Actes de la Recherche en Sciences Sociales, 43 (1), pp. 58-63.

CENTLIVRES Pierre (2000) Portée et limites de la notion de diaspora, Cahiers d'Études sur la Méditerranée et le Monde Turco-Iranien, 30, pp. 5-12. 
FATHI Habiba (2004) Femmes d'autorité dans l'Asie centrale contemporaine. Quête des ancêtres et recompositions identitaires dans l'islam postsoviétique, Maisonneuve \& Larose, IFEAC, Paris, $348 \mathrm{p}$.

GLUCKMAN Max (1962) Les rites de passage, in Max Gluckman Dir., Essays on the ritual of social relations, Manchester, Manchester University Press.

KAUFMANN Jean-Claude (2006) L'entretien compréhensif, Paris, Armand Colin.

LARUELLE Marlène (2006) Le nouveau rôle de la Russie en Asie centrale : les migrations de travail des Centre-asiatiques vers la Fédération russe, La Revue internationale et stratégique, 64 (4), pp. 133-141.

MASSOT Sophie (2009) Enjeux politiques et identitaires dans la migration internationale des Ouzbeks vers trois pôles urbains (Moscou, Séoul, New York), thèse de doctorat ès Sciences Politiques, IEP/FNSP-CERI, Paris, sous la direction de Dominique Colas.

MAUSS Marcel (2007) Essai sur le don. Forme et raison de l'échange dans les sociétés archaïques, Réédition du texte paru en 1923-1924 dans l'Année sociologique, Quadrige, Presses Universitaires de France, Paris, introduction par Florence Weber.

PÉTRIC Boris-Mathieu (2002) Pouvoir, don et réseaux en Ouzbékistan post-soviétique, Paris, Presses Universitaires de France-Le Monde (Partage du savoir), 298 p.

PINCENT Guillemette, La réhabilitation fonctionnelle des villes pré-coloniales d'Ouzbékistan : un outil de domination politique ?, http://www.cybergeo.eu/index1750.html, consulté le $24 / 07 / 2008$.

POUJOL Catherine (2005) Ouzbékistan : la croisée des chemins, Paris, Belin, La Documentation française (Asie plurielle).

POUJOL Catherine (2006) Santé mentale et société en Asie centrale post-soviétique ou comment tenter de diagnostiquer un mal-être social ?, in Outre-Terre (dossier Asie antérieure), 16 (3), pp. 367-380.

REGAMEY Amandine (1999) A. Loukachenka au miroir des histoires drôles. http://www.cerisciencespo.com/archive/septem99/artar.pdf.

RUFFIER Arnaud (2007) Samarcand. Identités et espaces festifs en Ouzbékistan, Paris, Aux lieux d'être (Documents).

RUFFIER Arnaud (2002) To'y, gap, ziyofat et bayram, espaces de construction des identités et des solidarités en Ouzbékistan, thèse à l'école doctorale Asie mineure et intérieure, EHESS, sous la direction de Gilles Veinstein.

SEGALEN Martine (2005) Rites et rituels contemporains, Paris, Armand Colin (128).

TEMINE Émile (1999) France, terre d'immigration, Paris, Gallimard (Découvertes Gallimard/ Histoire).

VAN GENNEP Arnold (1981) Les rites de passage. Étude systématique des rites, Paris, A.-J. Picard.

WINKIN Yves (2005) La notion de rituel chez Goffman. De la cérémonie à la séquence, Hermès, 43, dossier Rituels, pp. 69-78.

WULF Christoph (2005) Introduction. Rituels. Performativité et dynamique des pratiques sociales, Hermès, 43, dossier Rituels, pp. 9-22.

WRIGHT Richard (1994) Rite of passage, New York, HarperCollins Publishers. 


\title{
Le retour des migrants ou l'émergence des «nouveaux Ouzbeks » : les effets d'un rite de transition
}

\author{
Sophie MASSOT
}

Les migrations économiques contemporaines des Ouzbeks vers Séoul, Moscou et New York mettent en jeu des réseaux socio-familiaux, qui placent le migrant, avant, pendant et après sa migration au cœur d'un rite de transition qui permet de faire émerger, lors du retour, la catégorie socio-économique des « nouveaux Ouzbeks ». L'agrégation du migrant à un groupe est l'enjeu crucial lors du retour, via l'initiation qu'a constituée la migration et dont il est sorti victorieux. L'article explore les changements de fonction et d'attitude des différents membres de la parenté qui conduisent le migrant à donner un sens à son nouveau statut social. L'auteure s'intéresse aussi aux placements de l'argent amassé pendant la migration. Deux postes de dépenses importants apparaissent : l'organisation de célébrations fastueuses et l'acquisition de logements prestigieux, qui servent à être vu et reconnu en tant que personne riche, en tant que « nouvel Ouzbek ». Enfin, l'argent des migrations peut être utilisé pour favoriser de nouvelles mobilités, celles notamment de personnes de l'entourage des ex-migrants. Le rite de transition génère alors de nouveaux rites, malgré le silence du migrant sur ce qui s'est réellement déroulé à l'étranger.

\section{Return of the Migrants or Apparition of the "New Uzbeks": The Effects of a Transition Ritual}

\author{
Sophie MASSOT
}

The contemporary economic migrations of the Uzbeks to Seoul, Moscow and New York bring into play social and family networks, which place migrants, before, during and after their migrations, in the heart of a rite of transition enabling the emergency, on their return, of a socioeconomic category referred to as "the new Uzbeks". Joining a group is the crucial stake for the migrants on their return via the initiation that such a migration constituted and which he came out of victoriously. First of all, the changes in the duties and behaviors of the various members in their families lead the migrants to give sense to his new social status. Then, they ought to invest the money accumulated during the migration. Two important items of spending emerge then: the organization of sumptuous celebrations and acquiring prestigious accommodation whose aim is to "show off" and be considered as a wealthy person, as a "new Uzbek". Lastly, the money issued from these migrations may set off a renewal of mobility since it is often used for the departure of members of the ex-migrants' family circle: the rite of transition generates then new rites, even though the migrants keep silent on what really happened when they were abroad. 


\section{El retorno de migrantes o la emergencia de los "nuevos Uzbecos": los efectos de un rito de transición}

\section{Sophie MASSOT}

Las migraciones económicas contemporáneas de los Uzbekos hacia Seúl, Moscú y Nueva York construyen redes sociales y familiares que ponen el migrante, antes, durante y después su migración al centro de un rito de transición que permite hacer hincapié, cuando el migrante vuelve, en la categoría socio-económica de los «nuevos Uzbekos». El punto fundamental de la vuelta es la admisión del migrante a un grupo, tras la iniciación que constituye la migración que logró hacer. Primero, los cambios de funciones y actitudes de los varios miembros de la familia permiten al migrante encontrar el sentido de su nuevo estatuto social. Secundo, el migrante suele invertir el dinero ahorrado durante la migración. Dos categorías de gastos importantes aparecen: la organización de fiestas tremendas y la compra de alojamientos prestigiosos. La meta es que la gente ven y reconocen el migrante como une persona rica, es decir un «nuevo Uzbeko». Al fin y al cabo, el dinero de las migraciones permite una nueva movilidad, puesto que está utilizado para la migración de las personas que rodean a los ex-migrantes: el rito de transición es la base de nuevos ritos, aunque el migrante suela quedar mudo en cuanto a lo que realmente ocurrió al extranjero. 


\section{Kazakhstan, Kirghizstan : les politiques migratoires au regard des droits de l'homme}

\section{Amandine REGAMEY ${ }^{1}$ et Katherine BOOTH ${ }^{2}$}

n juin 2009 , la Fédération internationale des ligues des droits de l'homme a
mené une mission d'enquête sur les processus migratoires au Kazakhstan et au Kirghizstan ${ }^{3}$. Cet intérêt de la FIDH s'inscrit dans une attention croissante des organisations des droits de l'homme pour les droits des migrants alors que depuis des dizaines d'années, sur la scène internationale, s'élabore un ensemble de règles visant à définir et protéger de façon spécifique les droits des travailleurs migrants et de leur famille. À cet égard, la FIDH s'appuie en particulier sur la Convention internationale sur la protection des droits de tous les travailleurs migrants et des membres de leur famille, adoptée par l'Assemblée générale des Nations unies en 1990 ainsi que sur les Conventions 97 (1949)

1 Chercheuse rattachée au CERCEC, Chargée de mission au Bureau International de la FIDH, 17, passage de la Main d'Or, 75011 Paris ; regamey@univ-paris1.fr

2 Responsable du bureau « droit des migrants » à la FIDH, 17, passage de la Main d'Or, 75011 Paris.

3 La délégation était composée de Katherine Booth, responsable du Bureau « droits des migrants » et d'Amandine Regamey, chargée de mission auprès du Bureau international (FIDH) ainsi que de Sarah McKune, responsable juridique de l'organisation « Human Rights in China ». Dans les deux pays, la mission a rencontré des représentants des autorités, des organisations internationales, des organisations non gouvernementales ainsi que des travailleurs migrants et des réfugiés. Elle a bénéficié du soutien du « Kazakhstan International Bureau for Human Rights and Rule of Law » et du « Kyrgyz Committee for Human Rights ».

4 La Convention internationale sur la protection des droits de tous les travailleurs migrants et des membres de leur famille (Convention sur les travailleurs migrants ou CTM), adoptée en 1990, est considérée comme une des neuf conventions les plus importantes des Nations Unies en termes de protection des droits de l'homme. Elle vise à préciser l'application des droits prévus par les deux pactes internationaux relatifs aux droits civils et politiques et aux droits économiques, sociaux et culturels, à un groupe spécifique. La Convention pose des droits valables pour tous les migrants, y compris les migrants en situation irrégulière et énumère des obligations qui pèsent tant sur l'État d'emploi que sur l'État de transit et l'État d'origine. Elle précise certains droits supplémentaires pour les migrants en situation régulière qui ont, par exemple, droit au logement ou à la protection sociale, au même titre que les citoyens du pays, doivent être soumis à un même régime d'imposition et peuvent créer des syndicats. 
et 143 (1975) de l'Organisation internationale du travail ${ }^{5}$.

Le Kazakhstan est devenu, depuis 2004, un pays d'immigration (le neuvième au monde selon la Banque Mondiale), alors que le Kirghizstan reste essentiellement un pays d'émigration vers le Kazakhstan, mais surtout vers la Russie. Dans les deux pays, les migrations reflètent les évolutions économiques : à la croissance rapide du Kazakhstan, reposant en particulier sur l'exploitation des hydrocarbures, s'opposent les difficultés d'un Kirghizstan enclavé et pauvre en industries comme en ressources naturelles. Surtout, l'importance croissante des migrations constitue un défi pour les gouvernements qui ont dû mettre en place de nouvelles politiques migratoires et incite à se pencher sur la situation de milliers de personnes partant chaque année à la recherche d'un emploi et/ou de conditions de vie plus favorables.

Publié en octobre 2009, le rapport intitulé Kazakhstan/Kyrgyzstan: Exploitation of Migrant Workers, Protection Denied to Asylum Seekers soulignait à quel point le cadre législatif extrêmement restrictif mis en place par le Kazakhstan fragilise juridiquement les travailleurs migrants, ce qui les expose à des formes d'exploitation, y compris au travail forcé. Si la politique migratoire du Kirghizstan s'inspire des mêmes principes, c'est surtout la responsabilité des autorités de protéger leurs ressortissants qui est soulignée dans le rapport $^{6}$. Des recommandations ont été présentées aux autorités des deux pays au mois de novembre 2009, à l'occasion d'un séminaire régional à Almaty regroupant plusieurs organisations de défense des droits de l'homme (FIDH, 2010).

Un an après la mission au Kazakhstan et au Kirghizstan, l'objectif de cet article est de faire le point sur l'évolution des politiques migratoires des deux pays et sur leur potentiel impact sur la situation et les droits des migrants ${ }^{7}$. Il s'agit aussi, plus généralement, de se demander dans quelle mesure les droits des migrants sont pris en compte par les États dans la mise en place des réformes et de la politique migratoire. On tentera de montrer à cet égard comment les deux pays sont marqués, tant au niveau des pratiques que des règles juridiques, par leur appartenance à l'espace postsoviétique, mais aussi comment ils réalisent une nouvelle insertion sur la scène internationale, dans laquelle les droits de

5 La Convention 97 pose le principe que le traitement appliqué aux travailleurs migrants en situation régulière ne doit pas être moins favorable que celui appliqué aux citoyens du pays, en ce qui concerne notamment : les procédures de recrutement, les conditions de vie et de travail, et l'accès à la justice et à la sécurité sociale. Complémentaire, la Convention 143 développe des lignes directrices concernant la gestion des migrations irrégulières et établit l'obligation des États de « respecter les droits fondamentaux de l'homme de tous les travailleurs migrants » (art. 1).

6 Une partie du rapport est consacrée à la question des réfugiés et des demandeurs d'asile, qui ne sera pas abordée dans cet article, les enjeux et les normes juridiques applicables étant très différents de la question des travailleurs migrants.

7 L'actualisation a été permise par des contacts avec les ONG rencontrées au Kazakhstan et au Kirghizstan, mais aussi par une nouvelle série de rencontres officielles au Kazakhstan et au Kirghizstan en novembre 2009. Par ailleurs, des représentants de la délégation kazakhe auprès du Comité des droits économiques, sociaux et culturels de l'ONU ont rencontré une délégation de la FIDH en mai 2010 à Genève et ont fourni des documents internes récents émanant du ministère du Travail. Enfin, l'actualisation a reposé sur une consultation régulière de la presse disponible sur Internet et sur la liste de diffusion du Social Research Center (SRC) de l'AUCA (American University of Central Asia). 
l'homme constituent un enjeu fondamental.

\section{KAZAKHSTAN}

Le Kazakhstan est devenu, depuis 2004, un pays d'immigration : négatif en 2002, le solde de la balance migratoire était déjà positif en 2005 [Conception pol. mig Kaz. 2007-2015], l'immigration venant essentiellement des pays voisins d'Asie centrale et de Chine. La politique migratoire kazakhe, qui se déploie depuis 2008 dans une situation de crise économique, permet d'étudier l'impact de la politique migratoire d'un pays d'accueil sur les droits des migrants. L'exemple du Kazakhstan est d'autant plus intéressant qu'il cherche à se faire reconnaître comme un acteur à part entière du concert des nations « civilisées » (il est le premier pays de l'ex-URSS à présider l'OSCE en 2010) et qu'il développe une véritable diplomatie des droits de l'homme (Fauve, 2010) ${ }^{8}$.

Il s'agira donc ici de voir comment la politique migratoire mise en place au Kazakhstan peut être qualifiée au regard des normes internationales des droits humains, en s'interrogeant tout d'abord sur le cadre international, avant de voir la politique migratoire elle-même et ses effets sur les droits économiques et sociaux de migrants qui restent dans leur immense majorité en situation irrégulière.

\section{Le cadre international : entre droits de l'homme et préoccupations sécuritaires}

Si le Kazakhstan a ratifié la majorité des conventions de protection des droits humains de l'ONU ${ }^{9}$, il n'a en revanche toujours pas ratifié la Convention internationale sur la protection des droits de tous les travailleurs migrants et des membres de leur famille. La ratification de cette Convention se heurte en effet, au niveau international, à de fortes

8 Le comportement du Kazakhstan à l'égard du Comité des droits économiques sociaux et culturels de l'ONU est d'ailleurs significatif de ses aspirations : rapport rendu en avance (le 13 novembre 2007, alors qu'il était attendu le 30 juin 2008), réponses détaillées aux questions additionnelles, délégation de dix-huit personnes dirigée par la vice-ministre du Travail. Si le Kazakhstan a donc tout fait pour montrer son respect envers les organes des droits de l'homme de l'ONU, cela n'a pas empêché qu'il soit critiqué pour son incapacité à saisir « l'esprit » des droits humains : "Vous parlez de développements, de buts économiques, de legal awareness, mais je ne vois pas de conscience des droits de l'homme dans tout ça. Les droits de l'homme, ce n'est pas tant la loi que les Hommes ; le problème n'est pas celui du développement, mais en quoi ce développement bénéficie aux gens » (Intervention de l'experte Virginia Bonoan-Dandan lors de la session du Comité DESC du 10 mai 2010, Genève. Notes personnelles).

9 Parmi lesquelles la Convention sur les droits de l'enfant (le 12 aout 1994) ; la Convention sur l'élimination de toutes les formes de discrimination à l'égard des femmes; la Convention contre la torture et la Convention sur l'élimination de toutes les formes de discrimination raciale (toutes les trois le 26 aout 1998). Plus récemment, le pays a ratifié le Pacte international relatif aux droits civils et politiques et le Pacte international relatif aux droits économiques, sociaux et culturels (le 24 janvier 2006). 
réticences émanant essentiellement des pays d'immigration ${ }^{10}$. Il est significatif à cet égard qu'en Asie centrale ce soit le Tadjikistan et le Kirghizistan, tous les deux pays exportateurs de main-d'œuvre, qui l'aient ratifiée. Le Kazakhstan n'a pas non plus ratifié les Conventions 97 et 143 sur les travailleurs migrants de l'Organisation Internationale du travail.

Le principal argument qu'avance le Kazakhstan pour repousser la ratification de la Convention sur les travailleurs migrants est d'ordre financier : " il y a trop de migrants au Kazakhstan pour qu'on puisse leur assurer l'accès au logement, aux écoles, aux hôpitaux » [Kaliuzhin 2009]. Asel Nusupova, vice-ministre du Travail a argué, elle, que les Conventions de l'ONU imposent à l'État l'obligation d'une réalisation pratique ${ }^{11}$ et qu'il convient donc, avant de ratifier, de « voir quels changements de la législation et quelles dépenses sont nécessaire. [...] Il vaut mieux attendre que tout soit prêt pour ratifier que de se rendre compte après que nous ne sommes pas capables de respecter cette convention » [Nusupova-Lepekha 2010]. Il est à noter cependant que la Convention sur les travailleurs migrants n'introduit pas de nouveaux droits, ceux-ci étant déjà garantis par des Conventions ratifiées par le Kazakhstan ${ }^{12}$. Ainsi, les Organes des traités chargés de vérifier le respect par les États de ces conventions peuvent se saisir de la question des droits des travailleurs migrants, comme l'a fait le Comité des droits économiques, sociaux et culturels en mai 2010 [CESCR Kaz 2010].

Pour repousser la ratification de la Convention sur les travailleurs migrants, les officiels kazakhs avancent aussi l'existence d'une Convention adoptée dans le cadre de la Communauté des États indépendants (CEI) qui serait «mieux adaptée au contexte régional» [Nusupova-Nurseitov 2009]. La « Convention sur le Statut légal des travailleurs migrants et des membres de leurs familles des États membres de la CEI », adoptée en 2008, a effectivement été ratifiée par le Kazakhstan le 30 décembre 2009. Néanmoins, cette convention ne vise pas tant la protection des droits que la « régulation » des flux migratoires afin qu'ils conviennent aux « intérêts socio-économiques des parties » (préambule). Mais surtout, à la différence de la Convention internationale, elle ne s'applique qu'aux

10 En juillet 2010, la Convention avait été ratifiée par quarante-trois États et signée par seize autres (la signature étant l'étape préliminaire à la ratification). Une campagne internationale lancée à l'occasion du vingtième anniversaire de son adoption par la FIDH et ses partenaires exhorte tous les États qui ne l'ont pas encore fait (dont l'ensemble des États de l'Union européenne) à ratifier cet instrument clé de protection des droits humains (voir http://www.fidh.org/Journee-Internationale-des-Migrants-2009). Pour un résumé des principaux arguments présentés par les États pour justifier leur refus de ratifier la Convention, voir le Guide sur la ratification de la Convention sur les travailleurs migrants, publié par le Comité international de pilotage de la campagne pour la ratification de cette convention, disponible sur www.migrantsrights.org/.

11 Tous les États ayant ratifié le Pacte doivent présenter régulièrement un rapport devant le Comité, composé d'experts indépendants, qui font un certain nombre de recommandations. À cet égard, les rapports des États devant les Comités ainsi que les rapports alternatifs des ONG constituent une source d'étude des politiques publiques souvent ignorée des chercheurs, qui sont toutes disponibles sur le site du Haut Commissariat aux droits de l'homme (www.ohchr.org).

12 Il est à noter que le Pacte international relatif aux droits civils et politiques et le Pacte international relatif aux droits économiques, sociaux et culturels vont même au-delà de la Convention sur les travailleurs migrants en termes de protection accordée aux migrants, puisqu'ils ne font aucune distinction entre les migrants en situation régulière ou irrégulière. 
personnes « résidant et travaillant légalement sur le territoire d'une des parties » (art. 1 et 2) et exclut donc les migrants en situation irrégulière. Ceux-ci sont au contraire visés par l'accord sur « la lutte contre les migrations illégales » adopté dix ans plus tôt déjà, en mars 1998.

En effet, si la vision des migrations qui domine au Kazakhstan est très marquée par des préoccupations sécuritaires et la volonté de lutter contre les migrations « illégales » (cf. infra), cette approche est encore renforcée par la participation du Kazakhstan à un certain nombre d'organisations régionales.

Ainsi l'Organisation de Coopération de Shanghai ${ }^{13}$, destinée à lutter contre le terrorisme, le séparatisme et l'extrémisme, prévoit également une coopération dans le domaine du contrôle des frontières et une lutte commune contre « d'autres formes d'activités criminelles transnationales et les migrations illégales » [Traité Shanghai 2007, art. 8]. De la même manière, dans le cadre de l'Organisation du Traité de Sécurité Collective ${ }^{14}$, la sécurité définie au sens large inclut les migrations illégales. Les États membres ont pour obligation de " coordonner et harmoniser leurs efforts pour combattre le terrorisme international et l'extrémisme », mais aussi « les migrations illégales et d'autres menaces à la sécurité des États membres » [Charte CSTO 2002, art. 8] et ils se réunissent régulièrement pour coordonner leurs efforts dans ce domaine.

\section{Une politique migratoire sélective}

La politique migratoire kazakhe, telle qu'elle est présentée par les autorités, répond à un double objectif : favoriser le retour des Oralman (les Kazakhs « ethniques » résidant dans d'autres États de la région) et faire venir des spécialistes nécessaires au développement de l'économie kazakhe tant que le pays n'a pas pu former ses propres spécialistes. Sans développer ici la question des Oralman, on peut relever que leur installation a lieu dans un cadre contrôlé à l'extrême (quotas, lieu d'installation fixé par le gouvernement). De même, le programme Nurly Kosh concernant tant les Oralman que les migrants internes vise à soumettre les processus migratoires à l'objectif de développement démographique et socio-économique des régions. Ainsi les migrations sont-elles envisagées non comme une possibilité laissée aux individus d'exercer leur droit à la liberté de mouvement, mais comme un moyen de réaliser les objectifs de développement du Kazakhstan pour les années à venir.

\section{Attirer les spécialistes étrangers et protéger le marché du travail}

La politique migratoire kazakhe est construite sur un objectif de protection du marché du travail interne. Cette politique de préférence nationale est posée par l'article 5

13 L'Organisation de Coopération de Shanghai réunit depuis 2001 la Chine, le Kazakhstan, la Russie, le Tadjikistan, l'Ouzbékistan et le Kirghizistan.

14 L'Organisation du Traité de Sécurité Collective réunit depuis 2002 l'Arménie, la Biélorussie, le Kazakhstan, le Kirghizistan, la Russie, le Tadjikistan et l'Ouzbékistan sur la base du Traité de Sécurité collective de la CEI de 1992. 
de la Loi de 1997 « Sur la migration de la population» qui stipule que « dans la régulation des migrations de travail, la protection du marché de travail interne a une signification prioritaire ». En juin 2009, la vice-ministre du Travail Asel Nusupova expliquait à la FIDH que «la priorité pour nous est de protéger nos travailleurs. Si un employeur veut inviter un travailleur migrant, il doit essayer de remplacer sa position par un spécialiste kazakhe dans les deux ou trois ans qui viennent » [Nusupova-Nourseitov 2009].

En effet, le Kazakhstan ne cherche à attirer des migrants et des travailleurs qualifiés que pour remplir les postes pour lesquels il n'y a pas de spécialistes kazakhes. Cette politique est clairement temporaire, puisque la formation des cadres (en particulier dans le cadre du programme Bolashak ${ }^{15}$ d'études à l'étranger) doit à terme permettre d'assurer le remplacement des étrangers par des Kazakhs. Ainsi le ministère du Travail tient-il précisément les comptes et note-t-il que dans les quatre premiers mois de 2010 : « 772 travailleurs étrangers ont été remplacés par des spécialistes locaux (contre 599 en 2009) » [Mintruda mai 2010].

L'objectif de protection du marché intérieur explique l'existence depuis 2001 de quotas pour la main-d'œuvre étrangère. Établis en pourcentage de la population active du Kazakhstan, ces quotas sont divisés en quatre catégories : directeurs (pervye rukovoditeli), spécialistes hautement qualifiés, travailleurs hautement qualifiés et, depuis 2006, travailleurs agricoles saisonniers. En 2008, le quota était établi à 1,6\% de la population active de la République, équivalent à 126000 personnes environ (OIM, 2009). En 2009, en raison de la crise, le quota a été réduit de moitié à l'instar de la Russie et s'est établi à $0,75 \%$ de la population active, soit 63483 personnes, et il a été maintenu à ce niveau en $2010^{16}$.

Cet objectif de préférence nationale explique également les règles qui s'appliquent aux employeurs voulant engager des étrangers (ILO, 2008 : 14-17). Ceux-ci doivent prouver qu'ils ont essayé de trouver un citoyen kazakh pour occuper ce poste (publication d'offres dans la presse, entretiens obligatoires avec les candidats locaux, recours aux bases de données locales des demandeurs d'emploi). Les autorisations délivrées à l'employeur peuvent être données sous conditions, celle d'offrir une formation à des employés kazakhs par exemple, et la liste des travailleurs migrants employés est encore soumise à autorisation. Ainsi, dans les deux premiers mois de 2010, 628 autorisations d'engager un étranger sur 4006 avaient été rejetées, dont dix-huit « car une offre existait déjà sur le marché de l'emploi » [Mintruda mai 2010]. La complexité du système est telle, que le plus souvent les

15 Le programme Bolashak sélectionne des étudiants kazakhs et finance leurs études à l'étranger dans les domaines des sciences, de l'industrie, de l'économie, de l'administration, de la médecine ou des sciences humaines. À leur retour, les étudiants doivent travailler cinq ans au Kazakhstan. Selon le site officiel du programme, entre 1994 et 2010, 6679 étudiants ont reçu une bourse internationale Bolashak (Avenir en kazakh, NDLR) et ont fait leurs études en particulier en GrandeBretagne, aux États-Unis, en Russie, en Allemagne, au Canada, en Malaisie ou en Chine (cf. http://edu-cip.kz/).

16 Ces $0,75 \%$ correspondent à l'addition des quatre catégories : $0,35 \%$ de la population active pour la première et la seconde catégorie, directeurs et spécialistes hautement qualifiés, $0,35 \%$ pour la troisième catégorie, travailleurs hautement qualifiés et $0,05 \%$ pour la quatrième catégorie, travailleurs agricoles saisonniers. 
quotas ne sont pas remplis, comme le reconnaissent d'ailleurs les autorités elles-mêmes ${ }^{17}$.

Une nouvelle loi sur les migrations est en discussion devant le parlement kazakhstanais ${ }^{18}$, dont les autorités assurent qu'elle " correspond mieux aux exigences internationales » et qu'elle «prend en compte les exigences et les inquiétudes des ONG» [Nusupova-Lepekha 2010]. De fait, d'après les informations parues dans la presse, il semble surtout qu'il s'agisse d'une loi visant à modifier le système de quotas. Une partie des autorisations de travail serait distribuée au niveau national, l'autre au niveau local, et " à côté du quota général, valable actuellement, seraient introduits des quotas par pays et par branche, ainsi que des quotas pour les projets prioritaires » [Abdykalova 2010]. Trois catégories de migrants seraient définies, les travailleurs étrangers, les travailleurs agricoles et les bizness-migranty. Seuls les deux premiers seraient invités selon le système des quotas, les autres pouvant mener une activité légale à condition de créer une personnalité juridique et de déposer en banque une certaine somme d'argent [Duisenova 2009].

\section{Des migrants maintenus en situation irrégulière}

Le système existant actuellement au Kazakhstan aboutit cependant à laisser totalement de côté l'essentiel des migrants, ceux qui viennent travailler en particulier dans la construction, dans l'agriculture et sur les marchés. Ainsi, alors que le nombre de travailleurs migrants résidant au Kazakhstan est évalué entre 500000 et 2 millions selon les experts, seuls 28673 travailleurs migrants travaillaient régulièrement pendant les quatre premiers mois de 2010 selon le ministère du Travail [Mintruda mai 2010].

Certes, les autorités reconnaissent elles-mêmes l'existence de migrations irrégulières. Dans une note officielle communiquée à la FIDH, le ministère du Travail notait son « inquiétude devant la présence de travailleurs migrants illégaux, travaillant essentiellement dans le sud du pays dans l'agriculture (tabac, coton) et la construction » et rapportait que « selon les données non officielles, rien que dans la construction à Astana travailleraient plus de 15000 citoyens ouzbeks » [Mintruda mai 2010]. Une campagne de régularisation avait d'ailleurs été organisée en 2006 et avait abouti à la régularisation de 164600 étrangers en trois mois, ce chiffre ne comprenant que ceux qui étaient entrés et résidaient légalement au Kazakhstan et qui avaient trouvé un employeur qui accepte de faire la demande pour eux. La nouvelle loi prévoit la possibilité de reconduire des régularisations de ce type tous les trois à cinq ans.

Elle prévoit également « d'élargir le quota pour les travailleurs migrants saisonniers, sans le limiter aux travaux agricoles et sans qu'il y ait besoin d'un accord international » comme c'est le cas actuellement [Mintruda mai 2010]. Il s'agirait sans doute d'un progrès permettant de contourner les difficultés à conclure un accord avec l'Ouzbékistan, en dépit du nombre important de travailleurs migrants ouzbeks travaillant notamment dans

17 «Pour 2009, les quotas de travail étaient de 80 000, on n'a finalement enregistré que 38000 personnes » [Nusupova-Lepekha 2010].

18 Selon les données disponibles sur le site du Parlement, elle a été déposée en janvier 2010 devant le Comité du développement socioculturel qui doit préparer ses conclusions d'ici au 29/10/2010 (http://www.parlam.kz/DocInfo.aspx?lan=ru-RU\&infdat=5\&page=2\&id=139). 
les champs de coton et de tabac au Kazakhstan.

Néanmoins, le problème principal reste le fait que selon la législation actuelle, les travailleurs migrants ne peuvent rien faire pour régulariser leur situation : seuls les employeurs ont la possibilité de demander pour eux des autorisations de travail. Aussi la recommandation principale de la FIDH aux autorités kazakhes était-elle de réviser le système d'autorisations de travail de manière à ce que les permis soient attribués aux migrants eux-mêmes. Le nouveau projet de loi sur les migrations prévoit apparemment qu'en plus d'une autorisation d'emploi de main-d'œuvre étrangère délivrée à l'employeur, une autorisation de travail soit délivrée au travailleur étranger, à condition que celui-ci ait un employeur. La capacité réelle qu'aura un employé de quitter son employeur reste cependant à déterminer.

S'il est indispensable de donner aux migrants la possibilité de légaliser eux-mêmes leur situation, la question des quotas reste en revanche difficile à évaluer d'un point de vue des normes des droits humains ${ }^{19}$. Le gouvernement kazakh fait explicitement référence à la politique migratoire des pays occidentaux, arguant du fait que le système de quotas, y compris par pays d'origine, existe aux États-Unis et «dans presque tous les pays d'Europe » [Duisenova 2009]. De fait, du point de vue du droit international, les États nationaux restent souverains dans la définition des règles d'admission s'appliquant sur leur territoire ${ }^{20}$. La Convention sur les travailleurs migrants reconnaît par ailleurs explicitement aux États le droit de réguler les migrations, et reconnaît même le droit à l'État de réserver certains emplois à ses nationaux (art. 52).

L'existence de quotas relève clairement d'une « perspective utilitariste », dans laquelle n'est acceptable que «l'étranger perçu comme rentable pour l'économie » comme le dénonce en France le collectif « Uni(e)s contre une immigration jetable (UCIJ) » dans une pétition de 2007. Mais cette approche ne provoque pas, dans l'ex-URSS, la même mobilisation politique et citoyenne que celle qu'a connue la France. Le système de quotas n'est d'ailleurs pas forcément contesté par les ONG des droits de l'homme de la région, comme l'a montré le séminaire organisé par la FIDH en novembre 2009 à Almaty. Pour certains des participants, les quotas limitant le nombre de travailleurs migrants devaient être abolis, car ils fragilisent les migrants et créent une discrimination. Pour eux, même d'un point de vue strictement utilitariste, les quotas sont contre-productifs, et contredisent la volonté proclamée de l'État de protéger les travailleurs nationaux : ils créent une immigration irrégulière et les migrants irréguliers, payés des salaires de misère, constituent en quelque sorte une " concurrence déloyale » sur le marché de travail. Pour d'autres participants en revanche, les quotas sont inhérents à toute politique migratoire, qui

19 Dans son rapport de 2009, la FIDH n'appelle pas à l'abolition des quotas, mais à l'augmentation de la flexibilité du système (délivrance de permis pour des périodes plus longues de trois à cinq ans, possibilité de travailler légalement sur la base d'une simple déclaration pour des périodes jusqu'à un mois, limitation de la liste des documents à fournir, etc.).

20 Il y a de ce point de vue un vide juridique - le droit de chaque homme de quitter son pays et d'y revenir est garanti par un certain nombre de textes : Déclaration universelle des droits de l'homme (art. 13) ; Pacte International relatif aux droits civils et politiques ICCPR (art. 12 (2) et (4)) ; Convention sur les Travailleurs Migrants (art. 8). En revanche, le droit de s'installer dans un autre pays n'est pas garanti. 
implique forcément des limitations et une sélection des migrants. L'abolition des quotas leur semblait une mesure trop radicale et ils appelaient plutôt à améliorer la situation des migrants dans le cadre existant.

\section{Les atteintes aux droits des travailleurs migrants irréguliers}

«Quand les migrants viennent nous voir pour qu'on les aide à se faire payer ce qui leur est dî, il y a tant d'incompréhension. La police nous dit : " mais c'est un migrant illégal, quels droits a-t-il ?" " [Abysheva 2009]. Cette difficulté à défendre les droits des travailleurs migrants est un constat partagé par toutes les ONG de la région. De fait, rencontrant de nombreuses difficultés pour régulariser leur situation et risquant toujours d'être expulsés, les travailleurs migrants en situation irrégulière sont les plus susceptibles d'être victimes de travail forcé et ont peu de possibilités de recours.

\section{Conditions d'entrée, de séjour et risques d'expulsion}

L'espace qui unit le Kazakhstan et les autres pays d'Asie centrale (mis à part le Turkménistan) est en principe un espace de libre circulation. Cela n'empêche pas le Kazakhstan, à l'instar des autres pays de la région, d'avoir maintenu depuis la fin de l'URSS un système administratif et policier très contraignant. La multiplication des règles est par ailleurs autant d'occasions de corruption.

Les migrants originaires d'Ouzbékistan, Tadjikistan ou Kirghizstan voulant entrer au Kazakhstan, n'ont pas besoin de visas et l'entrée est en principe libre : le voyageur se voit distribuer à l'entrée une " carte de migration » sur laquelle il écrit les données de son passeport et le but de son voyage. Néanmoins, selon tous les témoignages, le passage des frontières reste une épreuve, que ce soit en train ou en voiture : tracasseries administratives, contrôles abusifs, confiscation de marchandise et pots de vin sont de mise. Les migrants craignent également les extorsions de fonds à la frontière, quand ils reviennent avec leurs salaires et se méfient autant de la police ou des douanes des pays traversés que de celles de leur propre pays.

Le cas du passage de la frontière entre Tachkent (Ouzbékistan) et Chymkent (Kazakhstan), une frontière par ailleurs très poreuse, est à cet égard significatif. Selon le monitoring fait en 2006 par «Adilet », une ONG basée à Chymkent, qui coïncide avec des témoignages de journalistes [Kravets 2009], la corruption se manifeste à tous les niveaux : paiement des gardes-frontières pour passer sans contrôle, mais aussi paiement pour ne pas faire la queue, pour récupérer plus rapidement une « carte de migration » sur laquelle il est écrit en trois langues qu'elle est gratuite. La collusion entre des passeurs et les gardes-frontières est manifeste et certaines ONG craignent que les chauffeurs de taxi ne « vendent » les migrants qui rentrent chez eux aux gardes-frontières [Abysheva 2009].

Sana Syzim, ONG basée à Chymkent, note que la majorité des migrants qui s'adressent à eux ont traversé la frontière illégalement (Sana Syzim, 2009). Ils le font pour aller plus vite, éviter de payer des pots-de-vin et de subir les humiliations, mais aussi parce qu'ils ne veulent pas montrer leur passeport aux gardes-frontières ouzbeks, ou même ils 
préfèrent partir sans passeport plutôt que de se le voir confisqué à leur arrivée sur leur lieu de travail (cf. infra). En conséquence, ils n'ont pas la carte de migration et le tampon dans le passeport qui leur permettraient ensuite de s'enregistrer légalement.

L'enregistrement auprès de la police migratoire est en effet obligatoire et suppose que le migrant trouve un employeur, un ami, un parent ou un propriétaire qui le déclare. Depuis avril 2010, les migrants de la CEI doivent s'enregistrer dans un délai de cinq jours, alors qu'auparavant les citoyens kirghizes avaient la possibilité de résider sans enregistrement pendant quatre-vingt-dix jours (trente jours pour les Tadjikes). Le Comité des droits de l'homme de l'ONU considère que ce système hérité de la propiska soviétique viole le droit à la liberté de mouvement et le choix de la résidence tel que prévu par l'article $12 \mathrm{du}$ Pacte international relatif aux droits civils et politiques [HRC Kyrg 2000]. De plus, l'enregistrement «permanent » conditionne l'accès à un certain nombre de droits économiques et sociaux comme l'accès à la santé.

Enfin, les migrants résidant ou travaillant illégalement au Kazakhstan risquent une amende, une expulsion et une interdiction de séjour au Kazakhstan pendant cinq ans [art. 394 et 396 du Code des infractions administratives]. En 2009, selon les chiffres du MVD 18258 personnes ont été expulsées, dont 17164 citoyens de la CEI, parmi lesquels $73 \%$ vers l'Ouzbékistan (12 471 personnes) et $10 \%$ vers le Kirghizistan (1 692) [Mintruda mai 2010]. Dans la majorité des cas, les migrants irréguliers sont accusés d'une inadéquation entre le but indiqué sur leur carte de migration (le plus souvent « privé » ou « tourisme ») et leurs activités réelles.

Les conditions qui prévalent dans les centres de rétention, dans lesquels les migrants peuvent être retenus pendant des semaines, sont dénoncées par de nombreuses ONG. Ils sont en particulier détenus avec des personnes accusées de crimes ou condamnées, ce qui est contraire à l'article 12 (3) de la Convention sur les travailleurs migrants. Les possibilités d'appel d'une décision d'expulsion ne sont pas claires, et même des officiels reconnaissaient en juin 2009 que les conditions d'expulsion posaient des problèmes, car « nous ne faisons que les amener à la frontière et les mettre dehors, cela ne nous intéresse pas de savoir ce qui se passera après » [Nusupova-Nurseitov 2009]. Enfin, les règles administratives prévoient que l'expulsion a lieu en principe aux frais du migrant ou de la personne invitante, ce qui est contraire à l'article 9 de la Convention 143 de l'OIT.

\section{Risque de travail forcé}

En mai 2010, dans ses conclusions sur le Kazakhstan, le Comité des droits économiques, sociaux et culturels se dit « profondément préoccupé par la situation des travailleurs migrants employés sans contrat dans les plantations de tabac et qui sont, avec leurs familles, vulnérables à l'exploitation et aux mauvais traitements » [CESCR Kaz 2010]. Cette situation ne prévaut pas uniquement dans l'agriculture, mais peut se retrouver dans tous les secteurs, en particulier la construction et les services.

Les problèmes tiennent tout d'abord aux conditions de travail (travail du lever au coucher du soleil, sans jour de congé) et aux conditions de logement (le plus souvent sur le lieu de travail, dans des containers, des baraquements, des caves, des hangars, etc.). À cela 
s'ajoute l'absence d'assurance et de protection contre les accidents, un problème auquel se heurtent d'ailleurs également les migrants réguliers ${ }^{21}$.

Souvent, les migrants en situation irrégulière se voient confisquer leur passeport, par leur employeur ou par l'intermédiaire - brigadir - sous prétexte de les enregistrer ou simplement « de les garder en sécurité ». Des migrants dont les passeports ont été confisqués courent le risque d'être " revendus » à un autre employeur, sans avoir la possibilité de s'enfuir - comme le montrent les cas dont se sont occupés l'ONG Sana Syzim et le bureau du Kazakhstan International Bureau for Human Rights and Rule of Law à Chymkent (FIDH, 2009 : 50-51).

Les ONG sont souvent amenées à aider les migrants et à récupérer les salaires qui ne leur ont pas été versés - une tâche difficile alors que la plupart n'ont pas de contrat de travail, et parfois même peu d'informations sur leur employeur. La menace de dénoncer ces employeurs à la police est à double tranchant, car les migrants risquent d'être expulsés. En effet, la police se retourne plus souvent contre les migrants irréguliers que contre ceux qui les emploient illégalement. En 2008136000 étrangers ont été arrêtés et 17000 expulsés pour des infractions aux règles migratoires, alors que seuls 1800 employeurs ont été poursuivis pour l'emploi de migrants irréguliers (sans nécessairement être sanctionnés) [Tashimov 2009]. D'ailleurs, les employeurs menacent souvent eux-mêmes les migrants de les dénoncer à la police et peuvent même utiliser les raids de la police pour se débarrasser de ceux qu'ils ne souhaitent pas payer.

Si les travailleurs migrants peuvent être privés de liberté ou forcés de conduire des activités illégales, il n'est pas besoin qu'une violence physique s'exerce contre eux pour que les conditions de travail décrites ci-dessus soient qualifiées de « travail forcé ». En effet, comme le rappelle un rapport de l'Organisation Internationale du Travail en 2005, le travail forcé suppose que "le travail ou le service exigé est exécuté sous la menace d'une peine », qui peut être «d'ordre psychologique : travailleurs en situation illégale menacés d'être dénoncés à la police ou aux services d'immigration » ou d'ordre financier "prélèvement d'une partie du salaire pour le remboursement des dettes, nonpaiement du salaire, perte de salaire accompagnée d'une menace de licenciement [...] ; [les employeurs] exigent parfois des travailleurs qu'ils leur remettent leurs pièces d'identité, ou ils les contraignent à travailler en les menaçant de confisquer lesdits documents » (OIT, $2005: 6$ ).

Les ONG soulignent aussi la difficulté à défendre des migrants qui sont eux-mêmes dans la majorité des cas prêts à travailler illégalement et qui sont tellement habitués à ces conditions de travail qu'ils remettent d'eux-mêmes leur passeport à l'employeur. Néanmoins, comme le rappelle le rapport de l'OIT, «les victimes du travail forcé sont fréquemment des personnes qui, initialement, se sont engagées de leur plein gré dans un travail-même s'il a fallu pour cela abuser de leur confiance-et qui comprennent plus

21 Comme l'a raconté Alisher, migrant d'Ouzbékistan résidant et travaillant légalement à Astana : «Si je suis malade ou que j'ai un accident au travail, je ne peux compter que sur moi-même. Je ne peux pas aller dans un hôpital d'état, quant aux hôpitaux privés, ils sont trop chers. Tout dépend de l'entreprise [...] Si c'est une entreprise correcte, ils paieront pour le traitement... sinon, ils te donneront quelques pilules » (Entretien FIDH, Astana, 12 juin 2009). 
tard qu'elles ne sont plus libres de le quitter. [On peut] considérer que le consentement initial est sans valeur s'il a été obtenu par une escroquerie ou un abus de confiance » (OIT, $2005: 6)$.

\section{KIRGHIZSTAN}

Le Kirghizstan accueille un certain nombre de migrants de travail, venus essentiellement des pays de la CEI et de Chine, et connaît également des migrations pendulaires entre l'Ouzbékistan et le sud du Kirghizstan. Si l'ampleur de ces migrations est minime, les modalités de gestion en sont intéressantes ; elles montrent à quel point le Kirghizstan et le Kazakhstan appartiennent à un espace commun, que l'on peut qualifier de postsoviétique en raison des nombreux parallèles existants avec la Russie également.

Le Kirghizistan a établi des quotas de travail pour les étrangers et les a réduits avec la crise comme ses deux grands voisins : pour 2010, le quota de travailleurs migrants s'établissait à 1758 personnes [24.KG 28/01/2010]. Alors que la volonté est avant tout d'attirer des investisseurs étrangers, le Kirghizstan soutient une politique de préférence nationale pour l'emploi et l'article 2 de la Loi sur les migrations de travail extérieures de 2006 pose « le droit prioritaire des citoyens Kirghizes à occuper les places vacantes sur le marché national du travail ». Les flux migratoires, qu'ils soient internes ou venus de l'extérieur, sont gérés également avec la pratique de l'enregistrement, qui là aussi conditionne l'accès aux droits économiques et sociaux. Comme le Kazakhstan, le Kirghizstan a mis en place une politique visant à faire revenir les Kirghizes « ethniques » (Kayrylman) vivant dans les pays voisins. Enfin, il est également membre de la Communauté des États Indépendants (CEI), de l'Organisation de Shanghai et de l'Organisation du Traité de Sécurité Collective. L'approche sécuritaire des migrations y domine aussi, comme le montre le fait que le neuvième bureau du ministère de l'Intérieur Kirghize est à la fois responsable des questions de terrorisme, d'extrémisme et de migrations illégales.

Le Kirghizstan reste néanmoins avant tout un pays de départ. Nous nous pencherons donc dans cette partie sur la politique migratoire du Kirghizstan en tant qu'État d'émigration, pour voir quelle responsabilité pèse sur l'État au regard des normes internationales, quelles mesures il peut mettre en place pour protéger ses citoyens à l'étranger, et enfin quelles difficultés il peut rencontrer, en prenant l'exemple des relations entre le Kazakhstan et le Kirghizstan.

Rappelons aussi que depuis la mission de la FIDH en juin 2009 et les entretiens officiels menés en novembre 2009, le Kirghizstan a connu une évolution politique mouvementée et violente. En avril 2010, des manifestations de protestation qui ont fait plus de quatre-vingt morts ont obligé le président Bakiev à s'enfuir. Le nouveau gouvernement, formé d'anciens membres de l'opposition et dirigé par Rosa Otumbaeva, a prévu un référendum pour l'adoption d'une nouvelle constitution qui limiterait les pouvoirs présidentiels et la domination d'un parti. Organisé le 27 juin, ce référendum (approuvé à $90 \%$ ) a eu lieu dans un climat de violence extrême dans le sud du pays, où les émeutes dirigées essentiellement contre les Ouzbeks ont fait des centaines de morts et près de 400000 réfugiés ou déplacés. 


\section{Préparer le départ et le retour des migrants}

Alors qu'on estime le nombre de travailleurs migrants quittant chaque année le Kirghizstan entre 400000 et 800000 personnes, les destinations privilégiées restent la Russie et le Kazakhstan. Le Kirghizstan dispose d'institutions et de textes destinés à mettre en place la politique migratoire, ce qui laisse penser que les gouvernements successifs ont pris la mesure de l'importance des migrations pour le pays.

Créé en 2005, le Comité d'État pour les Migrations et l'Emploi a été transformé en 2009 en ministère à part entière, passant de 500 à 4000 collaborateurs en quelques mois. Le nouveau gouvernement créé après la chute du président Bakiev conserve d'ailleurs ce ministère du Travail, de 1'Emploi et des Migrations.

Le Kirghizstan s'est par ailleurs doté depuis plusieurs années d'un arsenal législatif ainsi que de textes définissant la politique migratoire du pays. La « Conception de la politique migratoire de l'État pour 2005-2010» définit la politique migratoire comme une priorité, prévoyant que l'intégration du Kirghizstan dans le marché international du travail s'accompagnera d'une augmentation de l'exportation de la force de travail. La loi de la République Kirghize « sur les migrations de travail extérieures » du 13 janvier 2006 pose les conditions de départ des travailleurs migrants et leur protection ainsi que les conditions d'emploi des travailleurs étrangers au Kirghizstan. Enfin, le « Programme National de la République Kirghize sur la Régulation des processus migratoires pour 2007-2010 » définit les actions à entreprendre pour améliorer la gestion des migrations internes et externes : renforcer les institutions en charge, la coopération internationale, les représentations consulaires et l'information disponible pour les citoyens kirghizes qui partent, créer un système d'assurance, soutenir l'éducation et la formation professionnelle, et enfin améliorer la connaissance des flux migratoires.

Ce dernier point est en effet un problème, car l'État ne dispose pas de statistiques précises et fiables. En 2009, il était impossible d'avoir une évaluation précise du nombre de départs, car sur quarante-trois postes-frontières seuls quatre avaient la capacité d'enregistrer les migrants, et le Comité des Migrations se reposait toujours sur les statistiques fournies par le Service Fédéral des Migrations de Russie [Ryskulova 2009].

\section{Favoriser, accompagner ou lutter contre les migrations?}

«Nous ne stimulons pas les migrations, mais nous devons défendre les droits de nos citoyens qui sont des travailleurs migrants. En même temps, nous essayons de créer de bonnes opportunités de travail ici » a déclaré en juin 2009 Aygul Ryskulova, responsable du Comité des migrations et de l'emploi, à la mission de la FIDH. Comme tous les États d'émigration, le Kirghizstan doit en effet définir s'il cherche à favoriser les migrations, les freiner ou les orienter pour en tirer le meilleur parti.

Les conséquences négatives de ces migrations étaient soulignées en 2004 dans la «Conception de la politique migratoire pour 2005-2010» qui estimait que la balance migratoire négative avait absorbé « près de la moitié de la croissance naturelle de la République », ce qui a entraîné « une diminution de la force de travail qualifié et 
une baisse du potentiel scientifique, intellectuel et économique de la République ». Les migrations ont des conséquences sociales et économiques négatives en raison du départ d'une grande partie de la population d'âge actif (villages où ne restent que des femmes, des personnes âgées et des enfants), et les fonds transférés par les migrants ne favorisent pas systématiquement le développement économique du pays. Dans le même temps, les migrations sont une source de revenus pour le pays, et on estime qu'en 2008, les transferts représentaient jusqu'à $27 \%$ du PIB du Kirghizstan (ICG, 2010). Elles sont aussi un moyen pour le pouvoir politique de faire baisser la pression sociale et politique en « exportant» les mécontents potentiels.

Aussi, si l'on en croit tant les documents adoptés depuis 2005 que les déclarations officielles, il semblerait que le Kirghizstan ait pris le parti d'accompagner les migrations cherchant éventuellement à en transformer les caractéristiques afin d'en tirer le meilleur profit. Un des objectifs serait d'améliorer la formation afin de transformer le pays en " exportateur de main-d'œuvre qualifiée », puisqu'il est reconnu officiellement que « $90 \%$ des migrants travaillent au noir et... occupent en général des emplois mal payés, requérant peu de qualification (concierges, balayeurs, vendeurs sur les marchés, ouvriers sur les chantiers) » [Prog. migration Kyrg 2007-2010]. Ainsi en février 2010 Kubanychbek Issabekov, vice-speaker du Parlement déclarait que « si le Kirghizstan exporte sa force de travail, [...] il (lui) faut former les spécialistes dont les pays voisins ont besoin, la Russie par exemple » [Issabekov 2010].

L'autre enjeu qui se pose au Kirghizstan est celui d'une politique économique qui doit permettre de lutter contre le chômage, réinvestir les transferts ou l'argent des migrants, et enfin réintégrer les migrants qui reviennent dans le pays. Sans aborder les mesures économiques, la FIDH a souligné dans son rapport deux problèmes qui exigent des décisions politiques.

Le premier est celui de la corruption, un phénomène qui a des effets à différentes étapes du processus migratoire, à commencer par le moment de l'obtention d'un passeport $^{22}$. La corruption est aussi facteur d'émigration, dans la mesure où elle fait augmenter le coût d'accès à un certain nombre de biens et services (emplois dans l'administration, études dans les universités publiques). Elle crée des conditions d'insécurité pour l'activité économique et la petite entreprise, si bien que, selon une collaboratrice du Comité Kirghize des droits de l'homme «même si les gens savent qu'en Russie aussi, il $y$ a de la corruption, ils préfèrent aller là-bas, car au moins ils gagnent plus d'argent » [entretien Bishkek juin 2009]. Enfin, la corruption risque de freiner le réinvestissement par les citoyens kirghizes de l'argent gagné à l'extérieur.

22 En effet, selon les ONG kirghizes rencontrées par la mission FIDH en juin 2009, obtenir un passeport coutait entre 150 et 200 dollars US ; «même les personnes tout au bas de l'échelle au service des passeports sont couvertes d'or et de diamants ", s'était exclamé un de nos interlocuteurs pour montrer à quel point cette position était lucrative en raison de la corruption. Il existe d'ailleurs un programme de l'OIM « Kyrgyz Passport Modernization Programme », initié en 2003 et qui s'est transformé depuis en un grand programme anti-corruption et de capacity-building pour l'Agence nationale des passeports et le Département des passeports de la police kirghize (www.iom.int/jahia/Jahia/pid/507). 
Ce problème s'ajoute à un autre obstacle de type juridique, qui touche surtout les Kirghizes venant de Russie. En effet, en six ans, plus de 160000 Kirghizes ont reçu la citoyenneté russe, dans la mesure où les Kirghizes étaient éligibles pour une procédure simplifiée et que c'était souvent le seul moyen pour eux de travailler légalement, en particulier sur les marchés (en effet, depuis avril 2007, seuls les citoyens russes sont autorisés à commercer sur les marchés). Le système ne permettant pas la double citoyenneté, ils peuvent se voir confisquer leur passeport kirghize lorsqu'ils rentrent dans le pays [Issabekov 2009].

\section{Agir sur les conditions du départ}

À plusieurs reprises, les interlocuteurs rencontrés par la FIDH, ONG ou officiels, ont fait référence à « l'expérience des Philippines », dont $25 \%$ de la population travaille à l'étranger comme un exemple de politique migratoire. Ainsi, Aigul Ryskulova, lorsqu'elle était ministre du Travail, de l'Emploi et des Migrations, s'est rendue aux Philippines pour étudier leur expérience. À l'été 2009, la Banque mondiale a en effet organisé un voyage aux Philippines dans le cadre du «South-South Experience Exchange Between Practitioners (SEETF) » auquel ont participé plusieurs responsables kirghizes, programme comprenant de multiples rencontres avec des officiels philippins en charge des migrations $^{23}$. Quant à l'Organisation internationale des migrations (OIM), elle a fait appel comme expert principal de son programme sur les migrations en Asie centrale à Thomas Achacoso, un ancien diplomate et haut fonctionnaire philippin, qui a dirigé pendant de nombreuses années l'Agence pour l'Emploi à l'Étranger des Philippines (POEA). Lors de ses visites au Kirghizstan (comme d'ailleurs au Tadjikistan), il plaide pour que le pays adopte l'expérience des Philippines [Achacoso 2008]. Deux des éléments de la politique des Philippines sont généralement soulignés : l'information à destination des migrants avant le départ et le contrôle sur les agences de placement ${ }^{24}$.

En effet, de manière générale la méconnaissance par les migrants de leurs droits, mais aussi des formalités administratives auxquelles ils doivent se plier dans l'État d'accueil est redoutée tant par les autorités (qui ne veulent pas que leurs citoyens enfreignent la loi des États voisins) que par les ONG. Mal informés, les migrants se retrouveraient la proie d'intermédiaires indélicats, paieraient pour des services qui devraient être gratuits, acquerraient des fausses autorisations de séjour qui ne les protègent pas des

23 Le programme des rencontres ainsi que la composition de la délégation sont disponibles sur le site de la Banque mondiale, http://go.worldbank.org/66VYGPL5P0.

24 Le modèle philippin, donné en exemple par les organisations internationales, est en même temps une forme de gestion des migrations qui vise à contrôler strictement les migrants (interdiction de dépasser leur visa dans l'État d'emploi, possibilité d'être exclu du système, utilisation d'un système informatisé) et à donner une bonne image du « travailleur philippin » à l'étranger. Les États d'emploi trouvent un avantage certain à ce que les migrants soient ainsi strictement gérés par leur État d'origine. Enfin, le Comité sur les travailleurs migrants a regretté que cette politique semble surtout un moyen pour l'État d'exporter sa main d'œuvre, et a appelé les Philippines à « réexaminer sa politique de migration de la main-d'œuvre afin d'accorder la priorité aux droits de l'homme des travailleurs migrants » (Observations finales du Comité pour la protection des droits de tous les travailleurs migrants et des membres de leur famille PHILIPPINES, 22 mai 2009 $\mathrm{CMW} / \mathrm{C} / \mathrm{PHL} / \mathrm{CO} / 1)$. 
expulsions et seraient plus vulnérables à la corruption.

Aux Philippines, tous les migrants doivent obligatoirement assister à des séminaires d'information sur les lois des pays d'accueil et sur les ressources offertes par les consulats (Ruiz, 2008). Le gouvernement kirghize cherche lui aussi à informer et à former les migrants qui partent en utilisant différents canaux. Ainsi une « hotline » téléphonique a été mise en place avec le soutien de l'OIM, des centres d'information pour les migrants ont été ou vont être créés, et une série de clips télévisés va être diffusée [Ryskulova 2009].

Il n'est pas certain cependant que ces structures soient réellement utilisées : en 2006, 4500 personnes seulement avaient téléphoné à la hotline pour avoir des informations sur les modalités d'émigration ou d'installation dans un autre pays. Lors du séminaire organisé par la FIDH en novembre 2009, les ONG travaillant avec les migrants ont exprimé un certain scepticisme face à ces mesures. L'expérience montre que les migrants qui s'apprêtent à partir ne sont pas très intéressés par les informations sur les lois existantes, et ne considèrent pas forcément les organisations internationales, les ONG ou les autorités comme des sources fiables d'information; ils cherchent surtout à bénéficier des conseils et de l'expérience des migrants qui « ont réussi ».

L'autre élément clé de la politique migratoire philippine est le contrôle sur les agences de placement à l'étranger, afin d'éviter que les travailleurs migrants ne concluent de faux contrats, soient obligés de travailler illégalement ou victimes de trafic d'êtres humains ${ }^{25}$. Le système est contrôlé par la Philippine Overseas Employment Administration (POEA), qui note les agences privées et leur attribue des licences, mais joue aussi le rôle d'agence de recrutement.

Le gouvernement kirghize est conscient de l'importance des agences de recrutement : la Loi sur les migrations de travail extérieures de 2006 y consacre un chapitre entier. Le pays a annoncé aussi son intention de mettre en place une agence de recrutement étatique (Tsentr trudoustroistva za rubezhom) et préparait fin 2009 un projet de loi permettant de donner des licences à des agences privées [Ryskulova 2009].

Cependant, il semble illusoire d'espérer protéger les migrants en contrôlant les agences de recrutement alors que la très grande majorité des migrants n'a pas recours à ces agences. Selon une enquête menée par l'Institut d'étude de l'opinion publique El-Pikir seulement $8,5 \%$ des personnes interrogées ont trouvé un emploi grâce aux agences pour l'emploi, la majorité passant par des parents ou amis et $10 \%$ partant tout simplement « au hasard » [24.KG 23/01/2010]. En effet, la très grande majorité des migrants passe par des intermédiaires privés, le plus souvent des migrants originaires du même endroit qu'eux, qui ont déjà des contacts dans le pays d'accueil. Jouant le rôle de facilitateurs, ils sont utiles tant aux entreprises (leur offrant l'accès à une main-d'œuvre flexible) qu'à leurs compatriotes qu'ils aident à trouver du travail. Cependant, leur rôle les met en capacité

25 La question des agences est d'ailleurs évoquée par la Convention sur les Travailleurs Migrants qui donne la prééminence aux services d'État pour recruter des travailleurs dans un autre pays, et considère que des individus ou des agences privées ne devraient en être chargés que « sous réserve de l'autorisation, de l'approbation et du contrôle des organes officiels des États partis intéressés » (art. 66). 
d'exploiter les travailleurs migrants (prélèvement d'un pourcentage parfois exorbitant sur les salaires, confiscation de passeport, revente à des employeurs, etc.), et ceux-ci hésitent souvent à dénoncer des intermédiaires dont ils ont besoin pour trouver du travail.

L'objectif, pour le gouvernement kirghize, devrait donc être également de poursuivre les intermédiaires privés quand ceux-ci sont impliqués dans des affaires de trafic d'êtres humains ou de travail forcé - il ne semble pas cependant que la procurature, chargée de ces poursuites, y mette beaucoup d'énergie. Rencontré en novembre 2009, le Procureur-Général adjoint a expliqué que c'était le ministère des Affaires étrangères qui avait les informations sur le nombre d'affaires ouvertes pour trafic d'êtres humains, et a rejeté la responsabilité de lutter contre le travail forcé sur le « nouveau ministère du Travail » [Nasiza 2009].

\section{Protéger les migrants résidant à l'étranger}

Selon la Convention sur les travailleurs migrants, les États d'émigration ont une responsabilité envers leurs ressortissants (art. 7). Dans les faits, la défense de l'intérêt de leurs citoyens à l'étranger peut passer par des négociations politiques pour obtenir un quota « national » avantageux, par la conclusion d'accords bilatéraux ad hoc, elle peut impliquer l'utilisation des ressources du pays (l'eau dans le cas du Kirghizstan) et une utilisation des différentes arènes de négociation. Le Kirghizstan se targue ainsi d'avoir joué un rôle dans l'adoption de mesures contre l'arbitraire policier dans le cadre de la Convention de la CEI sur la protection des travailleurs migrants, et d'être le premier pays à lancer l'expérience pilote de la « carte de voyage du migrant » qui offrirait une protection médicale dans le cadre de la convention de Shanghai [Ryskulova 2009]. Néanmoins, à l'instar du Kirghizstan, les États d'émigration qui cherchent à défendre leurs migrants sont rarement en position de force pour le faire, puisqu'ils sont le plus souvent en situation de dépendance et d'infériorité économique face aux États d'emploi de leurs ressortissants. Les aléas des relations avec le Kazakhstan rappellent ainsi que même avec une politique pro-active, les ressources des États d'émigration restent limitées.

\section{Le rôle du réseau consulaire}

Selon l'article 23 de la Convention sur les travailleurs migrants, «les travailleurs migrants et les membres de leur famille ont le droit d'avoir recours à la protection et à l'assistance des autorités consulaires ou diplomatiques de leur État d'origine [...] en particulier, en cas d'expulsion ». L'article 16 de la loi « sur les migrations extérieures du Kirghizstan » prévoit aussi que les travailleurs migrants ont le droit de "s'adresser aux services diplomatiques et consulaires, situés dans l'État d'embauche, afin de défendre leurs droits et libertés à un travail ».

Cette protection peut commencer en amont dans la définition du type de passeports qui vont servir à sortir du pays. Au Kirghizstan, il a pu exister jusqu'à quatre types de passeports, dont certains étaient remplis à la main, à l'encre : il suffisait que le passeport tombe à l'eau pour qu'il soit illisible et que son possesseur soit en situation délicate ; en novembre 2009 le gouvernement avait d'ailleurs l'intention d'introduire des passeports 
biométriques [Ryskulova 2009].

La protection dépend ensuite de la capacité et de la volonté des consulats d'assurer gratuitement un certain nombre de services : délivrance de documents et attestations, soutien juridique dans les contentieux de travail ou lors des expulsions. Outre son réseau consulaire, le gouvernement kirghize a ouvert une représentation du Comité des Migrations et de l'Emploi au consulat d'Almaty, ainsi qu'un Centre d'information au Parc des expositions (VDNKh) de Moscou. Les résultats restent encore à évaluer, même si les autorités n'hésitent pas à vanter leurs succès. Ainsi Aigul Ryskulova, responsable du Comité des migrations et de l'Emploi, a déclaré en juin 2009 que «lorsque nous avons ouvert nos représentations en Russie, 13000 Kirghizes avaient des permis de travail maintenant, ils sont 330 000. Ces statistiques montrent le résultat de notre travail ». S’il y a certainement eu une augmentation du nombre de Kirghizes travaillant légalement en Russie, c'est sans doute surtout un effet des réformes de la politique migratoire russe, qui ont facilité pour tous les migrants l'obtention d'un permis de travail.

Enfin, le réseau consulaire est censé assurer la participation des migrants aux affaires publiques de leur État, en conformité avec l'article 41 de la Convention des Travailleurs migrants. Lors du référendum du 27 juin 2010, le gouvernement avait ouvert dix bureaux de vote en Russie et trois au Kazakhstan (deux à Almaty et un à Astana). La ministre par intérim Bodosh Mamyrova notait d'ailleurs à ce propos que de nombreux migrants en Russie avaient peur d'aller voter, craignant d'être déportés, et que le Service Fédéral russe des Migrations allait mener une campagne d'information pour assurer les migrants irréguliers qu'ils ne seraient ni arrêtés ni déportés à ce moment-là [Mamyrova 2010].

\section{Kazakhstan/Kirghizstan}

Alors que le nombre de migrants kirghizes travaillant au Kazakhstan est estimé entre 60000 et 100 000, les aléas des relations entre Kazakhstan et Kirghizstan illustrent à la fois le type de politique qui peut être mené pour sécuriser la situation des migrants, mais aussi les limites de cette politique, qui n'échappe pas aux intérêts et aux jeux politiques internes.

Plusieurs accords bilatéraux ont en effet été signés entre le Kirghizstan et le Kazakhstan, le premier étant l'accord sur les travailleurs migrants employés dans l'agriculture dans les régions frontalières signé en 2002. Selon l'Ombudsman kirghize Tursunbek Akun, c'est à son prédécesseur que revient le mérite d'avoir attiré l'attention sur la question des travailleurs agricoles travaillant dans les plantations de tabac - un scandale qui a justement abouti à la signature d'un accord ad hoc avec le Kazakhstan sur ces questions. De fait, le problème de l'exploitation des migrants dans les plantations de tabac n'est toujours pas réglé. Dans un rapport publié en juillet 2010, Human Rights Watch dénonce le fait que de nombreux travailleurs migrants y ont été escroqués et exploités, certains se retrouvant en situation de travail forcé, et souligne la responsabilité du groupe Philip Morris, avec lequel les propriétaires fermiers qui emploient des saisonniers sont sous contrat (HRW, 2010). 
En plus d'un accord de 2006 sur les droits des travailleurs kirghizes travaillant temporairement au Kazakhstan, les deux pays ont conclu en juin 2009 un accord permettant aux petits entrepreneurs individuels d'obtenir des patentes et de travailler sans avoir besoin de former des personnalités juridiques. Cet accord visait essentiellement à faciliter la situation des citoyens kirghizes faisant du commerce et travaillant sur les marchés.

Cependant, l'accord n'est pas entré en vigueur. La ministre du Travail Aigul Ryskulova l'expliquait en février 2010 par l'entrée du Kazakhstan dans une union douanière avec la Russie qui créait un système unique de circulation des biens et des entrepreneurs, et par l'adoption, postérieure à l'accord, d'une loi « sur l'entrepreneuriat privé » qui prévoit que seuls les citoyens kazakhs et les Oralman peuvent travailler comme entrepreneurs individuels, les étrangers étant obligés de former des personnes morales [Ryskulova 2010].

Le retour en arrière des autorités kazakhes sur ce point semble s'être accompagné d'un durcissement de la politique migratoire. En février 2010, une centaine de personnes, essentiellement des migrants travaillant sur les marchés d'Almaty, se sont réunies sur la place centrale de Bichkek pour se plaindre de l'augmentation des expulsions et exiger des autorités qu'elles entament des négociations avec leurs homologues kazakhes. La ministre du Travail est d'ailleurs sortie pour écouter leurs revendications - une réaction à signaler quand on sait que depuis des mois, les manifestations à Bichkek étaient systématiquement interdites et réprimées. Cet épisode montre donc aussi la capacité des migrants à se transformer en force politique interne, capacité qu'illustre la rapide montée en puissance du parti Zamandash créé par des migrants, formé de plusieurs entrepreneurs ayant acquis leur situation en Russie et qui a finalement été exclu des élections de 2007 pour des raisons douteuses (ICG, 2010).

Ce raidissement du Kazakhstan, qui s'inscrit dans une volonté affichée de durcir les conditions d'entrée des migrants dans le pays, s'est encore aggravé après les révoltes qui ont mené à la chute du président Bakiev. En avril 2010, le Kazakhstan a fermé sa frontière avec son voisin pour plusieurs jours, officiellement pour empêcher des armes d'entrer dans le pays et pour empêcher des « criminels » de se réfugier au Kazakhstan. Les autorités kirghizes ont rapporté des cas de rafles et de déportations de leurs citoyens résidant à Almaty, et il semblerait même que dans certains cas les parents aient été déportés sans leurs enfants qui se trouvaient à l'école [Mintrud Kyrg mai 2010]. Enfin, après les émeutes meurtrières qui ont eu lieu au sud du Kirghizstan, les autorités kirghizes ont affirmé que la police des frontières du Kazakhstan avait fermé la frontière de manière unilatérale et "interdisait l'entrée dans le pays de citoyens kirghizes d'origine ouzbèke » [KT.Kz 16/06/2010].

Ce dernier exemple nous permet de rappeler à quel point les migrations sont vues, par les États, comme des menaces à leur sécurité. L'appartenance du Kazakhstan et du Kirghizstan à des systèmes collectifs qui définissent explicitement les migrations « illégales » comme une menace aggrave sans doute encore cette tendance, mais celle-ci n'est pas propre aux États de la région. La question des migrations reste, en effet, comme celle de l'anti-terrorisme, un des domaines où ceux qui se disent démocratiques enfreignent avec le plus de facilité leurs propres principes. À cet égard, la réticence du Kazakhstan à 
ratifier la Convention sur les travailleurs migrants n'est absolument pas une exception, la majorité des États européens refusant toujours de le faire. La priorité est donnée à l'utilité économique des migrants pour l'État d'accueil, et non à la garantie de leurs droits.

De même, les effets de la politique migratoire du Kazakhstan en termes de migrations irrégulières peuvent être vus comme une illustration du cercle vicieux dans lequel sont souvent enfermés les migrants : les États d'accueil leur reprochent d'être « illégaux » et leur enjoignent d'être en situation régulière alors même qu'ils ne leur donnent pas les moyens de régulariser leur situation.

Enfin, l'étude des politiques migratoires kazakhes et kirghizes rappelle que les politiques migratoires impliquent non seulement l'État d'emploi, mais aussi l'État d'origine des migrants, qui a des responsabilités et une marge de manœuvre, même limitée. Elles impliquent également les organisations internationales, qui à l'instar de la Banque Mondiale ou de l'OIM, cherchent à diffuser ce qu'elles définissent comme des «bonnes pratiques » en termes de migrations. La diffusion de ces pratiques et de ces règles reste cependant illusoire si elles ne sont pas investies et appropriées par les migrants eux-mêmes, migrants dont les stratégies peuvent ne pas coïncider avec les buts et les règles fixés par ces organisations. C'est là une des raisons pour lesquelles, du point de vue des organisations de défense des droits de l'homme, il ne s'agit pas tant de «protéger» les migrants que de leur garantir la possibilité de défendre eux-mêmes leurs droits.

\section{Références bibliographiques et documentaires}

DOLOTKELDIEVA Asel (2010) Les migrants kirghizes à Moscou : politiques publiques, stratégies migratoires et réseaux associatifs, in Marlène Laruelle Éd., Dynamiques migratoires et changements sociétaux en Asie centrale, Paris, Pétra, pp. 123-145.

FAUVE Adrien (2010) La question des droits de l'homme au Kazakhstan : discours officiel et réalités des faits sur fond de présidence de l'OSCE, dossier du CERI, http://www.ceri-sciencespo.com.

FIDH (2009) Kazakhstan / Kyrgyzstan : Exploitation of Migrant Workers, Protection Denied to Asylum Seekers, Paris, 92 p, http://www.fidh.org/IMG/pdf/Kazakhstan530a.pdf (disponible en anglais et en russe).

FIDH (2010) Migrations internationales : Travailleurs migrants, demandeurs d'asile et réfugiés en Europe de l'Est, Asie centrale et Caucase du Sud (Séminaire régional de la FIDH), 32 p, www. fidh.org/Migrations-internationales-Travailleurs-migrants (disponible en français, en anglais et en russe).

HRW (2010) “Hellish Work” Exploitation of Migrant Tobacco Workers in Kazakhstan, New York, 115 p., http://www.hrw.org/en/reports/2010/07/14/hellish-work-0.

ICG (2010) Central Asia : Migrants and the Economic Crisis, Asia Report, 183, http://www.crisisgroup.org/en/regions/asia/central-asia/183-central-asia-migrants-and-the-economic-crisis.aspx.

ILO (2008) Rights of Migrant Workers in Kazakhstan : National Legislation, International Standards and Practices, Almaty, $55 \mathrm{p}$.

LARUELLE Marlène (2010) Le Kazakhstan, nouveau carrefour migratoire d'Asie centrale, in Marlène Laruelle $\dot{E} d$., Dynamiques migratoires et changements sociétaux en Asie centrale, Paris, Pétra, pp. 77-98.

OIM (2009) Obzor pravovykh problem trudiashikhsa-migrantov v respublilke Kazakhstan (po itogam deiatelnosti NPO-Partnerov v 2007-2009) (Analyse des problèmes juridiques des 
travailleurs migrants au Kazakhstan (basée sur l'activité des ONG partenaires en 2007-2009), Almaty, 20 p.

OIT (2005) Une alliance mondiale contre le travail forcé, Rapport global en vertu du suivi de la Déclaration de l'OIT relative aux principes et droits fondamentaux au travail, Conférence internationale du travail, 93 ${ }^{\mathrm{eme}}$ session, http://www.ilo.org/public/french/standards/relm/ilc/ilc93/ pdf/rep-i-b.pdf.

RUIZ Neil G. (2008) Managing Migration: Lessons from the Philippines, World Bank, Migration and Development Brief, 11 août 2008.

SANA Syzim (2009) Monitoring po proektu 'Okazanie pravovoi pomoshi trudovym migrantam's 01.11.2008g. po 31.05.2009g (Monitoring du projet d'aide juridique aux travailleurs migrants du 01/11/2008 au 31/05/2008), http://migrant-help.org/pages/120_monitoring/main.htm.

THOREZ Julien (2007) Itinéraires du déracinement. L'essor des migrations de travail entre l'Asie centrale et la Russie, Espaces, populations et sociétés, 1, pp. 59-71.

UNIFEM (2009) Needs Assessment of Women Labour-Migrants, Central Asia and Russia, (Red E. Turukanova), 70 p, http://www.unifemcis.org/img/database/175.pdf.

\section{Articles de journaux}

[24.kg, 23/01/2010] : «El-Pikir : V Kyrgyzstane naibolee zazhitochnye trudovye migranty prozhivaiut v Oshskoi i Batkenskoi oblastiakh (El Pikir : au Kirghizstan les migrants les plus aisés vivent dans les régions d'Osh et Batken) », 24.KG, 23/01/2010, http://24.kg/community/68250laquoyel-pikirraquo-v-Kirghizstane-naibolee.html.

[24.kg, 28/01/2010] : Pravitel'stvo Kyrgyzstana opredelilo velichinu kvoty na privlechenie inostrannoi trudovoi sily na 2010 god (Le gouvernement du Kirghizistan a défini les quotas de main-d'œuvre étrangère pour 2010) ", Dépêche, 24.KG, 28/01/2010, http://www.24.kg/ community/64842-o-zhenshhina-tyhellip.html.

[Abdykalova 2010] : « V Kazakhstane predlagaiut vvesti stranovye, otraslevye kvoty inostrannoj rabochei sily (Le Kazakhstan propose d'introduire des quotas de main-d'œuvre étrangère par pays et par secteur) », Interview de la ministre du Travail et de la Protection sociale du Kazakhstan Gulshara Abdykalova, 18/01/2010, http://www.zpress.kg/news/news_only/10/14327.py.

[Achacoso 2008] : «T.Achakoso : Protsess migratsii v Kyrgyzstane ostanovit' nevozmozhno, dazhe esli vozvesti vokrug gosudarstva vysokie steny (T. Achakoso : le processus de migration au Kirghizstan ne peut être stoppé, même en érigeant des hauts murs autour du pays) », Interview au journal KG, 28/10/2008, www.centrasia.ru/newsA.php?st=1225178460.

[Isabekov 2010] : « Kubanychbek Isabekov: Esli Kyrgyzstan eksportiruet rabochuiu silu, to neobkhodimo obespechit' nuzhdaiushiesia strany kvalifitsirovannymi spetsialistami (Kubanychbek Isabekov : si le Kirghizistan exporte sa force de travail, alors il est indispensable d'assurer au pays qui en a besoin des spécialistes qualifiés) », 24.KG, 10/02/2010, http://www.24.kg/ community/69136-kubanychbek-isabekov-esli-Kirghizstan.html.

[Kravets 2009] : KRAVETS Pavel (10/03/2009), « Uzbekskie migranty v Kazakhstane, Trudnosti Perehoda (Les migrants ouzbeks au Kazakhstan : les difficultés du passage) », ferghana.ru, http://www.ferghana.ru/article.php?id=6088.

[KT.Kz 16/06/2010] : « Kazahstan zakryl granicy s Uzbekistanom i vvel zapret na vezd v stranu grazhdan KR uzbekskoi natsional'nosti (Le Kazakhstan a fermé la frontière et interdit l'entrée dans le pays de citoyens kirghizes de nationalité ouzbèke) », KT.Kz, 16/06/2010, http://kt.kz/?1 ang $=$ rus\&uin $=1253258757 \&$ chapter $=1153519523$.

[Mamyrova 2010] : « Bodosh Mamyrova: Trudovye migranty iz Kyrgyzstana v Rossii ne khotiat golosovat' na referendume, opasaias', chto ikh deportiruiut (Bodosh Mamyrova : les travailleurs migrants kirghizes en Russie ne veulent pas voter lors du référendum, craignant qu'on les déporte) », Dépêche, 24.KG, 23/06/2010, http://www.24kg.org/politic/77309-bodosh-mamyrova-trudovye-migranty-iz-Kirghizstana.html. 
[Ryskulova 2010] : «Aigul' Ryskulova: Otchasti vlasti Kazahstana pravy v zakonnosti deportatsii migrantov iz Kyrgyzstana (Aigul Ryskulova : les pouvoirs du Kazakhstan ont en partie raison sur la légalité de la déportation des migrants kirghizes) ", Dépêche, 24.KG, 12/02/2010, http:// www.24.kg/news-stall/69304-ajgul-ryskulova-otchasti-vlasti-kazaxstana-pravy.html.

[Tashimov 2009] : TASHIMOV Tulin (9/03/2009), « Chuzhie zdes' ne hodiat (Il n’y a pas d'étrangers ici) », Ekspert Kazakhstan, 9 (205), http://www.expert.ru/printissues/kazakhstan/2009/09/ trud/.

\section{Sites Internet consultés}

www.centrasia.ru

www.zpress.kg : Zamandash press

www.rferl.org : Radio Free Europe / Radio Liberty

http://www.24.kg/: Agence de presse kirghize

http://migrant.ferghana.ru/ : Site d'information spécialisé sur les migrations d'Asie centrale

http://www.1.ca-news.org/ : AKI Press (Kirghizstan)

www.diapazon.kz : Journal en ligne, Kazakhstan

\section{Entretiens de la mission FIDH}

[Abysheva 2009] : avec Khadyshat Abysheva, directrice de l'ONG Sana Syzim, Chymkent, 16/06/2009.

[Issabekov 2009] : avec K. Issabekov, vice-speaker du Parlement, Bishkek, 20/06/2009.

[Kaliuzhin 2009] : avec Vyacheslav Kaliuzhin, Centre des droits de l'homme du bureau de l'Ombudsman, Astana, 11/06/2009.

[Nasiza 2009] : avec Sumar Nasiza, vice-procureur général, Bishkek, 30/11/2009.

[Nusupova-Lepekha 2010] : avec Asel Nusupova, Vice-ministre du Travail et de la Protection sociale et Igor Lepekha, Lieutenant, vice-président du Comité de la Police administrative au ministère de l'Intérieur, Genève, 11/05/2010 (lors de la session du Comité des droits économiques, sociaux et culturels de l'ONU).

[Nusupova-Nurseitov 2009] : avec Assel Nusupova, vice-ministre du Travail et de la protection sociale et Amandjur Nurseitov, Directeur du Département de l'Emploi et des Migrations, Astana, 12/06/2009.

[Ryskulova 2009] : avec Aigul Ryskulova, responsable du Comité d'État pour les Migrations et l'Emploi, Bishkek, 19/06/2009.

[Ryskulova 2009] : avec Aigul Ryskulova, ministre du Travail, de l'Emploi et des Migrations, Bishkek, 11/2009.

\section{Documents officiels et textes juridiques nationaux (entre crochets dans le texte)}

\section{Kazakhstan}

[Amnistie Kaz 2006] : Zakon Respubliki Kazakhstan «Ob amnistii v sviazi s legalizatsiei nezakonnykh trudovykh immigrantov» (Loi de la République du Kazakhstan d'amnistie en lien avec la légalisation des travailleurs migrants illégaux), 4/07/2006, site du ministère de l'Intérieur http:// www.mvd.kz/index.php?p=razdel_more\&id5=259\&id1 $=3$.

[Conception pol. mig Kaz. 2007-2015] : Kontseptsia migratsionnoi politiki Respubliki Kazakhstana na 2007-2015 gody (Conception de la politique migratoire du Kazakhstan pour 2007-2015), http://www.enbek.gov.kz/law/lawdetail.php?recordID=364. 
[Duisenova 2009] : Thèses de la secrétaire du ministère du Travail T. Duisenova présentées à un séminaire international sur les meilleures pratiques migratoires dans le domaine des migrations (organisé conjointement par le Parlement kazakh, OIT, OSCE, OIM, EU), 29/10/2009, http:// www.zakon.kz/151653-v-mazhilise-prezentovan-novyjj.html.

[Loi migration Kaz 1997] : Zakon Respubliki Kazakhstan O migratsii naselenia ot 13 dekabria 1997 goda (Loi de la République du Kazakhsran sur la migration de la population du 13/12/1997), http://www.antropotok.archipelag.ru/text/a081.htm.

[Mintruda mai 2010] : Document interne du ministère du Travail et de la Protection sociale du Kazakhstan remis par la vice-ministre A. Nusupova à la FIDH (11/05/2010).

[Nurly Kosh] : Décret du gouvernement $\mathrm{n}^{\circ} 1126$ du 2 décembre 2008, présentation officielle sur http://www.zakon.kz/130010-nurly-kosh-programma-dejjstvijj.html.

\section{Kirghizstan}

[Conception pol. mig. Kyrg 2005-2010] : «Kontsetpsia gosudarstvennoi migracionnoi politiki Kyrgyzskij Respubliki do 2010 goda (Conception de la politique migratoire de l'État pour 20052010) », http://www.government.gov.kg/index.php?option=com_content\&task=view\&id=67.

[Loi Migration Kyrg 2006] : "Zakon o vneshnei trudovoij migratsii (Loi sur les migrations de travail extérieures) », 13/01/2006, http://mz.kg/ru/law_labourmigration/.

[Mintrud Kyrg mai 2010] : «Po situatsii o polozhenii kyrgyzskikh migrantov osushestvliaiushikh predprinimatel'skuiu deiatel'nost' v torgovo-rynochnykh kompleksakh Kazakhstana (Sur la situation des migrants kirghizes travaillant comme entrepreneurs dans les marchés et centres commerciaux du Kazakhstan) ", site officiel du ministère de 1'Emploi, du Travail et des Migrations http://www.mz.kg/ru/news/217/.

[Prog. migration Kyrg 2007-2010] : «O Gosudarstvennoi programme Kyrgyzskoj Respubliki po regulirovaniu migracionnykh protsessov na 2007-2010 gody (sur le Programme d'État de la République Kirghize pour la régulation des processus migratoires en 2007-2010) », adopté par le gouvernement kirghize le 25/09/2007, Accès octobre 2009, http://rus.gov.kg/ index.php?option $=$ com_content\&task=view\&id $=1296$.

\section{Textes et conventions internationales (entre crochets dans le texte)}

[C143 OIT] : Convention 143 de l'OIT sur les travailleurs migrants (dispositions complémentaires), http://www.ilo.org/ilolex/cgi-lex/convdf.pl?C143.

[C97 OIT] : Convention 97 de l'OIT concernant les travailleurs migrants (révisée), 1949, http:// www.ilo.org/ilolex/cgi-lex/convdf.pl?C097.

[CESCR Kaz 2010] : "Concluding Observations of the Committee on Economic, Social and Cultural Rights. Kazakhstan », 21/05/2010, E/C.12/KAZ/CO/1, http://www2.ohchr.org/english/ bodies/cescr/cescrs44.htm.

[Charte CSTO 2002] : Charter of the Collective Security Treaty Organisation, Chisinau, 7/10/2002, http://untreaty.un.org/unts/144078_158780/5/9/13289.pdf.

[Convention CEI sur les travailleurs migrants] : « Convention sur le Statut légal des travailleurs migrants et des membres de leurs familles - des États membres de la CEI », http://www.cis. minsk.by/webnpa/text.aspx?RN=N00800671.

[Convention sur les travailleurs migrants, CTM] : Convention internationale sur la protection des droits de tous les travailleurs migrants et des membres de leur famille, 1990, http://www2.ohchr. org/french/law/cmw.htm.

[HRC Kyrg 2000] : Concluding observations of the Human Rights Committee : Kyrgyzstan, 24/07/2000, CCPR/CO/69/KGZ, http://www.unhchr.ch/tbs/doc.nsf/(Symbol)/CCPR.CO.69. KGZ.En?Opendocument.

[Traité de Shanghai 2007] : Traité sur l'amitié, le bon voisinage et la coopération entre les États membres de l'Organisation de coopération de Shanghai, signé en 2007, http://www.sectsco.org/ EN/show.asp?id=71 (en anglais). 


\title{
Kazakhstan, Kirghizstan : les politiques migratoires au regard des droits de l'homme
}

\author{
Amandine REGAMEY et Katherine BOOTH
}

\begin{abstract}
Basé sur une enquête et un rapport de la Fédération internationale des droits de l'homme de 2009, cet article évalue la politique migratoire du Kazakhstan et du Kirghizstan au regard des normes internationales qui définissent les droits des travailleurs migrants (notamment la Convention sur les travailleurs migrants de l'ONU et les conventions de l'OIT, qui entrent parfois en conflit avec des accords régionaux ou bilatéraux). Pays d'immigration, le Kazakhstan cherche avant tout à attirer une main-d'œuvre qualifiée, qui doit être remplacée à terme par des spécialistes kazakhes. Le système de quota et des règles très contraignantes limitent les possibilités pour les migrants de travailler légalement et condamnent à l'irrégularité la grande majorité d'entre eux. Maintenus dans une situation juridique et matérielle précaire, ceux-ci sont les plus susceptibles d'être victimes du travail forcé. Le cas du Kirghizstan, pays d'émigration, rappelle les responsabilités, mais aussi les difficultés d'un État de départ pour protéger ses ressortissants à l'étranger - comme le montrent les aléas des relations avec le Kazakhstan. Il permet aussi d'illustrer la manière dont les pratiques de gestion des migrations sont importées (en l'occurrence des Philippines), avec l'aide des organisations internationales et les limites de cette démarche.
\end{abstract}

\section{Kazakhstan, Kirgizstan: Migrations Policies in the Eyes of Human Rights Question}

\author{
Amandine REGAMEY and Katherine BOOTH
}

Drawing upon the findings of an investigation conducted by the International Federation for Human Rights in 2009, this article analyses the migration policies of Kazakhstan and Kyrgyzstan in light of the international standards defining the human rights of migrant workers and against a background of sometimes conflicting regional and bilateral commitments. As a country of immigration, Kazakhstan seeks to attract qualified migrant workers on a temporary basis, whilst training Kazakh nationals to replace them in the long-term. The restrictive and complex quota system severely limits the possibilities for migrants to work legally and the vast majority of migrant workers thus find themselves in an irregular situation. As a result of legal insecurity and material hardships, such migrants are vulnerable to abuse including forced labour. The example of Kyrgyzstan illustrates the responsibilities of a state of departure to protect its nationals abroad and the challenges faced. The article also examines how, with the support of international organisations, models for "managing" migration (such as that developed in the Philippines) are imported and explores the limitations of this approach. 


\section{Kazajstán, Kirguistán: las políticas migratorias con respecto a los derechos humanos}

\section{Amandine REGAMEY y Katherine BOOTH}

Basado en un informe de la Federación internacional de los derechos humanos del año 2009, este artículo evalúa la política migratoria de Kazajstán y de Kirguistán con respecto a las normas internacionales que definen los derechos de los trabajadores migrantes (entre otros la Convención sobre los trabajadores migrantes de la ONU y las convenciones de la OIT que a veces entran en conflicto con algunos acuerdos regionales o bilaterales). País de inmigrantes, Kazajstán busca ante todo atraer mano de obra cualificada que debería ser, a largo plazo, substituida por especialistas kazajos. El sistema de cuotas y las normas excesivamente restrictivas limitan las posibilidades de los inmigrantes de trabajar legalmente y obligan la gran mayoría de ellos a la irregularidad. Mantenidos en una situación jurídica y material precaria, son estos los que serán con más probabilidad víctimas de trabajos forzados. El caso de Kirguistán en cambio, país de emigrantes, recuerda las responsabilidades pero también las dificultades a las cuales un Estado que exporta su mano de obra se enfrenta para proteger a sus ciudadanos en el extranjero - como lo demuestran los altibajos en sus relaciones con Kazajstán. Finalmente, este caso permite también ilustrar de un lado como las prácticas de gestión de las migraciones pueden ser importadas (en este caso de Filipinas) con el apoyo de las organizaciones internacionales y de otro lado cuáles son las limitaciones que este enfoque migratorio origina. 


\title{
Revue européenne des migrations internationales
}

vol. $26-\mathrm{n}^{\circ} 3$ (2010)

Migrations en Asie centrale et au Caucase

Sergey Rumyansev

\section{Post-Soviet Nation State as a Sponsor of Construction of the Ethno-National Diaspora: Azeri's Case}

\author{
Avertissement \\ Le contenu de ce site relève de la législation française sur la propriété intellectuelle et est la propriété exclusive de \\ l'éditeur. \\ Les œuvres figurant sur ce site peuvent être consultées et reproduites sur un support papier ou numérique sous \\ réserve qu'elles soient strictement réservées à un usage soit personnel, soit scientifique ou pédagogique excluant \\ toute exploitation commerciale. La reproduction devra obligatoirement mentionner l'éditeur, le nom de la revue, \\ l'auteur et la référence du document. \\ Toute autre reproduction est interdite sauf accord préalable de l'éditeur, en dehors des cas prévus par la législation \\ en vigueur en France.
}

\section{revues.org}

Revues.org est un portail de revues en sciences humaines et sociales développé par le Cléo, Centre pour l'édition électronique ouverte (CNRS, EHESS, UP, UAPV).

\section{Référence électronique}

Sergey Rumyansev, « Post-Soviet Nation State as a Sponsor of Construction of the Ethno-National Diaspora: Azeri's Case », Revue européenne des migrations internationales [En ligne], vol. $26-n^{\circ} 3$ | 2010, mis en ligne le 01 décembre 2013, consulté le 03 janvier 2014. URL : http://remi.revues.org/5218 ; DOI : 10.4000/remi.5218

Éditeur : Université de Poitiers

http://remi.revues.org

http://www.revues.org

Document accessible en ligne sur : http://remi.revues.org/5218

Ce document est le fac-similé de l'édition papier.

(c) Université de Poitiers 


\title{
Post-Soviet Nation State as a Sponsor of Construction of the Ethno-National Diaspora: Azeri's Case
}

\author{
Sergey RUMYANSEV ${ }^{1}$
}

\section{INTRODUCTION}

n 18.12.2008 most Azerbaijani media circulated reports saying that the
Charter of Solidarity of Azeris of the World had finally been adopted at a session of the Coordinating Council of Azeris of the World held in Baku. In the past few years officials of various ranks have been saying that such a document is necessary and work on it is under way. It is symbolic that this session was held in Baku in the State Committee of the Azerbaijani Republic for Work with the Diaspora with the immediate participation of its director Nazim Ibrahimov. As it turned out later, journalists were somewhat too quick to report that. Only a preliminary version was adopted, work on which is still under way. However, something else is important too. The preparation of such a Charter in the capital of independent Azerbaijan is, in my view, a demonstration of how an independent state not only sponsors the process of construction of diaspora but also tries to control it. The specifics of the diaspora policy being conducted by the Azerbaijani authorities may serve as a good illustration to the thesis by Tishkov (2003: 41) which says that: "The so-called nation state and not ethnic commonality is key to diaspora formation".

The policy of constructing an Azeri ethno-national diaspora started to be intensively implemented in the early $21^{\text {st }}$ century. However, the formation of this purposeful policy was preceded by various events that demonstrated a constantly increasing interest on the part of the state in ethnic Azeris living in various countries of the world.

1 Research Fellow at the Institute of Philosophy, Sociology and Law of the National Academy of Sciences of Azerbaijan. Head of the independent social research group "Novator" (Baku). PostDoctoral research fellow at the Institute for European Ethnology, Humboldt-University Berlin; sergnovator@yandex.ru 
This article, presenting the results of the research into the process of formation of diaspora politics in Azerbaijan and also analyses the aims and specifics of the mechanisms of its implementation, has been prepared primarily on the basis of field materials collected during the implementation of a research project focused on "Diaspora Politics" in postsoviet Azerbaijan supported by the Caucasus Resource Research Center. In addition, the specifics of the influence of the state diaspora politics on the activity of expatriates is also analyzed on the basis of some projects which the author implemented with the support of various foundations, as Heinrich Böll Stiftung, Centre for Independent Social Research and Smolnyy Collegium of St Petersburg or Georg-Eckert-Instituts für Internationale Schulbuchforschung, within four years in Georgia, Russia, and Germany.

\section{RESEARCH METHODOLOGY}

The research methods were participant observation, problem-oriented semiformalized interviews, and critical discourse analyses of media materials, speeches and interviews of officials of various ranks and public figures, various official decrees... Problem-oriented semi-formalized interviews were conducted in Azerbaijan in 2008-09, with officials whose main task is to implement the diaspora project, and activists of organizations created in the course of the implementation of the diaspora project both in Azerbaijan and abroad. Another batch of material I collected using the method of participant observation of various events held in the course of the implementation of diaspora politics (conferences, meetings, etc.).

A major research method was also critical discourse analysis of a considerable body of texts which represent the official version of the meaning and results of the diaspora policy being conducted (Fairclough, 1995). These are the texts of numerous addresses by the presidents of Azerbaijan Heydar Aliyev (1993-2003) and Ilham Aliyev (2003-to date) to Azeris in emigration; numerous interviews published in Azerbaijani media in which both presidents and also officials of various ranks and activists of diaspora organizations touch upon various issues of diaspora politics' speeches by the Azerbaijani presidents, officials, intellectuals and also activists of diaspora organizations at two congresses of Azeris of the world held in Baku; news reports on the activity of "Azeris in the diaspora", and various documents which reflect the diaspora policy of Azerbaijan's political regime.

\section{“AZERI DIASPORA” AS A POLITICAL PROJECT}

First of all, I should stress that the criteria that are the most known in academic discourse, which are used to identify the phenomenon of (ethno-national) diaspora and are proposed by leading specialists, cannot by far be always relevant in the case of research into social networks and ethnic organizations of Azeris in emigration. Thus, Safran (1991) singles out six main characteristics of such diasporas: dispersion from the original "centre", to at least two "periferal" places; presence of memory or a myth about homeland; the belief that members of diaspora will not be completely accepted by new country; ideas about homeland as a place of inevitable return; commitment to support or restore homeland; and presence of group solidarity and feeling of connection to homeland. For Azeris in 
emigration such centres from where dispersion stemmed are not one but several countries with which they actually identify themselves. Integration into the host community is also not perceived unequivocally, depending on from what countries emigration came and also what country is the host one. For example, in Russia for most emigrants of the soviet wave no problems with integration arise, while work migrants of the post-soviet wave often face serous difficulties or consider their stay in Russia only as temporary. The criteria of belief in inevitable return to motherland and the feeling of connection to it are also not relevant considering the fact that there is not one such motherland but for most Iranian or Turkish Azeris the Azerbaijani Republic is not perceived as one homeland for all. Finally, group solidarity looks like the most problematic one. For Russian speaking Azeris group solidarity is defined not through ethnicity but through shared soviet socialization and language. The borders between the so-called Iranian and Turkish Azeris and natives of the Azerbaijan Republic itself also preserve their firmness.

Cohen (1996: 515) expands the range of the criteria proposed by Safran. As a result, the number of such "common features" of diaspora goes up to nine. They include: "the expansion from a homeland in a search of work, in pursuit of trade to further colonial ambitions"; " a strong ethnic group consciousness sustained over a long time and based on a sense of distinctiveness, a common history and the believe in a common fate"; "the possibility of a distinctive yet creative and enriching life in host countries with a tolerance for pluralism". Work migration is an important characteristic for describing social networks and the structure of ethnic organizations created by Azeris in emigration. But there also does not happen to be some united group consciousness in the case of the phenomenon of "Azeri diaspora" being constructed.

Another famous definition belongs to Sheffer (2003: 9-10): “An ethno-national diaspora is a social-political formation, created as a result of either voluntary or forced migration, whose members regard themselves as of the same ethno-national origin and who permanently reside as minorities in one or several host countries. Members of such entities maintain regular or occasional contacts with what they regard as their homelands and with individuals and groups of the same background residing in other host countries. Based on aggregate decisions to settle permanently in host countries, but to maintain a common identity, diasporas identify as such, showing solidarity with their group and their entire nation, and they organize and are active in the cultural, social, economic, and political spheres".

Diaspora is also considered as a real, joint group, which once formed and then remains practically unchanged. These definitions do not aim to try to see the phenomenon of diaspora as a process. A process in the course of which there can be ups and downs in the political and/or any other activity. The republic gained independence in the course of the Armenian-Azerbaijani conflict over Karabakh (1988-94) which, as it quite often happens, caused a mobilization of ethnic Azeris who were living in Germany, Russia or USA at the time and sought to give support to their political homeland (Demmers, 2005). As early as the early 1900s, the country of origin tried with a varying degree of intensity to use this activity which spontaneously emerged and aimed to support the political motherland. At the same time, the authorities of their political homeland also made efforts to mobilize ethnic Azeris around the world, confirming in this way the thesis that "The formation of 
diaspora is therefore an issue of social mobilization" (Sökefeld, 2006: 268). Time happens when the idea to establish trans-state business networks between the host country and the country of origin acquires special popularity, like it has been happening in recent years. However, these splashes of interest are normally temporary and often fade out in the second or third generation of emigrants' descendants. Cohen (1996: 515) himself too stresses that the criteria he singles out should be viewed as "one of 'common features', to indicate that no diaspora will manifest all features".

The main problem of all definitions cited is that they originate from the idea of the existence of various diasporas as real communities whose members are consolidated and invariably active in their relations with homeland. Or, as Tishkov (2003: 440) notes, "the main weakness in the interpretation of the historical phenomenon of diaspora in modern literature is the essentialist reification of diaspora as collective bodies ('stable aggregates'), what is more, not only as statistical multitudes but also as culturally homogenous groups, which with a more sensitive analysis is almost impossible to establish". And even if some of the criteria suggested by Cohen, Safran or Sheffer can be applied when describing social networks and structure of ethnic organizations created by Azeris in emigration, all definitions proposed do not in any way explain the diaspora policy of the Azerbaijani political regime. It is this policy of the regime that runs the country which is described as the political motherland for all Azeris or, to be exact, as the specifics of the mechanisms of its implementation, which to a considerable extent identifies the specifics of the phenomenon which the authorities describe as "Azeri diaspora".

In this connection, I see as the most constructive the approach proposed by Brubaker (2005: 13) who notes that "rather than speak of 'a diaspora' or 'the diaspora' as an entity, a bounded group, an ethnodemographic or ethnocultural fact, it may be more fruitful, and certainly more precise, to speak of diasporic stances, projects, claims, idioms, practices, and so on". Based on this position, we can analyze not only mechanisms of implementation of also the "styles" of the political project of the Azerbaijani authorities and the activities of ethnic activists in emigration which are aimed at constructing an ethno-national "Azeri diaspora". These "styles", largely identified, as the background of those who created and are implementing this project, give special specific features to the networks of emigrants and the organizational structure that seek to unite all Azeris in host countries into some sort of a united community.

It is excessive to develop a discussion about the inflation of the instrumental meaning of the term "diaspora" itself which has been more frequently used in relation to numerous emigrants' social networks and communities that appeared in the $20^{\text {th }}$ century, for very different reasons, in different countries of the world. However, we share the position of Brubaker (2005: 3) who stresses: "If everyone is Diasporic, then no one is distinctively so. The term loses its discriminating power - its ability to pick out phenomena, to make distinctions. The universalization of diaspora, paradoxically, means the disappearance of diaspora".

Therefore, hereinafter the mention of the terms "diaspora" and "diaspora politics" in the text will only reflect the official discourse, which is constructed by the political regime in Azerbaijan and within the space of which in host countries Azeris are repre- 
sented as a homogenous and joint ethno-national community, "diaspora". Since the early 1990s this term has been widely and habitually used in Azerbaijan (like, actually, by ethnic activists in emigration too) by officials, all possible sorts of experts (political analysts, sociologists, historians, etc.), journalists and regular people.

The authorities in the political homeland (Azerbaijani Republic, AR) do not put in the notion "Azeri diaspora" the common characteristics of similar communities as having "catastrophic origin and uncomfortable outcome" (Cohen, 1996: 515). The main criterion is the way the authorities in the homeland describe ethnic Azeris who have for various reasons ended up outside their official nation state. Azerbaijan "becomes an external national "homeland" where "political elites construe certain residents and citizens of other states as co-nationals, as fellow members of a single transborder nation, and they assert that this shared nationhood makes the state responsible, in some sense, not only for its own citizens but also for ethnic co-nationals who live in other states and possess other citizenships" (Brubaker, 1996: 5). Such an attitude from the authorities covers all ethnic Azeris who either permanently or temporarily (including even students) reside abroad.

\section{CRITERIA FOR ASCRIBING ONE TO "DIASPORA"}

The criteria that can be used to identify who should be described as Azeri are given in Article 1 of the current version of the "Charter of Solidarity of Azeris of the World" headlined "The Ethno-Social and Philosophical Notion 'Azeri", in which "Azeris are united by such ethnographic-cultural features as language, religion and spirituality". The structure of the notion of Azeri includes: first, Azeris themselves (here the authors apparently mean ethnic Azeris or, as it became commonplace to think in the post-soviet period, Azeri Turks), second, "Ethnic minorities who live in Azerbaijan and have merged with Azeris of Turkic origin"; and finally, in the spirit of modern liberalism, anyone that regards themselves as Azeri.

On the whole, the approach to the definition of "nation" is essentialist in nature. At the same time, the Charter reproduces, to a considerable extent, ideas that have already been laid out in the law "On state policy towards Azeris living abroad". This law was endorsed by Heydar Aliyev on 27 December 2002, a year after the holding of the First Congress of Azeris of the World (November 2001). Following the spirit of the law, the authors of the Charter point out that former (or those who have not lost that status) citizens of the Azerbaijani SSR or independent Azerbaijan who "think of themselves as Azeris in terms of their ethnic, linguistic and/or historical ties" are considered to be Azeris living abroad.

Authors of the Charter write that "the fact that many Azeris are citizens of various states must not prevent their ethnic unification". The first (and the most important) reason why dispersion should not be an obstacle for unity is the existence of the independent Azerbaijani Republic. Other criteria are "Historical motherland etched in the ethnic memory of the people", then follow traditions, language, religion, ethno-social outlook, ethno-cultural system of rules, ideology of "Azerbaijanizm" (Azəbaycançılıq i.e. Azerbaijani nationalism), and finally the fact that there is a "national leader" in the shape 
of Heydar Aliyev who declares: "I have always been proud of being an Azerbaijani!" that are described as some ideal of attitude to the imaginary community of Azeris. The charter calls for following such a manifestation of particular significance of ethnicity. "Dear compatriot! Be proud of the fact that you're a child, descendant of this ancient land, a representative of a nation with a glorious history! Be proud of being an Azerbaijani!".

During the development of the text, recommendations, feedback and proposals from various organizations, including diaspora ones, were used as a basis. This is not only about ethnic organizations of Azeris, as the Society of Azeris of Latvia, the Council of Azeris of America, the Society of Azeris of Mainz, the Azerbaijani House of Belgium... Headlines were also received from the Turkish diaspora organization Community of Turks of Berlin. However, in this case too the political regime exercised total control over the procedure of the putting together of the charter: headlines came in from the department of public and political issues under the presidential Administration, the country's Foreign Ministry, the State Committee for Work with Religious Bodies, and, finally, the Research Institute for Philosophy and Political and Legal Studies of the Azerbaijani Academy of Sciences.

The working group that put together the text of the Charter includes only specialists in humanities (a total of 19 people) that work in various universities of the country and research institutes of the Academy of Sciences. Based on criteria of singling out Azeris and modern ideology of Azerbaijani nationalism, specialists also provide a definition for "diaspora" which "a united and inseparable part of the Azerbaijani people that lives outside its historical Motherland". In this case, this is about quite a common definition that implies, as Tishkov put it, a must-presence of such a "conditional category" as motherland.

\section{IMAGINARY STATISTICS}

At the same time, the description of "historical motherland" of all Azeris contains also a statistical discourse in the context of which Azeris become a large ethnic community whose members live in 70 countries. At the same time, belief in the presence of a "historical motherland" for everyone and imaginary statistics of the number of residents in host countries become important elements of constructing the image of a large and consolidated "Azeri diaspora". In the context of the diaspora discourse it is common to believe that it is the considerable number of residents said to be a part of the ethno-national diaspora that largely determines the level of the influence of the community in one or another country. The constructed official statistics of the number of ethnic Azeris looks somewhat contradictory. On the official (governmental) site dedicated to the $1^{\text {st }}$ Congress of Azeris of the World, which was held in Baku on November 2001, general information has been posted dedicated to the "Azeri diaspora"2. In addition to everything else, it says that "According to rough estimates, about 45 million Azeris live in 70 countries of the world, including Azerbaijan, today".

$2 \mathrm{http://www.diaspora.az/qurultay/d-ru.htm}$ 
By the time the $2^{\text {nd }}$ Congress of Azeris of the World was to be held (March 2006) the State Committee for Work with Azeris Abroad prepared a documentary with the eloquent title "We are a 50 millions strong people". Ever since, this film, not the only of this kind, has been one of the mandatorily viewed ones at various official events linked to the implementation of the diaspora project. The important ideological significance of such films for the ruling regime in the country becomes clear if one remembers that the opening of this $2^{\text {nd }}$ Congress started with the demonstration of another one of them, entitled "We, Azeris". This film aims to propagate within the "diaspora" the activities of the former president (and the father of the incumbent), Heydar Aliyev.

President Ilham Aliyev's speech actually started with the mention that "Today a wonderful film has been screened here. We once again recalled the beautiful moments when at the initiative of the national leader of our people, Heydar Aliyev, five years ago took place the 1st Congress of Azeris of the World which played an exceptional role in the modern history of Azerbaijan" . A series of such films was prepared specially to present the ideology of the official diaspora project to Azeris in emigration. Although these films are not some kind of secret information, in Azerbaijan itself they are practically inaccessible for viewing. That is to say, this propagandist material is not aimed for domestic audiences.

Considering the special significance that is attached to statistics, it is natural that the compilers of the Charter dwelled upon the issue of the number of ethnic Azeris. This text says there are as many as over $50 \mathrm{~m}$ Azeris. According to "rough estimates" by the compilers, four out of five Azeris live in their "historical motherland" and only one out of five outside it.

All the official ethnic Azeris that live outside their "historical motherland" in the context of diaspora discourse become a united and homogenous ethno-national diaspora, regardless of whether they previously were (or currently are) citizens of the Azerbaijani Republic itself, Georgia, Iran, or Turkey. The mention of these countries is no coincidence: outside the Azerbaijani Republic itself, Azeris populate precisely these three countries and it is this populated territory that is described as "historical motherland" in the context of Azerbaijani nationalism. Therefore, considering the specifics of the political areal populated by ethnic Azeris, the same Charter pays great attention precisely to these issues. At the same time, although explicitly Iran, Georgia and Turkey are not mentioned, it is these territories populated by ethnic Azeris that the authors of the Charter refer to.

Outside the "historical motherland", special importance is attached to "Azeri diasporas" in those countries which, in the opinion of the authorities in Azerbaijan, play a leading role in the world political arena. In the CIS that is the Russian Federation where the largest number of Azeris live who for various reasons ended up in this country. Among the EU countries, special significance is attached to Germany where currently the largest number of Azeris who migrated to Europe lives. In addition, in this case, special hopes are pinned on the establishment of close contact with Turkey too. Finally, special significance

3 Azerbaijani president's speech at the $2^{\text {nd }}$ Congress of Azeris of the World, in Bakinskiy Rabochiy, $\mathrm{n}^{\circ} 48,03.17 .2006$. 
is attached to the activities of the "diaspora" in the USA as the only super power of the modern world. Besides, the increasingly more active embassies of Azerbaijan in Russia, Germany and USA, also very active Consul-general in Los Angeles, are acting as coordination centers providing for cooperation between diaspora organizations in these countries and the authorities of the political motherland.

Unification of all these Azeris who have for a variety of reasons and at different times found themselves outside their country of origin (i.e. Azerbaijan itself, Iran, Turkey or Georgia), becomes possible only within the context of diaspora discourse and the practice of bureaucratization of emigrants' social networks.

\section{THE DISCOURSE AND BUREAUCRATIC PRACTICES OF CONSTRUCTION OF DIASPORA}

The mechanisms of the implementation of the political project for the construction of diaspora come down to two major and inter-related practices.

\section{The First is Discourse Practice}

"Via the discourse practice, some kind of construction of diaspora as a group occurs" (Tishkov, 2003: 180). Via discourse practice, all those whom ethnic activists and officials in the political motherland identify as ethnic Azeris are viewed as "Azeri diaspora", quite often using statistics as a habitual "institution of power" (Anderson, 1998: 163). At that, the extent of the intensiveness of daily ties and contacts between actors, who are ascriptively viewed as a diaspora community of Azeris, no solidarity of their political or any other interests is discussed. In the context of, as Billig (1995: 102) put it, "the rhetorical clichés of political discourse" almost all Azeris in all situations are a homogenous and united community (ethno-national diaspora) that is invariably loyal to the construct of ideal political motherland-nation.

Yes, on the one hand, said the previous Azerbaijani President Heydar Aliyev in his speech at the $1^{\text {st }}$ Congress of Azeris of the World ${ }^{4}$, "We believe that every one of the Azeris living in various countries have settled their life in that country in the way they want. Every one of them is a citizen of that country, and must live by its laws and rules and go along the path selected". However, this loyalty to the host country "must" (this imperative tone is also present in the text of the Charter) be accompanied also by loyalty towards the political motherland, and "Azeris living outside Azerbaijan must today maintain closer ties with independent Azerbaijan".

However, preliminary observations make it possible to assume that the daily routine of many statistical Azeris living in Russia, Germany or USA, is to a much larger extent determined by how they ended up in one of this countries or from what country they emigrated, and not by the intensiveness of contacts with other representatives of the

$4 \mathrm{http}: / /$ www.diaspora.az/qurultay/speech-ru.htm 
imaginary community or ties with their official homeland and intensive activities in the cultural and other spheres.

The routine, practically daily reminder in the media about the activities of the "Azeri diaspora", constant interviews with ethnic activists, officials and various sorts of public figures talking about their successes in the implementation of the project for the construction of diaspora, form the discursive effect of truth within the context of which "diaspora" acquires the features of a true, large, joint and homogenous community united by common goals. Effectively, "With the help of the word 'diaspora', 'communities', etc., a desirable image of reality, which is presented as this very reality, is created" (Kosmarskaya, 2002: 114). At the same time, the very realism of such social group as nation, the giving to it of essentialist characteristics, and also the idea of inevitable solidarity of all actors ascribed to it do not contradict the simple "common sense" of those carriers of power and intellectuals who implement the diaspora project. All of them (both presidents, the head of the State Committee for Work with the Diaspora, most officials of various ranks, intellectuals working on the text of the Charter, etc) socialized in the Soviet times, when institutionalized ethnicity was a major basic characteristic of any USSR citizen. In accordance with Brubaker (1994), Malakhov (2007: 50) notes, "the ascribed 'ethnicity' (i.e. identified by the authorities and not by the self-consciousness of individuals) was interiorized by people and gradually turned from an external identifier into part of (self)-identity. Hence originated such peculiarity of political thinking as methodological ethnocentrism - view of society as a conglomerate of 'ethnoses' ('peoples'). This type of thinking is today shared both by mass consciousness and a considerable part of intellectual and political elites".

\section{The Second is the Bureaucratic Practice of the Production of a Hierarchically Structured and Co-subordinated Organization Structure}

A rapid bureaucratization of social networks of Azeris in the world occurs, as a result of which an organizational structure of diaspora is constructed. Here I mean a process where increasingly more new diaspora organizational structures are produced "including transnational ethnic and hometown associations" (Henry, et al., 2004: 841) within the context of actualization of contacts with political homeland. With an ever increasing intensiveness, during almost all post-Soviet years ethnic activists have been making attempts, as Benedict Anderson put it, with the support of the state machine of the country of origin, to construct an ethno-national Azeri diaspora in Russia, EU countries, USA or any other states as collective subjectivity (Anderson, 1998). As a result, more and more often ethnic Azeris, who temporarily or constantly live in CIS and EU countries, USA or Canada and so on are referred to as a homogenous group (Brubaker, 2002: 163-167) - the "Azeri diaspora".

Within this context diaspora politics contains certain contradictions. On the one hand, the policy of unification with the Turkish diaspora is declared, which, in the opinion of Safran (1991: 91), can, with a certain degree of proximity, be described as an ideal type of diaspora. It is this element of the diaspora politics that can be especially topical for the community of Azeris in Germany and USA, but not in Russia. On the other hand, 
borders between ethnic Azeris from Turkey (so-called Turkish Azeris) and actual Turks are constructed.

At the same time, despite the active attempts by the Azerbaijani authorities to actualize the ethnic identity of emigrants and step up their contacts with their "official motherland", "Identity, in principle, is a runny and mobile substance, therefore the space of choice for members of the diaspora here is sufficiently big" (Kolsto, 2001: 7). Eventually, despite the active diaspora policy of the Azerbaijani regime, the entire activity immediately in emigration comes down to the activity of a number of ethnic activists who for a variety of reasons participate in the work of organizations that they themselves created. In the meantime, the activity of the relatively few ethnic activists and organizations most often does not have practically any influence on daily practices ascribed to "diaspora", according to Brubaker (2005: 13), "the majority who do not adopt a diasporic stance and are not committed to the Diasporic project". We should stress that contacts with ethnic organizations, and, moreover, immediate participation in their work is topical, to very varying degrees, only for a handful of emigrants.

However, such a low activity in emigration is not an obstacle for the increasingly numerous ethnic organizations to become a major element of the discourse within the space of which constructs of the ethno-national "Diaspora" of Azeris acquire the features of a real consolidated community. The demonstration of the process of continuous production of ethnic organizations that almost invariably become partners for the authorities, as a system-forming element of constructing an ethno-national diaspora, is present in speeches, statements and interviews of politicians. As a result of the implementation of the Diaspora-Building project by mid-2007, in the words of the "Minister of Diaspora of Azerbaijan Mr. Ibrahimov"5, "more than 300 Azeri communities are operating in most of the countries of the world, and the process of establishment of diaspora organizations is continuing presently too" 6 .

The logic of multiplication of the quantity of organizations as a symptom of success of diaspora-building can be understood primarily proceeding from the premises that there had almost not been these kinds of structures by the moment Azerbaijan gained independence in $1991^{7}$. Their current ever-increasing number is becoming a symbol of the strengthening of the positions of independent Azerbaijan in the world. At that, certainly, one should not put the growth of the number of officially registered ethnic organizations down only to the efforts of the authorities in the official homeland. The spread of the disposition for building these kinds of structures was caused by the very emergence of an independent political homeland, without any deliberate policy on the part of the

5 A female informant, about 45, in Berlin gave this apt definition to the status of Nazim Ibrahimov, head of the State Committee on Relations with Azeris Living Abroad.

6 Fifth anniversary of the establishment of the State Committee on Relations with Azeris Living Abroad. Available at: http://www.azinba.az/news_detail.php?news_id=5397

7 However, small organizations did exist before. For example, 2008 marked the $50^{\text {th }}$ anniversary of the above-mentioned "Community of Azeris of America", which is led by Tomris Azeri. But it would be an absolute exaggeration to talk about any serious movement or policy of construction of ethno-national diaspora back in the Soviet period. 
authorities, while some revival in their activities coincided with the transformation of the Karabakh conflict into a full-scale war between Azerbaijan and Armenia.

These and many other ethnic organizations carry out the function of presenting the ever-growing organization and activities of ethnic "Diaspora" within the discourse. However, according to my observations, in the emigration the numerous numbers of ethnic organizations, on the contrary, have to do with the absence of a real unity of goals and interests among the residents. At the same time, a fast growth of their number shows the specificity of the policy of influence on emigrants, on the part of the authorities of the official homeland. The bureaucratic system of the authorities consolidated within the space of the political homeland can build its relations only with a certain structure (envisaging a certain hierarchy) consisting of ethnic organizations registered in one or another host country. The realization of contacts, establishment, as it is declared, of dialogue and interaction between the authorities and the "Diaspora" becomes possible only through relations with official organizations which assume the task of presenting all Azeris abroad. These organizations increasingly more often arise in response to the expectations of the authorities of the official homeland, while ethnic activists creating them in host countries, increasingly more often seek to implement transnational economic projects through these contacts. The authorities, certainly, seek to choose partners, and through the discourse practice officially endorses their status, confirming their right to represent the entire community in one or another part of the world. This response (from the political homeland and ethnic activists in emigration) aspiration to produce a sort of a strictly hierarchised structure of the "Diaspora" is based on the fact that common political, cultural and economic interests are shared, to this or other extent. Starting with organizations that apparently represent communities of cities (for instance, "Mainz-Azerbaijan"), and then communities of one or another EU country (for instance, "the Advisory Council of Azeri diaspora organizations", operating in Germany, was founded in Mainz in July 2007). These organizations are increasingly more often divided into youth ones and not. All these structures operate with support from and/or under monitoring by the Azerbaijani Embassy in Germany. Then follows a body apparently uniting all Azeris of Europe (for instance, the Congress of European Azeris) and above them is an organization that apparently represents all Azeris of the world and coordinates their activities - "Coordinating Council of Azeris of the World".

This structure, which is uniform from local organizations up to those that represent all ethnic communities, and which is constructed by the principle of state vertical of power, seems to be simple, clear, transparent and convenient to control. At the head of this structure there is "a minister for the Diaspora", already subordinated, directly, to the pan-Azeri President. The latter describes the present stage of the implementation of the project as follows: "We are now finishing the structuring of all Diaspora organizations of Azeris living abroad. We have held the World Congress of Azeris two times. At least 50m Azeris live in the world. Certainly, the strong Azerbaijani state should provide support to all our compatriots. At the same time, strong Diaspora organizations help assert our national interest. Until recently, some five to six years ago, these organizations operated practically on a voluntary basis. In this connection, by the decision of our national leader Heydar Aliyev, the State Committee for Work with Azeris Living Abroad was created. It deals with problems facing the Diaspora only. At present, these organizations are struc- 
tured in every country. We have recently opened more than 30 embassies in different countries, and now their number has increase to more than 50 in the world. All embassies and Diaspora organizations in those or other countries operate together. It turns out that this is a two-way street: we render them assistance, and they, in turn, help their country".

Thus, within the space of the diaspora discourse and the related practice of bureaucratization of emigrants' social networks, Azeris in various countries of the world become a true united group - "diaspora". Within the space of the diaspora discourse, emigrants who have for a variety of reasons found themselves in different countries of the world, including Azeris, citizens of different countries unite and acquire features of a homogenous joint group. In the meantime, description, in the context of the diaspora discourse, of the image of the joint and large diaspora penetrated and controlled by the united and hierarchically co-subordinated bureaucratic structure of ethnic organizations is a visible confirmation of the real existence of a large diaspora community of Azeris.

\section{THE GOALS OF THE DIASPORA POLITICS}

The goals of the diaspora politics declared by authorities and diaspora activists that actively participate in its implementation can apparently be very different. This is for example an intensification of the participation of diaspora representatives in an as wide promotion as possible of official Azerbaijani position in the Karabakh conflict. In this context, "Diaspora politics may be more a result of conflict than its cause" (King and Melvin, 1999-2000: 137). Resolving the Karabakh conflict assumes, for example, a certain "struggle with world Armenians", "which", in the opinion of President Ilham Aliyev, "We [i.e. Azeris of all the world] should oppose with unity and force". He said these words in his speech on the occasion of the opening of "The XI Congress of Friendship and Brotherhood of Turkic States and Communities", which was held in Baku in November 2007. The constant mention of the Armenian Diaspora demonstrates the fact that the official project for the construction of "Azeri diaspora" is very largely built on stereotypes about the Armenian Diaspora.

In addition, the current political regime seeks to use ethnic (diaspora) organizations and networks to spread the official national ideology of "Azebaijanizm", adopted in the Azerbaijani Republic, among emigrants. This is about the post-Soviet version of the "ethnogeny" of Azeri Turks, history of statehood, and the image of "historical enemies" that occupy an important place in the post-Soviet version of history. Ethnic activists and organizations are, apparently, more intensively joining this activity, providing opportunities for emissaries from their political homeland to deliver lectures, spread literature (of particular importance here are history textbooks, including those developed for secondary schools), to hold various exhibitions or collective events.

The tangle of opinions, interests and expectations of intellectuals, all sorts of experts and politicians concerning Azeris abroad, is also shaped depending on the foreign

8 Exclusive interview of Azerbaijani President Ilham Aliyev to Kazinform's correspondent in Baku. Available at: http://www.day.az/news/politics/88011.html 
policy preferences of the authorities and politicians in official homeland. Thus, an active involvement of the "Azeri diaspora" is presumed in the campaign for the recognition of injustice in the assessment of the events of 1915 in Anatolia, namely of the genocide claims put forward to Turkey, present-day major ally of Azerbaijan. The declared foreign policy priorities acquire a special meaning too (for example, orientation towards participation in European and Euro-Atlantic structures). It is within these contexts that an increasing popularity is gained annually by the ideas that present Azeris in Germany, the USA, Russia, or in any other country that has weight in world politics, as a certain significant political resource which the authority in the country of origin can and should use to achieve certain political preferences. On the whole, at the level of declarations, this primarily means advertising "democratic transformations" carried out by the ruling regime in Azerbaijan, presenting the "young" Azerbaijani community abroad.

Special place in the diaspora policy is given to the holding of collective events on the occasion of various memorable dates. These events are described in the context of the diaspora discourse as facts that confirm the invariable unity of the large community of Azeris of the world. Collective events in the "diaspora" that are of interest to the Azerbaijani regime, are also held, in addition to marking events of the Armenian-Azerbaijani confrontation, on the occasion of symbolic dates of the establishment of independent Azerbaijan, and are also connected with the propagation of the activities of the former president (and the father of the incumbent), Heydar Aliyev.

The conflict over control of the Karabakh region (1988-94) resulted to the Azerbaijani-Armenian confrontation becoming retrospectively translated onto many events that had occurred much longer before it. These include the events of 1918 in Baku, when pogroms took place in Muslim neighbourhoods in the city as a result of a political confrontation between Bolsheviks, who had attracted to their side troops controlled by Armenian nationalists (Dashnaks), and Musavatists (Turkic nationalists). As a result, about 10,000 were killed. This event has been referred to in the post-soviet period. After Heydar Aliyev's decree of 1996, the events of March 1918 started to be interpreted as genocide. Currently the authorities call on ethnic activists to hold collective events on 31 March. The idea of this genocide of Azeris also becomes some kind of a countertheory against the Armenian genocide in Anatolia in 1915-18. The Azerbaijani authorities actively lobby the idea of the need to back the Turkish authorities and Turkish diaspora organizations that deny the genocide. Among other events, the events of 20 January 1990, when, according to official reports, up to 132 people were killed when soviet reports were deployed to Baku which the USSR authorities were practically not in control of, have acquired the greatest significance; and ethnic cleansing in the town of Xocali (events of the Karabakh war). I should say that far fewer emigrants participate in these events than in events held on the occasion of holidays.

The holidays that have been established in the post-soviet period and are backed by the Azerbaijani authorities can be said to include the Day of the Solidarity of Azeris of the World (31 December), Day of the Republic, which is considered to be the day of the revival of Azerbaijani statehood (28 May), and, to a lesser degree, Independence Day (18 October). 
Finally, collective events (concerts, conferences, rallies, etc.) linked with events of the policy to commemorate the activities of the previous president gain an everincreasing significance. After his death in 2003, he, largely similarly to Atatürk, becomes in the context of the official discourse the symbolic "national leader" (ideal politician and Azerbaijani) for the entire nation. Therefore, not only anniversaries but even simply the days he was born and passed away, and dates linked to his rule ("Day of Salvation of the Nation", etc.) are hailed to be marked within the diaspora.

The implementation of all these goals, in the authorities' opinion, implies creation of an Azeri political lobby in the host countries, primarily, in the modern world's leading political and economic centres (USA, EU, and in a way Russia). Therefore, the policy of construction of "Azeri diaspora" actually aims to create own no less united and joint ethnic community -Diasporic "Lobby" which would be able to oppose the considerable Armenian Lobby with a no less serious political power. The aim of such policy of ethnic activists in emigration may be described by a phrase by the ex-president of the Congress of European Azeris (CEA) Bahaddin Kaya: "Now, in my opinion, the main task of the Diasporic movement must be to create a powerful propagandistic Turkic Lobby abroad".

And here, special interest arises in cooperation with the Jewish diaspora. Ethnic Jews, natives of Azerbaijan, are perceived by the authorities as a very important resource for creating a political lobby. In the project of construction of the ethno-national "Azeri diaspora", this disposition for cooperation with the Jewish Diaspora (and not only Turkish diaspora) is being increasingly often proclaimed by the Azerbaijani authorities too. Thus, for instance, Nazim Ibrahimov, said in July 2007: "The State Committee has long been seeking a key to creating an Azeri diaspora organization in US states like California, where traditionally the Armenian lobby is powerful. And we've found such a key. The Jewish diaspora, operating here, is ready to help us organize our diaspora"10.

Here are views on cooperation from a European perspective by Natig Ahamirov, the President of the Congress of European Azeris (CEA): "Azeri communities closely cooperate with Jewish diaspora organizations. The CEA is trying to improve its work in this sphere. We consider that we can use the potential of Jewish communities in those countries where Azeri communities under-operate and have limited opportunities to influence social and political processes. The centuries-old friendship between our peoples gives us wide opportunities for this cooperation. We just need to efficiently take advantage of this potential. Recently, the Azerbaijan-Israel international Association was established in Israel. The CEA is planning to establish close relations with this structure. In one word, we are thinking about joining our efforts in all spheres"11.

The active participation of ethnic Jews in the work of the Azerbaijan community (consisting of, in a wide sense, all natives of Azerbaijan) is not something out of the ordinary and has to do with a circumstance which Safran (2003: 390) well describes as "the persistence of fraternal societies based on common local background" as Niznir

9 See: http://www.azerizv.az/article.php?id=5698

10 See: http://www.day.az/news/politics/85680.html

11 See: $\mathrm{http}: / /$ news.trend.az/index.shtml?show=news\&newsid=953305\&lang=RU 
(2003) does too. This background helps preserve the Jewish "subcommunities" in Israel, determined by their country of origin - "Anglo-Saxons", "Easterners", "Russian" and others. In the case of ethnic Jews, natives of Azerbaijan, these are Mountain Jews from Quba town and Baku city, and Ashkenazi Jews from Baku.

\section{MAIN EVENTS OF DIASPORA PROJECT}

As already indicated above, it was Heydar Aliyev who implemented the project for constructing an "Azeri diaspora". In the post-soviet period, he was Azerbaijan's president from 1993 to $2003^{12}$. The many years of being in power made him, as Bourdieu (2007: 84) put it, "a trusted person who had the power to "form a group". It was his activities, back in the years when the USSR existed, that largely led to the establishment in Russia of an intellectual layer from among ethnic Azeris, who became ethnic activists when the USSR collapsed. Much later on, Heydar Aliyev said in his speeches that his had been conscious of his actions. However, he never claimed that he could foresee the collapse of the USSR and that Azeris would turn into "diaspora".

Thus, speaking at the Founding Assembly of the "All-Russian Azeri Congress" (AAC) on 22 June 2000, he stated that: "previously, always, when I worked in Azerbaijan, I said, and this was my firm belief both then and today, that no people can develop at the pace of the entire world community, the entire world system if it isolate itself in the space boundaries of its territory. I.e. I have always opposed the feeling of national restriction"13. Such a clear-cut position was, probably, conditioned by his personal experience. Heydar Aliyev himself studied for some time in Leningrad, and spent a long time living and working as one of the USSR leaders in Moscow and knew well that in a host of specialities a high level of education as a major factor of mobility and demand in the modern world is often possible only if one receives education outside Azerbaijan. Education in a number of specialities in the Azerbaijani SSR was not accessible in principle. "Guided by these principles, when I worked as Azerbaijan leader in the 1970s, and up until the end of 1982, then I sometimes even administratively made sure that our young people, youth, went to other republics of the Soviet Union to receive education. Back then, we did not have opportunities other than the Soviet Union, i.e. within the Soviet Union. Azerbaijan was the only republic which managed to achieve major privileges in this regard. From 1970 on, I set a task to send many Azeris outside Azerbaijan, primarily to Russia, primarily to Moscow, Leningrad, and other major centres for higher education, centres for science, centres for culture" (Bakinskiy Rabochiy, 28 June, 2000).

In addition, Heydar Aliyev himself, due to circumstances, took part in the first collective event of Azeris in Russia in 1990. That was "January 1990" - a protest against the deployment of Soviet troops to Baku on the night of 19-20 January 1990. According

12 The current president also points to this: "the idea of organizing and the initiative for consolidation of Azeris of the world were put forward by the great leader Heydar Aliyev", in Bakinskiy Rabochiy, 28 June 2000.

13 Azerbaijani President Heydar Aliyev's speech at the founding assembly of the all-Russian congress of Azeris in Moscow, in Bakinskiy Rabochiy, 28 June 2000. 
to my observations, by the end of the 1980 s, although no-one was yet expecting the disintegration of the Soviet Union, which was coming in the not distant future, Azeris (often under the influence of policy conducted by Russian officials who sponsored the production of ethnic organizations) started gradually recognising themselves as sort of a community that had found itself outside their country of origin. The January 1990 events in Baku, when 132 people were killed and many injured, actualized the feeling of being in emigration. Heydar Aliyev was the central figure among those publicly expressed their protest against the actions of the soviet leadership. Possibly, precisely these events reaffirmed his belief that the networks of ethnic Azeris not only in Russia might become a significant resource that could be mobilized to support their country of origin.

The first important step of the then planned diaspora policy was a decree by $\mathrm{H}$. Aliyev declaring 31 December to be the Day of Solidarity of Azeris of the World. It is remarkable that this decree was adopted even before 16 December 1991, i.e. when he held the post of chairman of the Supreme Assembly of the Naxcivan Autonomous Republic ${ }^{14}$. Only a charismatic leader like Aliyev could afford to take this step, which, undoubtedly, went far beyond a regulation by a regional leader: a powerful symbolic capital, which he was the only one of all Azerbaijani politicians of the time to have allowed him to perform this gesture. In essence, that was a call addressed to all Azeris living outside the Azerbaijani Republic to remember their "political motherland". This was although the first but already a very important symbolic act demonstrating not only the future change to the status of Azerbaijan in the world but also a change of the very rules of the political game which would now be played not in a space confined by an "iron curtain" but on a wide global scale.

The very prospect for conducting a purposeful policy in relation to the diaspora became a reality once Heydar Aliyev became president. Every year, since December 1993, the president's address to the Nation has turned into traditional address to the global community of Azeris living in a variety of places around the world. It was Heydar Aliyev who made the annual global address a habitual thing that actualises the idea that all ethnic Azeris belong to a united and joint 50 millions-strong ethnic community ${ }^{15}$. "On this remarkable day, I congratulate all Azeris of the world on the Day of Solidarity, and wish everyone successes in the name of an even brighter future for Azerbaijan and unity and solidarity of our people". ${ }^{16}$

However, main events of the diaspora policy took place in the 21 st century. As Aliyev put it, an important stage for the unification of Azeris took place on November 2001 - the $1^{\text {st }}$ Congress of Azeris of the World, timed to coincide with the $10^{\text {th }}$ anniversary of the appearance of independent Azerbaijan on the world map. His successor said that "it was after this congress that the State Committee for Work with Azeris Living in Foreign Countries was set up in Azerbaijan under Heydar Aliyev's decision. This committee carries out quite successful work to organize Azeri diaspora. The law on state policy

14 Regulation by the Supreme Assembly of the Naxcivan Autonomous Republic "On the day of solidarity and unity of Azeris of the world", dated 16 December 1991.

15 In fact, the very concept of united 50m-strong community was formulated at a far later stage.

16 See Bakinskiy Rabochiy, 01.04.1994 (newspaper in Baku, in Russian). 
connected with Azeris living abroad was adopted in 2002, which laid the legal basis of this movement. All these measures and all these steps are yielding fruit. Our compatriots in the world are getting organized and playing an increasingly more active role in the life of the countries they live in. certainly, on the one hand, this strengthens their position, and on the other, this strengthens the positions of the independent Azerbaijani Republic and multiplies its authority" ${ }^{17}$ As a logical continuation of the diaspora policy (at the $1^{\text {st }}$ congress it was decided to hold such events every five years), the $2^{\text {nd }}$ Congress took place on 2006. This congress renewed the members of the "Coordinating Council of Azeris of the World" which was created at the $1^{\text {st }}$ Congress. This Council comprises 109 people, 45 of whom permanently live abroad. The number one member of the Council is the current president, Ilham Aliyev.

The latest significant event is one of the first decrees that Azerbaijani President Ilham Aliyev signed after his re-election to the post in October 2008. This is the decree "On the establishment of the State Committee of the Azerbaijani Republic for Work with Diaspora", which was signed on 11.19.2008. Naturally, everything was confined to the mere renaming of the already existing "Commute for work with Azeris living abroad". However, the interesting thing is that the main declarative aim of this decree was to improve the structure of state management in Azerbaijan. In his speech at the $2^{\text {nd }}$ Congress of Azeris of the World, the current president re-affirmed his adherence to the diaspora policy which formed under his father. "Are we conducting our own foreign policy. And here, certainly, one can feel a great need for both your help and intensification in your overall activities. I have seen in the past few years that this work acquires coordinated forms - the work of the Foreign Ministry, the State Committee for Work with Azeris Living in Foreign Countries, Azeri diaspora bodies, events held in various countries, conferences, symposiums, other events connected with Azerbaijan - all this strengthens our positions and gives the world community true information about Azerbaijan"18.

\section{CONCLUSIONS}

It should be summarized that all attempts undertaken so far, as Anderson (1998: 44-45) put it, to construct, using the state apparatus, mathematics (statistics) and with the involvement of ethnic activists, an ethno-national Azeri diaspora as a collective subjectivity are still not producing much result. The disposition for a quantitative increase in the number of registered ethnic organizations acquires a key significance in the space of official discourse. In reality, basically quite a limited circle of ethnic activists mainly participate in their work. Having become partners of the authorities of their political homeland, they are given a status to represent the ethno-national community of a city, country, a part of the world... In this line of increasingly wider representations, the president of the political homeland now represents all "compatriots".

17 Ibid.

18 Azerbaijani president's speech at $2^{\text {nd }}$ Congress of Azeris of the world. Bakinskiy Rabochiy, $\mathrm{n}^{\circ} 48$, 03.17.2006. 
Implementing this project, the authorities prefer to cooperate with migrants that they view as having a status (ethnic activists, workers of science, culture and so forth). However, delegates of all possible congresses, forums and other collective events, represent, basically, themselves, and/or their personal interests or the interests of closest people to them (these are interests that are mainly connected to production of transnational business networks).

In reality, a significant part of Azeris abroad who do not want contacts with the embassy for different reasons (political refugees, illegal emigrants, etc.), is excluded from participation in the ethno-national community being constructed. Their presence in the official diaspora discourse is limited by statistics only. There is almost no intensive and successful interaction, and there will not be in the near future, among Iranian, Turkish and "Soviet" Azeris. The expanded cooperation of the ethnic organizations of Turks and "different" Azeris seems even less probable. On the contrary, ties with ethnic Jews, especially natives of Azerbaijan, are an important resource for arranging the activities of ethnic organizations.

Routine daily reminders in the media about a growth of successes of diasporabuilding acquire special significance for representing the ethno-national diaspora. The reality of "the Azerbaijan Diaspora" as a homogeneous and real group arises in the space of constantly increasing narratives both from the authorities of Azerbaijan (the president's appeals and statements, etc.), and from ethnic activists in emigration. This discourse construct is complemented by the imaginary statistics of the number of Azeris outside their political homeland as a millions-strong consolidated group acquiring features of being real against the background of a daily turn of news of events in the "diaspora".

So, behind the organizations there are only a handful of activists and their personal ambitions, behind statistics there are no real data, and the millions-strong consolidated ethno-national Azeri diaspora exists only as a discourse or, paraphrasing Irina Sandomirskaya, only when it is spoken about. At the same time, since 2001, when the symbolical $1{ }^{\text {st }}$ Congress of Azeris of the World took place, the political project of diasporabuilding has been acquiring features of a long-term arrangement and it cannot be ruled out that it may result in one or another degree of a successful building of a transnational community of Azeris or an ethno-political lobby in one or another country.

\section{References}

ANDERSON Benedict (1998) The Spectre of Comparisons: Nationalism, Southeast Asia and the World, London, Verso.

ANDERSON Benedict (1998) Imagined Communities: Reflections on the Origin and Spread of Nationalism, London, Verso.

BILLIG Michael (1995) Banal Nationalism, London, Sage.

BOURDIEU Pierre (2007) Sotciologiya sotcialnogo prostranstva, Moscow, Institute Experimentalnoi sotciologii.

BRUBAKER Rogers (1994) Nationhood and the national question in the Soviet Union and postSoviet Eurasia: An institutionalist account, Theory and Society, 23 (1), pp. 47-78. 
BRUBAKER Rogers (1996) Nationalism reframed: nationhood and the national question in the New Europe. Cambridge, Cambridge University Press.

BRUBAKER Rogers (2002) Ethnicity without groups, Archive Européenne de Sociologie, XLIII (2), pp. 163-89.

BRUBAKER Rogers (2005) The "Diaspora” Diaspora, Ethnic and Racial Studies, January, 28 (1), pp. 1-19.

COHEN Robin (1996) Diasporas and the nation-state: from victims to challengers, International Affairs, 72 (3), pp. 507-20.

DEMMERS Jolle (2005) Nationalism from without. Theorizing the role of diasporas in contemporary conflict, in T. Atabaki and S. Mehendale Eds., Central Asia and the Caucasus: Transnationalism and Diaspora, New York, Routledge, pp. 10-20.

FAIRCLOUGH Norman (1995) Critical Discourse Analysis, London, Longman.

HENRY Leroi, MOHAN Giles and YANACOPOULOS Helen (2004) Networks as Transnational Agents of Development, Third World Quarterly, 25 (5), pp. 839-855.

KING Charles and MELVIN Neil J. (1999-2000) Diaspora Politics: Ethnic Linkages, Foreign Policy, and Security in Eurasia, International Security, 24 (3), pp. 108-38.

KOLSTO P. (2001) Ykorenayushiesa Diaspori: Russkie v bivshix sovetskix respyblikax, Diaspori, 1, pp. 6-38.

KOSMARSKAYA N. (2002) Russkie Diaspori: Politicheskiye mifologii I realii massovogo soznaniya, Diaspori, 2, pp. 110-156.

MALAKHOV V. (2007) Ponayehali Tut... Ocherki o Natsionalizme, Rasizme i Kulturnom Pluralizme, Moscow, NLO.

MALAKHOV V. (2002) Preodolimo li etnotsentricheskoe mishleniye? in V. Voronkov, O. Karpenko and A. Osipov Eds., Rasizm v yazike sotsialnix nayk, Saint-Petersburg, pp. 9-22.

NIZNIK Marina (2003) The Russian Language as a Base Factor: The Formation of the Russian Community in Israel, in R. Münz and R. Ohliger Eds., Diasporas and Ethnic Migrants: Germany, Israel and Post-Soviet Successor States in Comparative Perspective, London, Portland, Frank Cass, pp. 355-369.

SAFRAN William (1991) Diasporas in Modern Societies: Myths of Homeland and Return, Diaspora, 1 (1), pp. 83-99.

SAFRAN William (2003) The End of "Normality": The Diasporization of Israel?, in R. Munz and R. Ohliger Eds., Diasporas and Ethnic Migrants: Germany, Israel and Post-Soviet Successor States in Comparative Perspective, London, Portland, Frank Cass, pp. 385-400.

SHEFFER Gabriel (2003) Diaspora Politics: At Home Abroad, Cambridge, Cambridge University Press.

SÖKEFELD Martin (2006) Mobilizing in transnational space: a social movement approach to the formation of Diaspora, Global Networks, 6 (3), pp. 265-84.

TISHKOV Valery (2003) Rekviyem po etnosy. Issledovaniye po sotsialno-uylturnoy antropologii, Moscow, Nauka. 


\title{
Post-Soviet Nation State as a Sponsor of Construction of the Ethno-National Diaspora: Azeri's Case
}

\author{
Sergey RUMYANSEV
}

The policy of constructing an Azeri ethno-national diaspora started to be intensively implemented in the early $21^{\text {st }}$ century. However, the formation of this purposeful policy was preceded by various events that demonstrated a constantly increasing interest on the part of the state in ethnic Azeris living in various countries of the world. This paper presents the results of the research into the process of formation of diaspora politics in Azerbaijan and also analyses the aims and specifics of the mechanisms of its implementation. The first one, the discourse practice, refers to the discursive effect of truth within the context of which "diaspora" acquires the features of a true, large, joint and homogenous community united by common goals. The second is the bureaucratic practice of the production of a hierarchically structured and co-subordinated organization structure. The bureaucratization of social networks of Azeris in the world occurs in 2000-th years, as a result of which an organizational structure of diaspora is constructed. This structure is uniform from local organisation up to those which represent all ethnic communities with "a Minister for the Diaspora" at its head and subordinated directly to the pan-Azeri president.

\section{L'État-nation postsoviétique comme soutien de la construction de la diaspora ethno-nationale : le cas azerbaïdjanais}

\section{Sergey RUMYANSEV}

La politique de constitution d'une diaspora ethno-nationale azeri commença à voir le jour de façon significative au début du XXIe siècle. Cependant, cette politique raisonnée fut précédée de différents évènements qui ont fait apparaître l'intérêt croissant de l'État pour les personnes d'ethnie azeri vivant dans différents pays du monde. L'auteur donne les résultats d'une recherche sur le processus de formation de politiques en faveur de la diaspora en Azerbaïdjan. Il en analyse aussi les buts et les mécanismes spécifiques. Le premier aspect renvoie aux effets de discours de vérité dans un contexte où la « diaspora » acquiert les traits d'une vaste communauté unifiée et homogène, unie par des buts communs. La seconde démarche relève de mécanismes bureaucratiques de co-production d'une structure organisationnelle hiérarchique. La bureaucratisation des réseaux sociaux azeris dans le monde intervient au cours des années 2000, avec pour conséquence la constitution d'une structure organisationnelle de la diaspora. Celle-ci est uniforme, du niveau local jusqu'à l'institution qui représente toutes les communautés ethniques, avec à sa tête un « Ministère des Diasporas », sous la tutelle du président pan-azeri.

\section{El estado-nación postsoviético como promotor de la construcción de une diáspora etnonacional: el caso azerbaiyano.}

\section{Sergey RUMYANSEV}

La política para la construcción de una diáspora etnonacional azerbaiyana ha sido implementada de manera intensiva a principios del siglo 21. Sin embargo, la elaboración de esta política fue precedida por varios acontecimientos que demostraron el interés cada vez más creciente del estado hacia la etnia azerbaiyana dispersada en diferentes países del mundo. Este informe presenta 
los resultados de la investigación sobre el proceso de elaboración de las políticas relativas a la diáspora en Azerbaiyán. Analiza también los objetivos y las especificidades de los mecanismos de su puesta en práctica. El primero, el ejercicio del discurso, remite al efecto discursivo de la verdad en el contexto del cual la diáspora adquiere los rasgos de une verdadera comunidad: amplia, homogénea y unida por un objetivo común. El segundo ejercicio concierne la burocratización de la producción de una organización co-subordinada y estructurada de manera jerárquica. La burocratización de redes sociales azerbaiyanas en el mundo empezó en los años 2000, generando la construcción de una estructura organizacional de la diáspora. Esta estructura tanto se refiere a una organización local como a una organización que representa a todas las comunidades étnicas. Tiene a su cabeza un ministerio de la Diáspora que depende directamente del presidente pan azerbaiyano.

\section{Постсоветское Национальное государство как спонсор конструирования этно-национальной диаспоры. На примере Азербайджанцев. Сергей РУМЯНЦЕВ}

Политика конструирования азербайджанской этно-национальной диаспоры стала активно реализовываться в период начала 2000-х годов. Формированию этой целенаправленной политики предшествовали различные события, которые демонстрировали постоянно возрастающий интерес государства к этническим азербайджанцам, живущим в разных странах мира. В данной статье представлены результаты исследования процесса формирования диаспоральной политики в Азербайджане, а также анализируются цели и специфика механизмов ее реализации. По мнению автора, механизмы реализации политического проекта конструирования диаспоры, в целом, сводятся к двум важнейшим и взаимосвязанным практикам. Первая - это дискурсивная практика. Рутинное, практически ежедневное напоминание в СМИ о деятельности «азербайджанской диаспоры», постоянные интервью этнических активистов, чиновников и различного рода общественных деятелей, рассказывающих о реализации проекта конструирования диаспоры. Вторая - это бюрократическая практика производства иерархически структурированной и соподчиненной организационной структуры. Единая от низовых организаций до тех, которые представляют уже все этнические общины структура, как бы построенная по принципу государственной вертикали власти представляется простой, понятной, прозрачной и удобной для контроля. Во главе этой структуры как бы стоит «министр диаспоры», подчиненный уже, непосредственно, всеазербайджанскому президенту. 


\title{
Revue européenne des migrations internationales
}

vol. $26-\mathrm{n}^{\circ} 3$ (2010)

Migrations en Asie centrale et au Caucase

Bayram Balci et Stéphane de Tapia

\section{Mouvements migratoires entre la Turquie et les Républiques turcophones du Caucase et d'Asie centrale : les impacts religieux}

\begin{abstract}
Avertissement
Le contenu de ce site relève de la législation française sur la propriété intellectuelle et est la propriété exclusive de l'éditeur.

Les œuvres figurant sur ce site peuvent être consultées et reproduites sur un support papier ou numérique sous réserve qu'elles soient strictement réservées à un usage soit personnel, soit scientifique ou pédagogique excluant toute exploitation commerciale. La reproduction devra obligatoirement mentionner l'éditeur, le nom de la revue, l'auteur et la référence du document.

Toute autre reproduction est interdite sauf accord préalable de l'éditeur, en dehors des cas prévus par la législation en vigueur en France.
\end{abstract}

revues.org

Revues.org est un portail de revues en sciences humaines et sociales développé par le Cléo, Centre pour l'édition électronique ouverte (CNRS, EHESS, UP, UAPV).

Référence électronique

Bayram Balci et Stéphane de Tapia, « Mouvements migratoires entre la Turquie et les Républiques turcophones du Caucase et d'Asie centrale : les impacts religieux », Revue européenne des migrations internationales [En ligne], vol. $26-n^{\circ} 3$ | 2010, mis en ligne le 01 décembre 2013, consulté le 02 janvier 2014. URL : http:// remi.revues.org/5225; DOI : 10.4000/remi.5225

Éditeur : Université de Poitiers

http://remi.revues.org

http://www.revues.org

Document accessible en ligne sur : $h$ ttp://remi.revues.org/5225

Ce document est le fac-similé de l'édition papier.

(c) Université de Poitiers 


\section{Mouvements migratoires entre la Turquie et les Républiques turcophones du Caucase et d'Asie centrale : les impacts religieux}

\section{Bayram BALCI ${ }^{1}$ et Stéphane de TAPIA ${ }^{2}$}

\section{AVANT PROPOS ${ }^{3}$}

T a Turquie et le monde turcophone font partie des régions les mieux couvertes

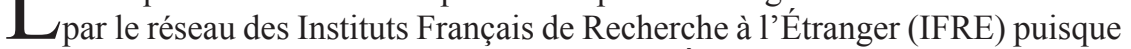
dans ce cadre fonctionnent l'IFEA (Institut Français des Études Anatoliennes) à Istanbul et l'IFEAC (Institut Français d'Études sur l'Asie centrale) à Tachkent, deux instituts qui ont très généreusement contribué à la formation des chercheurs français et plus généralement européens sur le vaste espace turcophone. Peuvent être partiellement adjointes les activités du Centre Franco-russe (Moscou), de l'IFRI (Institut Français de Recherches sur l'Iran, Téhéran), voire de la Délégation Française Archéologique d'Afghanistan (DAFA à Kaboul) puisque les marges de cet espace turcophone ont toujours été en contact avec les mondes russe et iranien étudiés par ces trois centres. La DAFA est bien plus spécialisée sur les périodes anciennes, de l'Antiquité à l'époque médiévale où les incursions turques ont

1 Ingénieur de recherche au CNRS, CERI, 56, rue Jacob, 75006 Paris ; balci_bayram@yahoo.fr

2 Directeur de Recherche au CNRS (Cultures \& Sociétés en Europe) ; stephane.detapia@misha.fr

3 Cet article est issu d'une contribution orale de Bayram Balci au Colloque des IFRE (Institut Français de Recherche à l'Étranger) à Paris et de visites de Stéphane de Tapia en Asie centrale et Azerbaïdjan dans le cadre d'un programme de l'Agence Nationale de la Recherche (Les Suds, aujourd'hui) auquel les deux auteurs collaborent. Intitulé « Caucase et Asie centrale postsoviétique, un autre Sud ?» (SudSov), ce programme cherche à mesurer les évolutions des républiques du sud de l'ancienne URSS et comprend un axe «migrations ». 
joué un grand rôle ${ }^{4}$. Une très grande partie des turcologues, travaillant sur la Turquie ou les républiques turcophones d'Asie centrale, ont effectué des séjours plus ou moins longs dans ces instituts.

Étant nous-mêmes passés par plusieurs de ces IFRE sous des statuts divers ${ }^{5}$, nous proposons aujourd'hui d'observer le phénomène migratoire entre la Turquie et les Républiques turcophones d'Asie centrale et du Caucase, appelé ici « monde turcophone », et d'y analyser plus particulièrement les flux religieux entre ces deux espaces, ou plus exactement comment le phénomène migratoire turc en Asie centrale s'est accompagné de flux religieux qui participent à l'heure actuelle à la recomposition de l'islam dans ces jeunes États. Nous utilisons à la fois les termes «migrants » et " expatriés » turcs sans toujours en montrer la distinction, car la présence turque dans l'ex-URSS est multiforme.

\section{INTRODUCTION}

Un exposé sur la Turquie et le monde turcophone nécessite au préalable une définition de ce "monde turcophone ", appelé aussi " monde turc " ou " turcique ». L'exercice est peu évident tant les concepts utilisés sont flous, polysémiques et tendancieux, puisque certains cachent des sensibilités politiques. Mais partons du constat que depuis le début des années 1990 la Turquie entretient des relations privilégiées avec des États issus de l'ex-URSS et qui partagent avec elle un héritage historico-géographique tant l'Asie centrale est considérée comme la patrie d'origine des Turcs avant leur migration en Anatolie par vagues successives, alors conquêtes effectuées par des nomades pastoraux. En effet, les Empires seldjoukide et ottoman qui verront naître la Turquie contemporaine étaient originaires d'Asie centrale. Ils ont été fondés par des populations nomades projetées depuis leurs steppes centrasiatiques à travers le Khorasan, terme général désignant aux époques antiques et médiévales l'Iran oriental, pour se fixer dans les terres anatoliennes alors byzantines.

Une bonne partie de la population turque actuelle possède des ascendances centrasiatiques, ce qui ne veut nullement dire qu'ethniquement tous les habitants de la Turquie contemporaine sont d'origine centrasiatique. Les populations anatoliennes et proche-anatoliennes déjà sur place ou déportées par les conquérants turco-ottomans ont été mélangées avec les nouveaux venus et constituent la base de la population de la Turquie actuelle. L'histoire plus récente des retraits successifs de l'Empire ottoman sur le déclin a également amené des rapatriés et réfugiés (en turc ottoman muhacir) très nombreux,

4 On parle encore dans les années 1930-1960 d'un Turkestan afghan, car les populations ouzbèkes et turkmènes y sont majoritaires. Historiquement, les dynasties turcophones, comme en Iran, jouent un rôle de premier plan, ne serait-ce que par l'émergence des Ghaznévides au Moyen Âge dont les souverains, anciens esclaves, sont d'origine turque (962-1039).

5 Bayram Balci a été doctorant, pensionnaire à l'IFEAC de 1996 à 1999, correspondant officiel de l'IFEA à Bakou entre 2002 et 2006, puis directeur de l'IFEAC de 2006 à 2010 ; Stéphane de Tapia a fréquenté l'IFEA comme étudiant, puis chercheur CNRS. Il a ensuite visité l'IFEAC et l'antenne IFEA de Bakou comme chercheur et membre de la Commission scientifique du ministère des Affaires étrangères responsable du «pôle TRIAC » (Turquie, Iran, Asie centrale) auquel s'est adjoint récemment le CRF de Moscou. 
originaires des Balkans, du Caucase, de Crimée, de la région Volga-Oural, voire plus récemment encore du Xinjiang et d'Afghanistan, qui ont encore accentué le caractère centrasiatique de la population, peut-être plus sur le plan idéologique que démographique (de Tapia, 1998). Mais malgré ce brassage, la référence en Turquie aux racines centrasiatiques reste constante, et ce malgré le puissant rêve européen qui anime les élites turques comme le reste de la population (Copeaux, 1997, 2000 ; Landau, 1981, 1995a et 1995b).

\section{CONSIDÉRATIONS GÉNÉRALES}

Pour entrer dans le vif du sujet, il s'agit pour nous d'analyser relations et phénomènes migratoires entre la Turquie et plusieurs pays de l'ex-URSS - Azerbaïdjan, Turkménistan, Ouzbékistan, Kirghizstan, Kazakhstan au moins partiellement turcophones -, mais aussi des pays limitrophes comme le Tadjikistan ou des régions autonomes de la Fédération de Russie (comme le Tatarstan), un vaste domaine géographique que les Turcs appellent monde turc (Türk dünyası) désigné en français par l'expression monde « turcophone » ou « turcique » et que la littérature anglo-américaine appelle Turkic ou Turkish World (Çağatay et Kuban, 2006).

La Turquie possède plusieurs points en commun avec ces États : appartenance ethnique, même origine géographique, histoire commune sur plusieurs siècles ${ }^{6}$, religion musulmane principalement sunnite, et surtout langue, même si des différences notables existent entre le turc anatolien et le kazakh, le kirghiz ou l'ouzbek (Bazin, 1986 ; Johanson, 2006). Depuis la fondation de la République turque par Mustafa Kemal en 1923 jusqu'à la dislocation de l'URSS en 1991, le discours en Turquie pour ces populations turciques était très peu élaboré et souvent l'apanage de cercles intellectuels de droite, anti-communistes et aux relents parfois fascisants. Cet intérêt pour les «Turcs de l'Extérieur » (Dlş Türkler) était dans une très large mesure alimenté par des immigrés turciques (Kazakhs, Ouïghours, Ouzbeks, Azéris) qui, fuyant le communisme, avaient trouvé refuge en Turquie, soit directement (Bezanis, 1994), soit indirectement après des passages par la Chine ou l'Afghanistan (Svanberg, 1989a, 1989b ; Salk, 1998). Peu connue en France, cette migration est en réalité très documentée.

À partir de 1990, du fait de la dislocation de l'URSS et de l'avènement sur ces cendres de républiques turciques ou turcophones indépendantes, ce discours à destination des Turcs de l'Extérieur renaît et l'on voit se développer un vaste intérêt pour ces nouveaux " pays frères », oubliés par la majeure partie de la population turque. Un contexte politique favorable (grâce au soutien des pays européens et des États-Unis pour que s'exerce une réelle influence turque dans la région supposée à tort proie facile de l'islamisme et de l'influence iranienne) et des autorités politiques centrasiatiques désemparées (car sommées soudainement de gérer une indépendance quasiment imposée) ont parmi d'autres facteurs

6 La visite de musées historiques à Istanbul, Ankara, Bichkek ou Tachkent, permet de s'en rendre compte, mêmes ancêtres déclarés, mêmes cartes, mêmes références religieuses, objets (archéologie, ethnographie) de factures très proches, mais les chemins se séparent progressivement après l'irruption mongole de l'Empire gengiskhanide et la fracture entre Ottomans sunnites et Séfévides chiites qui partageaient pourtant une origine ethnolinguistique identique, puis plus brutalement avec la prise en main par l’Empire russe puis la soviétisation de 1917 à 1990-91. 
d'ordre géopolitique éveillé en Turquie un rêve de grandeur, celui de constituer et diriger un monde turc uni et solidaire, mais aux contours jamais réellement définis. Ce contexte politique favorable, conjugué à l'esprit d'initiative des jeunes entrepreneurs anatoliens en quête de nouveaux marchés, a poussé des milliers de Turcs vers ces nouvelles républiques. Très rapidement, un vrai phénomène migratoire se développe entre la Turquie et ces républiques, porteur et vecteur de plusieurs discours idéologiques, principalement religieux.

\section{Tentative de description du phénomène migratoire turc en Asie centrale}

Il est difficile d'établir un panorama précis et détaillé, quantitativement et qualitativement du phénomène migratoire anatolien en Asie centrale et dans le Caucase, à savoir principalement en Azerbaïdjan, Turkménistan, Kazakhstan, Ouzbékistan, Kirghizstan. En l'absence de données statistiques quasi inexistantes, ou très parcellaires et souvent sujettes à caution, on ne peut fournir qu'une esquisse générale du phénomène (Balci et Buchwalter, 2001 ; Balci, 2005). Il s’agit généralement de petits commerçants, restaurateurs et responsables de petites et moyennes entreprises, très souvent dans le bâtiment et la construction, mais aussi l'agroalimentaire et la petite industrie (DİYİH passim) ${ }^{7}$. On trouve également des grandes entreprises turques de taille internationale, implantées dans ces pays, dans le domaine de la construction et du textile voire même des hydrocarbures, puisque la compagnie nationale turque de pétrole, TPAO est très active en Azerbaïdjan où elle est associée à la gestion du pétrole de la Caspienne, notamment à son acheminement dans le cadre de l'oléoduc dit BTC Bakou-Tbilissi-Ceyhan (de Tapia, 2008).

Les statistiques font donc défaut sur le nombre exact de migrants turcs dans ce pays, d'abord à cause du manque de services publics, hors Turquie, qui s'intéressent explicitement à cette question, mais aussi du fait qu'un grand nombre de migrants turcs qui travaillent dans ces pays n'ont pas d'enregistrement officiel, et continuent de résider sans aucun titre de séjour légal. Il existe toutefois des estimations qui permettent d'avancer le chiffre de 50000 Turcs en Azerbaïdjan, principalement à Bakou et dans son agglomération proche (Yunusov, 2009). Au Kazakhstan la présence migratoire turque serait beaucoup plus massive, dépassant les 100000 personnes, grâce à une économie locale très dynamique qui attire des travailleurs et des commerçants non seulement dans les deux capitales, Astana et Almaty, mais aussi dans les autres villes du pays, comme Shymkent et Türkistan, où se trouvent des universités turco-kazakhes, mais aussi dans des villes pétrolières comme Atyrau et Aktau sur la Caspienne (DİYİH, passim) ${ }^{8}$. Les nombreuses

7 On peut relever de nombreuses références en turc et en anglais traitant de la présence turque en Asie centrale, rapports officiels (ministères et établissements publics, ambassades, chambres de commerce, etc.) ou articles de chercheurs turcs ayant effectué une partie de leur carrière, stage, recherche, enseignement, dans différentes universités turques. Malheureusement, les données restent partielles, très générales et souvent redondantes.

8 Le rôle des Universités turques mériterait à lui seul une étude appropriée puisqu'elles ont été créées au Turkménistan (Mahtum Kuli), en Kirghizie (Manas et Alatoo), au Kazakhstan (Ahmet Yesevi et Suleyman Demirel), en Azerbaïdjan (Qafqaz). On y enseigne en anglais, russe, turc et langue nationale. Certaines sont liées à l'État turc directement, d'autres, privées au mouvement de Gülen. Les étudiants locaux y apprennent très vite un turc de qualité et ces universités ont généralement une bonne réputation, permettant aussi la projection vers l'Occident. 
entreprises turques importent parfois leur main-d'œuvre adaptée et qualifiée de Turquie, ce qui est parfois source de frictions entre immigrés turcs et travailleurs kazakhs ${ }^{9}$. Au Turkménistan, dont la capitale Achkhabad est en chantier permanent depuis au moins quinze ans (Fenot et Gintrac, 2005), les entreprises turques de BTP, concurrentes directes du groupe français Bouygues, font venir de Turquie des travailleurs turcs dont le nombre global dans le pays avoisine les 20000 , chiffre non négligeable compte tenu de la population du pays qui ne dépasse guère les 4 millions d'habitants. Au Kirghizstan, la présence migratoire turque est tout aussi visible, plus qu'en Ouzbékistan où la nature du régime karimovien (Poujol, 2005 ; Laruelle et Peyrouse, 2006) et les mauvaises relations entre Ankara et Tachkent ont réduit le nombre de Turcs installés en Ouzbékistan ces dernières années.

La migration s'effectue aussi dans l'autre sens, entre républiques turcophones et Turquie. Dans ce sens aussi, l'immigration dite économique liée au commerce et au travail prime. Les chantiers turcs comptent de plus en plus de travailleurs de ces républiques principalement d'Azerbaïdjan et d'Ouzbékistan, mais c'est surtout le commerce qui attire des Centrasiatiques et des Caucasiens en Turquie, notamment le secteur du textile et du cuir. Il faut aussi mentionner l'existence d'une immigration centrasiatique intellectuelle, notamment des chercheurs et des enseignants, des professeurs de musique, et plus largement des comédiens, danseurs et artistes qui viennent faire carrière en Turquie. Enfin, on ne saurait oublier la présence de plusieurs milliers d'étudiants de ces Républiques, inscrits dans les universités turques, avec souvent le soutien financier du gouvernement d'Ankara. Il y aurait à l'heure actuelle entre 2000 et 3000 étudiants de chaque république en Turquie, à l'exception de l'Ouzbékistan qui freine ses relations avec la Turquie.

\section{Les flux idéologiques véhiculés par cette migration à double sens}

Le lien entre migrations, migrants et diffusion d'idées religieuses est une réalité bien connue qui a souvent été étudiée dans les sciences sociales. En cela, notre étude et notre terrain n'ont rien d'original, mais apportent leur pierre à l'édifice général. Ce lien entre migration et circulation d'idées religieuses est par ailleurs complexe, dynamique et interactif. L'immigration peut induire indirectement un message religieux et sa diffusion par la migration, et inversement, le discours religieux peut être la raison même de la migration, c'est-à-dire le résultat de la volonté missionnaire et pèlerine du candidat à la migration. Ne dit-on pas que le Prophète Mohammad, a migré de la Mecque à Médine pour exporter sa foi et la diffuser par la suite dans la péninsule arabique et au-delà, modèle repris par des millions de réfugiés et rapatriés dits muhacir en ottoman, mohajjer dans l'aire irano-pakistanaise ${ }^{10}$. Cette remarque vaut pour toutes les idées, religieuses, mais pas

9 En octobre 2006, un violent incident a opposé travailleurs kazakhs et turcs employés par la firme turque ENKA à la suite de tensions croissantes sur un chantier pétrolier de l'entreprise américaine Chevron. Les Turcs ont compté plus de 150 blessés, dont certains gravement atteints, qu'il a fallu rapatrier en Turquie par avion.

10 De la racine hajr (migration) qui a donné notre Hégire historique, les termes muhacir / mohajjer désignent ces migrants ayant décidé (ou ayant été forcés) de migrer du Dar'ül Harb, terre de conflit, au Dar'ül Islam, terre d'islam. Certains islamistes du «Londonistan » s'autodésignent comme Mohajjirun, les migrants, par référence directe à l'Hégire. 
seulement. Par ailleurs, le message véhiculé peut être en cours de route altéré et prendre des formes inhabituelles, hybrides.

Dans le cas des relations migratoires entre la Turquie et l'Asie centrale, on observe le même phénomène, à savoir que plusieurs courants idéologiques ont été provoqués ou ont provoqué eux-mêmes un mouvement migratoire porteur de messages idéologico-religieux. De fait, le premier discours a été nationaliste et racial, mais n'a pas obtenu tout le résultat escompté (Türk, 2009), ce qui nous permet de l'analyser plus rapidement et de passer à l'examen du principal courant idéologique parti de Turquie, dans les valises des migrants turcs, pour souder ou ressouder cette vaste Asie centrale turcophone à la Turquie et à ses différents courants islamistes.

Jusqu'à la chute de l'Union soviétique, les seuls en Turquie à avoir un discours et un intérêt pour les populations turciques d'URSS étaient les courants nationalistes turquistes, de tendance " droite réactionnaire ». Leur discours prône la grandeur de la race turque et ils vouent un véritable culte au foyer originel des Turcs, l'Asie centrale et la Sibérie, appelé Touran dans la phraséologie nationaliste. Ce courant, au demeurant très hétéroclite et traversé par des clivages de diverses natures, est souvent animé par des réfugiés azéris, kazakhs, ouzbeks, tatars, ouïghours ayant fui le communisme soviétique ou chinois et établis en Turquie où ils créent des associations, des revues et des clubs où la référence à ce Turkestan perdu est constante. Ces courants nostalgiques de la Patrie perdue rêvaient de la dislocation de l'Union soviétique qui permettrait à leur pays de retrouver l'indépendance et la liberté (Landau, 1991 ; Copeaux, 1997).

Au moment où l'Union soviétique se disloque, ces courants crient victoire et estiment proche le moment où le rêve deviendra réalité avec la création d'un monde turc uni allant de la Turquie jusqu'à la frontière chinoise. Croyant dur comme fer en cette grandeur bientôt retrouvée, certains individus ou courants migrent dans ces pays « enfin libérés du communisme » et en cela ils sont encouragés par un contexte géopolitique international favorable et des autorités politiques qui multiplient les déclarations de retrouvailles entre frères turcs, dans un même monde qui va, selon le président Demirel, «de l'Adriatique à la Muraille de Chine » (Balci, 1999, 2001).

Or, ce rêve s'est vite heurté aux dures réalités du terrain, à savoir la surdité des « frères » turcs à ce discours panturquiste et souvent déconnecté des réalités, bien que des tentatives aient été menées, vite contrées par les gouvernements en place (Türk, 2009). Ces migrants, partis faire du commerce ou des études dans ces pays frères ont constaté avec amertume le décalage entre leurs rêves et la réalité disparate faite de différences de culture, de mentalité et même de langue entre ces peuples pourtant frères. Tirant la conclusion de l'inexistence d'un monde turc, la plupart ont renoncé à leur discours panturquiste et ont bien été obligés d'apprendre le russe, langue de communication par excellence de la région pour pouvoir communiquer avec leurs « frères ».

Une exception mérite tout de même d'être mentionnée et elle concerne l'Azerbaïdjan, où ce discours de retrouvailles entre frères de Turquie et d'Azerbaïdjan n'a pas complètement échoué. Plus que les autres peuples turcophones, les Azéris - la société comme le pouvoir - revendiquent cette fraternité turque avec les autres peuples turco- 
phones, mais surtout avec la Turquie et qui, pour des raisons géopolitiques complexes notamment liées à la question arménienne, ont une vision de la politique régionale convergente avec la Turquie. Et à l'heure actuelle dans les rues de Bakou comme dans les bureaux officiels, on entend très régulièrement, pour qualifier les relations turco azéris, le slogan «Une même Nation, Deux États » (Bir Millet, iki Dövlet). Dans ce cas précis de discours panturquiste entre la Turquie et l'Azerbaïdjan, les migrants en ont été à la fois les porteurs, de même qu'ils ont été portés par ce rêve dans leur choix de migration, même si les considérations économiques ont été aussi très présentes dans le choix et la stratégie migratoires.

\section{LES COURANTS RELIGIEUX AU CENTRE DE CETTE DYNAMIQUE MIGRATOIRE}

Plus que les courants nationalistes, ce sont les mouvements islamistes qui ont le plus bénéficié de l'établissement des liens avec la Turquie. Se sont déversées, dans la vaste Asie Centrale, dans le Caucase et jusque dans les régions musulmanes de la Fédération de Russie, plusieurs vagues successives de missionnaires anatoliens venus islamiser ou réislamiser ces terres fraîchement libérées de l'idéologie communiste et de la propagande soviétique antireligieuse. Pour la plupart ce sont des groupes privés, et en principe indépendants des acteurs étatiques, qui investissent le champ religieux. Mais il convient au préalable d'expliquer l'attitude des États face à l'islam en cette période de fortes turbulences pour tous les régimes issus de l'ex-URSS. Car, même si les flux islamiques entre la Turquie et l'Asie centrale ont été l'œuvre de mouvements privés et fortement liés au phénomène migratoire, les acteurs étatiques n'ont pas été indifférents et leurs perceptions de la situation à l'époque n'ont pas été sans effet sur les résultats des actions missionnaires privées déployées dans ces pays.

Pour la Turquie de ce début des années 1990, comme il a été souligné précédemment, l'espace turcophone du Caucase et d'Asie centrale, représente un nouvel espace d'investissements en termes politiques, économiques et culturels. Outre la mise en place d'un vaste programme éducatif qui permet à des milliers de jeunes étudiants turcs d'étudier en Asie centrale et à des milliers de jeunes centrasiatiques de poursuivre des études en Turquie, l'État turc a aussi, et c'est cela qui est audacieux pour une République laïque et séculière, mis en œuvre un véritable mécanisme de coopération islamique avec ces États, principalement par le biais de ses ambassades où est affecté un attaché aux affaires religieuses chargé d' " exporter » la vision officielle turque de l'islam et de sa place dans la société (Yazgan, 2003). C'est cet attaché qui, par exemple, coordonne les programmes d'échanges entre la Turquie et son pays d'affectation en matière de formation de nouveaux imams et de restauration ou de construction de mosquées. Dans certains cas, il a aussi prise sur les activités religieuses déployées par les migrants turcs dans le pays d'accueil.

Pour les États nouvellement indépendants, la question du contrôle des flux religieux ne se pose pas dans les premières années de leur indépendance. Bien que crispées et anxieuses face à une indépendance davantage imposée par la chute de l'Empire soviétique à partir de Moscou que de la périphérie, les autorités politiques, toutes issues de l'ancien système, se complaisent tout de même dans une euphorie illusoire, celle de l'ouverture vers le monde et de l'établissement de contacts avec des États et de peuples 
longtemps tenus à distance par le pouvoir central soviétique. Dans ce contexte, la circulation des idées et des hommes se développe dans un enthousiasme positif partagé par tous. Des contacts sont établis avec l'extérieur sur la base de solidarités ethniques et/ou religieuses. Un exemple assez révélateur qui relève à la fois de la question migratoire et du phénomène religieux n'est autre que le hadj, le pèlerinage à La Mecque. Très limité pendant la période soviétique, il connaît une forte croissance dès 1992. C'est dans ce cadre bon enfant d'ouverture vers l'extérieur que se développe la rencontre entre idées religieuses en Asie centrale, transportées par des migrants de différentes provenances géographiques, et une population locale avide de contact avec un étranger proche dont le modèle est la Turquie à la fois semblable et différente.

Plusieurs mouvements, organisations, tendances, ou phénomènes confrériques ou néo-confrériques turcs s'intéressent alors à cette région du monde dès cette période où les liens migratoires commencent à s'établir entre la Turquie et l'Asie centrale (Balci, 2003). Nous nous attarderons plus particulièrement sur quatre d'entre eux : le mouvement de Sait Nursî dont les disciples sont appelés Nurcu ; les enseignants éducateurs de Fethullah Gülen qui est un des héritiers spirituels de Sait Nursî ; les disciples de Süleyman Tunahan, une grande figure islamique qui a marqué la Turquie des années 1970 et dont les disciples, nombreux en Asie centrale, sont communément appelés les Süleymancı; des sous-groupes de la grande confrérie nakchibendie qui, à partir de leur base arrière d'Istanbul, ont développé un vaste programme d'assistance humanitaire teinté d'intentions islamiques dans tout l'espace musulman de l'ex-URSS, principalement en Asie centrale. Les frontières entre ces mouvements sont souvent mouvantes et des transfuges peuvent être observés entre groupes, surtout quand les besoins économiques ou les opportunités d'expatriation le rendent nécessaire.

Les Nurcu furent parmi les premiers à saisir l'opportunité offerte par l'ouverture de l'ex-URSS pour s'exporter en Asie centrale. Le terme nurcu renvoie à Sait Nursî, une autorité religieuse née à l'est de la Turquie (1876-1960) et qui occupe une place essentielle dans l'histoire religieuse de la Turquie moderne. Émergeant dans un contexte de fin d'Empire, ce mouvement est avant tout piétiste et cherche à lutter contre la perte de foi et de pratique au sein de la population turque de l'époque, très marquée par les réformes séculières de Kemal Atatürk. Conservateur, mais à la fois nationaliste turc et islamiste modéré, ce mouvement n'est pas politique, mais cherche avant tout à octroyer à l'islam une place centrale au sein de la société turque et dans l'espace public. Interdit et condamné par le régime kémaliste, au lendemain du passage en Turquie à un système politique plus démocratique, notamment dans les années 1950, l'histoire de la confrérie oscille entre bannissement et existence légale. À la mort du père fondateur, le mouvement se scinde en plusieurs tendances, mais toutes sont caractérisées par une pratique militante qui cherche à desserrer le contrôle excessif de l'État sur la religion en Turquie.

Au début des années 1990, différentes tendances issues de ce mouvement s'exportent en Asie centrale, très souvent par le biais du petit commerce et du travail dans les grandes entreprises turques de construction. Le discours religieux des membres de ces différentes associations ou mouvances spirituelles marquées par l'héritage de Nursî n'a rien de révolutionnaire. La plupart du temps leur action se limite à organiser, dans des madrasas semi-officielles ou simplement dans des maisons ou appartements collectifs, des 
lectures de l'œuvre maîtresse de leur père spirituel, la Risale-i Nur. Ce texte, «Épître de la Lumière ", n'est autre qu'une exégèse du Coran, écrite dans une langue plus ottomane que turque, à tel point que sans un dictionnaire ou l'assistance d'un maître, sa lecture ou sa compréhension s'en trouve quasiment contrariées. Semi-légales, les activités de ces migrants turcs sont selon les pays sous étroite surveillance. Le mouvement comptait énormément sur les possibilités d'action prosélyte en Ouzbékistan, pays le plus peuplé et le plus musulman de la région. Or, les relations diplomatiques tendues entre Ankara et Tachkent, et surtout le manque flagrant de libertés religieuses ont considérablement freiné son implantation dans le pays.

Issu de ce même mouvement fondé par Sait Nursî, l'organisation de Fethullah Gülen, un gourou à la mode anatolienne, mérite une place particulière dans l'analyse tant son action et son impact sont considérables dans l'exportation d'un islam turc en Asie centrale et dans le Caucase. Le mouvement, doté d'une identité à géométrie variable, tantôt néo-confrérie, tantôt mouvance ou même nébuleuse est fortement lié à la personnalité de son fondateur Fethullah Gülen, un des nombreux héritiers de Sait Nursî. Aux contours imprécis, cette mouvance religieuse s'est formée sur une longue période, entre les années 1970 où, dans la région d'Izmir, Gülen et ses proches créent plusieurs associations culturelles et éducatives, et les années 1990, décennie caractérisée en Turquie par une remarquable entrée dans la libéralisation économique permettant l'émergence de centaines d'entreprises économiques très fortement structurées autour de valeurs islamiques. À partir d'un noyau d'associations rassemblant des parents d'élèves et des éducateurs, la mouvance gagne la confiance de milliers d'entreprises et d'entrepreneurs et parvient à bâtir un pseudo « holding » sans personnalité juridique distincte et précise, mais disposant d'un puissant levier économique et financier dépassant les frontières de la Turquie.

Le contenu idéologique de la tendance est difficilement identifiable, car Fethullah Gülen se veut apolitique et ses prédications et discours empruntent à des registres très variés : mystique soufie, nationalisme turc, islam progressiste et moderniste, œcuménisme islamo-chrétien entre autres. À la fin des années 1980, le mouvement est à son apogée et commence même à inquiéter les gardiens du dogme kémaliste, si bien que l'Armée et les milieux séculiers craignent sa possible transformation en force politique, après une longue période de gestation dans un cadre éducatif et associatif. Cette fin de décennie est aussi celle de l'établissement de liens divers entre la Turquie et l'ex-URSS, une opportunité, une aubaine pour les entreprises proches de Gülen désormais trop puissantes pour se limiter au territoire proprement turc et à la recherche de nouveaux marchés commerciaux et de nouvelles terres de prédication.

C'est ainsi que des petits commerçants, des travailleurs, des éducateurs et parfois même des entreprises de taille internationale, se trouvant plus ou moins dans la sphère spirituelle de Fethullah Gülen, se sont exportés en Asie centrale pour y travailler et propager par la même occasion les idées de leur maître. Ces disciples de Gülen, les fethullahçı, s'illustrent notamment par la création et l'animation de tout un réseau éducatif de lycées et des centres de langue, voire même des centres de préparation aux concours universitaires. L'enseignement y est séculier et laïc en toute conformité aux normes nationales héritées de la période soviétique ; aucun enseignement coranique ou islamique n'est 
dispensé dans les lycées et aucune littérature islamique n'y est propagée. Mais, et c'est là toute la subtilité de leur méthode, sans cours de religion dans des écoles prétendument laïques, le mouvement parvient tout de même à véhiculer la pensée de Fethullah Gülen, prônant un islam à la fois turc national, en bons termes avec l'État en place, marqué par le soufisme et dépourvu de toute ambition politique. Dans chaque pays d'Asie centrale, les dizaines d'écoles fondées par le mouvement travaillent en symbiose avec des entreprises turques implantées dans le pays (Balci, 2003). Le corps enseignant est constitué de jeunes professeurs, tout juste sortis des universités turques, partis en Asie centrale pour des motivations à la fois économiques et spirituelles, et pour diffuser une forme évoluée d'islam dans une région considérée comme le pays d'origine des ancêtres. Comparables, toute proportion gardée, aux jésuites partis d'Europe occidentale pour propager la bonne parole chrétienne à travers le monde, les cadres supérieurs de Gülen avouent volontiers s'inspirer de ce phénomène missionnaire pour, à leur tour, exporter leur foi et leur culture dans de nouvelles contrées.

Un autre mouvement turc, moins structuré et moins riche, mais avec la même logique de dissémination de migrants anatoliens en Asie centrale et dans le Caucase, tente à sa manière de contribuer au flux spirituel qui à partir de la Turquie vient irriguer les friches postsoviétiques. Il s'agit des disciples d'un autre grand intellectuel musulman turc, Hilmi Süleyman Tunahan, appelés les Süleymancı, qui diffusent, plus ouvertement que les Fethullahçı, la philosophie islamique de leur maître. Le mouvement en Turquie du temps de son fondateur s'était fait remarquer par ses querelles constantes avec la Diyanet, Présidence des affaires religieuses sous tutelle du Premier ministre, la plus haute instance officielle de gestion de l'islam turc, qui voulait imposer son monopole sur la gestion de l'islam en Turquie, ce que refusaient certains mouvements privés dont celui de Süleyman Tunahan. Le mot d'ordre du mouvement a toujours été et demeure " permettre à chaque musulman de pouvoir lire le Coran en arabe ». Leitmotiv qui n'a guère changé puisqu'en Turquie comme dans leurs pays d'expatriation les militants du mouvement placent au centre de leurs activités et de leurs préoccupations la fondation d'écoles coraniques, où la priorité absolue est de permettre aux jeunes générations l'accès au Coran en langue arabe. L'islam promu par ces expatriés turcs véhicule aussi une certaine idéologie turquiste (Bozay, 2005), du moins promeut une image positive de la Turquie (Ateş, Karakaş et Ortayl1, 2005). Dans chaque pays où le mouvement est implanté, il entretient de bonnes relations avec les instances officielles de l'islam et les organisations islamiques modérées.

Autant que les autres mouvements turcs, cette mouvance s'appuie sur les commerçants et petits entrepreneurs turcs pour se développer dans ces pays. Elle a ouvert dans tous les pays de la région, sauf en Ouzbékistan et au Tadjikistan, de modestes madrasas entièrement financées par des fondations et associations implantées en Turquie et des entreprises turques présentes en Asie centrale et dans le Caucase. Régulièrement des nouveaux cadres religieux arrivent de Turquie et certains jeunes étudiants sont envoyés dans les fondations privées Süleymancı partout en Turquie pour y perfectionner leur formation. Cette mouvance s'est surtout implantée dans la région caucasienne, en Azerbaïdjan et en Géorgie, notamment dans la province d'Adjarie. Particularité de leur présence dans cette région, la mouvance utilise les descendants de la diaspora caucasienne en Turquie pour diffuser leurs idées. En effet, il existe en Turquie de très nombreuses associations de descendants des mouvements diasporiques caucasiens, qui avaient trouvé refuge en 
Turquie ottomane pour fuir la domination russe. Depuis la fin de l'URSS, une partie des échanges, de tout genre, entre la Turquie et le Caucase est le fait de ces enfants de la diaspora. Ainsi, une bonne partie des missionnaires envoyés dans ces contrées ont des origines caucasiennes.

Les Nakchibendis constituent le quatrième et dernier groupe turc important à s'être beaucoup intéressé à l'Asie centrale. Ainsi des organisations nakchibendies ont développé, en s'appuyant sur nombre de volontaires turcs au départ, plusieurs activités pour renouer le contact avec les nakchibendis de l'ex-URSS et pour apporter leur contribution au processus de réislamisation de cette région. La nakchibendiyya, du nom de Bahaduddin Nakchibendî, un mystique du XVe siècle né à dix kilomètres de Boukhara, est une confrérie soufie qui à partir de ce foyer centrasiatique s'est diffusée dans tout le monde musulman, principalement en Inde, dans le Caucase et en Turquie. Prônant un islam mystique modéré, mais plus ou moins politisé selon les époques et les lieux, cette confrérie occupe une place essentielle dans le champ islamique de Turquie où elle est incarnée par plusieurs branches animées par d'importantes autorités religieuses. Dans leur ensemble, quelque soit leur branche, les nakchibendis de Turquie ont toujours entretenu une forte nostalgie pour la patrie du fondateur de l'ordre et l'on sait qu'au début des années 1990, Turgut Ozal, Premier ministre et président de la République par la suite, par admiration pour cet ordre qui lui avait apporté un soutien politique et électoraliste non négligeable, a généreusement contribué à la restauration du mausolée et facilité les premiers contacts entre Nakchibendis de Turquie et leurs confrères d'Ouzbékistan.

Forts de ces soutiens, les disciples de cette confrérie ont également utilisé le flux important des travailleurs turcs en Asie centrale pour mettre leur entreprise mystique à l'épreuve du contact avec la patrie de la nakchibendiyya. Mais surtout, dans le cas de cette confrérie, c'est davantage le pèlerinage sur la tombe de Bahauddin Nakchibend à Boukhara qui a permis de recréer des liens entre ses disciples de Turquie, d'Asie centrale et d'ailleurs. Un véritable tourisme religieux, impensable pendant la période soviétique, s'est développé à partir de la Turquie d'où des compagnies proposaient dès 1990 un parcours incluant Tachkent, Boukhara, Ghijduvan et Samarkand, où se trouvent d'importants sites liés à la nakchibendiyya ou à d'autres ordres, comme la yasaviyya dont le berceau se trouve dans la ville aujourd'hui kazakhe de Turkestan (Balci, 2001).

Paradoxalement, c'est moins dans la patrie originelle de la nakchibendiyya, en Asie centrale, que dans les villes du Caucase, en Azerbaïdjan et en Géorgie notamment, que les nakchibendis turcs ont été les plus influents et les plus à même de proposer leurs " services ", à savoir un enseignement islamique en langue turque des œuvres caritatives, la restauration ou reconstruction de certaines mosquées ayant souffert par le manque d'entretien pendant la période soviétique, etc. En Azerbaïdjan, plus massivement dans les régions sunnites que chiites, la branche nakchibendie, liée à la fondation Mahmut Hudayi et animée à l'heure actuelle par le leader nakchibendis Osman Nuri Topbaş et originaire de la ville d'Üsküdar, dans l'agglomération d'Istanbul, gère une demi-douzaine d'établissements religieux dans lesquels se forment les nouvelles élites musulmanes du pays (Balci et Motika, 2007). Comme dans le cas des autres mouvements, d'importants échanges et de va-et-vient ont lieu entre la Turquie, et le pays d'implantation, pour permettre le transfert de savoir dans les deux sens. Car si les Turcs cherchent à exporter leur vision de l'islam, ils 
reçoivent en retour, à un moindre degré certes, des influences islamiques issues des traditions de ces pays-là et plus particulièrement d'Asie centrale d'où sont originaires nombre d'étudiants venus en Turquie pour leurs études universitaires.

\section{Comment mesurer l'impact de ces flux religieux sur les sociétés d'Asie centrale et du Caucase ?}

Bien que déployé sur une courte période, de 1991 à nos jours, le phénomène migratoire en Asie centrale et dans le Caucase a particulièrement affecté l'espace urbain et les sociétés locales. La présence turque expatriée ou immigrée, dans la sphère économique, industrielle, commerciale ou bien culturelle, éducative et religieuse est particulièrement visible et fait partie des réalités quotidiennes des grandes villes de Bakou à Almaty en passant par Achkhabad, Tachkent, Bichkek et Astana (Güler, 2007) ${ }^{11}$. La croissance des mariages mixtes favorise un certain brassage et accentue le phénomène migratoire définitif de ceux qui au départ n'étaient qu'expatriés. Même si en termes de gros capitaux la présence économique turque est plus faible que celle des grandes puissances économiques mondiales comme l'Allemagne ou les États-Unis, les entreprises étrangères de taille moyenne sont très souvent originaires de Turquie. Sans même que l'enseignement du turc soit particulièrement présent dans les écoles et les universités, le turc anatolien est connu voire pratiqué par de plus en plus de gens, grâce à la forte et constante présence turque depuis plus de quinze ans. La proximité linguistique entre le turc anatolien et les langues turciques locales facilite aux Turcs l'apprentissage de l'ouzbek, du kazakh mais inconsciemment ou consciemment c'est le turc qu'ils imposent à leurs partenaires locaux. Dans les grandes entreprises turques en tout cas, la langue de travail est le turc, devant la langue officielle locale (kazakhe, ouzbek, kirghize, turkmène, etc.) et le russe.

En termes religieux, l'impact de la migration turque en Asie centrale est réel, bien que difficile à évaluer avec précision, et est souvent tributaire de la nature des relations entre la Turquie et chacun des États de la région. De même, le degré de liberté qui y prévaut, notamment en matière de liberté religieuse, détermine le degré d'impact des missionnaires turcs en Asie centrale.

Dans un souci de simplicité, procédons à un bilan par pays, et commençons par l'État le plus proche, à tous points de vue, de la Turquie, l'Azerbaïdjan où les migrants turcs ont été parmi les premiers à s'implanter dès 1990, dans certaines villes avant même les religieux iraniens pourtant très proches de ce pays (Balci et Motika, 2007). En convergence avec l'action de l'État turc qui leur a facilité la tâche, et pour une série de raisons à la fois politiques et géopolitiques (la question arménienne notamment), les migrants turcs affiliés à l'une ou l'autre des organisations religieuses évoquées ici ont déjà formé des centaines de membres des nouvelles élites religieuses à travers tout le pays. Au sein même de l'appareil de gestion de l'islam, la direction des affaires religieuses, mais aussi le comité d'État pour les affaires religieuses, on trouve de nombreux cadres formés sur place par les fondations caritatives turques. En cela, les organismes privés, originaires d'un pays

11 Écrit par un cadre d'entreprise privée qui a effectué une partie non négligeable de sa carrière dans la région, cet ouvrage est un plaidoyer pour une approche plus pragmatique et plus réaliste des sociétés locales par les entrepreneurs et surtout les pouvoirs publics turcs.

REMI 2010 (26) 3 pp. 133-152 
laïc et séculier comme la Turquie, ont été plus performants que les missionnaires envoyés par la République islamique d'Iran. Cette plus grande performance des mouvements turcs montre à quel point le contexte politique est important, y compris quand il s'agit de la circulation des idées religieuses.

Le Turkménistan est aussi un cas assez intéressant dans cette analyse des flux religieux turcs véhiculés en Asie centrale. Malgré la fermeture de son régime et son manque de libertés religieuses, le Turkménistan a été un terrain de prédication particulièrement bien investi par les mouvements islamiques turcs, mais aussi par l'État turc dont l'attaché aux affaires religieuses près l'ambassade de Turquie gère depuis plus de quinze ans de vrais programmes de coopération islamique, notamment en termes de formation grâce à la création d'une faculté de théologie. Une visite dans la plupart des villes de ce pays, avec à chaque fois des haltes importantes dans les principales mosquées, nous a permis de mesurer l'importance de cet impact turc sur l'islam turkmène. La plupart des imams formés depuis l'indépendance du pays, ont un rapport direct avec la Turquie : ils ont été formés soit par l'État turc, soit par des fondations privées qui déploient leurs activités dans le pays d'une manière pas toujours légale d'ailleurs.

L'Ouzbékistan est un cas plus complexe à analyser, tant les relations avec la Turquie se sont dégradées depuis le milieu des années 1990, après avoir été si chaleureuses et cordiales durant les premières années de l'indépendance ouzbèke. Pour les résumer à l'extrême, les relations ouzbéko-turques ont souffert du fait que l'opposition au régime de Tachkent, les partis Erk et Birlik, se sont installés dès 1994 en Turquie où ils ont bénéficié $\mathrm{du}$ soutien de certains milieux nationalistes turcs, sans parvenir toutefois à gagner la confiance des autorités officielles turques (Türk, 2009). En revanche, ils étaient particulièrement populaires parmi les milliers d'étudiants ouzbeks envoyés par Tachkent en Turquie, si bien que rapidement cette opposition ouzbèke a inquiété le régime de Karimov qui, de tout temps suspicieux à l'égard de toute idée subversive, a fait rapatrier la quasitotalité des étudiants ouzbeks de Turquie et gelé ses relations avec la Turquie.

En dépit de la méfiance des Ouzbèks qui ont toujours étroitement surveillé toute influence extérieure, certains mouvements islamiques turcs se sont bien implantés dans les principales villes ouzbèkes tout au long des années 1990. Le mouvement de Gülen était présent massivement dans tout le pays, mais à partir de septembre 2001, le pays, sombrant dans la paranoïa a mené une véritable chasse aux sorcières contre les Turcs, accusés de subversion. Toutes les écoles ont été fermées du jour au lendemain ; certains entrepreneurs liés à ces écoles ont été expulsés, et des Ouzbeks compromis avec ces établissements ont écopé de peines de prison. Cette épuration n'a pas complètement réussi à éradiquer les idées déjà véhiculées par ces missionnaires turcs puisqu'en avril 2009 plusieurs membres ouzbeks soupçonnés d'appartenir à la mouvance nurcu et de propager leurs idées subversives ont été arrêtés et mis en prison. 
Le Kirghizstan, mais surtout le Kazakhstan avec ses pharaoniques projets de construction dopés par la manne pétrolière, attire de nombreux entrepreneurs étrangers ${ }^{12}$. Parmi eux, des Turcs originaires de la Turquie profonde, de cette Anatolie moralement conservatrice, mais économiquement très dynamique. Les mouvements islamistes turcs évoqués, Nurcu, Fethullahçı et autres, trouvent un terrain particulièrement propice à leurs activités. Ils sont en cela indirectement encouragés par les autorités kazakhes qui y voient un moyen de favoriser le renouveau de la culture kazakhe dont les Turcs se font les porte-parole sur place, dans un contexte démographique où l'élément slave est encore très important puisqu'environ $40 \%$ de la population est russe ou russophone. Pour les autorités kazakhes, la présence turque fait contrepoids à la forte composante slave de la population. Pour les missionnaires turcs, avec une université à Almaty, trente-cinq établissements de niveau lycée répartis dans tout le pays, et une vingtaine de modestes madrasas gérées par les Süleymancl, le Kazakhstan est une terre de conquête, un vaste territoire vierge, peuplé d'autant de Russes que de Kazakhs, tous très perméables à la propagande religieuse qui y faisait défaut depuis longtemps. Aujourd'hui, au Kirghizstan comme au Kazakhstan, tous les cadres religieux sont redevables d'une manière ou d'une autre aux autorités religieuses turques ou à ces mouvements religieux turcs confrériques privés. Tant et si bien que naturellement l'islam turc sous toutes ses formes exerce une influence considérable sur l'establishment religieux local, voire même sur les nouvelles élites politiques souvent tentées de s'inspirer de la Turquie pour bâtir une nouvelle nation où le facteur religieux n'est pas totalement marginalisé.

L'islam ou plutôt les islams de Turquie ont une aura bien plus large et leur influence dépasse le cadre des républiques turcophones puisque le Tadjikistan, les régions turcophones de la Fédération de Russie, notamment le Tatarstan et le Bachkortostan, sont également des terrains importants pour leur action missionnaire appuyée par le soutien des entreprises et travailleurs turcs (Korkut, 2010).

\section{CONCLUSION}

Pour une multitude de raisons, politiques, économiques, identitaires, sécuritaires, aucun État moderne ne peut rester indifférent face au phénomène migratoire, qu'il s'agisse d'immigration et d'émigration, d'entrée sur son territoire de citoyens étrangers ou de sortie de ses propres citoyens vers un autre pays. Source d'instabilité, mais aussi de création de richesse (apport de devises) et d'instrumentalisation politique (création de groupe de pression dans le pays d'expatriation), le fait migratoire a souvent fait l'objet de politiques publiques dans plusieurs pays du monde. Ainsi la Turquie, comme d'autres, veille scrupuleusement à la nature de ses relations avec ses ressortissants, d'autant plus qu'elle a une certaine expérience en la matière puisque depuis les années 1960, des millions de

12 Voir par exemple le site de l'Union des Entrepreneurs de Turquie (Türkiye Müteahhitler Birliği), http://www.tmb.org. Les entreprises turques du secteur ont pris une grande part de la rénovation d'Almaty, de la construction de la nouvelle capitale kazakhstanaise, Astana, et se sont spécialisées dans les infrastructures aéroportuaires et routières, touristiques (grands hôtels), sanitaires (hôpitaux), éducatives (établissements secondaires, universités), industrielles. Nombreuses informations disponibles sur les sites ministériels, diplomatiques, économiques, turcs, mais souvent redondants. 
travailleurs turcs ont immigré en Europe, Allemagne, France et autres pays où la communauté turque a un poids politico-religieux qui ne peut la laisser indifférente.

En Asie centrale et dans le Caucase, elle veille plus particulièrement à ce que la présence turque ne détériore pas ses relations avec les États de la région. Dans certains cas, elle cherche même, comme d'autres États le font ailleurs, à utiliser ses ressortissants, engagés voire embrigadés dans les actions d'associations ou mouvements informels privés, pour les mettre au service de sa politique extérieure.

S'agissant des rapports entre l'État et les mouvements missionnaires turcs en Asie centrale et dans le Caucase, la République turque, séculière et laïque maintient une attitude pour le moins pragmatique. Tandis qu'en Turquie même les relations entre l'État et les organismes religieux peuvent être tendues, en Asie centrale, pour des raisons de convergence d'intérêts supérieurs, les relations sont bonnes et l'on observe même une certaine complémentarité à l'œuvre entre l'action de l'État et celles des mouvements religieux privés. Dans des pays où la société civile et les acteurs non étatiques restent pour le moins inexistants, les régimes éprouvent souvent le besoin de demander la garantie, la caution de l'État turc et de ses ambassades pour entamer un quelconque projet de coopération avec ces organisations turques. Le cas le plus représentatif est l'exemple de Fethullah Gülen et de son vaste réseau éducatif. Malgré les mauvaises relations que le mouvement entretient avec l'État en Turquie, les ambassades turques en Asie Centrale ont toujours apporté leurs soutiens aux disciples de Gülen qui ont ainsi pu plus facilement asseoir un vaste réseau d'écoles privées appuyées par des entreprises turques, elles-mêmes gérées par des expatriés turcs aux motivations religieuses affirmées.

L'implantation des mouvements islamistes turcs en Asie centrale par le biais des migrants pose la question du caractère provisoire ou définitif de cette implantation, des idées et des hommes. La plupart des migrants turcs en Asie centrale resteront sans doute dans ces pays d'accueil, car on voit déjà se multiplier les accessions à la propriété, mais surtout les mariages mixtes qui sont le meilleur indicateur du caractère définitif d'une immigration. Pour ce qui est de la prégnance des idées religieuses importées de Turquie, il convient d'être plus nuancé. En effet, leur enracinement en Asie centrale ne pourrait se faire sans concession aux pratiques et coutumes locales. Ainsi, une sorte de syncrétisme musulman turc et centrasiatique, très proches au demeurant, s'élabore chaque jour, au fur et à mesure que le temps passe et que les immigrés anatoliens prennent leurs marques dans les pays d'origine de leurs illustres aïeuls.

Quant au rêve de grandeur de la Turquie et son relent d'empire face à l'immigration turque en Asie centrale, il s'agit là d'une question complexe, dont la réponse ne peut être que partielle et temporaire. La présence des entreprises, écoles, mouvements religieux, mais aussi simples travailleurs turcs en Asie centrale a incontestablement accompagné et même aidé la Turquie à asseoir une certaine influence en Asie centrale. Toutefois cet apport ne doit pas être exagéré, car en réalité après avoir beaucoup misé sur l'Asie centrale et les retrouvailles avec les frères turcs, la Turquie est revenue à une politique plus réaliste, recentrée sur les vrais enjeux géopolitiques et stratégiques extérieurs, parmi lesquels l'Asie centrale ne figure pas. L'aventure centrasiatique, pétrie des illusions de retour dans la patrie originelle et mythique des Turcs, aura fait long feu et démontre que 
la Turquie regarde droit devant elle, vers l'Europe, et se détourne de son passé asiatique. Farouchement euro-centrée, c'est sur les millions d'immigrés turcs citoyens de l'Union européenne que la Turquie semble s'appuyer pour forcer la forteresse européenne où se concentrent ses intérêts les plus vitaux.

\section{Références bibliographiques}

ATEŞ Toktamış, KARAKAŞ Eser, ORTAYLI İlber (Dir.), Barış Köprüleri. Dünyaya Açılan Türk Okullar [Les ponts de la paix. Les écoles turques ouvertes sur le monde], Istanbul, DA (Ufuk Kitap 60, Güncel Kitaplar Dizisi 8).

BALCI Bayram (1999) Turcs et Turkmènes : les retrouvailles. Carnet d'une mission au Turkménistan, Revue Française de Géoéconomie, 10, pp. 165-175.

BALCI Bayram (2001) Hoca Ahmet Yesevi : politiques identitaires de la Turquie autour d'un cheikh centrasiatique du XIe siècle, Asien Afrika Lateinamerika, 29, pp. 91-101.

BALCI Bayram (2003a) Missionnaires de l'islam en Asie Centrale. Les écoles turques de Fethullah Gülen, Paris, Maisonneuve \& Larose, Istanbul, IFEA (Passé ottoman, présent turc).

Bayram BALCI (2003b) Les Ouzbeks d'Arabie saoudite entre intégration et renouveau identitaire via le pèlerinage, Central Asian Survey, 22 (1), pp. 23-44.

BALCI Bayram (2005) Les relations entre la Turquie et l'Asie centrale turcophone, 1991-2004, Outre-Terre, Revue Française de Géopolitique (Turquie-Europe Express, 2014 ? [La question de Chypre]), 10, pp. 297-316.

BALCI Bayram (2003c) Fethullah Gülen's missionnary schools in Central Asia: education, proselytism and Turkish Foreign Policy, Religion, State and Society, Oxford, June 03, pp. 151-177.

BALCI Bayram (2003d) Fethullah Gülen's Missionary Schools in Central Asia and their Role in the Sprading of Turkism and Islam, Religion, State \& Society, 31 (2), pp. 151-177.

BALCI Bayram (2004a) Between Sunnism and Shiism: Islam in post-Soviet Azerbaijan, Central Asian Survey, 23 (2), pp. 205-217.

BALCI Bayram (2004b) Islam et Éducation islamique en Azerbaïdjan indépendant. Premiers résultats d'une recherche en cours, Religioscope, 18 p., http://religion.info/french/articles/ article 54.shtml.

BALCI Bayram (2008) Education, Nationalism, and Hidden Da'wa: Turkish Missionary Movements in Central Asia and the Caucasus, in Proselytization Revisited: Right Talks, Free Markets and Culture Wars, London, Equinox, pp. 365-387.

BALCI Bayram et BUCHWALTER Bertrand (collab.) (2001) La Turquie en Asie Centrale. La conversion au réalisme (1991-2000), Istanbul, IFEA, les Dossiers de l'IFEA (La Turquie d'aujourd'hui), 5.

BALCI Bayram et MOTIKA Raoul (Éds.) (2007) Religion et politique dans le Caucase postsoviétique (Les traditions réinventées à l'épreuve des influences extérieures), Paris, Maisonneuve \& Larose, Istanbul, IFEA.

BAZIN Louis (1986) Les peuples turcophones d'Eurasie : un cas majeur d'expansion linguistique, Hérodote (spécial Géopolitique des Langues), 46, pp. 75-109.

BEZANIS Lowell (1994) Soviet Muslim Emigres in the Republic of Turkey, Central Asian Survey, 13 (1), pp. 59-180.

BOZAY Kemal (2005) “... Ich bin stolz, Turke zu sein!”. Ethnisierung gesellschaftlicher Konflikte im Zeichen der Globalisierung, Schwalbach.

COPEAUX Étienne (1992) Les «Turcs de l'extérieur » dans Türkiye : un aspect du discours nationaliste turc, CEMOTI, 14, pp. 31-52.

COPEAUX Étienne (1992) Le rêve du Loup Gris, Hérodote, 64, pp. 183-194. 
COPEAUX Étienne (1994) Manuels scolaires et géographie historique : le cas turc, Hérodote, 74-75, pp. 196-240.

COPEAUX Étienne (1997) Espace et temps de la nation turque. Analyse d'une historiographie nationaliste. 1931-1993, Paris, CNRS Éditions.

COPEAUX Étienne (1997) Les territoires de référence des discours identitaires turcs, in J. Bonnemaison, L. Cambrézy et L. Quinty-Bourgeois Dir., Le territoire, lien ou frontière ? Identités, conflits ethniques, enjeux et recompositions territoriales. Actes du Colloque de la Sorbonne (2-4.10.1995), Bondy, Éditions de l'ORSTOM (Colloques et CD ROM), 9 p.

COPEAUX Étienne (2000) Une vision turque du monde à travers les cartes de 1931 à nos jours, Paris, CNRS Éditions.

ÇAĞATAY Ergun (Photographies), KUBAN Doğan (Textes) (2006) The Turkic Speaking Peoples: 2000 Years of Art and Culture from Inner Asia to the Balkans, Munich, Prestel Verlag \& The Hague, Prince Claus Fund Library.

DEMIR Emre (2007) The Emergence of a Neo-Communitarian Movement in the Turkish Diaspora in Europe: Settlement and Competition Strategies of the Gülen Movement in France and in Germany, in Muslim Words in Transition: Contribution of the Gulen Movement, London, House of Lords, SOAS, London School of Economics, http://www.gulenconference.net/index.php/ library/articles.

DİYİH (Dış İlişkiler ve Yurtdışı İşçiler Hizmetleri Genel Müdürlüğü) (2000-2010), Yllı Raporu. Yurtdışındaki Vatandaşlarımıza İlişkin Gelişmeler ve Sayısal Bilgiler, Ankara, TC Çalışma ve Sosyal Güvenlik Bakanlığı, Annuel [voir en particulier les dernières livraisons des années 2005 et 2007 parues en 2006 et 2010 qui intègrent des chapitres Azerbaïdjan, Kazakhstan et pour la dernière Ouzbékistan].

FENOT Anne et GINTRAC Cécile (2005) Achgabad, une capitale ostentatoire. Urbanisme et autoritarisme au Turkménistan, Paris, L'Harmattan-IFEAC.

GÜLER Müjdat (2007) Orta Asya ve Kafkaslara Türk Bakışı [Vision turque de l'Asie centrale et du Caucase], Istanbul, İlgi Kültür Sanat Yay. (İnceleme Araştırma), 34 (14).

JOHANSON Lars (2006) The Borders of Turcia: Connections and Divisions in the Development of the Turkic Peoples, in E. Cagatay and D. Kuban Eds., The Turkic Speaking Peoples: 2000 Years of Art and Culture from Inner Asia to the Balkans, Munich, Prestel Verlag \& The Hague, Prince Claus Fund Library, pp. 18-29.

KORKUT Şenol (2010) The Diyanet of Turkey and Its Activities in Eurasia after the Cold War, Acta Slavica Iaponica, Hokkaido University International Symposium (The South Ossetian Conflict and Trans-border Politics in the Black Sea Rim), Tome 28, pp. 117-139.

LANDAU Jacob M. (1981), Pan-Turkism in Turkey. A Study in Irredentism, London, C. Hurst \& Co.

LANDAU Jacob M. (1995a) Pan Turkism: From Irredentism to Cooperation, Indiana University Press.

LANDAU Jacob M. (1995b) Ethnonationalism and Pan-nationalism in Turkey and the Ex-Soviet Republics, Migration, 28, pp. 67-84.

LARUELLE Marlène et PEYROUSE Sébastien (2006) Asie centrale, la dérive autoritaire (Cinq républiques entre héritage soviétique, dictature et islam), Autrement (CERI).

PAHLAVI Pierre Cyril Teymour (2002) La diplomatie culturelle à l'ère de l'interdépendance globale. La Turquie à la recherche des éléments fédérateurs de l'identité panturque, Études Internationales, 33 (2), pp. 247-274, http://www.diploweb.com/asie/pahlavil.htm.

PARLAK Nukrettin (2007) Orta Asya-Kafkasya-Balkan Ülkeleriyle İlişkiler ve Türk Dış Yardımları (1992-2003) [Les Relations et les Aides extérieures turques avec les pays d'Asie Centrale, du Caucase et des Balkans (1992-2003)], Ankara, TIKA, 91.

POUJOL Catherine (2005) Ouzbékistan. La croisée des chemins, Paris, Belin-La Documentation Française (Asie Plurielle).

SALK Gundula (1996) Die türksprachigen Afghanistanflüchtlinge in der Türkei, Materiala Turcica, 17, pp. 67-76. 
SVANBERG Ingvar (1989a) Kazak Refugees in Turkey. A Study of Cultural Persistence and Social Change, Acta Universitatis Upsaliensis, 8.

SVANBERG Ingvar (1989b) Turkistani Refugees, in P.-A. Andrews Dir., Ethnic Groups in the Republic of Turkey, Dr. Ludwig Reichert Verlag, Wiesbaden, pp. 591-601.

TAPIA Stéphane de (1998) Les réfugiés dans la construction de l'État-nation turc, Autrepart, 7, pp. 5-21.

TAPIA Stéphane de (2008) Le nouvel oléoduc Bakou-Tbilissi-Ceyhan : quels impacts pour la Turquie et l'Union européenne ?, Café géographique, in La planète en mal d'énergies ; $18^{\text {ème }}$ Festival International de Géographie de Saint-Dié des Vosges, 2007, http://www.cafe-geo.net/ article.php3?id_article=1229 ; http://fig-st-die.education.fr/actes/actes_2007/de_tapia/article. pdf.

TÜRK Fahri (2009) Azerbaycan ve Orta Asya'da Değişim Sürecinde Ortaya Çıkan Turancı Siyasi Hareketler (1989-2007) [Les mouvements pantouraniens apparus dans la période de transition en Azerbaïdjan et en Asie centrale (1989-2007)], Bilig, Türk Dünyası Sosyal Bilimler Dergisi, 51, pp. 205-230.

YAZGAN Turan (2003) Türkiye'nin Türk Dünyasındaki Eğitim-Öğretim Faaliyetleri [Les activités éducatives de la Turquie dans le Monde turc], Кати-IS, 8 (2), pp. 2-21.

YUNUSOV Arif (2009) Migration Processes in Azerbaijan, Bakou, Institute of Peace and Demography, Adiloğlu. 


\title{
Mouvements migratoires entre la Turquie et les Républiques turcophones du Caucase et d'Asie centrale : les impacts religieux
}

\author{
Bayram BALCI et Stéphane de TAPIA
}

Turquie, Azerbaïdjan et nouveaux pays indépendants d'Asie centrale partagent nombre de traits culturels, à commencer par l'appartenance au même groupe linguistique et, majoritairement, à l'islam Sunnite (ou Chiite dans le cas azerbaïdjanais). Cependant leurs histoires politiques ont largement divergé, et ce bien avant la mainmise russe puis soviétique sur les vastes territoires d'Asie centrale. Le retour à l'indépendance a autorisé de grands espoirs en Turquie, espoirs relevant aussi bien de la géopolitique que de l'économie et de la sphère linguistique et culturelle. De très nombreux accords bilatéraux ont été signés et l'Asie centrale turcophone a attiré de nombreux migrants turcs pour des raisons diversifiées. La présente contribution s'attache à l'examen d'un aspect moins connu, mais tout aussi important, celui de l'investissement religieux turc dans les territoires nouvellement indépendants, avec ses succès et ses déceptions, le tout sur fond de laïcité partagée théoriquement par la Turquie laïque kémaliste et les républiques centrasiatiques, soviétiques il y a encore peu de temps.

\section{Migrations between Turkey and Turkic-speaking Republics in Caucasus and Central Asia: Religious Impacts}

\author{
Bayram BALCI and Stéphane de TAPIA
}

Turkey, Azerbaijan and new independent countries of Central Asia share a lot of cultural elements, as belonging to the same linguistic family and, for a great majority, to Sunnî Islam, or in the case of Azerbaijan, to Shiî Islam. Nevertheless, their political histories have broadly diverged, since a long time, even before Russian and Soviet eras and political control on their territories. Return to political independence and sovereignty has authorised very great hopes and expectancies in Turkey, in various domains as geopolitics, economics, linguistic and cultural topics. A very great amount of bilateral agreements have been signed and Turkic speaking Central Asia has drained numerous Turkish migrants for diversified reasons. The present paper wants to examine a less studied, but very important, aspect which are those of the Turkish religious investments in these newly independent countries, with their successes and deceptions, even if Kemalist and secularist Turkey officially shares the same ideal of secularism with all these Central Asian republics which were members of Soviet Union, a few time ago. 


\title{
Movimientos migratorios entre Turquía y las Repúblicas de lengua turca en el Cáucaso y en Asia central: los impactos religiosos
}

\author{
Bayram BALCI y Stéphane de TAPIA
}

Turquía, Azerbaiyán y nuevos países independientes de Asia central comparten numerosos rasgos culturales, empezando por su pertenencia al mismo grupo lingüístico y, en su mayor parte, al islam Sunnita (o Chiita en el caso azerbaiyano). Sus historias políticas, sin embargo, han discrepado mucho, aun antes el dominio ruso y luego soviético en los amplios terrenos de Asia central. La vuelta a la independencia ha generado grandes esperanzas en Turquía tanto con respecto a la geopolítica como a la economía y a la esfera lingüística y cultural. Se han firmado numerosos acuerdos bilaterales y el Asia central de lengua turca ha atraído un número importante de migrantes por diversas razones. La presente contribución se dedica al examen de un aspecto menos conocido aunque igualmente importante: la implicación religiosa turca en los territorios recién independientes, con sus éxitos y sus desilusiones, teniendo en cuenta la laicidad teóricamente compartida por la Turquía laica kemalista y las repúblicas centroasiáticas que solo se deshicieron del sovietismo hace poco. 


\section{Note de recherche}

\section{La diaspora kazakhe et la politique de rapatriement de la République du Kazakhstan}

\section{Gulnara MENDIKULOVA ${ }^{1}$}

ujourd'hui les processus inter et intra-ethniques, dus à d'importants bouleversements mondiaux socio-économiques, politiques et culturels, ne cessent de s'intensifier et ils n'épargnent pas le Kazakhstan. Ce pays indépendant et multiethnique apparaît sur la scène internationale comme un État moderne et démocratique grâce à la consolidation et à l'unification de la nation, incluant les populations kazakhes qui vivent à l'extérieur des frontières nationales. Près de 4,5 millions de Kazakhs vivent disséminés à l'étranger, dans les quatorze autres États issus de l'éclatement de l'URSS et vingtcinq autres pays du monde ; 800000 d'entre eux constituent la diaspora et 3,7 millions constituent l'irredenta, c'est-à-dire les Kazakhs qui vivent hors des frontières dans des espaces limitrophes du Kazakhstan (principalement en Fédération de Russie, Chine et Ouzbékistan) depuis des temps plus ou moins anciens, du fait d'ambitions impériales et/ ou de jeux de pouvoir de la part du régime tsariste puis du pouvoir soviétique.

Irredenta et diaspora partagent un trait commun : chacune considère le Kazakhstan actuel comme sa patrie historique dont elle estime faire partie en qualité de ressortissant de droit. Les origines de la diaspora appartiennent à l'histoire, mais le présent s'inscrit dans le développement du Kazakhstan contemporain.

Cet article est d'autant plus d'actualité que le Kazakhstan, membre de plein droit de la communauté internationale, doit répondre à l'épineuse question des relations, tant au niveau international qu'intergouvernemental, entre trois composantes essentielles : la République du Kazakhstan, les représentants de la diaspora et de l'irredenta, et le pays d'accueil de ces dernières. Diaspora et irredenta sont des éléments très importants de la politique extérieure du Kazakhstan vis-à-vis des pays d'accueil. En tant que minorité ethnique en milieu étranger, la situation des Kazakhs pèse donc dans les relations entre États.

1 Prof. Dr., Director, Centre of Diaspora Studies, The World Association of the Kazakhs, U1. Valikhanova 117, kv. 35, 050010 Kazakhstan Almaty ; gmendikulova@hotmail.com L'article a été traduit du russe au français par Vanessa Balci. 
Les statuts que possèdent les Kazakhs dépendent de la politique d'accueil des gouvernements en place et conditionnent partiellement l'action du Kazakhstan à leur égard sur la scène internationale. Forts d'une expérience d'adaptation et parfois de survie en milieu étranger, dans un contexte nouveau d'une économie de marché, les membres de la diaspora participent au développement socio-économique de leur pays d'origine. Porteurs de savoirs traditionnels, ils sont en mesure de contribuer au renouveau culturel d'une nation kazakhe qui a connu une forte acculturation sous la période soviétique.

Nous abordons ici l'analyse des aspects théoriques et pratiques liés à la diaspora et à l'irredenta kazakhes ainsi que la politique de rapatriement du Kazakhstan.

\section{DIASPORA ET IRREDENTA KAZAKHES}

Sheffer définit la diaspora comme un « groupe appartenant à une minorité ethnique, ayant quitté sa patrie, vivant et agissant dans un pays d'accueil, tout en conservant des liens sentimentaux et matériels forts avec la patrie d'origine » (1986: 3). La diaspora kazakhe résulte de migrations sur de longues distances. D’abord temporaires, elles sont devenues définitives et passent par plusieurs frontières interétatiques : celles du Kazakhstan soviétique, de la Chine, des autres États d'Asie centrale, de l'Afghanistan et de l'Iran, et de là vers le reste du monde. À partir des années 1960, les représentants de la diaspora participent d'un premier exode rural et en quittant les campagnes pour les villes, ils changent d'orientation socio-économique et professionnelle.

Tout au long de son histoire, la diaspora fut, pour des raisons politiques et religieuses, le produit de migrations forcées et violentes et ce, jusque dans les années 1960 où commence à se développer une émigration économique vers l'Europe occidentale, l'Amérique et le golfe Arabo-persique. Jusqu'au début des années 1960, les migrations des Kazakhs se distinguaient par leur caractère collectif, aujourd'hui il s'agit davantage de déplacements individuels, suivis d'un regroupement familial dans le pays d'accueil. Selon Ashkenasi (1990), la diaspora se caractérise par des relations entre trois composantes :

- un groupe ethnique, doté de formes linguistiques, religieuses et historiques propres ;

- une patrie, c'est-à-dire un espace géographique pour lequel le groupe ethnique a un attachement ;

- le pays d'accueil, c'est-à-dire l'État où vit actuellement la diaspora.

Dans le cas de la diaspora kazakhe, on constate que ses membres représentent toutes les catégories sociales de la nation, partagent une même patrie historique, sont de confession musulmane pour la plupart et appartiennent à la famille des peuples turcophones.

Cette diaspora compte environ 800000 individus dispersés dans divers pays d'accueil. Ils ne constituent pas un groupe ethnique vivant dans un espace propre, sauf en Turquie, en Iran ou en Afghanistan où les Kazakhs ont tendance à se regrouper encouragés en cela par les politiques du pays de résidence. Considérés comme une minorité ethnique, ils n'ont jamais pesé sur les politiques locales. 
Parmi les raisons politiques qui ont entraîné les premières dispersions on peut citer les guerres kazakho-djoungares du XVIIIe siècle, les insurrections et les guerres contre l'autocratie tsariste aux XVIIIe et XIXe siècles, le mouvement de libération nationale en Asie centrale contre l'empire tsariste en 1916, l'établissement du pouvoir soviétique et la Guerre civile, le génocide des Kazakhs sous la collectivisation, la lutte contre les communistes chinois au Turkestan oriental (actuel Xinjiang) ou les effets de la Seconde Guerre mondiale. Les raisons économiques qui ont poussé à la migration tiennent à la politique agraire de Stolypine à l'époque tsariste, la destruction du mode de vie nomade traditionnel après l'intégration du Kazakhstan à la Russie et sous la collectivisation soviétique (1928-1932), l'émigration économique des descendants des réfugiés vers les pays d'Europe occidentale et d'Amérique dans les années 1960-1990, l'instabilité économique du Kazakhstan après l'effondrement du système socialiste et de l'économie de l'URSS au début des années 1990 et la transition vers l'économie de marché. Les raisons religieuses sont liées, aussi bien sous les Tsars que sous les Soviets, à l'interdiction pour les fidèles de quitter le pays et d'accomplir le pèlerinage à La Mecque et à Médine, lieux sacrés de l'Islam. Ceux qui arrivaient à partir en pèlerinage tentaient de rester dans les pays d'Orient pour se conformer aux prescriptions de l'Islam. Aujourd'hui encore, dans les villages de Şekerköy et Akhunköy en Turquie, vivent les descendants d'une trentaine de familles kazakhes qui ont accompli le hadj au XIXe siècle et sont restés sur place depuis cette époque (Mendikulova, $2006: 24$ ).

La diaspora est à la fois objet de politique intérieure et sujet des relations internationales. Ses caractéristiques correspondent aux orientations de la politique ethnique qui prend en compte les « co-ethniques » séparés de la nation (l'irredenta), l'ethnicité comme ressource stratégique, les nations sans État, les communautés ethniques transnationales et l'internationalisation des droits des minorités. À ce titre elle est d'une part un élément essentiel du pluralisme ethnique qui joue sur les relations internationales et elle exerce d'autre part une influence sur les conflits ethniques internes (Sheffer, 1986). Ainsi, Milton J. Esman (1990 : 83-88) considère que le premier levier pour un pays en difficulté est d'appeler à l'aide sa diaspora² .

Le second levier s'appuie sur des actions qui ont une influence directe sur les évènements dans le pays d'origine. Elles peuvent relever d'initiatives indépendantes ou être inspirées par le pays d'accueil ou le pays d'origine et contribuer aussi à freiner les intérêts du pays d'accueil comme ceux du pays d'origine. En général, le gouvernement du pays d'origine est plutôt favorable à une instrumentalisation de sa diaspora dans le but d'obtenir des moyens financiers ou un soutien diplomatique, mais il est plus rare qu'il accueille avec bienveillance l'ingérence de celle-ci dans la gestion des affaires intérieures. Dans le cas kazakh, ce levier est d'abord économique. Les représentants de la diaspora en Turquie, aux États-Unis et en Europe occidentale possèdent une riche expérience de l'entreprise dans le monde capitaliste. Le succès des Kazakhs en Turquie est à cet égard un bon exemple puisque la communauté réunie en corporation a permis le développement de toute la filière du cuir en Turquie, en Grande-Bretagne, en France et dans d'autres pays. Forte de ces savoir-faire, elle est disposée à organiser des filières de production au Kazakhstan.

2 Durant la Première Guerre mondiale, par exemple, l'État allemand fit appel à sa diaspora aux États-Unis pour militer contre l'entrée en guerre des Américains aux côtés de la Triple Entente. 
Le troisième levier, d'après Esman (1990), réside dans la défense des droits et intérêts de la diaspora par le pays d'origine, selon le droit international. Il concerne essentiellement la migration ouvrière. De nombreux accords et traités interétatiques concernent les conditions de travail, confirmant le droit des gouvernements à protéger leurs compatriotes. C'est le cas du rôle qu'a joué l'État dans le rapatriement des Kazakhs d'Iran et d'Afghanistan qui ont commencé à revenir dans leur pays après les accords de février 1993 entre l'Iran et le Kazakhstan.

Il existe aussi un quatrième levier qui concerne le cas de deux groupes diasporiques respectivement installés dans le pays d'origine de l'un et de l'autre. On donnera pour exemple les Allemands de la Volga installés au Kazakhstan et les Kazakhs résidant en Allemagne. La situation de ces diasporas ethniques ne va pas sans jouer un rôle sur les relations entre les deux États. Ainsi, l'Allemagne qui accueille un petit nombre de Kazakhs, 178 familles tout au plus (soit près de 900 individus), les considère avec plus de tolérance et de bienveillance qu'elle ne le fait pour les immigrés turcs. Cette attitude ne s'explique pas seulement par la faiblesse numérique des Kazakhs d'Allemagne, mais aussi par la présence de centaines de milliers d'Allemands au Kazakhstan déportés dans les années 1940 après la fin de l'autonomie des Allemands de la Volga. Et si beaucoup d'entre eux sont retournés en Allemagne, à leurs yeux, le Kazakhstan demeure leur seconde patrie. Tous ces épisodes de l'histoire du peuple allemand pèsent dans la relation qu'il a avec la diaspora kazakhe en Allemagne. C'est la raison pour laquelle ces processus d'influence réciproque, tant sur le pays d'accueil que sur le pays d'origine, sont importants pour comprendre un des aspects des politiques internationales.

En 1992, devant le premier Congrès Mondial des Kazakhs, les médias ont commencé à parler de « diaspora » pour désigner l'ensemble des Kazakhs vivant au-delà des frontières du Kazakhstan. Or, en analysant les raisons qui font que $35 \%$ de nos compatriotes (soient 4,5 millions de Kazakhs) se retrouvent en dehors des frontières de la république, on s'aperçoit que le terme de « diaspora » ne peut s'appliquer à tous les groupes dispersés dans près de quarante pays. En effet, seulement 800000 d'entre eux, soit $18 \%$ des « co-ethniques », forment la diaspora, selon le sens qu'en donne Sheffer. 3,7 millions de Kazakhs de l'étranger vivent sur des territoires limitrophes du Kazakhstan : Russie (régions d'Astrakhan, Orenbourg, Kourgan, Omsk et République autonome de l'Altaï), Ouzbékistan (districts du Syr-Daria, Tchirtchik, Kyzylkoum, Myrzashol), Chine (districts de l'Altaï, Tarbagataï, l'Ili, Kouldja, Erenkabyrg, Barköl-Kumul et Province Autonome du Xinjiang-Ouïghour), ainsi que dans l'aïmak ou province de Bayan-Ölgiy en Mongolie. Ceux-là ne peuvent être assimilés à la « diaspora », puisqu'ils vivent là où ils ont toujours vécu. Mais la politique coloniale de l'empire tsariste aux XIXe et XXe siècles, et ensuite le régime totalitaire soviétique au XXe siècle, ont fait qu'ils se retrouvent aujourd'hui en dehors des frontières de leur pays. Ce sont eux que nous nommons irredenta (Mendikulova, 1995, 1996, 1997, 2006).

À la différence des diasporas, l'irredenta est un groupe qui, à un moment donné, s'est trouvé séparé de sa patrie par des guerres, des annexions, des contestations de frontières, des découpages coloniaux (Esman, 1990 : 83-84). Cette catégorie de la population était déjà connue des historiens du siècle passé qui la définissaient comme « le produit de la doctrine proclamant le droit de chaque peuple à s'instituer comme "nation" " (Cobban, 
1969). C'est pourquoi j'ai proposé dans le cadre conceptuel des sciences sociales kazakhstanaises, le terme d'irredenta appliqué aux Kazakhs vivant sur des territoires limitrophes du Kazakhstan ${ }^{3}$.

L'irredenta kazakhe en Russie est le résultat de siècles de conquêtes territoriales et d'annexions depuis le XVIe siècle de territoires traditionnellement kazakhs - comme la conquête du khanat de Sibérie par Ermak -, ainsi que du découpage en 1924 de territoires du nord, de l'ouest et de l'est du Kazakhstan au profit de la RSFSR au moment de la délimitation des États-nations soviétiques d'Asie centrale (Mendikulova, 1995).

Les groupes kazakhs de Chine résultent quant à eux du conflit kazakho-djoungare entre les XVIe et XXe siècles ainsi que de l'expansion territoriale de l'empire russe qui ont mené à la redéfinition de la frontière sino-russe en Asie centrale. En conséquence, les Kazakhs ont perdu une partie importante de leur territoire au nord-est, à l'est, au sud-est et au sud du pays. Les populations de ces régions forment aujourd'hui l'irredenta kazakhe de Chine (Mendikulova, 2006).

Les Kazakhs d'Ouzbékistan sont le fruit de la refonte des frontières et des États en Asie centrale par le pouvoir russe en 1924 et du découpage de terres kazakhes au profit de l'Ouzbékistan dans les années 1950-1960. En effet, en 1924, lors de la formation stato-territoriale de l'ASSR du Kazakhstan et de ses voisins centrasiatiques, des populations kazakhes ayant toujours vécu dans les zones de Syr-Daria, Tachkent, Tchirtchik, Kyzylkoum et Myrzachol furent intégrées à l'Ouzbékistan. La région autonome du Karakalpakstan fut créée en 1925 et intégrée au Kazakhstan, mais en 1932, la modification territoriale et nationale de l'ASSR kazakhe entraîna la disparition de cette région au profit d'une nouvelle république autonome soviétique socialiste (ASSR) intégrée en 1936 à la RSS d'Ouzbékistan (Koblandin et Mendikulova, 2009).

\section{SPÉCIFICITÉS DE LA DIASPORA KAZAKHE}

Depuis les années 1960, les Kazakhs qui vivent à l'Ouest illustrent parfaitement le cas de la migration volontaire. Nous abordons ici les processus actuels auxquels la diaspora doit faire face : assimilation, acculturation, conservation de l'identité ethnique ou rapatriement. Deux tendances peuvent être observées : l'une relève de tentatives de conserver et de développer une identité ethnique tout en vivant en milieu étranger et l'autre dessine un net souhait de rapatriement au Kazakhstan, surtout depuis l'accès du pays à l'indépendance en 1991.

Une des caractéristiques de la communauté kazakhe à l'étranger, au moins jusqu'en 1991, réside dans l'absence de toute organisation communautaire structurée. Ceci peut s'expliquer par la faiblesse numérique des Kazakhs, mais aussi par leur engagement au sein d'organisations et d'associations turques. Ils n'ont alors pas ressenti le besoin de créer des structures propres permettant d'améliorer leur situation socio-économique,

3 Symposium international La Diaspora kazakhe : problèmes et perspectives (16-17 novembre 1995, à Türkistan), où je présentais une communication intitulée « Problèmes historiques de l'étude de la diaspora kazakhe » (Mendikulova, 1996 : 75-78). 
leur statut juridique et politique et de transmettre à la jeune génération les traditions et la culture kazakhes.

La situation évolua après la proclamation de l'indépendance du Kazakhstan qui provoqua chez tous les Kazakhs de l'étranger un sentiment de fierté d'appartenir à l'ethnie kazakhe. Depuis les années 1990 et l'apparition sur la mappemonde du Kazakhstan indépendant, le développement du pays est rapide et permet dans un futur proche d'espérer une entrée dans le club des cinquante premiers pays les plus développés. Cet événement a été le point de départ de la création d'associations dans divers pays ; ces dernières tentant de se mobiliser et de s'organiser pour mettre en œuvre des manifestations culturelles et sportives dans le but de contribuer à préserver l'identité ethnique. Ainsi dès l'été 1994, une société sportive basée à Munich organisa un championnat de football opposant cinq équipes de la diaspora : Berlin, Cologne et Munich, Paris et Vienne (Avrupa Kazak Türkleri Bülteni, 1994).

La diaspora n'est homogène, ni en termes sociaux, ni en termes économiques, culturels, juridiques ou éducatifs. Le plus souvent, elle se compose de Kazakhs originaires des trois pays d'origine où ils vivent traditionnellement : Kazakhstan, Russie, Chine ou pays d'immigration comme la Turquie. La langue étant l'un des marqueurs essentiels de l'ethnos, au cours de mes recherches sur l'identité linguistique, je suis arrivée à la conclusion que les Kazakhs de l'étranger se caractérisent par un trilinguisme voire un multilinguisme : outre le kazakh et la langue du pays d'accueil, les Kazakhs du Kazakhstan privilégient le russe, ceux du Xinjiang, le mandarin et le mongol et ceux de Turquie, le turc.

L'usage de la langue kazakhe par les deuxième et troisième générations de la diaspora vivant en Europe, aux États-Unis ou au Canada a un avenir incertain. La première génération (qui ne peut être qualifiée d' «âgée », puisque la moyenne d'âge oscille entre cinquante et cinquante-cinq ans et que ceux qui ont plus de soixante ans sont rares) connaît encore le kazakh mais déjà la génération suivante préfère utiliser au quotidien la langue du pays d'accueil. Bien que quelques jeunes aspirent à apprendre le kazakh, stimulés par le développement du pays, la langue d'origine est cependant moins usitée que d'autres langues. Inexistantes en France, aux États-Unis ou ailleurs, les écoles de langue sont le fait d'associations basées en Turquie ou en Grande-Bretagne. En outre, la question linguistique est étroitement liée au problème des trois alphabets utilisés par les Kazakhs des différents pays pour écrire leur langue. Les Kazakhs du Kazakhstan utilisent l'alphabet cyrillique, ceux de Turquie l'alphabet latin et ceux de Chine l'écriture arabe, ce qui complique considérablement la compréhension, l'approche des littératures et documents écrits, envoyés du Kazakhstan à destination des communautés kazakhes d'Europe occidentale.

En Amérique du Nord, les Kazakhs vivent majoritairement dans des familles nucléaires, car les enfants et l'épouse ont émigré dans le cadre du regroupement familial. En Turquie et dans d'autres pays du Moyen-Orient, ils préfèrent vivre dans des familles élargies : les parents vivent avec leurs enfants non mariés, mais aussi avec les fils mariés, les belles-filles et les petits-enfants. Cette situation est aussi peut-être la conséquence de la cherté du logement et de la terre, comme, par exemple, en Turquie, où il est plus économe et plus confortable de vivre ensemble ou quand les moyens manquent pour permettre au fils marié d'acquérir un logement. 
Les mariages intra-ethniques dominent dans les première et deuxième générations. Lors de nos entretiens dans différents pays, nous avons pu constater que la majorité n'accueille pas favorablement le mariage mixte. Le mariage entre turcophones est plus ou moins bien toléré, ce qui s'explique par des racines communes et laisse par conséquent présager d'une meilleure compatibilité et solidité du couple dans le mariage. Cette différenciation ethnique à propos des relations matrimoniales et familiales résulte d'une conscience de soi tendant à isoler les Kazakhs des autres ethnies quand ils sont en milieu étranger, tout autant qu'elle souligne l'appartenance à l'ethnos kazakh. En outre, les divisions tribales ont une signification particulière pour la préservation de la conscience ethnique et l'appartenance lignagère garde toute son importance en émigration. Le refus de mariages mixtes s'explique également par le non moins important facteur religieux, surtout pour les Kazakhs du Xinjiang, où l'Islam joue un rôle majeur. La religion musulmane recommande que chaque fidèle se marie avec l'un(e) de ses coreligionnaires : à défaut d'épouser un(e) musulman(e), il/elle préférera un(e) fidèle du Livre sacré, chrétien ou juif. Cependant, l'Islam recommande que les enfants nés de tels mariages, dans la mesure du possible, soient éduqués dans la foi musulmane.

Les membres des seconde et troisième générations qui ont passé leur jeunesse dans l'Europe occidentale des années 1990 ont un tout autre point de vue sur la question. Pour eux, la motivation ethno-religieuse n'est pas si importante. La tradition des mariages intra-ethniques (entre Kazakhs) laisse progressivement la place à des mariages interethniques et ce, pour différentes raisons : l'obtention de la nationalité du pays d'accueil, ou le choix de l'amour plutôt que celui de la raison. Le nombre des mariages mixtes est particulièrement important parmi les Kazakhs de France, mais, d'après le témoignage d'un représentant local de la diaspora, beaucoup de Kazakhs (venus de Turquie pour la plupart) se marient avec des Françaises, certains dans le but d'obtenir le statut de résident permanent. L'analyse des mariages interethniques chez les Kazakhs de la diaspora montre qu'avant tout ils concernent les hommes, arrivés seuls, sans famille, en Europe occidentale pour des raisons professionnelles ou économiques, pour des études ou un travail. La part des mariages mixtes impliquant une jeune fille augmente progressivement, mais il s'agit pour la plupart de jeunes filles du Kazakhstan et non de la diaspora, ce qui montre encore la réticence des vieilles générations à permettre de telles unions, même s’ils commencent à tolérer celles de jeunes hommes.

La diaspora kazakhe tente de préserver son identité ethnique, grâce aux liens étroits qu'elle entretient avec la mère-patrie historique et nombreux sont ses membres à vouloir visiter et connaître le pays pour y retrouver des parents et leurs racines. On observe aussi une tendance nouvelle au « retour » définitif au Kazakhstan.

\section{LA POLITIQUE DE LA RÉPUBLIQUE DU KAZAKHSTAN VIS-À-VIS DE LA DIASPORA}

Ce n'est qu'à l'indépendance du pays qu'il fut donné à la société kazakhstanaise d'étudier l'histoire et la situation actuelle des Kazakhs vivant dans les différents pays étrangers et d'établir des liens étroits avec eux. La politique de l'État vis-à-vis de la diaspora est liée au développement d'un Kazakhstan multiethnique, attentif non seulement 
à la consolidation et à l'unité du peuple kazakhstanais, comme communauté nouvelle d'individus vivant dans les frontières de la République, mais aussi à la défense de compatriotes que le destin a placés au-delà des frontières de la patrie historique.

À cet égard, l'Association Mondiale des Kazakhs joue un rôle majeur pour les communautés de l'étranger et se trouve au centre de la politique gouvernementale envers la diaspora et les irredenta. Après la proclamation de l'indépendance, il fut décidé de réunir au Kazakhstan les représentants de ces populations pour coordonner les actions de l'État en leur faveur. Le 29 septembre 1992 à Almaty, dans les salons du théâtre Abaï eut lieu le premier Congrès Mondial des Kazakhs auquel participèrent les représentants des Kazakhs vivant en Turquie, Allemagne, France, Suède, Norvège, Mongolie, Russie, Kirghizstan, Ouzbékistan et Turkménistan. Résolution fut prise d'œuvrer pour intensifier les relations entre le Kazakhstan et les Kazakhs de l'étranger et ce, dans tous les domaines. Seuls manquaient les Kazakhs de Chine que les autorités de Pékin n'avaient pas autorisés à se rendre à la manifestation. Ce jour-là, le gouvernement se posa en protecteur de tous les Kazakhs de par le monde. Cette initiative permit pour la première fois aux représentants de la diaspora et de l'irredenta, qui n'avaient pas eu l'occasion de fouler la terre de leurs ancêtres depuis des décennies, de se rencontrer et de connaître leurs compatriotes.

Le congrès entérina la création d'une nouvelle organisation pour coordonner les actions de toutes les communautés kazakhes expatriées, mais aussi pour étudier les spécificités historiques, ethnographiques, culturelles et linguistiques de la diaspora. C'est pourquoi, le 17 et 18 mars 1993, lors de la cinquième conférence de la société Kazakhstan fondée en 1976, on reconnut l'importance de donner aux Kazakhs de l'étranger l'opportunité de se familiariser avec leur histoire, leur culture d'origine et les avancées scientifiques et économiques du pays. Cette société devait ainsi apporter son aide aux expatriés pour soutenir les relations culturelles avec le pays. Le président de la République du Kazakhstan, Nursultan A. Nazarbaev fut élu président du bureau de l'Association Mondiale des Kazakhs et Kaldarbek Naïmanbaev en fut élu secrétaire général. La société Kazakhstan fut intégrée par décret à la nouvelle structure. Devenue le successeur légal de Kazakhstan, 1'Association hérita alors de son patrimoine et de sa documentation.

Le 14 février 1994 s'ouvrit à Almaty un centre d'affaires rattaché à l'Association et financé par des hommes d'affaires kazakhs de Turquie, de Russie et d'ailleurs, dans un objectif de développement commun. Elle prévoyait aussi l'ouverture d'un centre de presse et d'un centre culturel. En outre, il fut prévu, lors d'une session tenue à Almaty en mars 1994, d'attribuer, sur critères d'excellence, une vingtaine de bourses à des étudiants venant étudier au Kazakhstan.

À l'été 1994, l'Association conclut un accord avec des représentants du ministère de l'Instruction et des Établissements d'enseignement supérieur, invitant quarante Kazakhs de l'étranger à venir poursuivre leurs études au Kazakhstan. Grâce à des bourses, ces étudiants bénéficièrent d'une année d'études à Karatau (région de Djambul). L'Association obtint que le ministère aide ceux qui font le choix d'étudier au Kazalhstan ou le souhaitent. Dès l'année universitaire 1995-1996, 150 étudiants (dont des Kazakhs de Chine, Mongolie, Iran et Russie) bénéficièrent ainsi d'une aide pour suivre l'année préparatoire de l'Institut d'Agronomie d'Almaty, de l'Institut pédagogique d'État Abay, de l'Université 
d'État Abylay Khan ou des Départements de Langues des Facultés de Djambul, Türkistan et Taldykurgan (Deuletov, 2002 : 10). De 1995 à 1998, plus de 300 étudiants ont réussi l'année préparatoire et ont pu intégrer les facultés de leur spécialité. En octobre 1994, sous la direction du secrétaire général de l'Association, une délégation se rendit en Turquie, à Istanbul et Salihli, pour célébrer le grand poète kazakh Abaï, dont le Kazakhstan fêtait l'anniversaire sous l'égide de l'UNESCO. Les médias turcs ont souligné à l'époque l'importance de la manifestation pour la consolidation des relations entre les deux peuples. En mars 1995, à Istanbul se tint un Congrès des Kazakhs des pays de la région (Turquie, Kazakhstan, Ouzbékistan, Russie, Afghanistan et Iran) qui procéda au bilan de l'action de l'Association. Au cours des années 1990, plusieurs petits congrès préparatoires au Second Congrès Mondial des Kazakhs se tinrent en Russie, Chine, Mongolie et Ouzbékistan.

En 1995-1996, l'État adopta un programme d'aide à la diaspora. Ce programme visait le développement et la consolidation des relations de l'État avec la diaspora et l'irredenta. En janvier 1998, il fut question de créer un centre culturel de la diaspora en Europe, impliquant des représentants de tous les pays européens, dont la France. Ce centre, aujourd'hui basé à Cologne en Allemagne, compte quinze membres élus.

En 2001, l'ambassadeur de la République du Kazakhstan en France, en compagnie du maire de Nogent-sur-Marne, posait, le jour du dixième anniversaire de l'indépendance du Kazakhstan, une plaque commémorative devant la maison où vécut Mustafa Tchokaï. Le Premier ministre du Kazakhstan et des vétérans de la Seconde Guerre mondiale ainsi que des représentants de la diaspora kazakhe en France étaient présents à la cérémonie. Cette visite officielle fut également l'occasion d'obtenir pour les Archives Nationales du Kazakhstan des copies du Fonds Mustafa Tchokaï conservé à l'INALCO4 .

Le 20 juillet 2002, le Premier ministre signa un décret officialisant la future tenue du Second Congrès Mondial des Kazakhs qui se déroula les 23 et 24 octobre 2002 (Deouletov, 2003). D'après l'Association, il réunit plus de 400 participants venus de trente-quatre pays différents. La délégation la plus importante venait de l'Ouzbékistan voisin. Pour la première fois étaient présents des représentants des Kazakhs de Chine. Le président de la République, dans son discours inaugural, rappela les grandes orientations et priorités de la politique intérieure et extérieure du Kazakhstan et son aspiration à rencontrer les Kazakhs vivant à l'étranger lors de ses visites officielles. Le Congrès élabora un nouveau programme gouvernemental pour l'assistance aux expatriés, examinant également la question du rapatriement pour ceux qui souhaitaient revenir dans leur pays d'origine. À l'issue du Congrès, 800 familles kazakhes de Mongolie avaient déjà exprimé ce souhait. En 2004-2005, l’État dut établir un quota maximal de 15000 rapatriés, tant la demande était importante : près de 3 millions de Kazakhs expatriés disent envisager leur retour définitif.

Selon les données du Comité des Migrations auprès du ministère du Travail et de la Protection sociale, en dix ans, près de 600000 Kazakhs ethniques sont revenus au pays en bénéficiant du dispositif de retour par quota officiel, mais des sources officieuses

4 Mustafa Tchokaï (1890-1941), intellectuel turkestanais d'origine kazakhe, éphémère président des États autonomes de Kokand et d'Alash Orda, réfugié en France, a longtemps animé la résistance turkestanaise comme l'URSS. Il a vécu une grande part de sa vie à Nogent-sur-Marne [Ndlr]. 
avancent le chiffre de 1500000 rapatriés. Pour les seules années 1991-1992, où pourtant la situation économique du pays était critique, le nombre des oralman (en kazakh les personnes optant pour un retour volontaire ${ }^{5}$ ) dépassait les 60000 individus.

C'est le 18 mai 2005 que l'Association créa le Centre pour la Jeunesse étudiante, auprès de l'Association Mondiale des Kazakhs à la suite de rencontres entre spécialistes portant notamment sur la création d'un Centre de recherche consacré à l'étude des diasporas au sein de l'Association.

L'année 2005 a été marquée par la tenue à Astana, nouvelle capitale du pays, du troisième Congrès Mondial des Kazakhs organisé par un décret gouvernemental signé par le Premier ministre 6 . Il a réuni 300 délégués de trente-deux pays (Russie, Chine, Autriche, Norvège, Tchéquie, Italie, Singapour, Égypte, etc.), 200 représentants de toutes les provinces du pays et des villes d'Almaty et Astana, ainsi que les médias ${ }^{7}$. L'image retenue a été celle des délégués plantant des jeunes pousses d'arbres le long de l'avenue Atameken ${ }^{8}$. Lors de ce congrès, quatre ateliers, organisés par les représentants de différents ministères, ont été consacrés au développement de la diaspora et de la culture kazakhe, aux études supérieures pour les étudiants de la diaspora au Kazakhstan, au placement des investissements de la diaspora et à la politique migratoire sur la question des oralman. Des demandes d'enseignants spécialisés ont été formulées concernant les enseignements en langue kazakhe avec la mise à disposition de manuels et de matériel pédagogique. D'autres débats eurent lieu sur l'octroi de la citoyenneté kazakhstanaise aux oralman, sur la détermination de leur statut et sur l'assistance et les services aux rapatriés afin de leur garantir un logement ou un terrain ainsi qu'une insertion professionnelle. Les dernières questions abordées portaient sur les opportunités d'investissements au pays d'origine et sur les contributions des entrepreneurs kazakhs de l'étranger dans le développement des PME.

5 Voir la contribution de Gaëlle Lacaze dans ce même numéro.

6 Le vice-Premier ministre, A.S. Essimov, fut nommé président de la commission, avec comme adjoints : E.M. Kossoubaev, ministre de la Culture, de l'Information et des Sports, T.A. Mamashev, secrétaire général du Bureau de l'Association Mondiale des Kazakhs, O.E. Shoukeev, maire de la ville d'Astana, D.A. Kaletaev, chef du Département de Politique sociale au secrétariat de la présidence de la république. B.J. Omarov, président de la Commission pour les Langues, fut nommé secrétaire de la commission, montrant ainsi l'intérêt très direct du pouvoir central pour cette question.

7 Beaucoup de jeunes étaient présents, qu'ils soient hommes d'affaires, scientifiques, enseignants, médecins, étudiants, artistes, c'est-à-dire les personnes socialement, économiquement et culturellement mobiles parmi les actifs de ces communautés kazakhes de l'étranger. Parmi les délégués, on comptait vingt scientifiques, cinq membres du Parlement, un haut fonctionnaire, huit fonctionnaires de province, trente-neuf présidents de centres culturels kazakhs à l'étranger.

8 Avenue « de la Patrie » au centre de la nouvelle capitale, construite à partir de la ville provinciale d'Akmolinsk, rebaptisée Astana. Le nouvel état construit là une capitale moderne sur deux principes destinés à affermir son autorité : vitrine d'un Kazakhstan entreprenant, moderne, puissant, et recentrage vers le nord d'un territoire jusqu'alors déséquilibré ; le nord où se situe Astana étant bien plus russifié que le sud. De plus, Almaty est proche de la frontière kirghize et non loin de la Chine. Cette opération d'urbanisme volontaire peut être comparée au transfert de la capitale turque d'Istanbul à Ankara ou à la construction de Brasilia ou Canberra [Ndlr]. 
Lors de la séance inaugurale dans les nouveaux bâtiments de la Maison du Gouvernement, le président du Kazakhstan et le président du bureau de l'Association Mondiale des Kazakhs avaient mis l'accent sur le développement du pays et sur les communautés kazakhes de l'étranger considérés comme central dans la politique nationale. On peut donc noter une nette amélioration des relations et des échanges entre le pays et la diaspora ces dernières années, même si des problèmes subsistent quant à la garantie des intérêts de la diaspora liés au droit, à la culture, à l'information et enfin à l'éducation. La visite par les délégués de la nouvelle résidence du chef de l'État, $A k$ Orda ${ }^{9}$, motif de fierté pour les Kazakhs, a été un événement marquant, car les petits et de grands congrès mondiaux ne sont pas de simples festivités, mais d'importantes manifestations politiques pour la consolidation de la société kazakhstanaise, moments emblématiques de la construction de l'État-nation.

Le décret présidentiel de 2005 (Programme officiel d'assistance aux compatriotes, vivant à l'étranger pour 2005-2007) marque une étape importante dans le développement des relations entre l'État kazakh et la diaspora kazakhe. L'objectif de ce Programme est de "créer un système étatique de défense et de soutien juridique, économique et social aux compatriotes kazakhs, vivant à l'étranger, mais demeurant une partie indivisible de la nation kazakhe ». Pour y parvenir, le président de la République propose de mettre en œuvre plusieurs chantiers : le perfectionnement des normes juridiques concernant le soutien aux compatriotes, vivant à l'étranger ; l'établissement de liens économiques solides ; le soutien de relations culturelles et sociales de solidarité stables ; l'information des compatriotes ; la facilitation des études au sein d'établissements supérieurs au Kazakhstan ; 1'assistance à la consolidation de la diaspora kazakhe.

Pour 2011, l'Association Mondiale des Kazakhs a proposé une série de manifestations et d'actions à long terme consacrées à la consolidation de la diaspora et de l'irredenta kazakhes dans les domaines de la science, de l'éducation, de la culture, de la spiritualité, du développement économique et social et de l'essor des centres culturels. Ces actions visent toutes à préserver l'identité ethnique des Kazakhs de l'étranger et à favoriser l'étude de la langue maternelle, de l'histoire du Kazakhstan, des traditions et coutumes nationales.

9 Le nom du palais présidentiel, Ak Orda (Horde Blanche), est polysémique, il renvoie à la vie nomade des origines, aux états nomades impériaux dont le plus prestigieux fut celui de Gengis Khan. Se reporter aux références historiques médiévales comme la Horde d'Or, la Horde Bleue et aux dénominations - erronées d'ailleurs - comme celles des confédérations kazakhes d'avant l'arrivée des Russes dans la steppe : Grande, Petite et Moyenne Hordes. Si ce n'est que le kazakh emploie jüz (centurie) et non orda (armée, camp du khan, par extension état). Cf. le turc ordu (armée) [Ndlr]. 


\section{POLITIQUE DE RAPATRIEMENT DE LA RÉPUBLIQUE DU KAZAKHSTAN}

Le rapatriement des Kazakhs ethniques ${ }^{10}$ a commencé dès 1992. Deux vagues de retour peuvent être distinguées : la première va de 1992 à 1997, date de l'entrée en vigueur de la loi sur les migrations de 1997 et la seconde de 1997 à nos jours, date à laquelle les oralman peuvent se réinstaller au Kazakhstan (Mendikulova, 2004 : 3-35). Dans les années 1990, la majeure partie des rapatriés venait de Russie, de Mongolie, de Turquie, du Tadjikistan, d'Iran et d'Afghanistan (Diener, 2009). Depuis les années 2000, les retours concernent surtout des Kazakhs d'Ouzbékistan et du Turkménistan et depuis 2003, de Chine.

La diaspora kazakhe en Iran se compose de descendants d'émigrés ayant quitté le pays pendant la Guerre civile de 1918-1920 ou pendant la collectivisation de 19291932. Parmi eux figurent des Kazakhs d'Afghanistan, réfugiés en Iran depuis les années 1980. Ces derniers, considérés comme des réfugiés de guerre, ont vécu jusqu'en 1993 sans papiers et sans aucune assistance de la part du gouvernement iranien, raison pour laquelle le Kazakhstan négocia avec les autorités iraniennes leur rapatriement. En 1993, plusieurs milliers de Kazakhs d'Iran furent donc autorisés à se réinstaller au Kazakhstan. En 1996, une Loi sur l'Immigration instituait le statut de "réfugié » pour tout Kazakh ethnique rapatrié. Selon l'article 17, "le statut de réfugié est attribué à toute personne de la diaspora vivant à l'étranger ayant des liens avec la patrie historique, mais obligée de revenir au Kazakhstan car menacée d'oppression, de poursuites, de restrictions de ses droits et libertés ». Le gouvernement créa alors un département des Migrations auprès du ministère du Travail et de la Protection sociale chargé de l'organisation de ces retours. La Loi de 1997 sur les migrations stipule que chaque candidat au rapatriement reçoit le statut d'oralman. De 1993 à 2009, plus de 700000 personnes sont ainsi retournées au Kazakhstan (Statistical Yearbook of Kazakhstan, 2007 : 26-28 ; IA Novosti-Kazakhstan, 18/01/2010 ; Mendikulova, 2009). En 1998, le gouvernement adopta un Code de rapatriement des Kazakhs ethniques dans leur mère-patrie historique. L'objectif principal de ce Code est de mettre en place des procédures de rapatriement pour les Kazakhs ethniques en aidant à leur réinstallation et à leur intégration professionnelle et sociale.

Il existe au Kazakhstan une procédure d'acquisition du statut d'oralman. L'article 14 de la Loi sur La migration de la population stipule que «les personnes souhaitant obtenir ce statut doivent transmettre leur requête en mains propres à un membre majeur de leur famille, à un représentant compétent du corps diplomatique ou consulaire de la République du Kazakhstan à l'étranger ou encore à l'Agence des Migrations et de la Démographie ». La loi reconnaît comme « parent », les enfants (y compris adoptés), les époux ou les épouses, la fratrie, les ascendants et leur famille ainsi que la descendance

10 La politique de rapatriement des Kazakhs ethniques est régie par les Lois républicaines sur $L a$ citoyenneté dans la république du Kazakhstan, De l'immigration (26 juin 1992), De la migration de population (13 décembre 1997) et par le décret du cabinet des ministres De la procédure et conditions de réinstallation dans la RSS du Kazakhstan d'individus de nationalité kazakhe, qui avaient exprimé le souhait de travailler dans les zones rurales d'autres républiques et à l'étranger. 
ayant atteint la majorité. Chaque année, le quota d'immigration permet à 5000 familles, soit près de 15000 individus de bénéficier de ce statut. En 2010, le quota annuel a été revu à la hausse pour permettre l'accueil de 20000 nouveaux oralman.

\section{Nombre d'Oralman revenus au Kazakhstan, par régions (Période de 1991 au $1^{\text {er }}$ octobre 2008)}

\begin{tabular}{|c|l|c|c|c|c|c|c|}
\hline \multirow{2}{*}{$\mathrm{N}^{\circ}$} & \multirow{2}{*}{ Région } & \multicolumn{3}{|c|}{ Total } & \multicolumn{4}{c|}{ Répartition par sexe } \\
\cline { 2 - 8 } & Familles & Personnes & Hommes & $\%$ & Femmes & $\%$ \\
\hline 1 & Akmola & 7451 & 38233 & 17674 & 46,2 & 20559 & 53,8 \\
\hline 2 & Aktyube & 6819 & 33759 & 15584 & 46,2 & 18175 & 53,8 \\
\hline 3 & Almaty (Région) & 30049 & 102023 & 49749 & 48,8 & 52274 & 51,2 \\
\hline 4 & Atyrau & 3656 & 14998 & 7250 & 48,3 & 7748 & 51,7 \\
\hline 5 & $\begin{array}{l}\text { Kazakhstan } \\
\text { oriental }\end{array}$ & 8945 & 35069 & 15925 & 45,4 & 19144 & 54,6 \\
\hline 6 & Jambyl & 11123 & 63983 & 31351 & 49,0 & 32632 & 51,0 \\
\hline 7 & $\begin{array}{l}\text { Kazakhstan } \\
\text { occidental }\end{array}$ & 3172 & 14234 & 6902 & 48,5 & 7332 & 51,5 \\
\hline 8 & Karaganda & 12998 & 46273 & 22311 & 48,2 & 23962 & 51,8 \\
\hline 9 & Kostanai & 5891 & 24573 & 11795 & 48,0 & 12778 & 52,0 \\
\hline 10 & Kyzylorda & 3542 & 17314 & 7943 & 45,9 & 9371 & 54,1 \\
\hline 11 & Mangystau & 24460 & 85464 & 34727 & 40,6 & 50737 & 59,4 \\
\hline 12 & Pavlodar & 6175 & 28072 & 13064 & 46,5 & 15008 & 53,5 \\
\hline 13 & $\begin{array}{l}\text { Kazakhstan } \\
\text { septentrional }\end{array}$ & 6439 & 33951 & 14752 & 43,5 & 19199 & 56,5 \\
\hline 14 & $\begin{array}{l}\text { Kazakhstan } \\
\text { méridional }\end{array}$ & 45827 & 155963 & 73438 & 47,1 & 82525 & 52,9 \\
\hline 15 & Ville d'Almaty & 1778 & 6471 & 3162 & 48,9 & 3309 & 51,1 \\
\hline 16 & Ville d'Astana & 1199 & 5661 & 2632 & 46,5 & 3029 & 53,5 \\
\hline & \multicolumn{1}{|c|}{ Total } & 179524 & 706041 & 328259 & 46,5 & 377782 & 53,5 \\
\hline
\end{tabular}

Source : Agence des Statistiques de la République du Kazakhstan, Données : 1991 - 1er octobre 2008 ; Kazak Eli (2009), $24^{\text {th }}$ Nauryz, 5-6.

En outre, des oralman reviennent par leurs propres moyens au Kazakhstan, et ce sont eux qui rencontrent les plus grandes difficultés pour s'installer dans le pays. Mais, 
qu'ils entrent dans le cadre des quotas d'immigration ou qu'ils reviennent spontanément, l'État garantit à tous des conditions avantageuses de réinstallation : aides dans la recherche d'un emploi et d'un logement, attributions de pensions et d'allocations conformes à la législation sociale, bourses pour des études supérieures, offres de terrains constructibles ou agricoles. Les services de l'État organisent aussi des séminaires de formation sur les aspects juridiques et culturels.

\section{CONCLUSION}

La politique officielle de la république kazakhe vis-à-vis de la diaspora ou du rapatriement des oralman a certes évolué, mais elle demeure inchangée dans ses objectifs principaux. Elle œuvre pour l'amélioration des conditions d'accueil et de vie des oralman et celle des Kazakhs vivant au-delà de ses frontières. Certes des lacunes subsistent dans cette prise en charge par les autorités locales notamment, mais cette politique va en sens contraire de ceux qui ont tendance à critiquer les priorités de la politique de l'État kazakh concernant la diaspora, l'irredenta et les rapatriés.

\section{Références bibliographiques}

ABADAN-UNAT Nermin (1985) Identity Crisis of Turkish Migrants. First and Second Generation, in İlhan Başgöz and Norman Furniss Dir., Turkish Workers in Europe. An Interdisciplinary Study, Indiana University Turkish Studies, 5, Bloomington Indiana, pp. 3-22.

Agence des Statistiques de la République du Kazakhstan (2005), Rapport démographique annuel du Kazakhstan, 2004. Rapports annuels, Almaty, Agence des Statistiques de la République du Kazakhstan, 448 p. (en russe).

Agence des Statistiques de la République du Kazakhstan (2007) Rapport statistique du Kazakhstan. 2007, Astana, Agence des Statistiques de la République du Kazakhstan, 494 p. (en russe).

ASHKENASI A. (1990) Nationalism and National Identity, B.; Das arab. Buch, Ethnizitat und Ges, Occasional papers, 23, $250 \mathrm{p}$.

Avrupa Kazak Türkleri Bülteni (1994), Munich, 2.

CASTLES S. and KOSACK G. (1973) Immigrant Workers and Class Structure in Western Europe, London, $268 \mathrm{p}$.

COBBAN Alfred (1969) The Nation State and National Self Determination, New York, 189 p.

Diasporas in International Politics (1986) London, Groom-Helm Publishers, 189 p.

DIENER Alexander (2009) One Home or Two? The Nationalization and Transnationalization of Mongolia's Kazakhs, Washington DC, Woodrow Wilson Center Press, Stanford, Stanford University Press, $405 \mathrm{p}$.

ДӘУЛЕТОВ Т. (2002) Дүниежүзі қ̧азақтары қ̧ауымдастывыңын шежіресі, Алматы, ДҚҚ, 1046.

ДӘУЛЕТОВ Т. (2005) Дүниежүзі қ̧азақ̆тары қ̧ауымдастывыңын шежіресі (29/07/2002 29/09/2005). II kiman, Алматы, ДҚҚ, 115 б.

ДӘУЛЕТОВ Т. (2003) Дүниежүзі қ̧азақтарының II құрылтайы, Алматы, 77 б.

ENGELBREKTSSON U.-B. (1978) The Force of Tradition. Turkish Migrants at Home and Abroad, Göteborg, 309 p.

ESMAN Milton J. (1990) Ethnic Pluralism and International Relations, Canadian Review of Studies in Nationalism, XVII (1-2), pp. 83-88. 
IA Novosti-Kazakhstan (2010), 18 January.

ҚАЗАҚ ЕЛІ (1996) Мамырдың 13-і, с. 2.

ҚАЗАҚ ЕЛІ (1997) Мамырдың 30-ы, с. 5.

KAZAKH ELI (2009) $24{ }^{\text {th }}$ Nauryz, 5-6.

ҚОБЛАНДИН Қ.И., МЕНДІҚҰЛОВА Г.М. (2009) Өзбекстандагы қ̧азақтардың тарихы және бүгінгі дамуы, Алматы, ДҚҚ, 294 б.

КСЁНДЗЫК Н.Н. (1991) Туреикая трудовая иммиграџия в странах Западной Европь (70-80 г2.), Киев, Наукова думка, 108 с.

Law of the Republic of Kazakhstan about Migration (1998) Zakon Respubliki Kazakhstan ot 13 Dekabrya 1997 goda № 204-I. O Migratsii naseleniya, Almaty, 121 p.

Loi de la République du Kazakhstan sur les migrations, du 13 décembre 1997, n²04-I. De la migration de la population (1998) Almaty, $121 \mathrm{p}$ (en russe).

MENDIKULOVA Gulnara (2009) Contemporary Migration Trends between Kazakhstan and China, Proceeding of International Conference "Central Asia-China: Conditions and Perspectives of Cooperation”, 4-5 th June 2008, Almaty, KISI, pp. 158-170.

МЕНДИКУЛОВА Г.М. (2006) Казахская диаспора: история и современность, Алматы, Реиз, $343 \mathrm{c}$.

MENDIKULOVA Gulnara (2000) Repatriation into Kazakhstan: History and Current Situation. International Conference on Central Asia, Toronto, 6, pp. 77-81.

MENDIKULOVA Gulnara (2005) Repatriation of the Kazakhs from Turkey into Sovereign Kazakhstan, Interrelations of Turkey and Central Asia in the Context of Enlarging Europe, Almaty, pp. 257-266 (in Russian).

MENDIKULOVA Gulnara (2004) Some Problems of Adaptation of the Kazakh Repatriates (Oralmans) in Contemporary Kazakhstan, Otan tarikhy, 2, pp. 3-35.

МЕНДИКУЛОВА Г.М. (2000а) Актуальные проблемы диаспорологии в Казахстане, Отан тарихы, 3-4, с. 37-42.

МЕНДИКУЛОВА Г.М. (1997) Исторические судьбы казахской диаспоры. Происхождение и развитие, Алматы, Гылым, 262 с.

МЕНДИКУЛОВА Г.М. (1998) Казахская диаспора и Республика Казахстан: проблемы и перспективы, Казахстан-Спектр, 1, с. 73-77.

МЕНДИКУЛОВА Г.М. (1995) Казахская ирредента в России (история и современность), Евразийское сообщество, 8, с. 70-80.

МЕНДИКУЛОВА Г.М. (1996) Международный симпозиум “Казахская диаспора: проблемы и перспективы”, Вестник Министерства науки-Академии наук Республики Казахстан, 2, c. 75-78.

SHEFFER Gabriel (Ed.) (1986) Modern Diasporas in International Politics, London \& Sydney, Palgrave-Macmillan, $349 \mathrm{p}$.

TAPIA Stéphane de, AKGÖNÜL Samim (Dir.) (2007) Kazakhs, Kalmouks et Tibétains en France, Paris, L'Harmattan (Compétences interculturelles), 273 p.

ФРОЛКИН Н.М. (1975) Трудовая иммиграция во Франции в новейшее время, Киев, Наукова думка, 285 с. 


\title{
Revue européenne des migrations internationales
}

vol. $26-\mathrm{n}^{\circ} 3$ (2010)

Migrations en Asie centrale et au Caucase

Gaëlle Lacaze

\section{Le choix du mouvement : stratégie(s) communautaire(s) des Kazakhs- Mongols}

\author{
Avertissement \\ Le contenu de ce site relève de la législation française sur la propriété intellectuelle et est la propriété exclusive de \\ l'éditeur. \\ Les œuvres figurant sur ce site peuvent être consultées et reproduites sur un support papier ou numérique sous \\ réserve qu'elles soient strictement réservées à un usage soit personnel, soit scientifique ou pédagogique excluant \\ toute exploitation commerciale. La reproduction devra obligatoirement mentionner l'éditeur, le nom de la revue, \\ l'auteur et la référence du document. \\ Toute autre reproduction est interdite sauf accord préalable de l'éditeur, en dehors des cas prévus par la législation \\ en vigueur en France.
}

\section{revues.org}

Revues.org est un portail de revues en sciences humaines et sociales développé par le Cléo, Centre pour l'édition électronique ouverte (CNRS, EHESS, UP, UAPV).

\section{Référence électronique}

Gaëlle Lacaze, « Le choix du mouvement : stratégie(s) communautaire(s) des Kazakhs-Mongols », Revue européenne des migrations internationales [En ligne], vol. 26 - n³ | 2010, mis en ligne le 01 décembre 2013, consulté le 03 janvier 2014. URL : http://remi.revues.org/5233 ; DOI : 10.4000/remi.5233

Éditeur : Université de Poitiers

http://remi.revues.org

http://www.revues.org

Document accessible en ligne sur : http://remi.revues.org/5233

Ce document est le fac-similé de l'édition papier.

(c) Université de Poitiers 


\section{Note de recherche}

\section{Le choix du mouvement : stratégie(s) communautaire(s) des Kazakhs-Mongols}

\section{Gaëlle LACAZE ${ }^{1}$}

T'Asie Intérieure offre un lieu de rencontre de différents peuples turco-

Lmongols. Plusieurs groupes ont ainsi construit des identités d'une grande complexité. Les Kazakhs de Mongolie constituent un exemple parmi d'autres de ces processus. Leur situation de population acculturée est atypique. Les Kazakhs forment un groupe « minoritaire » en Mongolie. Depuis 1991, ils sont invités à quitter la Mongolie pour revenir au Kazakhstan, leur « pays ancestral ». Ils y deviennent des Oralmandar (kz. : sing. Oralman, pl. Oralmandar), des « Rapatriés ». Compte tenu de la complexité des identités qu'ils développent, aujourd'hui, nous devons adopter des conventions terminologiques. Je distingue, dans cet article, les « Kazakhs de Mongolie » de ceux qui ont migré au Kazakhstan après 1990, les « Oralman mongols » et j'utilise l'expression « KazakhsMongols » pour dénommer sans les différencier les membres de ces deux communautés.

Cet article se fonde, entre autres, sur la récente monographie de Diener, One homeland or Two? The Nationalization et Transnationalization of Mongolia's Kazakhs (2009), qui étudie les situations contemporaines vécues par les Kazakhs-Mongols. L'intérêt de cette monographie réside avant tout dans l'originalité de sa démarche, aucun ouvrage n'ayant aujourd'hui été consacré aux Kazakhs-Mongols. En outre, cet ouvrage vient combler la rareté des informations concernant cette population autant dans les sources sur la Mongolie que dans celles sur le Kazakhstan ou dans les rapports des organismes internationaux.

Cet article se base également sur les matériaux que j'ai rassemblés lors de différentes enquêtes menées en Mongolie et au Kazakhstan. Parmi les quelque 465000 Oralman que compterait le Kazakhstan en 2005 (UNDP 2006 : 13), les Oralman mongols

1 Maître de conférences, Université de Strasbourg (détachée d'enseignement à l'Université de Picardie - Jules Verne); gaelle.lacaze@misha.fr 
sont les « laissés pour compte » des différentes études ${ }^{2}$. Lors de l'enquête de terrain que j'ai effectuée en 2002 au Kazakhstan, j'ai rencontré plusieurs représentants d'ONG kazakhes actifs auprès des Oralman, notamment des membres de l'association ASAR créée par des Kazakhs de Mongolie. Les chiffres qu'ils m'ont alors donnés incluent les Oralman venus en dehors du système de quotas. J'ai également récolté différents documents élaborés par les sections locales de l'OIM et du BIT et par divers organismes des Nations Unies.

Les destinées des Kazakhs-Mongols, qu'ils soient Kazakhs de Mongolie ou Oralman mongols, sont intrinsèquement liées aujourd'hui. Cette relation apparait d'emblée dans l'estimation de leur nombre total et dans celui respectif à chaque communauté. Nous commencerons donc par éclaircir plusieurs questions concernant le dénombrement des Kazakhs-Mongols avant et après leurs migrations au Kazakhstan. Nous examinerons ensuite les groupes constitués, d'une part, par les Kazakhs de Mongolie et, d'autre part, par les « Oralman mongols » du Kazakhstan.

L'analyse des identités et des stratégies développées dans ces deux contextes illustre un choix implicite des Kazakhs-Mongols : parcourir les routes transnationales, se mouvoir le long de réseaux « ethniques » de socialisation et d'échange. Les "Oralman mongols » développent aujourd'hui des stratégies qui impliquent des mouvements à travers les sommets de l'Altaï, de larges réseaux de coopération et une conscience communautaire fondée sur une identité kazakhe complexe, politique, territoriale et ethnique. Je finirai par une brève note de recherche, présentée sous la forme d'un épilogue, concernant les migrations actuelles des Mongols de Mongolie.

\section{UNE ESTIMATION DIFFICILE}

En 2005, un rapport de 1'UNDP, Status of Oralmans in Kazakhstan estimait à 80000 le nombre de Kazakhs en Mongolie - soit moins de $4 \%$ de la population totale du pays - et à 71500 le nombre d'Oralman mongols au Kazakhstan (UNDP, 2006 : $8,13)$. Selon ces chiffres, l'UNDP évaluait donc à 151500 le nombre de KazakhsMongols. Or, selon les statistiques mongoles, en 1989, on comptait 120000 Kazakhs en Mongolie, soit $6,1 \%$ de la population, dont plus de $60 \%$ résidaient « à la campagne » (Population of Mongolia, 1994 : 37-38). C'est également ce chiffre qui est retenu par Diener qui estime, en revanche, qu'au total 67000 Kazakhs de Mongolie auraient migré au Kazakhstan entre 1991 et 2005. Il reconnaît, par ailleurs et en accord avec les chiffres

2 Citons, par exemple, le très documenté rapport de la FIDH Kazakhstan/Kirghizstan : exploitation of migrants workers, protection denied to asylum seekers and refugees (2004). Ce rapport mentionne rarement les Oralman mongols alors qu'ils y constituent quantitativement la seconde population d'Oralman après les Ouzbeks, quant à eux régulièrement cités dans ce rapport. De même, les Oralman mongols sont peu évoqués dans le très documenté article de Mendikulova. Comment expliquer cette absence ? Les Oralman mongols se situent à la marge du processus de migration ethnique des Kazakhs ; ils représentent une catégorie interstitielle d'Oralman, liée au statut de leur pays d'origine. La Mongolie n'a pas fait partie de l'URSS, à la différence des pays de l'actuelle CEI d'où émigre un grand nombre d'Oralman. C'était néanmoins une République socialiste, à la différence des pays de provenance des Oralman non-russophones, la Chine faisant exception. 
de l'UNDP, qu'il demeure environ 80000 Kazakhs en Mongolie en 2005. Ce qui élève alors le nombre actuel de Kazakhs-Mongols à environ 147000 (Diener 2009 : 244). Or, les sources mongoles et internationales s'accordent à dire qu'en 2010, il reste plus de 100000 Kazakhs en Mongolie soit $5 \%$ de la population totale du pays. À partir de ces données et si l'on prend également en compte les « Oralman mongols », selon les chiffres Diener et ceux de l'UNDP, les Kazakhs-Mongols compteraient environ 170000 personnes entre la Mongolie et le Kazakhstan. L'estimation du nombre de Kazakhs-Mongols et, par conséquent, celle de leur répartition entre la Mongolie et le Kazakhstan pose donc différents problèmes.

Fin 1995, lors de sa visite en France, M. Dj. Enkhsaïkhan, secrétaire-général du Conseil de Sécurité nationale (Mongol Ulsijn ündesnij ajulgüj bajdlijn dzövlöl), instance dont le rôle est consultatif et qui réunit le président, le Premier ministre et le président du Parlement, reconnaissait que les Mongols ne disposaient «pas de chiffres sûrs concernant le nombre de Kazakhs de Mongolie installés au Kazakhstan. [...] Une partie d'entre eux revient, et les départs semblent diminuer »(Even, 1996). Combien de Kazakhs ont quitté la Mongolie ? Combien en reste-t-il ?

Les problèmes posés par le décompte des Kazakhs-Mongols ne sont pas irrésolubles. La différence entre le décompte de l'UNDP et les chiffres de Diener peut provenir des difficultés à recenser les populations d'Oralman, mobiles et flexibles. Ainsi, en 2004, l'UNDP (UNDP, 2006 : 10) estime que le nombre d'Oralman dépasse de $86 \%$ les quotas autorisés cette année-là. En outre, nous y reviendrons dans la dernière partie de cet article, il faut tenir compte des stratégies migratoires des Kazakhs-Mongols : les départs au Kazakhstan et les retours en Mongolie, dont l'importance est un fait acquis, ainsi que le développement de stratégies orientées vers la mobilité géographique et la flexibilité statutaire. Ces paramètres rendent effectivement difficile le calcul du nombre de KazakhsMongols, car un tiers d'entre eux participerait à plusieurs décomptes démographiques et relevés statistiques, à la fois, en Mongolie, en Russie, en Chine et au Kazakhstan.

\section{LES KAZAKHS DE MONGOLIE}

Au cours des années $1990^{3}$, l'appel à la migration ethnique du gouvernement kazakh, dans le cadre de la politique de rapatriement des « diasporas » kazakhes en vue de « re-kazakhiser » le Kazakhstan ${ }^{4}$, mise en place par le président kazakh N. Nazarbaev, souleva l'engouement des Kazakhs de Mongolie. Nous reviendrons sur les conséquences de cet appel à la migration ethnique dans la seconde partie de cet article. La ou les communauté(s) formée(s) par les Kazakhs de Mongolie ont alors connu une profonde reconfiguration démographique et sociale, interrogeant les identités qu'ils avaient précédemment construites, et qu'il nous faut maintenant décrire.

3 En 1990, les Kazakhs de Mongolie vivaient essentiellement dans la province occidentale de Bajanölgij et dans la région minière de Nalajh, près d'Ulaanbaatar.

4 En 1989, le Kazakhstan comptait seulement $40 \%$ de Kazakhs et $60 \%$ d'autres nationalités, essentiellement des Russes, mais également des Coréens, des Ouzbeks, des Kirghiz, etc. 
La Mongolie comme le Kazakhstan sont nés durant la période socialiste à travers l'enjeu du contrôle du territoire. Actuellement, ces États nations se caractérisent, entre autres, par l'émergence de courants nationalistes. Ainsi, les Kazakhs de Mongolie forment un groupe minoritaire dans un État-nation largement dominé par les «purs Mongols » (mg. : cever mongol), c'est-à-dire, les Mongols Halh. Ils développent aujourd'hui des processus identitaires inscrits dans ces sentiments nationalistes et se caractérisent en tant que Kazakhs-Mongols.

L'histoire de la « horde méridionale » (kz. : orta žuuz) kazakhe, regroupant entre autres les tribus Kereï et Naïman, majoritaires parmi les Kazakhs de Mongolie, s’imbrique à celle des peuples mongols dont ce sont des «voisins » historiquement proches ${ }^{5}$. La majorité des Kazakhs de Mongolie est issue de migrations « volontaires » effectuées entre 1860 et 1880 en provenance de Chine (Diener, 2009 : 264). En Mongolie, rares sont les Kazakhs directement chassés du Kazakhstan lors des purges de Staline ; peu d'entre eux ont migré pour fuir la collectivisation et les famines du XXe siècle. Le lien des Kazakhs de Mongolie aux régions de l'Altaï et aux populations mongoles qui y résident également se retrouve dans les dynamiques identitaires qu'ils ont développées au cours du XXe siècle et dans celles qu'ils déploient en réponse aux possibilités de rapatriement au Kazakhstan. Diener dans la monographie qu'il consacre aux Kazakhs de Mongolie s'interroge sur la nature de cette identité : est-elle « hybride ${ }^{6} »$ ou « diasporique $^{7} »$ ?

Plusieurs éléments tendent à montrer que l'identité des Kazakhs de Mongolie ne se réduit pas à une forme d'hybridité. En effet, les Kazakhs les plus mongolisés vivent dans les régions du centre du pays ; la plupart travaille dans le secteur minier et appartient aux classes sociales moyennes. Dans les campagnes de la province mongole de Bajan-ölgij, en revanche, les Kazakhs âgés parlent peu le mongol et, parmi les jeunes, beaucoup de ruraux n'en connaissent que des rudiments. Les contacts des Kazakhs avec la culture mongole n'entraînèrent donc pas leur acculturation profonde. Aujourd'hui encore, la situation des femmes kazakhes en Mongolie illustre le rigorisme de la culture kazakhe islamisée par opposition à la culture mongole bouddhisée, les femmes mongoles étant censées avoir des mœurs « libres ». Si l'on peut parler d'hybridation des Kazakhs de Mongolie, elle ne concerne donc que l'élite de cette communauté.

Contrairement à ce qu'avance Diener (2009 : 36-40), les Kazakhs ne semblent pas, non plus, développer une « identité diasporique ${ }^{8} »$. Cette conception identitaire

5 On retrouve ces tribus dans l'Histoire secrète des Mongols (Even, 1994), récit hagiographique de la dynastie gengiskhanide. En outre, ils jouent un rôle dans les conflits impliquant les Ö̈rats, leurs voisins mongols localisés en Djoungarie, dans le Xinjiang (Chine).

6 L'hybridité implique la notion d'une pureté originelle, état qui n'intervient jamais dans le cas des populations. Les géographes emploient cependant cette notion pour insister sur l'acculturation réussie d'un groupe diasporique dans un État d'accueil.

7 Selon Diener, la diaspora se définit par plusieurs éléments, dont le mythe du retour et la conception d'une « terre natale » versus « terre d'accueil » (2009: 9-10).

8 Diener invite, lui-même, à nuancer la notion de « diaspora » concernant les Kazakhs de Mongolie car ceux-ci se sont (re)territorialisés en Mongolie. Or, généralement, les groupes diasporiques apparaissent davantage comme « déterritorialisés » et « déplacés » (ibid. : 38). 
implique un processus de (dé)territorialisation. Ce processus n'est pas clair dans le cas de la communauté des Kazakhs de Mongolie. Il implique une redéfinition de la notion de « terre natale » (ibid. : 34). En effet, bien qu'arrivés à la suite de mouvements migratoires dans la province la plus occidentale de Mongolie, les Kazakhs de la " horde méridionale » sont historiquement localisés dans cette région montagneuse et semi-aride caractérisée par la présence de l'Altaï'. Ils pratiquent traditionnellement un élevage extensif, comparable à celui des Mongols de la région ${ }^{10}$. Leurs territoires de nomadisation les ont conduits à séjourner ponctuellement dans la partie orientale de l'Altaï, ne serait-ce que pour échapper aux $z u d^{11}$. La province de Bajan-ölgij ne se trouve pas considérée comme une terre d'accueil différente de la « terre natale » de référence des Kazakhs de Mongolie. Elle se situe, au contraire, dans son prolongement dans le contexte d'une notion de « territoire de nomadisation $»$.

En outre, les Kazakhs de Mongolie n'ont développé un « mythe du retour» au Kazakhstan qu'à la fin des années 1980, quand les mouvements nationalistes émergents ont rendu leur présence en Mongolie difficile et leur installation au Kazakhstan envisageable. Ils n'ont donc que récemment agrémenté leur(s) définition(s) identitaire(s) d'une dimension « diasporique ». Les Kazakhs ne sont pas les seuls « étrangers » en Mongolie où beaucoup de communautés cumulent une identité culturelle de " petit peuple ${ }^{12}$ » et une citoyenneté mongole. La construction de la RPM entraîna la hiérarchisation des peuples mongols, certains se trouvant relégués au rang de «minorité » (mg. : jastan) dans la « nation » (mg. : ündesten) mongole dominée par les Halh ${ }^{13}$. Ainsi, les Mongols Darhad jouissent-ils du même statut que les Kazakhs de Mongolie : ils sont une jastan dans la nation mongole. Pour les Darhad, en revanche, les références nationales et citoyennes relèvent du même registre culturel, la mongolité. Ce n'est pas le cas pour les Kazakhs qui gravitent entre deux cultures proches. Ces derniers n'appartiennent donc pas à la catégorie des « métis » (mg. : erlijz) qui désignent majoritairement les peuples mongols non Halh ou non-gengiskhanides (Bulac, 1998). Plus que d'autres jastan de Mongolie, les Kazakhs

9 Les Kazakhs de Mongolie sont majoritairement arrivés dans la province de Bajan-ölgij depuis la province du Xinjiang (Chine). Les groupes kazakhs qui y demeurent aujourd'hui se situent dans le nord de la province, essentiellement sur les sommets septentrionaux de l'Altaï.

10 Les troupeaux sont dominés par le cheval et comportent de nombreux chameaux. Les parcours de nomadisation alternaient, traditionnellement, entre les hauts pâturages d'hiver et les pâturages estivaux des vallées.

11 Les zud sont des intempéries caractérisées par leurs conséquences sur les troupeaux. Les zud «blancs » ou « noirs », avec trop ou trop peu de précipitation, que ce soient des zud d'été ou d'hiver, entraînent d'importantes pertes de bétail. Ce terme est commun aux langues mongoles et kazakhes.

12 En ex-URSS, un grand nombre de «petits peuples » (rs. : malenkie narody) connaît cette dualité identitaire entre citoyenneté et nationalité.

13 Ce processus a été analysé par Bulac (1998). Son analyse met en évidence, parfois de manière peu objective, le processus nationaliste de construction de la mongolité au cours des XIXe et XXe siècles. 
ont donc développé une sous-culture ${ }^{14}$ qui ne mélange pas les deux cultures de référence, mais préserve les éléments identitaires structurels de la culture nationale dans la culture dominante du pays d'accueil.

La sous-culture des Kazakhs de Mongolie ne correspond pas à une identité transnationale, mais bien comme Diener l'avance en conclusion de son ouvrage (2009: 336), à une série d'identités complexes alliant des appartenances ethniques et territoriales malléables. Les reformulations des identités des Kazakhs-Mongols après la chute des économies socialistes et, surtout, comme nous allons maintenant l'examiner, lors des mouvements migratoires au Kazakhstan, témoignent de leur appartenance à un groupe caractérisé par l'attachement à une culture kazakhe et à un territoire mongol. En Mongolie, cette sous-culture est par ailleurs bien identifiée par les Mongols.

\section{LES ORALMAN MONGOLS}

Entre 1990 et 2000, les Oralman mongols constituaient le groupe de « rapatriés » le plus important au Kazakhstan, environ $35 \%$ des quotas officiels (Diener, 2009 : 234). Ils sont depuis largement dépassés par les Kazakhs d'Ouzbékistan, qui représentaient $72 \%$ de l'ensemble des Oralman en 2006 (UNDP, 2006 : 13). La situation des Oralman mongols est, à plus d'un titre, intéressante. Par exemple, le cloisonnement linguistique des Oralman mongols est différent de celui des autres Oralman non-russophones : Chinois, Afghans, Iraniens, Pakistanais ou Turcs. Les Oralman mongols parlent mieux le kazakh que les Kazakhs kazakhstanais, à l'instar des Oralman chinois ; mais, à la différence de ces derniers, ils connaissent et utilisent le cyrillique, adapté aux langues mongole et kazakhe dans les années 1930. Or, les Oralman non-russophones connaissent les plus grandes difficultés à s'insérer au Kazakhstan ${ }^{15}$; la situation atypique de ceux de Mongolie favorise leur marginalité.

L'immigration des Kazakhs de Mongolie au Kazakhstan après 1990 distingue plusieurs « courants » : la migration de cadres et OQ du secteur minier entre 1991 et 1994 ; le regroupement familial entre 1995 et 1999 ; des migrations internes et le développement d'une communauté transnationale entre 2000 et 2005. Depuis, une nouvelle forme de migration semble apparaître grâce au développement des échanges transfrontaliers, nous $\mathrm{y}$ reviendrons dans la troisième partie de cet article. Invités à se rapatrier au Kazakhstan dans le cadre de contrats quinquennaux dans le secteur agricole, les cadres et OQ kazakhs ont connu une véritable dépréciation statutaire. La plupart d'entre eux connaissait peu l'élevage.

14 Selon Becker dont l'ouvrage Outsiders (1968) est précurseur des études en sociologie de la déviance, une « sous culture » (subculture en anglais) caractérise les communautés rassemblées autour de pratiques « déviantes » et les groupes marginalisés. En Mongolie, l'Islam peut conduire à des pratiques considérées comme déviantes et l'appartenance à la nationalité kazakhe entraîne une stigmatisation importante dans certaines activités politiques et économiques.

15 Tous les rapports concernant les Oralman soulignent l'augmentation des difficultés pour ceux d'entre eux qui ne connaissent pas la langue russe et viennent d'un état hors de l'ex-URSS, car le russe est aujourd'hui la seconde langue vernaculaire du Kazakhstan, parfois la première dans le nord du pays. 
Dès qu'ils l'ont pu, parfois avant la fin de leur contrat, les premiers Oralman mongols ont quitté le secteur primaire. En l'absence de connaissance du russe, ils ont connu un fort taux de chômage. Leur insertion a souvent été problématique dans un pays en crise. Entre $35 \%$ et $70 \%$ des Oralman en âge de travailler seraient au chômage en 2005 (Diener, 2009 : 267), tandis que le nombre national de chômeurs n'excèderait pas $10 \%$ de la population du Kazakhstan, 8,4 \% en 2004 selon l'UNDP (2006 : 15). La deuxième vague de migrants profita du regroupement familial. Elle vient trouver un confort inexistant et des infrastructures publiques déliquescentes en Mongolie. Ainsi sont arrivés des anciens qui souhaitaient mourir sur leur « terre ancestrale » et des enfants rapatriés pour étudier (Diener, 2009 : 266).

Généralement, les Oralman ont été installés dans des régions désertées par d'anciennes « victimes diasporiques » (victim diasporas) de l'ex-URSS (Diener, 2009 : 218). À la suite d'un regroupement familial, la plupart des premiers Oralman a quitté la région initiale de son rapatriement au Kazakhstan pour des raisons de correspondance avec son environnement d'origine et afin de lutter contre l'éparpillement démographique (Ibid : 232-235). Les Oralman mongols se sont regroupés dans l'Est du pays et la région d'Almaty, dans les régions montagneuses de l'Altaï. Se rapprochant des parents restés en Mongolie, ils reconstruisent une sous-culture kazakhe mongole dans un environnement comparable à l'écosystème de Mongolie occidentale. Si un petit nombre d'Oralman mongols s'est effectivement reconverti dans l'industrie minière, la plupart a développé des activités de vente de produits « ethniques » (broderies) ou régionaux (viande ${ }^{16}$ ). Certains servent également d'intermédiaires dans l'approvisionnement du négoce transfrontalier des Kazakhs de Mongolie.

Depuis les années 1990, le rapatriement des « diasporas kazakhes » au Kazakhstan vise l'augmentation du nombre de Kazakhs parmi les citoyens kazakhstanais. Beaucoup de ces rapatriés, surtout parmi ceux issus des pays extérieurs à l'ex-URSS, parlaient encore le kazakh et avaient conservé des savoirs et savoir-faire traditionnels liés au nomadisme pastoral (cuisine, broderie, organisation sociale, etc.) souvent oubliés au Kazakhstan. L'arrivée massive d'Oralman au Kazakhstan entraîna donc un processus de redéfinition de l'« identité kazakhe ». Dans le contexte d'une crise économique profonde et de la restructuration post-socialiste, ce processus de (re)définition identitaire s'est rapidement transformé en sentiment nationaliste et xénophobe.

\section{DES COMMUNAUTÉS EN MOUVEMENT}

Le contexte politique, social et économique n'a pas favorisé l'intégration des Oralman au Kazakhstan. Beaucoup d'Oralman mongols se sont sentis isolés et marginalisés. Ils ont souffert d'une image péjorative attribuée aux " Mongols » par les Kazakhstanais qui les jugent « feignants », " pingres » et « rustres ». Ceux qui sont finalement retournés en Mongolie évoquent leurs difficultés à vivre au Kazakhstan, leur assi-

16 L'élevage extensif reste pratiqué en Mongolie. Ce pays produit et exporte de grandes quantités de viande. En 2006, Bajan-ölgij possédait un abattoir dont les pratiques se conformaient aux règles de l'Islam. La viande débitée était majoritairement exportée vers la Russie et le Kazakhstan. 
milation aux Mongols sur leur «terre ancestrale », les discriminations dont ils ont fait l'objet face à l'émergence du nationalisme kazakhstanais. Ceux qui ont fait le choix de rester n'ont guère demandé la nationalité kazakhe, car ils préfèrent continuer à bénéficier des aides et des allocations attribuées aux « rapatriés » par le gouvernement kazakh ou de celles octroyées à ses ressortissants par le gouvernement mongol.

Les premiers Oralman mongols ont connu de nombreuses difficultés socioéconomiques. La venue des membres de leur famille lors de la seconde vague de migration a favorisé leur reconversion dans les secteurs du commerce transfrontalier et du petit négoce. En effet, en sortant de leur solitude et de leur isolement, les premiers Oralman mongols ont pu ouvrir des comptoirs de vente en Mongolie, à Bajan-ölgij, avec des produits importés du Kazakhstan ou au Kazakhstan avec des produits de Mongolie ou de Chine. Pour ce faire, la citoyenneté mongole est indispensable car les Mongols n'ont pas besoin de visa pour voyager dans les régions frontalières de la Russie et de la Chine.

Depuis les années 2000, les Oralman mongols se spécialisent dans des activités transnationales (Diener, 2009 : 335). Nombre de mes informateurs kazakhs de BajanÖlgij ont développé des stratégies commerciales transnationales entre la Mongolie, la Russie, le Kazakhstan et la Chine. Lors de l'enquête de terrain que j'ai conduite dans cette province de Mongolie en 2002, j'ai rencontré beaucoup de négociants transfrontaliers qui tenaient, en famille, des étals sur les marchés. Durant l'hiver, l'époux parcourait les routes de Russie et de Chine pour approvisionner les étals tenus par les femmes sur les marchés mongols ou russes. Grâce à la collaboration des membres de leur famille, certains avaient ouvert deux étals, l'un en Mongolie et l'autre dans la province russe de Gorno-Altaj.

En 2006, les plus riches de ces négociants avaient élargi leur réseau d'activités grâce à des membres de leur famille « rapatriés » au Kazakhstan. Certains déployaient des « stratégies de migration temporaire ». Au sein d'un même lignage, des parents migrent au Kazakhstan et s'enregistrent comme Oralman. Une fois épuisé le système d'aides destinées aux Oralman, ils rentrent en Mongolie et un autre membre du lignage prend leur place. Cette stratégie permet de profiter du système d'aide au rapatriement sans perdre le bénéfice des investissements effectués localement en Mongolie et au Kazakhstan. Ces «migrations alternées » sont planifiées entre les membres d'un même lignage qui développe ainsi une stratégie collective pour déployer leurs activités commerciales transfrontalières tout en bénéficiant des aides qui leur sont attribuées. Ces stratégies pourraient expliquer l'excès de 30000 personnes dans le décompte des Kazakhs-Mongols de Mongolie et du Kazakhstan.

Depuis 2000, une tendance nouvelle se repère dans la pérennisation des stratégies transnationales des Oralman mongols. En Mongolie et au Kazakhstan, les KazakhsMongols entretiennent aujourd'hui des liens « ethnico-territoriaux » en utilisant - voire en les (re)construisant - leurs réseaux de parenté présents en Chine et en Russie. Ils se distinguent par une « stratégie de mouvements », privilégiant les relations transnationales, le commerce transfrontalier et la mobilité entre différents lieux au sein d'un réseau de parenté. Ils confirment, par conséquent, l'existence d'une sous-culture spécifique à leur communauté. 


\section{ÉPILOGUE}

On ne peut conclure, même temporairement, concernant les recompositions identitaires des Kazakhs-Mongols, car ils construisent et reconstruisent en permanence de nouvelles stratégies communautaires. Nous proposons un épilogue à cette analyse dans la comparaison avec certaines des stratégies migratoires des Mongols de Mongolie. À l'instar du Rapport sur le développement humain en Mongolie (UNDP, 2003 : 43), il faut déplorer l'absence de travaux publiés sur les migrations actuelles des Mongols. La Corée du Sud, le Kazakhstan et la République tchèque constituent leurs destinations de prédilection. On peut interroger cette absence. Provient-elle du faible nombre de personnes concernées par ces migrations ? De la difficulté de leur investigation, les locuteurs de mongol s'intéressant à des problématiques plus locales que mondialisées ?

Si la presse mongole évoque souvent la question des migrations, c'est pour mettre en évidence les difficultés et les situations problématiques vécues par certains migrants mongols. Le regard de la presse locale est biaisé par un sentiment nationaliste qui déprécie les migrants, considérés comme ayant « fui » les difficultés du pays. La question des migrations contemporaines des Mongols préoccupe les spécialistes occidentaux de la Mongolie, car elle se trouve au cœur de l'actualité mongole ; mais personne n'a encore tenté d'en faire une analyse exhaustive. En revanche, les scientifiques asiatiques, en particulier les Coréens et les Japonais, sont à l'œuvre et tout laisse à croire que différents travaux sont en cours de publication.

Depuis la restructuration post-socialiste des années 1990, les Mongols ont utilisé leur situation « $\operatorname{tampon}^{17}$ », et les privilèges qui y sont liés, en particulier, la relative simplicité des formalités administratives nécessaires pour séjourner en République Fédérale de Russie, dans quelques pays de la CEI ou en RPC. Par exemple, entre 1991 et 2001, ce sont en majorité des Mongols qui ont approvisionné la Russie en produits manufacturés chinois car ils n'avaient pas besoin de visa pour aller en Chine et en Russie ${ }^{18}$. Les « années d'or » $\mathrm{du}$ « négoce à la valise » $\left(\right.$ najma $\left.^{19}\right)$ ont ouvert des voies commerciales jusqu'en Europe de l'Est. Les Mongols se sont, en effet, brièvement spécialisés dans le convoi de voitures « de seconde main », achetées sur le marché allemand et revendues en Mongolie. Les « routes transnationales » de la Mongolie vers certains de ses voisins voient toujours défiler un nombre important de négociants mongols. Leurs trajets s'arrêtent aujourd'hui aux régions frontalières, car les formalités de séjour libre ont été limitées par des accords bilatéraux conclus au début des années 2000 entre la Mongolie et ses deux voisins, la Chine et la Russie (Lacaze, 2010).

À la différence des Kazakhs, les Mongols n'ont pas l'opportunité de migrer de manière pérenne dans un autre État voisin. Ni la Mongolie, ni la Chine, pas plus que la

17 La Mongolie occupe la place d'État tampon entre la Russie et la Chine.

18 Soulignons l'assimilation des négociants mongols à des Chinois dans l'ouvrage de Tinguy (2004: 409-445).

19 Le terme mongol najmaa dérive du terme chinois maimai qui signifie : le négoce, le commerce. En mongol, le najmaa désigne en particulier le «négoce à la valise » caractéristique de la période de restructuration post-socialiste. 
Russie n'entretiennent les aspirations pan-mongolistes émergentes depuis 1990. Aucun de ces États n'a appelé les Mongols au rapatriement. Les Mongols, néanmoins, migrent de manière temporaire dans les régions frontalières de la Chine ou de la Russie où ils y jouissent d'un droit de séjour limité dans le temps. Ainsi, on compte plusieurs milliers de migrants «temporaires permanents » à Erlian-Ereen, ville située sur la ligne ferroviaire du trans-mongol, à la frontière chinoise. Ces Mongols sont des migrants temporaires, car ils n'ont qu'un droit de séjour de trente jours à la frontière sino-mongole. Ils résident néanmoins à Ereen de manière permanente en sortant de Chine une fois par mois. Ils font mensuellement un aller-retour au poste frontière de Zamyn-üüd afin de renouveler leur droit de séjour à Ereen, en Chine (Lacaze, 2010).

Il faut, pour finir, mentionner les migrations mongoles dans les pays « riches ». Les destinations de prédilection des Mongols sont la Corée du Sud, l'Europe, le Japon et les États-Unis. N'ayant pas d'informations précises sur ces deux derniers pays, nous n'évoquerons que la Corée du Sud et l'Europe. Ces deux exemples illustreront la diversité des trajectoires migratoires actuelles des Mongols. À la fin des années 1990, la Corée du Sud offrait une destination migratoire appréciée des Mongols. En 2002, près de 20000 ressortissants travaillaient illégalement dans les secteurs de la construction et des services. Depuis, plusieurs accords bilatéraux visent à régulariser les Mongols de Corée. Ainsi, en 2008, les gouvernements des deux États créent une assurance médicale spéciale pour les immigrés. Le gouvernement coréen a organisé plusieurs campagnes de régularisation et favorisé les contrats de travail destinés aux Mongols. En revanche, depuis 2008 et la crise internationale, il tente de réduire les migrations de travail et freine, en particulier, l'arrivée de nouveaux migrants en provenance de Mongolie. En 2010, la situation des Mongols en Corée du Sud est mieux encadrée qu'auparavant, tandis que le nombre de nouveaux immigrants est régulé.

Comparativement à la Corée, les Mongols sont peu nombreux en Europe. Il y en aurait plusieurs milliers, mais il reste difficile de connaître leur nombre exact, car la majorité y réside illégalement. Les migrations des Mongols vers l'Europe sont essentiellement temporaires et, essentiellement, à des fins de formation ou de coopération. Parmi les résidents permanents, beaucoup de Mongol-es sont marié-es à des ressortissants européens. D'autres ont bénéficié d'accords universitaires et sont demeurés dans leur pays d'accueil une fois finies leurs études. C'est, par exemple, le cas de la majorité des membres des communautés mongoles d'Allemagne et de République tchèque ${ }^{20}$ arrivée durant les années 1990. Ces deux communautés jouent, aujourd'hui, le rôle d'intermédiaire dans les réseaux qui organisent les nouvelles migrations des Mongols sur le territoire de l'Union européenne. Ainsi, la plupart des résidents mongols illégaux sur le territoire de l'UE a emprunté la route qui mène en bus jusqu'à Prague ${ }^{21}$, avant de se rendre quelque temps en Allemagne. De là, les migrants se dirigent vers d'autres pays, principalement, la France,

$20 \mathrm{Au}$ sein de l'UE, la récession a particulièrement touché les populations migrantes en 2008-2009. Plusieurs pays ont proposé des « aides aux retours 》. En République tchèque, près de 5000 personnes en ont bénéficié, principalement des Mongols et des Vietnamiens.

21 En 2008, le trafic de « visa tchèque » faisait encore florès sur le marché noir d'Ulaanbaatar et dans l'organisation d'arnaques liées à l'émigration illégale au départ de la Mongolie. Alors même que la République avait adhéré à l'espace Schengen ! 
la Belgique 22 ou les Pays-Bas, ainsi que la Suède et le Danemark. Ils essaient également, via l'espace Schengen, d'atteindre la Suisse et le Royaume-Uni.

Les citoyens mongols seraient moins d'un millier à vivre en France : une centaine d'étudiants accueillie chaque année, une centaine de Mongol-es marié-es à des ressortissants français et plusieurs centaines de migrants illégaux. Ces derniers sont restés en France après l'expiration de leur visa ; rares sont ceux entrés illégalement sur le territoire. Les quelques expertises, traductions et séances d'interprétariat que j'ai effectuées auprès de migrants mongols résidant de manière illégale en France indiquent qu'une partie d'entre eux vit en volant dans des magasins de luxe. Un réseau « maffieux » s'est structuré autour de cette activité : volés sur le territoire français, les produits de luxe - essentiellement vêtements et parfums - sont revendus sur le marché néerlandais. Pour se prémunir de la répression administrative et policière en France et afin de palier à la crise du logement qui y sévit, les Mongols illégaux résident en Belgique. Le réseau assure quotidiennement le transport des personnes de la Belgique vers la France et le transport des marchandises de la France vers les Pays-Bas. Cette activité fait aujourd'hui florès à tel point qu'en Mongolie, les Mongols de France sont réputés pour être des « voleurs ».

Certains des Mongols qui résident de manière illégale en Europe développent donc un réseau « ethnique » de commerce transnational. Ils assurent l'approvisionnement, le transport et la vente des marchandises, en l'occurrence des produits volés à l'industrie du luxe. À chaque étape du parcours d'acheminement des produits, une communauté des ressortissants mongols sert d'intermédiaire. Cette activité répréhensible s'organise de manière comparable au commerce transfrontalier des Kazakhs-Mongols, quant à lui, plus ou moins légal. Elle renvoie plus largement au commerce transfrontalier développé par les citoyens mongols (Lacaze, 2008). Ainsi, quelques Mongols et Kazakhs de Mongolie se spécialisent dans des activités comparables de commerce itinérant et transfrontalier. Développé et répandu dans les anciens pays socialistes, le " commerce à la valise », ethnique et transfrontalier, devient une véritable « spécialité locale » des citoyens mongols qui bénéficient de droits privilégiés de séjour et d'entrée en Chine et en Russie. Ces derniers utilisent leur réseau élargi de parents, en les reconstruisant ou les réinventant, et profitent ainsi de la dispersion géographique des peuples mongols. Les Kazakhs-Mongols semblent développer les mêmes stratégies.

22 En 2008, 119 citoyens de Mongolie ont bénéficié de l'« aide au retour » accordée par le gouvernement belge, tandis que onze d'entre eux étaient expulsés de force (Réseau Européen des Migrations, 2009 : 18). Plus d'une centaine de ressortissants mongols sont arrêtés en Belgique chaque année depuis 2005. Aucun des ressortissants mongols n'a fait de demande d'asile en Belgique (ibid. : 13-14). Plusieurs d'entre eux, en revanche, sont connus des bureaux français de l'OFPRA. 


\section{Références bibliographiques}

ANN (2008) Mongolie, http://www.tlfq.ulaval.ca/AXL/asie/Mongolie.htm, consulté le 03/11/2010. BECKER Howard S. (1968) Outsiders : études de la sociologie de la déviance, Paris, Métailié, $247 \mathrm{p}$.

BULAC Uradyn (1998) Nationalism and hybridity in Mongolia, Oxford, Clarendon Press, 272 p.

DIENER Alexander C. (2009) One homeland or Two? The Nationalization and Transnationalization of Mongolia's Kazakhs, Chicago, Stanford University Press, 405 p.

EVEN Marie-Dominique et POP Rodica (trad.) (1994) Histoire secrète des Mongols (Mongghol-un ni'uca tobciyan) Chronique mongole du XIIIe siècle, Paris, Gallimard, $349 \mathrm{p}$.

EVEN Marie-Dominique (1996) Un entretien avec Dj. Enkhsaïkhan sur la politique extérieure de la Mongolie, Anda, 20, http://www.anda-mongolie.com/propos/politique/enkhsai20.html, consulté le $03 / 11 / 2010$.

FIDH (2009) Kazakhstan/Kirghizstan: Exploitation of Migrants Workers, Protection Denied to Asylum Seekers and Refugees, Rapport n530a Oct 2009, Paris, 89 p.

LACAZE Gaëlle (2008) Mise en rythme d'une esthétique du pouvoir. Les perceptions esthétiques dans le contexte mongol et sibérien, Actes de la Journée d'étude «Que sont les perceptions esthétiques dans un contexte mongol et sibérien » (20/10/07), Réseau-Asie-CEMS, Paris, http:// www.reseau-asie.com/congrès/\%20Journée\%20d'études/document\%20555.

LACAZE Gaëlle (2010) "Run after time": the roads of suitcase traders, Asian ethnicity, 11 (2), pp. 191-208.

RÉSEAU EUROPÉEN DES MIGRATIONS. Point de contact belge (2009), Programmes et stratégies en Belgique pour le retour volontaire assisté et la réintégration dans un pays tiers, Bruxelles, $37 \mathrm{p}$.

TINGUY Anne (de) (2004) La grande migration : la Russie et les Russes depuis l'ouverture du rideau de fer, Paris, Plon, $662 \mathrm{p}$.

UNDP (2006) Status of Oralmans in Kazakhstan. Overview, Almaty, UNDP, 31 p.

UNDP (2003) Human Development Report. Mongolia, Ulaanbaatar, UNDP, 79 p. 


\title{
Note de recherche
}

\section{The Impact of Labour Migration on Human Capital: The Case of Tajikistan}

\author{
Saodat OLIMOVA ${ }^{1}$
}

\section{INTRODUCTION}

The UN High-level Dialogue on International Migration and Development in September 2006 and the Global Forums on Migration and Development have admitted the connection between migration and development. Effective management of labour migration requires the adjustment of migration for the goals of the overall economic development and re-alignment of migration flows with capital flows, technology transfers and policies for expansion of human capital, encouragement of entrepreneurship, and retention of skilled human resources.

Moreover, as some countries demonstrate the variety of patterns of migration management and distinct economic and socio-cultural factors make the positive impact of labour migration on economic development highly questionable. To understand the connection between labour migration and economic development it is necessary to look at the impact of labour migration on various aspects of economic development in different countries. For this reason the focus of this article is made on the impact of labour migration on human capital in Tajikistan.

Tajikistan is chosen to serve as a case study of the impact of labour migration for several reasons. First of all, being a part of the Soviet Union, Tajikistan has been experiencing structural changes in the process of transition from the Soviet economy. Therefore, the impact of labour migration on economic development is particularly important, since we have an opportunity to witness the interaction of migration and other indicators of economic development in the context of the general process of economic transformation. Secondly, Tajikistan is one the countries which has been experiencing an extremely high level of labour migration over the past decade.

1 Research Center SHARQ (ORIENS), 7 Bofanda Street, Ap. 9, Dushanbe, Tajikistan, p\o 734042, TellFax: +(992-37) 22189 95; sharq@tajik.net, s_olimova@mail.ru 
Tajikistan, along with other Central Asian republics, underwent deep modernization in the Soviet period. One of the outcomes of this modernization was the high level of human capital in Tajikistan. Most of the works on economic development agree on the notion that the high level of human capital (and education) is one of the determinants of economic development. Given this fact, it is interesting to see the connection between the development of human capital in the post-Soviet Tajikistan and labour migration. The main questions to be answered are what are the dynamics of human capital development in the process of economic transformation in Tajikistan and what is the impact of labour migration on the development of human capital.

In this work we define human capital as a set of skills and competences possessed by the labour force and which have a direct positive effect on the level of economic development. In this context we made a particular emphasis on the analysis of formal educational levels, on-the-job training, and other sources of acquisition of professional skills. Other determinants of human capital such as health care, nutritional levels, life expectancy have been omitted from this study, since these determinants of human capital do not have a direct positive effect on economic development.

\section{METHODOLOGY}

The lack of clear understanding of the impact of labour migration on education primarily stems from methodological issues. Most of the empirical quantitative studies of labour migration are based on household surveys. However, this type of data is not a good source of the analysis of dynamics of determinant of human capital and as such cannot explain the evolution of the variables in question over time.

At the same time, the qualitative methods, which are used mostly in anthropological studies, give enough information to analyze a phenomenon in depth, but cannot serve as a ground for analysis of aggregate behavior of labour migrants or migrant households. Therefore, the mixed methodology used in this study is the most relevant because it allows us to employ an interdisciplinary approach to study the problem.

We employ data from a variety of sources, including official statistics, data from the Living Standards Measurement Survey of 2007 (TLSS, 2007), the Labour Force Survey (SCSRT, 2006), data from the European Training Foundation (ETF) Labour Market Analysis in Tajikistan (Quddusov, 2009), UNDP Informal Economy Survey (Olimov, 2007). We also use data collected by the author of this study in the following research projects: Asian Development Bank (ADB) Labour Migration and Poverty Survey (Brown, Olimova and Boboev, 2008), the Human Capital Development Study in Tajikistan conducted by the European Training Institute and SHARQ Research Center in 2009. In addition, we employ results of face-to-face interviews with migrants and employers in November 2008, Tajikistan.

There are a number of studies in different countries, which focus on the impact of migration on the human capital. Most of these studies conclude that migration has a positive impact on the human capital by lowering the extreme poverty levels and expanding the income 
level through remittances from migrants abroad. At the same time, a number of studies show that the positive impact of labour migration on income growth may not have a simultaneous positive effect on the level of human capital (see, for example, De Haan, 2005).

Usually, it is hypothesized that labour migration has a positive impact on human capital, because money remittances from labour migrants are spent on education of their household members, which raises the overall level of education in a country. McKenzie and Rapoport (2006) find that labour migration affects the level of education through three different channels: 1) direct income effect through money remittances, 2) direct substitution effect on demand for child labour (labour migration lowers supply of adult employees and therefore increases demand for child labour), and 3) indirect effect on the expected earnings (if expected wage in destination country exceeds wages in source country, the cost of staying at school for one extra year (in terms of foregone wage) in source country is high, and potential labour migrants have stronger incentives to leave schooling in return for an earlier opportunity to find employment in the destination country). While migration has positive impact on human capital through the first channel, the impact of migration on human capital through the second and third channels is negative. In addition, labour migration may have negative impact on education through higher absenteeism of school children, whose parents are abroad and cannot control school attendance.

Nevertheless, a number of empirical studies show that money remittances have positive impact on education in migrant household as opposed to household without migrants (see Cox Edwards and Ureta, 2003; Hanson and Woodruff, 2003).

In particular, Cox Edwards and Ureta (2003) find that monetary remittances are positively correlated with school attendance and negatively correlated with school dropout rates. Conversely, McKenzie and Rapoport (2006) find that migration is negatively correlated with school attendance rates and overall education level. These authors find that the negative impact of migration on education due to higher demand for child labour overweighs the positive impact of migration due to money remittances and higher income levels. This study along with others show that aside from purely economic factors the impact of migration on education and human capital depend on a variety of social and cultural factors, including household structure (Hanson and Woodruff, 2003; Acosta, 2006). For instance, some studies show children in remittance receiving households have more opportunities to attend private schools and obtain professional education. Additional income from remittances can cover expenses on private education and help keep children from entering labour market early (Hanson and Woodruff, 2003; Thieme, 2009).

Thus, we can summarize the findings of other studies in the following manner. On the one hand, we find that children from remittance receiving household have easier access to education, since remittances can be spent on school supplies, books, and other education-related items. In addition, some migrants use their earnings to finance attendance of their children in universities. On the other hand, due to absence of family members, migration leads to higher school absenteeism, lower academic success, and higher dropout rates of children in migrant households. This leads to the worsening of the quality of acquisition of education and lowers the educational level overall. 
In the context of this summary it is interesting to look at the specifics of educational system of Tajikistan. In the Soviet era Tajikistan enjoyed high levels of equity in access to education and the virtually universal coverage of the education system. In the transition period since 1991, the education attainment levels and literacy levels in Tajikistan remained relatively high, even despite the civil conflict and the general impoverishment of population. Nevertheless, some negative tendencies in the dynamics of human capital development manifested themselves in this period.

Table 1: Main Demographic Indicators in Tajikistan

\begin{tabular}{|l|c|}
\hline Total population (Millions) & $7.28(2008)$ \\
\hline Annual growth rate of population (\%) & $2.0(2006-2008)$ \\
\hline Literacy level among adults (\%) & $99.6(2007)$ \\
\hline Share of population residing in urban areas & $26.4(2007)$ \\
\hline Share of population subsisting on less than USD 1.25 a day (\%) & $21.5(2004)$ \\
\hline
\end{tabular}

Source: State Statistical Committee of Tajikistan, www.stat.tj/english/database.htm

The general trends in the post-Soviet people resulted in the lower level of education of young people in comparison to their parents, significant drop in the quality of education and a higher inequity in access to education for certain social groups.

Table 2: Demographic Indicators by Level of Schooling

\begin{tabular}{|c|c|c|c|c|c|c|}
\hline & 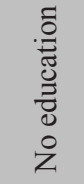 & 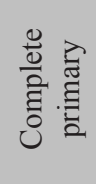 & 造 & 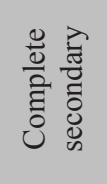 & 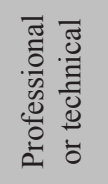 & 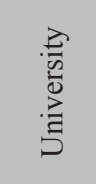 \\
\hline $\begin{array}{l}\text { Share of population aged } \\
\text { above } 15 \text { in } 1989\end{array}$ & $0.0 \%$ & $0.0 \%$ & $25.2 \%$ & $52.1 \%$ & $13.1 \%$ & $10.6 \%$ \\
\hline Men only & $0.0 \%$ & $0.0 \%$ & $20.1 \%$ & $50.9 \%$ & $13.6 \%$ & $10.3 \%$ \\
\hline Women only & $0.0 \%$ & $0.0 \%$ & $22.1 \%$ & $53.6 \%$ & $8.5 \%$ & $6.6 \%$ \\
\hline $\begin{array}{l}\text { Share of population aged } \\
\text { above } 15 \text { in } 2000\end{array}$ & $0.0 \%$ & $5.9 \%$ & $19.1 \%$ & $57.5 \%$ & $6.6 \%$ & $8.9 \%$ \\
\hline Men only & $0.0 \%$ & $5.0 \%$ & $17.0 \%$ & $59.0 \%$ & $8.0 \%$ & $11.0 \%$ \\
\hline Women only & $0.0 \%$ & $7.0 \%$ & $22.0 \%$ & $60.0 \%$ & $6.0 \%$ & $5.0 \%$ \\
\hline $\begin{array}{l}\text { Share of population aged } \\
\text { above } 15 \text { in } 2007\end{array}$ & $0.7 \%$ & $8.7 \%$ & $22.1 \%$ & $48.1 \%$ & $11.0 \%$ & $8.8 \%$ \\
\hline Men only & $0.5 \%$ & $7.1 \%$ & $17.7 \%$ & $45.9 \%$ & $15.7 \%$ & $13.2 \%$ \\
\hline Women only & $0.9 \%$ & $10.1 \%$ & $26.1 \%$ & $51.4 \%$ & $6.7 \%$ & $4.8 \%$ \\
\hline
\end{tabular}

Source: TLSS, 2007 
Table 2 presents the level of education of individual of ages 15 and above according to official data sources (census data of 1989 and 2000 and Tajik Living Standards Survey (TLSS, 2007). Although the share of individuals without education in the census of 1989 and 2000 was 0 percent, it raised to $0.7 \%$ in 2007 according to the TLSS 2007 data.

Despite the small number of people with no education $(0.7 \%)$, there is an increasing trend in the number of those with no education across different age groups. Conversely, the share of people with higher education and professional education fell down in $1989-2007$ by $1.8 \%$ and $2.1 \%$ respectively. The biggest changes are observed in the educational attainment levels of women: the share of women with university and professional education dropped. We can also observe that as opposed to the Soviet period in 2007 the share of women of ages 15 and above with only primary education rose to $10.1 \%$.

When studying the issue of human capital development, it is necessary to address the labour force participation rates. According to the data from the Tajik Living Standards Survey (TLSS, 2007), 43.8\% of the total population was economically active in 2007 , with $58.1 \%$ of men economically active to $31.1 \%$ of women. The analysis of educational attainment shows that $0.35 \%$ of the economically active population have no education at all, while $2.97 \%$ have only primary education. Meanwhile, $15.1 \%$ of the labour force has incomplete secondary education and $49.59 \%$ have complete secondary education, while only $16.44 \%$ have technical or professional education and $14.64 \%$ have higher education. More than $68 \%$ of those with technical or professional and higher education are economically active against $45 \%$ of those with only secondary education. Thus, the acquisition of technical or professional education is associated with an increase in participation by $23 \%{ }^{2}$

These figures demonstrate that while the general quality of the labour force is relatively high (as shown by the small number of people with no education or only basic schooling) only a third all members of the labour force have professional training or higher. Meanwhile, the participation rate for individuals with professional training or higher education is significantly higher than for those with only secondary education.

Moreover, there is a discrepancy between the number of years of schooling and the poor range of skills acquired. Although students attend school for many years, their skills and knowledge fall far below expected levels of the state standard of education.

The falling quality of education is especially noticeable for groups such as rural residents, the poorer sectors and girls. The variable quality of education leads to rising inequity in access to education as poor input at the basic level can bar entry to higher level tuition. Women and rural residents, who find it hard to access schooling and receive low quality education, also have a weaker rate of participation in the labour market.

What are the reasons which led to the deterioration of human capital in Tajikistan? To answer this question we first need to characterize the labour market in Tajikistan in the first decade after 1991.

2 Calculation of author based on TLSS 2007. 
The collapse of the Soviet Union led to the breakup of inter-industry links, the closure of many enterprises and a sharp rise in unemployment. The de-industrialisation process led to a fall of employment in industrial production from $21 \%$ in 1991 to $8 \%$ in 2003 (SCSRT, 2006: 88) and expansion of unemployment. Most of the new unemployed found employment in agriculture, the retail trade, services and household production or stopped looking for job completely.

At the same time, the incomes of population also fell. In 1990 per capita income in Tajikistan was $\$ 1,050$ US. However, in two years it dropped by $50 \%$ reaching $\$ 480$ US per capital in 1992, and in 10 years it dropped even further reaching \$150 US in 2001 (SCSRT, 2006: 116). The unemployment caused by structural changes in the domestic economy in transition period as well as expansion in poverty rates serves as the driving forces behind labour migration to external labour markets. ${ }^{3}$

\section{MIGRATION AND MIGRANTS}

International migration has much stronger impact on the level of human capital in Tajikistan. A large percentage of the labour force in Tajikistan has been employed abroad as temporary labour migrants since the trend began in 1994-1995. A comparative look at international figures demonstrates the scale of Tajik involvement in international labour migration. TLSS 1999 showed $1.5 \%$ of households had migrant workers and the figures grew steadily until 2004, when the increase accelerated, reaching 5\% in TLSS 2007. Data from the Ministry of Labour give the total number of labour migrants as 224.000 in 2003, 254.000 in 2005, 727.000 in 2007 and 805.000 in $2008 .{ }^{4}$

Brown, Olimova and Boboev (2008) find that 37.3\% of households had at least one migrant member. The authors find the number of temporary migrants from Tajikistan at about 700000 people, more than 500000 of whom were working in Russia. In autumn 2008 - spring 2009, the number of labour migrants shrank by $25 \%$ or around 120000 people ${ }^{5}$ as Tajikistan experienced a wave of return migration for the first time. These developments were largely due to the world financial crisis and the sharp decline in economic activity in the Russian Federation.

\section{The Profile of Migrants}

To understand how labour migration affects the level of education and human capital, we first have to look at the profile of migrants and in particular at the socialeconomic characteristics of migrants.

3 Although the unemployment rate has decreased over the past 10 years, it still remains quite high at 13\% (Quddusov, 2009).

4 See Olesya Bobovich, "One million working places and no migration” VD 15.10.2009.

5 Data provided by the Migration Service of the Ministry of the Interior of Tajikistan. 
Table 3: Socio-demographic Characteristics of Migrants (\% of the total number of migrant household members in the category)

\begin{tabular}{|l|c|}
\hline & All migrant household members $(\mathrm{N}=1,658)$ \\
\hline Capital & 5.1 \\
Other urban areas & 18.9 \\
Rural areas & 76.0 \\
\hline Male & 93.2 \\
Female & 6.8 \\
\hline 15 years old or younger & 0.4 \\
$16-24$ & 26.5 \\
$25-34$ & 40.2 \\
$35-44$ & 24.5 \\
$45-54$ & 7.8 \\
$55-64$ & 0.5 \\
65 years or older & 0.1 \\
\hline No formal education & 0.4 \\
Primary & 0.9 \\
Secondary & 76.2 \\
Tertiary & 22.3 \\
Post-graduate & 0.2 \\
\hline Unmarried & 39.5 \\
Married & 60.0 \\
\hline
\end{tabular}

Source: Brown, Olimova and Boboev (2008)

The data from Brown, Olimova and Boboev (2008) presented in table 3 indicates that labour migrants from Tajikistan are mostly male (93\% of all migrants), that young men comprise the bulk of these, (66.7\% of all migrants are between $16-34$ years old) and that $76 \%$ come from rural areas. The level of education of labour migrants is higher than the average in Tajikistan; $22.3 \%$ of migrants have higher education and $76.2 \%$ have secondary education.

Table 4 also suggest that unemployment is the main factors driving people to look for higher-paying jobs abroad. More than $60 \%$ of migrants were unemployed in their home country although only $15.6 \%$ were unemployed and were looking for jobs at home. Meanwhile, $10.8 \%$ of the Tajik population was engaged in unpaid work within their household there were three other equally sized groups of students $(6.0 \%)$, the selfemployed $(7.2 \%)$ and those employed in the private sector $(8.4 \%)$. Civil servants formed the smallest group of the employed at $3.6 \%$. 
Table 4: Occupation of Migrants in Tajikistan and Abroad (in \%)

\begin{tabular}{|l|c|c|}
\hline & $\begin{array}{c}\text { In home country } \\
\text { (Base: migrants who } \\
\text { worked in the home } \\
\text { country in 2006: N=83) }\end{array}$ & $\begin{array}{c}\text { Abroad } \\
\text { (Base: all migrants; } \\
\mathrm{N}=1,698)\end{array}$ \\
\hline Self-employed & 7.2 & 19.8 \\
\hline Entrepreneur with hired worker(s) & 0.0 & 4.2 \\
\hline Employed in public sector & 3.6 & 9.3 \\
\hline Employed in private sector & 8.4 & 59.0 \\
\hline Unpaid family work & 10.8 & 1.5 \\
\hline Unemployed (looking for work) & 15.7 & 1.8 \\
\hline Unemployed (not looking for work) & 45.8 & 1.4 \\
\hline Student & 6.0 & 0.9 \\
\hline Retiree with pension & 0.0 & 0.5 \\
\hline Others & 2.4 & 1.5 \\
\hline
\end{tabular}

Source: Brown, Olimova and Boboev (2008)

The figures reflect the long-term migrant status of much of the population as $45.8 \%$ were unemployed in their home country and not looking for a job, representative of the fact that most seasonal workers will return home during the winter but will not seek work.

According to data from Brown, Olimova and Boboev (2008), migrants abroad are prevalently occupied in construction, amounting to $74.1 \%$. Other employment sectors involve the following percentages of migrants: wholesale and retail trade $10.8 \%$, industry $4.8 \%$, agriculture $5.4 \%$, and other sectors (services, education, etc.) $4.8 \%$. Most migrants are employed on a seasonal basis in Russia returning to Tajikistan in the winter.

\section{Destination Countries}

Although, geographically, migration flows from Tajikistan encompass several Commonwealth of Independent States (CIS) countries, labour migration is entirely focused on the Russian Federation ( $97 \%$ migrants). Small numbers of migrants from Tajikistan are employed in other countries such as Kazakhstan $(0.8 \%),{ }^{6}$ PRC $(0.6 \%)$, Uzbekistan $(0.4 \%)$, Afghanistan $(0.2 \%)$, UAE $(0.3 \%)$, and Turkey $(0.2 \%)$. For Tajikistan, we show that for Tajik migrants the most attractive location for employment in the Russian Federation is Moscow metropolitan area: $46.3 \%$ of all migrants having returned from Russia worked

6 Findings of previous studies show changes in migration trends that took place over the last 5 years. In 1999, migrants from Tajikistan mainly headed for the Russian Federation (where 92.86\% of them worked) and for Kazakhstan (7.14\%). By 2002, the number of migrants who worked in Kazakhstan dropped to 1.3\% (Olimova and Bosk, 2003). This reduction is caused by the waning of economic attraction of going to that country, the increased costs of transportation to Kazakhstan and by competition on the part of labour migrants from Uzbekistan. 
there. The main areas of employment are construction, and, to a lesser degree, trade and services sector (Brown, Olimova and Boboev, 2008).

The Siberian Federal District is the second important host territory for Tajik migrants after Moscow and the Moscow Province. Significantly less attractive for migrants are Ural and Privolzhsky Federal District. Some concentration of Tajik migrants can be observed in Ekaterinburg (4.3\%), Tyumen (2.7\%), Nizhnevartovsk (1.2\%), Surgut $(1.4 \%)$ and other Siberian towns. Here migrants work on oil and gas wells, construction, chemical production and industrial enterprises. Attractive are also such large industrial centres as St. Petersburg (6.4\%), Samara (4.4\%) and Rostov upon Don (2.9\%).

\section{Legal Status}

The legal status of migrants is one of the most important factors determining their behaviour, their income, the migration efficiency both for the migrants themselves and the countries of destination and origin. Although labour migration brings huge economic and social benefits to both countries of origin and destination, serious informal and institutional constraints have been created for labour migrants in the CIS. This has resulted in the violation of the legislation regulating the stay and employment in the destination country by many migrants and has lead to the current situation where irregular migration dominates regular migration.

Tajikistan has a visa-free regime with the Russian Federation, therefore Tajik citizens' travel to Russia within a legal framework. However, there are many irregular migrants among the labour migrants from Tajikistan. Sometimes this stems from irregularities concerning registration in the place of residence: $16.5 \%$ of the sample returnees stayed in the places of settlement illegally, that is to say, they have not been registered in OVIR (Department for the Issuance of Visas and Registration), and 8.6\% reported that they registered occasionally. The most frequent reason behind the lack of registration is that every fourth wage worker living abroad said that he saw no reason for registration, since he had to pay bribes to the police in order to be able to stay in a specific area. The same number of informants noted that the registration fee was expensive and they could not afford it. Nearly $70 \%$ of irregular migrants did not leave their workplace, where they lived at the same time.

According to the behavioural survey of migrants the share of workers with an unregulated status reaches $52 \%$. These studies on trafficking in human beings with regard to Tajikistan reveal that more than $70 \%$ of migrants from Tajikistan working in Russia are prone to various forms of trafficking; in other words their freedom of movement is restricted, frequently, they do not have any documents, these documents have often been withdrawn by employers (Olimova and Kurbanova, 2006).

Migrant women register in the place of their residence more frequently than men: $83.3 \%$ compared to $74.7 \%$ of male migrants. However, a significantly higher percentage of women seldom legalise their labour relations $73.3 \%$ compared to $25.6 \%$ of men who work on the basis of a verbal agreement. 
With the enforcement of the new migration legislation in the Russian Federation in 2007, the number of legal migrants, who live and work on the territory of the Russian Federation, has significantly increased. In 2006, the Federal Migration Service (FMS) registered about 30 thousand migrants from Tajikistan, and already in 2007 the number of legal labour migrants in Russia had reached 200 thousand. Nevertheless, in spite of some degree of liberalisation of the migration regime in the Russian Federation, the problem of irregular migration is still pressing.

\section{Return Plans}

According to Brown, Olimova and Boboev (2008), 88\% migrants, who worked abroad in 2006, planned to work abroad in the future, and $83.9 \%$ stated that they would go to work abroad in 2007. Those who were not certain about their future plans constituted $3.2 \% .8 .8 \%$ of the total number of migrants quite clearly did not intend to work abroad in the future.

\section{THE IMPACT OF MIGRATION ON EDUCATION}

When addressing the educational levels of migrants it is necessary to mention that overall the educational level of migrants has fallen over the past 10 years. The question arises if the drop in the educational level of migrants is related to the deterioration of human capital in Tajikistan or rather to the negative effects of labour migration (such as unregulated legal status of migrants). In response to this question we have made the following hypothesis: Collective experiences during migration cause a decrease in the level of migrants' education throughout the migration development process. Due to an unregulated legal status many migrants in destination countries are faced with conditions in which educational background is becoming irrelevant. This collective experience discourages young prospective migrants to improve their educational level.

To accept or reject this hypothesis we need to understand if a higher level of education can provide any competitive advantage to labour migrants in external labour markets. With this purpose we first have to look at the connection between migrants' educational level and their incomes abroad. If higher educational status is highly correlated with higher incomes abroad, then migrant workers may have strong incentive to acquire skills in Tajikistan despite weak incentives for acquisition of skills within the labour market in Tajikistan.

The data collected on distribution of remittances give some indication of the returns on education for migrant workers although there are many other factors involved in the process aside from the ability to earn higher wages on the basis of higher educational status (Brown et al., 2008). Other influential factors range from the strength of family ties to the weakness of the financial system in Tajikistan. However, variation in the distribution of annual remittances on the basis of the educational status of remitting migrants may still offer some degree of insight into the returns on education associated with employment abroad. 
The graph 1 shows that migrants with professional education remit higher sums of money more frequently than labour migrants with any other level of education and there is a general pattern for individuals with a higher terminal level of education to remit higher sums of money more frequently. The average amount of annual remittances sent by labour migrants in the categories of complete basic, secondary and professional education appear to be quite similar at around USD 501-1500, while those from individuals with incomplete basic education are one category lower at less than USD 500. Thus, acquisition of complete basic education guarantees an ability to earn enough to allow remittances on a par with individuals who have completed secondary and professional education.

\section{Graph 1: Distribution of Annual Remittances by Educational Status of Labour Migrants (in USD) ${ }^{7}$}

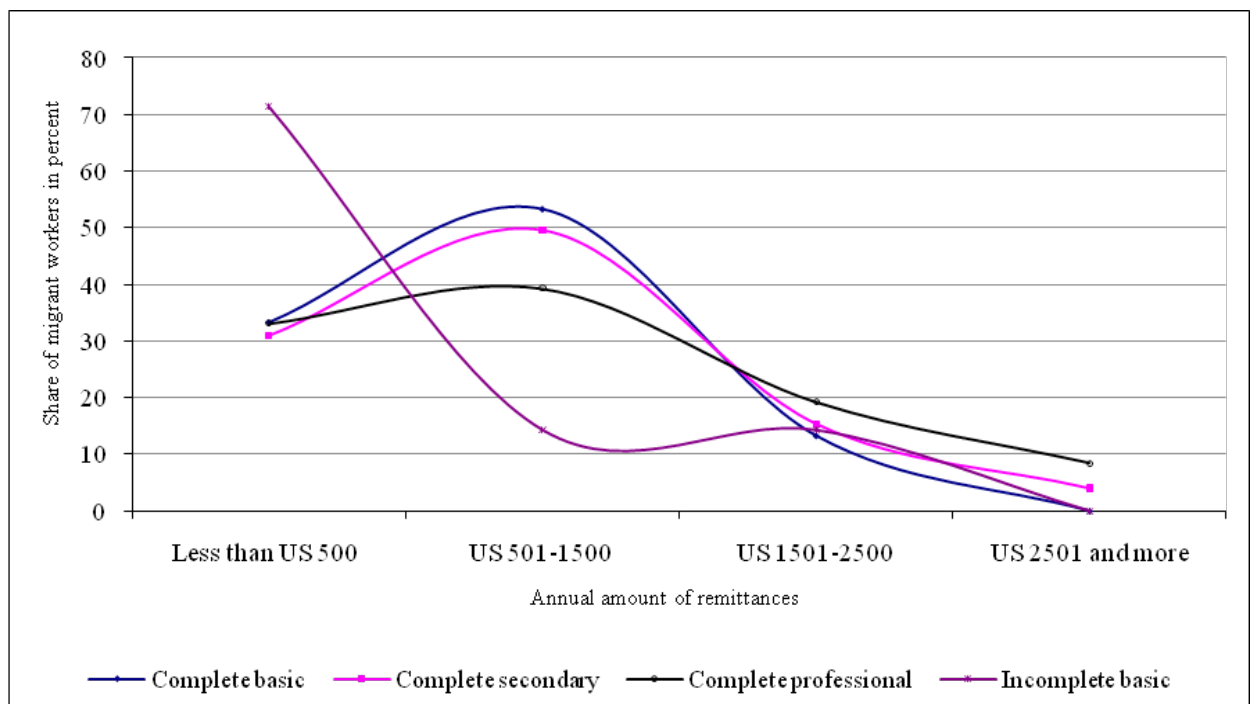

Source: Brown, Olimova and Boboev (2008)

Remittances have a positive impact on poverty alleviation, particularly in rural areas, lowering income inequity and alleviating poverty throughout Tajikistan. Without this income, average poverty incidence would increase by $15 \%$ to $74.5 \%$ of the population, and extreme poverty would climb from $32.2 \%$ to $54.5 \%$ across the country as a whole, and by $25 \%$ in rural areas.

Although labour migration lowers the unemployment rate, workers from Tajikistan are not particularly interested in acquiring professional or technical education at home prior to the move as most of them find work in low skilled sectors such as construc-

7 The category of labour migrants with university education is excluded from the sample due to the very small number of observations (3). 
tion and trade.

Many labour migrants acquire higher skills that improve their productivity and human capital while working abroad. However, very few are given any formal certification of their added capacities so the acquisition of new skills abroad does not easily translate into higher wages.

In 2007, about 700.000 Tajik temporary migrants were working abroad, meaning about $15 \%$ of the economically active population of Tajikistan were labour migrants. More than half a million Tajik people were employed in Russia with significant numbers also in Kazakhstan, the United Arab Emirates and other countries.

Given this scenario, examination of the structure of labour demand and the areas of employment offered in Russia could provide some insight into the incentives for the acquisition of human capital in the form of skills and professional or technical education in Tajikistan.

Brown, Olimova and Boboev (2008) find that $74.1 \%$ of migrants in Russia are employed in construction, $10.8 \%$ in wholesale and trade, $5.4 \%$ in agriculture, $4.8 \%$ in industrial manufacturing and $4.8 \%$ in high skilled services such as education and health care.

The predominant majority of Tajik workers in Russia is employed in industries requiring no or low skills such as construction labour and petty trade, while very few are employed in mid-level skilled areas such as industrial production or higher skilled sectors like health care and education. In this situation, there is little incentive to acquire professional or technical skills beyond the basic literacy level.

However, some labour migrants do increase their human capital by acquiring additional skills and qualifications during employment abroad. The table 5 from Brown, Olimova and Boboev (2008) shows that some labour migrants acquire additional skills they could not learn at home and many workers attempt to acquire professional or technical qualifications abroad by enrolling at local vocational schools or attending short courses, although few succeed in obtaining professional or technical qualifications.

These figures show that $66.1 \%$ of individuals gained proficiency in Russian, which is unsurprising given that the main barrier to increasing the professional level of migrants is the lack of knowledge of Russian. Other obstacles are the absence of specialised programmes for training potential migrants (part-time classes, on-site training, etc.) and mechanisms for funding the education of migrants (educational loans, financial help) in the reception countries. 
Table 5: Additional Qualifications Acquired Abroad

\begin{tabular}{|l|c|c|}
\hline & $\begin{array}{c}\text { Number of } \\
\text { respondents }\end{array}$ & Percent \\
\hline Did not acquire any new qualification & 371 & 58.9 \\
\hline Learned Russian & 416 & 66.1 \\
\hline Learned English & 12 & 1.9 \\
\hline $\begin{array}{l}\text { Acquired new profession and obtained } \\
\text { certificate without examination }\end{array}$ & 11 & 1.7 \\
\hline $\begin{array}{l}\text { Acquired new profession and obtained } \\
\text { certificate after passing an examination }\end{array}$ & 26 & 4.1 \\
\hline Acquired new skills without certificate & 435 & 69.2 \\
\hline Graduated from vocational school abroad & 13 & 2.1 \\
\hline Graduated from university/college abroad & 2 & 0.3 \\
\hline Obtained graduate degree abroad & 4 & 0.6 \\
\hline No response & 9 & 1.4 \\
\hline
\end{tabular}

Source: Brown, Olimova and Boboev (2008) (more than one answer is possible)

\section{Remittances and Education}

The share of expenditures on education and health care in the total structure of household expenditures is small (Table 6). Generally, across the country, the share of expenditures on health care and education in total household expenditures constitutes $3.78 \%$. Data from Brown, Olimova and Boboev (2008) show that the type of residential area and the level of well-being have almost no influence on the share of expenditures on education and health. We can distinguish a slight variation in the share of expenditures on education and health, depending on the level of well-being. Households receiving remittances in the poor quintiles $(1,2$, and 3$)$ spend a little more on education and health than households without remittances do because in Tajikistan these services are not very differentiated in terms of quality and costs. Members of poor and wealthy households consume the same range of medical services in terms of quality. Similarly, the cost of attendance of schools and universities is the same for households of all levels of income.

In general, Tajik households do not make investments in education or health care. Before investing in education, household members need to have a certain degree of confidence about good employment opportunities after education is obtained to cover for the expenditures incurred during their studies. We can attest that members of households in Tajikistan lack this sort of confidence, and consequently, do not heavily invest in education.

The experience of the first decade of labour migration from Tajikistan demonstrates that professional skills of migrants in recipient countries are not in high demand. Since the opportunities for employment even for highly educated and professional indivi- 
duals are scarce, households do not spend resources on education for children.

In addition, the expenditures of education for girls are even less than on boys. Those households, which make investment in education, prefer to provide education mainly for boys, because they hope that educated males will be able to obtain jobs in Tajikistan or elsewhere abroad, thus helping households to procure support when they are old. The investment in education for girls is viewed as bringing no benefit, since girls are expected to leave households at early ages as wives and not to compete in the labour market.

Table 6: Share of Expenditures on Health Care and Education in Total Expenditure (in \%, average for the households in the category)

\begin{tabular}{|l|c|c|c|}
\hline & $\begin{array}{c}\text { All sample } \\
\text { households } \\
(\mathrm{N}=3,300)\end{array}$ & $\begin{array}{c}\text { Receiving } \\
\text { remittances } \\
(\mathrm{N}=1,320)\end{array}$ & $\begin{array}{c}\text { Not receiving } \\
\text { remittances } \\
(\mathrm{N}=1,980)\end{array}$ \\
\hline Total for Tajikistan & 3.8 & 3.7 & 3.9 \\
Dushanbe & 3.4 & 3.1 & 3.5 \\
Other urban areas & 4.3 & 4.0 & 4.5 \\
Rural areas & 3.7 & 3.6 & 3.8 \\
\hline Quintiles based on household income including remittances \\
Quintile 1 & 5.2 & 6.9 & 4.7 \\
Quintile 2 & 4.8 & 4.6 & 4.9 \\
Quintile 3 & 3.9 & 4.1 & 3.8 \\
Quintile 4 & 3.7 & 3.7 & 3.6 \\
Quintile 5 & 2.7 & 2.6 & 2.8 \\
\hline
\end{tabular}

Source: Brown, Olimova and Boboev (2008)

\section{Role of Returning Migrant Workers (Returnees) in Development of Human Capital}

The data obtained from Brown, Olimova and Boboev (2008) show that $9 \%$ of migrants return from abroad to find employment in Tajikistan each year. The basic reasons for returning are age, poor health, family problems and deportation. Working experience and the knowledge acquired abroad offer improved employment prospects for many returnees in Tajikistan. A study of returnees by Olimova (2010) for the International Labour Organization has shown that the experience of employment abroad helped $39.1 \%$ of returnees to find a well paid job in Tajikistan. However, $42.7 \%$ of returnees found this was not the case and $18.2 \%$ found they actually had worse prospects. The same study by the IOM revealed that a total $68.1 \%$ of returnees were employed. Most of the employed returnees preferred to be self employed, running their own business $(21.7 \%)$ or work as independent contract workers (17.3\%). A smaller share of returnees (14.4\%) worked as hired workers in state or publicly owned enterprises and $10.1 \%$ were employed in private 
enterprises. Of the $31.9 \%$ of returnees without jobs, almost $20 \%$ were classed as unemployed; $15.6 \%$ were unemployed and were looking for work and $4.3 \%$ were unemployed and not looking for work.

Our study on returnees (Olimova, 2010: 37) showed that returning migrants actively participate in business development in Tajikistan, but their overall influence on technological development is insignificant.

Returnees play an important role in the modernisation of some enterprises, introducing new technologies and adopting new tools and skills. Meanwhile, work experience from employment abroad is only applied marginally, mostly in small businesses owned and run by the returnees themselves. Hence the greatest impact of adaptation through new techniques, tools and methods introduced by returnees is limited to areas with the highest concentration of small businesses such as services, construction, and manufacturing and the processing and storage of agricultural products. Employers in all these areas prefer to hire returnees and pay them higher salaries.

However, returnees do not have a significant influence on the modernisation of technological process in large state enterprises or in enterprises that undergo extensive and sizeable investment. Also, many enterprises that do not require technological or organisational innovation refuse to hire migrants, meaning that the valuable experience and additional qualification of returnees is not made use of precisely in those areas suffering the most from the lack of qualified workers. The poor health and advanced age of many returnees also form barriers to employment.

If the migrant worker had already been employed before migrating, the departure interrupts his/her career. This problem is aggravated as migrants seldom work in the profession they were trained for, and as a rule, they lose their skills in a certified profession as a consequence. In addition, during their absence the returnees lose the social capital they possessed in Tajikistan and do not accumulate a new one - useful acquaintances, necessary contacts, which would help them to find a decent job in their homeland.

If a migrant formerly worked on a responsible and profitable position, of course after his return he will not be able to occupy this job, as it has been occupied by someone else (interview with migrant Salim, teacher, 45 years old, from Bokhtar,

Tajikistan).

Starting work for a migrant after return from Russia is very difficult. Because, influential acquaintances or big money are required to get a good job or start one's own business (interview with migrant Homidjon, welder, 42 years old, from Isfara,

Tajikistan).

Frequently, the returned migrants have no possibility to use their new experience and skills, they acquired abroad, because of the technological lag in their homeland.

Many migrants fail to find a job after their return, as here the job they were doing abroad does not exist. They are used to working with new technologies. Introducing the technologies, which are being used in Russia, requires much time, money and efforts (interview with migrant Abdunabi, businessman, 30 years old, from Isfara, Tajikistan). 
Nevertheless, even in adverse circumstances the returnees can make use of the skills and of the life experience they have gained in migration in order to organise a profitable activity.

I am a returned migrant, but do not want to get into employment, because my education is not sufficient to find a place with a good salary and it is not profitable to become an ordinary worker - the salary is not enough to sustain the family. Therefore, currently I am working as a taxi driver with my own car (interview with migrant Yokubkhoja, taxi driver, 27 years old, from Isfara, Tajikistan).

Returnees can face problems when their employment abroad is not counted toward their work experience and the period of employment abroad may interrupt their career growth back home. Many migrants will work in different areas of specialisation while they are away and the skills and qualifications they acquired prior to departure may become obsolete. Another common problem for returnees is the lack of opportunity to employ their superior qualifications and experience from employment abroad because of the outdated technology of the respective work sector in Tajikistan.

\section{CONCLUSION}

We find that labour migration has strong effect on the development of human capital of Tajikistan, as $15 \%$ of the population work abroad. Labour migration has both negative and positive effects on education. Migrants with higher education levels tend to send money home more often than migrants with lower levels of education, but, as migrants rarely work in their areas of specialization, the skills and qualifications acquired prior to departure tend to become outdated. However, returnees make a significant contribution to small and medium businesses and agriculture in Tajikistan, bringing new technology, mechanisms, instruments, knowledge and skills. This way, migration serves as a transmitting mechanism for dissemination and adoption of new technology, thereby raising the level of human capital.

Although the education system in Tajikistan prepares individuals poorly for employment abroad and there is no system of professional or technical education for potential migrants, it still provides potential migrants with basic skills such as writing and reading. In return labour migration allows individuals with basic skills to obtain new advanced skills and qualifications. However, as only a very small proportion of individuals have their qualifications certified, the acquisition of new skills abroad does not easily translate into higher wages. In addition, the employment structure for Tajik migrants in Russia encourages labourers to invest in only the minimal level of human capital sufficient for employment in sectors such as construction and trade. The low return on education resulted in a weak demand for educational services and a drop in long-term accumulation of human capital.

The greatest positive effect of migration is the availability of additional resources to spend on education in those families receiving remittances. Children of migrant families have stronger incentives to study foreign languages, have better access to technical equipment and their parents usually have wider horizons, however these households also 
have a higher level of school absenteeism as children are subject to less parental control and are more likely to have to work.

In total the general trends in the post-Soviet transit period resulted in the lower level of education of young people in comparison to their parents, significant drop in the quality of education and a higher inequity in access to education for certain social groups.

\section{References}

ACOSTA Pablo (2006) Labour Supply, School Attendance, and Remittances from International Migration: The Case of El Salvador, World Bank Policy Research, Working Paper No. 3903.

BROWN Richard, OLIMOVA Saodat and BOBOEV Mukhammady (2008) Country Report on Remittances of International Migrants in Tajikistan, in Study on International Migrants' Remittances in Central Asia and South Caucasus, Asian Development Bank, http://www.adb. org/Documents/Reports/Consultant/40038-REG/40038-04-REG-TACR.pdf.

COX EDWARDS Alejandra and URETA Manuelita (2003) International Migration, Remittances and Schooling: Evidence from El Salvador, Journal of Development Economics, 72 (3), pp. 429-461.

DE HAAN Arjan (2005) Migration in the Development Studies Literature: Has it Come out of Marginality?, Paper for UNU-WIDER, Jubilee Conference Future of Development Economics, Department for International Development, United Nations University.

HANSON Gordon and WOODRUFF Christopher (2003) Emigration and Educational Attainment in Mexico, Mimeo, University of California, San Diego.

MCKENZIE David and RAPOPORT Hillel (2006) Can Migration Reduce Educational Attainment? Evidence from Mexico, World Bank Policy Research, Working Paper No. 3952.

OLIMOV Jafar (2007) UNDP Human Development Report: Informal Economy of Tajikistan, Dushanbe, United Nations Development Programme.

OLIMOVA Saodat (2010) Migration and Development in Tajikistan. Emigration, Return and Diaspora (draft), International Labour Organization, Dushanbe.

OLIMOVA Saodat and BOSC Igor (2003) Labour migration from Tajikistan, International Organization for Migration, http://www.iom.int/jahia/webdav/site/myjahiasite/shared/shared/ mainsite/published_docs/studies_and_reports/Tajik_study_oct_03.pdf [16 July 2009].

OLIMOVA Saodat and KURBANOVA Rukhshona (2006) Behavioral Research among Migrant of Tajikistan on HIV and STD, International Organization for Migration, draft, www.ilo.org/ wcmsp5/groups/public/---ed.../wcms_120534.pdf.

QUDDUSOV Jamshed (2009) Labour Market Review in Tajikistan, Working paper.

Republic of Tajikistan (2006) Tajikistan in Figures 2006. Statistical Pocketbook, Dushanbe, State Committee on Statistics of the Republic of Tajikistan.

SCSRT (2006) 15 years of Independence. Statistical Report of Tajikistan 2006, State Statistical Committee of Tajikistan, Dushanbe, 2006.

TLSS (2007) Tajik Living Standards Survey 2007, State Statistical Committee of Tajikistan, Dushanbe.

THIEME Susan (2009) Where to Return to? Rural Urban Interlinkages in Times of Internal and International Migration in Social Research Center, Amercian University Central Asia (AUCA), Policy Briefs on Civil Society. Migration, Islam, and Corruption, Bishkek, AUCA, pp. 108-113.

UNDP (2005) Central Asia Human Development Report, United Nations Development Programme, Bratislava, http://europeandcis.undp.org/poverty/show/300BDC00-F203-1EE9BE944F24EDFC09CE [16 July 2009].

World Bank (2005) Republic of Tajikistan Poverty Assessment Update, World Bank Report, No. 30853-TJ. 


\title{
Revue européenne des migrations internationales
}

vol. $26-\mathrm{n}^{\circ} 3$ (2010)

Migrations en Asie centrale et au Caucase

Marc-Antoine Pérouse de Montclos

\section{Réfugié ou déplacé ? Les enjeux d'une requalification : l'exemple de la Géorgie après la guerre de 2008}

\author{
Avertissement \\ Le contenu de ce site relève de la législation française sur la propriété intellectuelle et est la propriété exclusive de \\ l'éditeur. \\ Les œuvres figurant sur ce site peuvent être consultées et reproduites sur un support papier ou numérique sous \\ réserve qu'elles soient strictement réservées à un usage soit personnel, soit scientifique ou pédagogique excluant \\ toute exploitation commerciale. La reproduction devra obligatoirement mentionner l'éditeur, le nom de la revue, \\ l'auteur et la référence du document. \\ Toute autre reproduction est interdite sauf accord préalable de l'éditeur, en dehors des cas prévus par la législation \\ en vigueur en France.
}

\section{revues.org}

Revues.org est un portail de revues en sciences humaines et sociales développé par le Cléo, Centre pour l'édition électronique ouverte (CNRS, EHESS, UP, UAPV).

\section{Référence électronique}

Marc-Antoine Pérouse de Montclos, « Réfugié ou déplacé ? Les enjeux d'une requalification : I'exemple de la Géorgie après la guerre de 2008 », Revue européenne des migrations internationales [En ligne], vol. 26 - $n^{\circ} 3$ | 2010, mis en ligne le 01 décembre 2013, consulté le 23 janvier 2014. URL : http://remi.revues.org/5240 ; DOI : 10.4000/ remi. 5240

\section{Éditeur : Université de Poitiers}

http://remi.revues.org

http://www.revues.org

Document accessible en ligne sur : http://remi.revues.org/5240

Ce document est le fac-similé de l'édition papier.

(c) Université de Poitiers 


\section{Note de recherche}

\section{Réfugié ou déplacé ? Les enjeux d'une requalification : l'exemple de la Géorgie après la guerre de 2008}

\section{Marc-Antoine PÉROUSE DE MONTCLOS ${ }^{1}$}

$\mathrm{D}^{2}$ ans l'entendement populaire, le réfugié est d'abord et avant tout quelqu'un qui fuit un danger, quoi qu'il en soit par ailleurs de la nature des persécutions subies, des distances parcourues et du franchissement ou non d'une frontière internationale. Sur le plan pratique, cependant, l'attribution d'un statut de demandeur d'asile s'avère déterminante pour les migrants et les États de départ ou d'accueil. En effet, la qualification juridique des populations chassées de leurs foyers par des conflits armés a d'importantes implications politiques selon que les victimes sont considérées comme des déplacés internes ou des réfugiés qui ont franchi une frontière internationale et qui, à ce titre, peuvent bénéficier du dispositif de protection de la Convention de Genève de 1951. Partant de l'exemple de la Géorgie après la guerre qui l'a brièvement opposée à la Russie en 2008, cet article vise ainsi à analyser les enjeux stratégiques que révèle la réinstallation de ressortissants de facto traités en réfugiés à la suite de la proclamation d'indépendance des territoires sécessionnistes d'Ossétie du Sud et d'Abkhazie². Du Haut Karabakh à la Tchétchénie, le Caucase est, on le sait, une région ravagée par des conflits à répétition qui ont provoqué de nombreux flux migratoires. Conjugués aux crises économiques structurelles des espaces post-soviétiques, les déplacements causés par des troubles politiques s'y sont en l'occurrence ajoutés à un exode déjà massif de femmes et d'hommes partis chercher du travail et un avenir meilleur à l'étranger. La Géorgie, en particulier, s'est progressivement vidée d'une partie de ses habitants. Depuis l'indépendance en 1991, elle a perdu environ un cinquième de sa population et la défaite de 2008 a sans doute précipité le mouvement en mettant un terme aux espoirs qu'avait soulevés la Révolution des Roses après l'effondrement du régime mafieux d'Édouard Chevardnadze en 2003 (Kleinhanss,

1 Docteur en sciences politiques, Chargé de recherche à l'Institut de Recherche pour le Développement, CEPED (Centre Population et Développement), UMR196, Université Paris Descartes, INED, IRD, 19, rue Jacob, 75006 Paris ; marc-antoine.perouse@ird.fr

2 Cet article est tiré d'une mission de terrain réalisée en Géorgie en octobre 2008. Je tiens particulièrement à remercier Éveline Baumann, de l'IRD (Bondy), Marina Muskhelishvili, du Centre for Social Studies (Tbilissi) et les deux lecteurs anonymes qui m'ont permis d'affiner l'analyse. 
2008 ; Serrano, 2007, 2008 ; Shelley et al., 2007 ; Stefes, 2006 ; Zourabichvili, 2009).

Dans le Caucase, la singularité de la Géorgie tient aussi à la façon dont le gouvernement de Mikheil Saakachvili a géré l'afflux des victimes déplacées par le conflit d'août 2008. En réinstallant définitivement les « réfugiés » géorgiens qui avaient fui l'Ossétie du Sud et l'Abkhazie, les autorités ont en effet contribué à valider un statu quo qui revenait à reconnaître implicitement l'existence de frontières internationales autour des régions sécessionnistes. De ce point de vue, la situation paraît très différente de celle des pays voisins où l'on a plutôt cherché à renvoyer les déplacés près des zones de combat afin de maintenir une pression humaine sur des territoires perdus ou conquis. Le gouvernement d'Erevan, par exemple, a facilité le retour des Arméniens originaires du Haut-Karabakh, d'Abkhazie et de Chahoumian. Créé pour l'occasion en novembre 1991, son comité d'État pour les « réfugiés » s'est seulement occupé d'intégrer les Arméniens originaires d'Azerbaïdjan, à qui il a donné un petit pécule, des terres, le droit de vote et la nationalité du pays d'accueil. À Bakou, une loi de septembre 1998 a pareillement accordé la citoyenneté du pays d'accueil aux Azéris d'Arménie, aux Meskhètes venus d'Ouzbékistan et aux Iraniens qui avaient déjà obtenu un statut d'exilé pendant la période soviétique. En revanche, les autorités ont refusé de réinstaller les déplacés azéris massés dans les régions frontalières du Haut-Karabakh après son annexion militaire par Erevan en mai 1994. Au contraire, le gouvernement a tout fait pour entretenir une sorte de bouclier humain qui devait exprimer son intention de reconquérir les territoires perdus. Il a fallu attendre cinq bonnes années avant que, pour la première fois, le président Heydar Aliev commence à envisager officiellement de réinstaller les déplacés du Haut-Karabakh, qui vivaient dans des conditions très précaires et dont le mécontentement allait grandissant (MacFarlane, 2000 ; Kevonian, 2000).

\section{LES MIGRATIONS FORCÉES EN GÉORGIE : UNE PERSPEC- TIVE INSTITUTIONNELLE}

Par contraste, le gouvernement géorgien, lui, a réagi très vite après les combats d'août 2008. D'un régime à l'autre, sa position a beaucoup évolué en la matière. Initialement, Tbilissi n'avait pas vraiment cherché à distinguer les "réfugiés » des " déplacés » pour qualifier le statut des victimes des conflits sécessionnistes qui avaient contribué à démembrer le territoire national depuis le début des années 1990. Après avoir adhéré à la Convention de Genève de 1951, le gouvernement avait ainsi créé en 1999 un ministère des « Réfugiés » pour gérer les déplacés internes comme les demandeurs d'asile en provenance, notamment, de Tchétchénie. Ce faisant, la Géorgie se dotait simplement des outils juridiques et institutionnels dont disposaient déjà les autres pays de la région. Dès septembre 1992, par exemple, l'Azerbaïdjan avait adopté une loi sur les réfugiés et les personnes déplacées. L'Arménie, pour sa part, avait établi un département des migrations et des réfugiés dans le cadre d'une loi votée en mars 1999 à la suite d'une Conférence organisée à Genève en mai 1996 sous l'égide du HCR (Haut Commissariat des Nations Unies pour les réfugiés), en l'occurrence dans une perspective transcaucasienne, avec la Russie et les autres membres de la CEI (Communauté des États indépendants). 
Dans le cas de la Géorgie, les autorités se sont surtout préoccupées de définir les catégories de migrants lorsque la crise d'août 2008 a fini par entériner la sécession de territoires autonomes devenus de facto indépendants. L'État a alors désigné tous les sinistrés sous le vocable plus neutre de "sans abris ». La nouvelle terminologie officielle lui a permis d'évacuer deux catégories potentiellement dangereuses sur le plan politique : celle de « réfugié », qui aurait impliqué le passage d'une frontière internationale et, partant, une reconnaissance informelle des indépendances ossète et abkhaze ; et celle de " déplacé », une notion qui renvoie au passé post-communiste des victimes des conflits de 1991-1993, avant la Révolution des Roses et l'arrivée au pouvoir du président Mikheil Saakachvili en 2004. En pratique, l'État géorgien a ainsi essayé de dépasser les limites juridiques qui auraient pu restreindre l'aide occidentale. Malgré de nombreux dysfonctionnements administratifs, il a notamment entrepris de réinstaller très rapidement les « sans abris » de la guerre de 2008, comme pour mieux effacer les traces de la défaite. Le relogement définitif de ces populations, qui revenait à accepter le fait accompli, a certes semblé surprenant de la part d'un gouvernement nationaliste qui proclamait ouvertement sa volonté de récupérer les territoires perdus. Mais l'empressement des autorités a témoigné d'enjeux économiques et sécuritaires qui visaient en priorité à stabiliser le régime du président Mikheil Saakachvili et à mettre les victimes de la guerre à l'abri des foyers de tension en Abkhazie et en Ossétie du Sud. En filigrane, la politique de relogement du gouvernement a aussi révélé des ambitions à plus long terme en vue de repeupler des régions susceptibles de relancer l'économie nationale.

De fait, les combats d'août 2008, aussi brefs aient-ils été, ont eu un impact important. Les destructions commises par les troupes russes et les milices ossètes ont en effet touché une région ravagée en profondeur par les réformes libérales de l'ère postsoviétique. Frontalière de la zone de combats, la province de Shida Kartli en témoigne à sa manière ${ }^{3}$. Selon une enquête de la Banque Mondiale réalisée en octobre 2007, elle était déjà la moins bien lotie du pays, avec une proportion de pauvres deux fois supérieure à la moyenne nationale, à raison de $59 \%$ contre $33 \%$ (World Bank, 2008). Là-dessus, le conflit est venu achever de ruiner les petits cultivateurs de la région. Outre la perte des récoltes, quelque 40000 familles sont désormais confrontées à de sérieux problèmes d'irrigation depuis qu'à Tskhinvali, le pouvoir ossète a fermé les vannes de la rivière Liakhvi en juillet 2008. Dans un pays où le salariat ne concerne qu'un tiers des Géorgiens actifs, la crise a ainsi aggravé la vulnérabilité d'une population rurale qui, d'habitude, consomme une bonne partie de sa production agricole et vend le reste sur les marchés à la marge de l'économie formelle (Baumann, 2009).

Surtout, l'exode qu'a provoqué la guerre s'est greffé sur de précédentes vagues de déplacements liées aux conflits des années 1991-1993, alourdissant d'autant la charge de l'assistance sociale dans le budget de l'État. Les combats d'août 2008 et l'avancée

3 Sur les 120 maisons que comptait le village d'Ergneti, par exemple, seulement dix-huit ont été épargnées par les combattants qui ont pillé les lieux. Situé sur la ligne de front dans la banlieue de Tskhinvali, la capitale de l'Ossétie du Sud, l'endroit est symbolique car il a pendant longtemps été un important centre de contrebande et un des derniers points de contact et de commerce avec les sécessionnistes avant le démantèlement de son marché par les autorités géorgiennes en 2004. Les villages alentour n'ont pas non plus échappé à la fureur des milices ossètes, qui s'est propagée jusqu'à la ville voisine de Gori, le chef-lieu administratif de la province de Shida Kartli. 
des troupes russes ont chassé de leurs foyers près de 128000 personnes en Géorgie. À la fin octobre 2008, plus de 42000 d'entre elles n'avaient toujours pas pu rentrer chez elles et continuaient de vivre dans des centres collectifs. Comme d'habitude en période de crise, il convient certes de manier les chiffres avec précaution. On estime que $20 \%$ des déplacés d'août 2008 ont perdu leur carte d'identité au cours de leur fuite. Faute de papiers, certains ont pu être enregistrés à plusieurs reprises dans les centres collectifs, compensant en quelque sorte «l'invisibilité » de leurs camarades d'infortune qui n'ont pas été recensés par les autorités parce qu'ils avaient d'eux-mêmes trouvé à se loger chez des parents à Tbilissi ou dans les environs. Quoi qu'il en soit des incertitudes à ce sujet, leur poids démographique n'a rien de dérisoire à l'échelle d'un pays d'environ 4 millions d'habitants. Il s'avère d'autant plus conséquent qu'il vient s'ajouter aux 264000 déplacés de la « première génération », qui représentaient déjà $6 \%$ de la population géorgienne en 2003.

\section{DES DÉPLACÉS À GÉOGRAPHIE VARIABLE}

Pour bien comprendre les enjeux économiques de la reconstruction, il importe alors de préciser les différents statuts de victimes qui ont déterminé l'approche des autorités en termes d'urgence humanitaire ou de développement. Grosso modo, on peut distinguer trois catégories de déplacés à l'issue de la crise d'août 2008. Les premiers sont originaires de la région de Gori et le gouvernement s'est empressé de les évacuer de Tbilissi pour les réinstaller chez eux dès que les troupes russes ont commencé à se retirer à la fin septembre : nous aurons l'occasion de revenir sur les raisons politiques et économiques d'une telle précipitation. Les seconds, eux, viennent des villages détruits sur la ligne de front le long de l'ancienne frontière administrative soviétique de l'Oblast autonome d'Ossétie du Sud. Malgré les pressions du gouvernement et les efforts des organisations humanitaires en vue de réhabiliter les maisons éventrées avant l'arrivée de l'hiver, ceux-ci ont hésité à revenir chez eux et ont attendu de voir comment la situation militaire allait évoluer. Une troisième catégorie, enfin, comprend ce qu'on pourrait appeler des déplacés « durables », en l'occurrence des Géorgiens qui vivaient en Ossétie du Sud ou en Abkhazie. Parce qu'ils n'ont quasiment aucun espoir de retour, leur intégration s'inscrit dans une perspective à long terme et relève bien d'une politique de développement.

Ces déplacés « durables », il faut le noter, proviennent essentiellement d'Ossétie du Sud. Lors de la crise d'août 2008, il n'y a pas eu de combats en Abkhazie. Les troupes géorgiennes se sont contentées d'abandonner leurs positions dans les gorges de Kodori, incitant quelque 1200 habitants à évacuer la région, peut-être jusqu'à 3000 selon les chiffres du HCR. L'Ossétie du Sud, en revanche, a été beaucoup plus touchée. La Géorgie y a perdu les derniers territoires qu'elle contrôlait encore à l'intérieur de l'ancien Oblast, à savoir les villages de la vallée de Liakhvi au nord de Tskhinvali, où Tbilissi avait installé une administration provisoire dans la localité de Kurta, et le district d'Akhalgori, qui avait été formellement rattaché à une nouvelle province créée pour les besoins de la cause, Mtskheta-Mtianeti. En tout, 16000 personnes auraient été chassées de chez elles : plus, donc, que les 12000 déplacés issus du précédent conflit d'Ossétie du Sud en 1991-1992, sachant que des Géorgiens continuaient de fuir le district d'Akhalgori dans le courant du mois de novembre 2008 . 
$\mathrm{Au}$ vu de l'état actuel des rapports de force, un retour n'est guère envisageable. Alors que le gouvernement Vladimir Poutine a reconnu l'indépendance de l'Ossétie du Sud, le conflit a en effet pris une dimension communautaire qui va retarder d'autant les possibilités de réconciliation. À l'instar des Abkhazes en 1993, les Ossètes ont entrepris en 2008 de mener une politique de nettoyage ethnique afin de se débarrasser définitivement des derniers Géorgiens résidant sur leur territoire. Des années de tension permanente ont fini par avoir raison de la diversité culturelle de la région. Il y a encore vingt ans, la population était beaucoup plus mélangée. D'après les données du recensement de 1989, les Ossètes vivant en Géorgie (164 000) étaient deux à trois fois plus nombreux qu'en Ossétie du Sud (65 000), selon le ministère des Réfugiés (Ministry of Refugees, 2002 : 393). Leur répartition géographique était plus dispersée que celle des Abkhazes, qui étaient à peu près autant $(95000)$ à habiter en Géorgie et dans la république autonome d'Abkhazie. Depuis lors, la situation a radicalement changé. À l'intérieur de la Géorgie, la proportion d'Ossètes est tombée de $3 \%$ de la population en 1989 à $0,9 \%$ en 2002. Parallèlement, la quasi-totalité des derniers Géorgiens d'Ossétie du Sud a été expulsée à la faveur de la guerre de 2008. Quelques Ossètes sont aussi partis vers le sud parce qu'ils étaient accusés d'avoir collaboré avec les autorités de Tbilissi et se sentaient menacés par les indépendantistes.

Dans un tel contexte, les déplacés originaires de la région sont appelés à se réinstaller définitivement en Géorgie. Le gouvernement a en l'occurrence prévu de les reloger avant l'hiver 2008 en réhabilitant 2125 appartements dans des centres collectifs répartis dans les différentes provinces du pays. À cette fin, il a également entrepris de construire 4095 maisons entre Tbilissi et Gori. Le chantier est très symbolique car les nouveaux logements s'égrènent le long de la principale autoroute du pays, un moment occupée par les Russes. Placés sous la responsabilité du ministre de l'Intérieur Vano Merabishvili, qui a utilisé la police pour hâter le mouvement, les travaux ont été réalisés en un temps record, souvent au détriment de la qualité et dans le but évident de démontrer l'efficacité du gouvernement. Sous prétexte de contribuer à la reconstruction nationale, les entrepreneurs ont dû travailler à prix cassés et le chantier n'a donc pas vraiment pu relancer le secteur du bâtiment, qui avait tiré la croissance de l'économie géorgienne ces dernières années. En revanche, les travaux ont permis de convaincre la communauté internationale de financer directement le gouvernement plutôt que les organisations humanitaires. Pris de court, le HCR a littéralement été court-circuité à cet égard, avec un projet de construction plus onéreux et moins bien loti, à raison d'un coût de 20000 dollars par maison et d'une surface de quarante-cinq mètres carrés par famille, contre respectivement 14000 dollars et soixante-cinq mètres carrés du côté du ministère de l'Intérieur.

\section{DES ENJEUX POLITIQUES}

L'enjeu, ici, était bien d'affirmer la prééminence de l'État. De fait, les organisations humanitaires n'ont convoyé qu'une partie de l'assistance apportée aux victimes de la guerre de 2008. Certaines étaient déjà présentes avant les événements, à l'instar de Médecins sans frontières, World Vision ou le Conseil norvégien des réfugiés ; d'autres sont arrivées au moment de la crise, telle Première Urgence. Dans tous les cas, les solidarités de voisinage ont d'abord joué à plein pour secourir les déplacés arrachés à leurs 
foyers. Avec ses œuvres caritatives, l'Église orthodoxe de Géorgie n'a pas non plus été en reste ; elle pourrait d'ailleurs être amenée un jour à assumer une fonction de médiation informelle grâce aux bonnes relations qu'elle entretient avec son homologue russe, qui a refusé d'intégrer les clergés ossète et abkhaze ${ }^{4}$. De plus en plus nombreuse, la diaspora géorgienne a également fourni un apport important. Selon une enquête réalisée en juillet 2007 et commanditée par la BERD (Banque européenne pour la reconstruction et le développement), ses envois de fonds avoisinent les 418 millions de dollars par an, provenant pour moitié des émigrés en Russie et représentant près de $6 \%$ du produit intérieur brut en 2006 (B\&A, 2007). D'après d'autres modes de calcul, ils auraient même atteint jusqu'à 546 millions de dollars en 2006, voire 800 millions de dollars et $10 \%$ du produit intérieur brut si l'on inclut les envois de marchandises et les transferts informels (Kakulia, 2007). De tels chiffres relativisent de beaucoup l'apport de l'aide humanitaire occidentale.

Le gouvernement, quant à lui, n'a pas renoncé à ses prérogatives et s'est empressé, pour des raisons politiques, de renvoyer la majorité des déplacés dans leur région d'origine, autour de Gori. Les centres collectifs de Tbilissi, où s'étaient réfugiées les victimes du conflit, ont en l'occurrence commencé à être vidés avant même que les conditions soient réunies pour assurer un retour décent dans des villages sécurisés où les paysans pourraient reprendre possession de leur maison, bénéficier d'une assistance humanitaire et cultiver leurs champs sans risquer de sauter sur une mine. L'histoire du camp de Gori est significative. Sur le terrain d'un parc municipal qui a accueilli jusqu'à 2400 personnes, les autorités ont en effet planté des tentes pour recevoir des déplacés qui avaient déjà trouvé un toit où s'abriter à Tbilissi. Les premiers convois ont été organisés dès le 23 août, un jour à peine après le départ de Gori des forces d'occupation russes. Un millier de personnes ont bientôt été débarquées dans un terrain vague sans toilettes ni eau chaude pour se laver, seulement des repas froids et inadaptés aux besoins des enfants en bas âge. Après qu'un homme et une femme ont été tués par l'explosion d'une mine dans les environs, il a fallu l'intervention d'un médiateur officiel, l'ombudsman, pour que les autorités suspendent les transferts le 29 août et laissent au HCR et à la Croix-Rouge italienne le soin de préparer convenablement le terrain à partir du 4 septembre. La pression ne s'est pas relâchée pour autant. Pour des raisons de visibilité médiatique, les humanitaires occidentaux ont concentré leurs efforts sur la région de Gori et délaissé Tbilissi alors que la majorité des déplacés continuait de se masser dans la capitale. Les autorités, pour leur part, ont parfois menacé de couper les vivres des centres collectifs afin d'inciter leurs occupants à rentrer chez eux. Début octobre, enfin, le camp de Gori était démantelé et le ministère des Réfugiés publiait une liste de cinquante villages " sûrs » où les habitants étaient censés revenir avec l'aide des humanitaires occidentaux.

Officiellement, l'empressement du gouvernement était justifié par la nécessité de rouvrir les écoles occupées par les déplacés au moment de la rentrée des classes de septembre. En arrière-plan, la précipitation des autorités a également répondu à des consi-

4 Récemment décédé, le patriarche de Moscou, Alexis II, était ainsi très proche de son collègue géorgien Ilya II, qu'il connaissait depuis l'époque soviétique et qui partageait ses vues contre le prosélytisme protestant occidental. En août 2008, il a refusé d'avaliser les modifications de frontières imposées par le pouvoir séculaire : à ses yeux, un tel précédent aurait présenté l'inconvénient de donner des arguments aux clergés sécessionnistes de l'Église orthodoxe en Ukraine, en Moldavie et en Estonie. 
dérations d'ordre stratégique. Il s'agissait d'abord d'occuper le terrain après le retrait des Russes. Ensuite, l'idée était de disperser en province des déplacés susceptibles de rejoindre l'opposition dans la capitale, les campagnes étant davantage acquises au président Mikheil Saakashvili. Enfin, l'objectif était d'effacer les traces de la défaite. Initialement, les autorités avaient cherché à mettre en évidence les victimes de l'occupation russe, regroupées dans des camps de tentes à Gori ou à proximité de l'aéroport de Tbilissi afin de sensibiliser la communauté internationale. Mais une fois les troupes de Moscou repliées en Ossétie du Sud, le gouvernement a plutôt essayé d'occulter un problème qui rappelait crûment les conséquences humaines de son échec militaire. Faute de médias indépendants, des journalistes aux ordres du pouvoir ont bientôt reçu pour instruction d'insister sur les pertes ennemies et de ne plus évoquer le sort des déplacés, sans même parler des soldats géorgiens morts au combat, enterrés à la sauvette et rarement nommés individuellement.

\section{UN POTENTIEL DÉSTABILISATEUR}

Le potentiel déstabilisateur des populations directement victimes du conflit reste en fait la grande inconnue politique du moment. En Géorgie, deux thèses s'affrontent à ce propos. Selon la première, le président Mikheil Saakashvili va tenter de fidéliser les déplacés d'Ossétie du Sud. Avant de fuir leurs villages, une partie d'entre eux avait déjà bénéficié d'un traitement de faveur afin de les inciter à rester autour de Kurta, la capitale provisoire du gouvernement provincial mis en place par Tbilissi au nord de Tskhinvali. Pour leur part, les déplacés de la première génération n'étaient pas restés insensibles à la rhétorique nationaliste d'un président qui promettait de reconquérir les territoires perdus. Aujourd'hui, il se pourrait donc que les autorités essaient de transformer cette population en clientèle électorale à un moment où la cote de popularité du gouvernement ne cesse de baisser. Une telle démarche n'est certes pas un gage de stabilité politique. Bien qu'il n'ait pas intérêt à provoquer les Russes, le président Mikheil Saakashvili est régulièrement soupçonné de vouloir entretenir la crise pour obliger les Occidentaux à le soutenir en passant sous silence la militarisation et la dérive autoritaire de son régime. Le risque serait alors que les déplacés d'Ossétie soient instrumentalisés afin de continuer la guerre par procuration. Il y a eu des précédents en Abkhazie, même si le régime d'Édouard Chevardnadze n'a jamais voulu armer et financer les Mingréliens de la région, dont le pouvoir se méfiait parce qu'ils avaient soutenu l'opposition nationaliste ralliée au premier président du pays à l'indépendance en 1991, Zviad Gamsakhourdia.

Selon une autre thèse, les déplacés de 2008 ne présenteraient en réalité aucun danger de la sorte. En témoignent l'apathie et la faible mobilisation de leurs prédécesseurs chassés d'Ossétie du Sud à la suite des conflits de 1991-1992. Contrairement à leurs collègues d'infortune en provenance d'Abkhazie, qui étaient plus nombreux et mieux organisés, ces déplacés de la première génération ont été particulièrement peu influents et n'ont pas réussi à monter de véritable lobby au Parlement à Tbilissi. D'après des sondages, seulement $3 \%$ disaient appartenir à un parti politique, un groupe social ou une communauté religieuse (Sumbadze et Tarkhan-Mouravi, 2003 : 33-35). Il est vrai que leur statut les a pendant longtemps relégués au rang de citoyens de seconde zone, sans droit de vote puisque leur circonscription n'était plus sous le contrôle de Tbilissi. Il a fallu attendre avril 2002 pour que le code électoral soit modifié et les autorise à voter aux législatives dans 
la circonscription où ils résidaient. Depuis lors, les déplacés d'Ossétie du Sud peuvent également se rendre aux urnes pour les municipales, mais toujours pas être élus.

À présent, leur potentiel déstabilisateur tiendrait plutôt au fait qu'ils sont susceptibles de basculer dans l'opposition, voire de catalyser les mécontentements. En effet, le président Mikheil Saakashvili a beaucoup perdu de la popularité qui l'avait amené au pouvoir suite à la Révolution des Roses en 2003. Son aventure militaire en Ossétie a été un désastre pour les nationalistes et s'est conclue par la perte des derniers territoires restés sous le contrôle de Tbilissi à l'intérieur de la république sécessionniste, en l'occurrence le district d'Akhalgori et les villages autour de Kurta. Autre motif de mécontentement, les déplacés n'ont guère été épargnés par les combats. Alors que Moscou prenait soin de faire évacuer les femmes et les enfants de Tskhinvali dès le 4 août 2008, les autorités de Tbilissi n'ont rien entrepris de la sorte et ont laissé les villageois de Kurta pris en otages sous un déluge de feu. Que l'on retienne la thèse de l'agression russe ou géorgienne importe peu. Dans tous les cas, la montée des tensions aurait dû pousser le gouvernement de Tbilissi à organiser la protection des civils avant le déclenchement du conflit dans la nuit du 7 au 8 août. Certains y voient la preuve d'un évident cynisme. De même que l'évacuation préventive des familles ossètes de Tskhinvali a été présentée comme la confirmation de l'existence d'un piège tendu par les Russes, le maintien de la population géorgienne dans la région de Kurta devait peut-être servir à mettre en place une administration civile aussitôt après l'occupation du territoire sécessionniste par les troupes de Tbilissi ${ }^{5}$.

\section{DES TENSIONS SOCIALES}

Plus important encore pour la stabilité du pays, l'arrivée d'une nouvelle vague de déplacés a ravivé des tensions sociales sous-jacentes depuis une dizaine d'années. Les différences de traitement sont en effet flagrantes. Autant le gouvernement de Mikheil Saakashvili a très vite mis en œuvre une politique visant à accompagner et réinstaller les nouveaux arrivants à l'intérieur de la Géorgie, autant les déplacés de la première génération ont des raisons de se plaindre d'avoir été abandonnés à leur triste sort par le précédent régime, qui a laissé la situation pourrir. À la tête d'un État corrompu et sans ressources budgétaires, le président Édouard Chevardnadze n'avait certes pas les moyens d'assister les victimes des conflits de 1991-1993. Mais plus fondamentalement, les autorités de l'époque ne souhaitaient pas intégrer des populations qu'une réinstallation définitive en Géorgie aurait dissuadées de revenir dans leur région natale, au risque d'entériner la sécession des territoires perdus en Abkhazie et en Ossétie. Pour sauver les apparences, les déplacés d'Abkhazie, par exemple, ont été gérés par une administration provinciale en exil à Tbilissi. Pendant longtemps, le pouvoir central, lui, n'a pas voulu les prendre en charge. Au niveau national, seuls quelques décrets ont traité du problème de façon $a d$ hoc et parcellaire avant l'adoption d'une loi qui, en juin 1996, a pour la première fois officialisé le principe d'une assistance sociale aux déplacés internes. Encore devait-on attendre

5 De ce point de vue, il convient de noter que la version des déplacés diffère de celle d'analystes américains comme Ronald Asmus (2010), pour qui le président Mikheil Saakashvili aurait au contraire précipité son offensive afin de contenir les Russes et d'avoir le temps d'évacuer les Géorgiens d'Ossétie du Sud. 
jusqu'en août 1998 pour qu'un décret d'application fixe le montant des aides prévues.

Il est vrai que les déplacés d'alors ont eux-mêmes contribué à freiner les possibilités d'intégration en Géorgie. Pour des raisons de connivence culturelle, beaucoup ont ainsi préféré envoyer leurs enfants dans des écoles séparées, mises en place en 1994 sous l'égide de l'administration d'Abkhazie en exil à Tbilissi. Soucieux de préserver leur droit au retour avec un statut juridique de déplacé, ils ont par ailleurs continué d'enregistrer leur région natale comme lieu de résidence. Le résultat est qu'ils n'ont pas pu bénéficier des programmes de privatisation qui ont permis à leurs concitoyens de devenir quasiment gratuitement propriétaires de leurs logements ou de leurs champs. En Abkhazie, les terres ont été redistribuées sans eux et ils en ont été réduits à essayer d'acheter des lopins au marché libre en Géorgie. La loi de juin 1996 leur a seulement accordé un droit d'usage et non de propriété. En attendant mieux, un tel dispositif est revenu à leur interdire de construire des structures permanentes, notamment des habitations en dur.

Leur frustration n'en est donc que plus grande à l'égard des déplacés de 2008, pour qui le gouvernement a construit des maisons livrées clé en main. Des tensions sont également apparues autour des foyers collectifs où ont été accueillis les nouveaux venus. À Tbilissi, des déplacés de la première génération ont par exemple essayé de forcer les portes de l'ancien hôpital militaire d'Isani afin de profiter de l'hébergement et de l'aide fournie aux victimes de la guerre de 2008. Des frictions les ont aussi opposés à la population locale, qui les a accusés d'aggraver le chômage ou de pénaliser l'industrie touristique. La tension a notamment été perceptible autour des hôtels squattés par les déplacés de la première génération sur la côte ou dans les stations thermales de Tskaltubo et Borjomi. Parce qu'ils ont trouvé abri dans des crèches ou des écoles qui ont dû fermer leurs portes aux habitants du voisinage, les nouveaux venus n'ont pas non plus échappé au problème. Sachant les incertitudes qui pèsent sur le foncier, beaucoup de bâtiments publics réquisitionnés afin de loger les déplacés de 2008 s'avèrent en fait avoir été mis en vente ou déjà cédés à des promoteurs immobiliers qui pressent aujourd'hui le gouvernement de procéder à l'évacuation des lieux pour prendre possession de leurs biens. À en croire la rumeur, l'ancien hôpital militaire d'Isani aurait ainsi été acheté par un oligarque, Kakha Bendukidze, qui a été le maître d'œuvre de la privatisation de l'économie géorgienne.

Une telle pression immobilière a certainement poussé le gouvernement à hâter le mouvement pour reloger les déplacés d'Ossétie en dehors de la capitale et renvoyer les autres victimes du conflit dans la région de Gori. Les autorités ont préféré prendre les devants et ne pas laisser des squatters s'installer durablement dans des bâtiments vacants, au risque de compliquer les procédures d'expulsion. Elles n'ont pas voulu renouveler l'expérience du célèbre hôtel Iberia, « défiguré » et occupé pendant plus de dix ans par des déplacés de 1993 sur une des plus belles avenues de Tbilissi. Pour autant, la pression immobilière et le risque d'une explosion sociale dans la capitale n'expliquent pas tout. Très prosaïquement, la politique du gouvernement a aussi mis en évidence les dysfonctionnements de l'État géorgien. Ses contradictions, notamment : autoproclamé coordinateur de l'aide aux "réfugiés », un parlementaire influent, Koba Subeliani, a ainsi invité les déplacés à revenir chez eux alors que le gouverneur de la province de Shida Kartli, Lado Vardzelashvili, venait de mettre en garde les habitants de la région contre un retour précipité dans des villages qui n'avaient pas encore été déminés ! 
En réalité, la politique humanitaire du gouvernement a d'abord reflété les rapports de force du moment, avec un ministre de l'Intérieur qui a littéralement court-circuité les autres administrations chargées de gérer l'aide aux populations sinistrées. Statutairement, la police a certes un rôle à jouer en cas de catastrophe, par exemple à la suite d'un tremblement de terre. Mais dans les textes, il n'est nullement prévu de lui confier la supervision de la construction de nouveaux logements pour abriter les sinistrés. De même, le recensement des déplacés aurait dû revenir au ministère des Réfugiés et non aux fonctionnaires de l'état-civil, qui dépendent de la Justice. Dans l'urgence, les tâches les plus immédiates ont en fait été réparties entre les principaux responsables de l'appareil coercitif de l'État, quitte à promouvoir une vision quasi-militaire de la gestion des flux de population. Après la défaite de l'armée, le gouvernement a plutôt misé sur la police car elle lui paraissait autrement plus efficace que le ministère des Affaires sociales, de la Santé ou, surtout, des Réfugiés. Résultat, la multiplicité des centres de décision a rendu la situation pour le moins confuse. Quatre mois après la guerre d'août 2008, les autorités n'avaient toujours pas produit de plan d'action écrit et on continuait de se demander si les déplacés d'Ossétie seraient finalement autorisés à devenir propriétaires des maisons construites le long de l'autoroute de Gori.

\section{D'UN PLAN À L'AUTRE}

L'enjeu est pourtant bien de passer d'une logique d'assistance à une stratégie de réhabilitation et de développement. Les demandes de financements présentées à la communauté internationale s'inscrivent ainsi dans une perspective assez large et englobent toutes les victimes des précédentes catastrophes, qu'il s'agisse de conflits armés ou des tremblements de terre qui secouent régulièrement la région. L'objectif est de faire d'une pierre deux coups et de résoudre par la même occasion la question restée pendante des déplacés de la première génération. Ces derniers continuent en effet de vivre dans une situation précaire. Malgré l'absence de fiabilité des chiffres, on estime que $44 \%$ d'entre eux habitent toujours dans des centres collectifs (Sumbadze et Tarkhan-Mouravi, 2003 : 19). Des études montrent en outre qu'en cas de maladie, ils sont moins bien couverts par la sécurité sociale que leurs concitoyens (Zoidze et Djibuti, 2004 : 22). Il semblerait donc logique de les intégrer dans une stratégie de lutte contre la pauvreté. Pour la première fois, un plan adopté par décret en février 2007 prévoyait d'ailleurs de les intégrer définitivement en Géorgie.

Mais ce dispositif n'a jamais été mis en œuvre et la crise d'août 2008 en a repoussé l'application sine die. Là encore, des contraintes d'ordre politique ont joué. Les autorités, en l'occurrence, n'ont pas voulu regrouper les anciens et les nouveaux déplacés dans une même catégorie susceptible de donner lieu à la création d'un lobby d'opposition. Elles ont préféré dissocier les deux types de populations. Dans la terminologie officielle, les nouveaux déplacés sont ainsi qualifiés de « sans abris », comme pour mieux effacer l'origine politique de leur malheur et les neutraliser sous un vocable généraliste, sans référence aucune à la défaite militaire des Géorgiens. Les victimes des conflits de 19911993, elles, renvoient au passé post-communiste des précédents régimes au pouvoir avant la Révolution des Roses et l'élection de Mikheil Saakashvili en 2004. Le présent gouvernement reconnaît la cause militaire de leur fuite, mais ne se sent pas responsable de leurs mésaventures et s'en est donc peu préoccupé. 
Plusieurs facteurs expliquent le désintérêt des autorités en la matière. Le premier est évidemment que le gouvernement ne souhaite pas alourdir davantage le budget social de l'État. Les déplacés de la première génération ont ainsi dû continuer de se débrouiller par eux-mêmes pour refaire leur vie en Géorgie. À la différence de leurs collègues d'infortune dans des centres collectifs, ceux qui se sont insérés dans le tissu familial s'en sortent d'ailleurs mieux, avec un niveau de pauvreté moindre que la moyenne de leurs concitoyens (Yemstov et al., 1999 : 46). Malgré sa rhétorique nationaliste et sa volonté de récupérer les territoires perdus, le gouvernement n'est de toute façon plus en mesure d'entretenir l'espoir d'un retour. Il voit donc moins d'inconvénients à ce que les déplacés de la première génération s'installent définitivement en Géorgie : un choix qui paraît dominer si l'on en juge par l'évolution de la scolarisation des enfants originaires d'Abkhazie, dont la majorité va désormais dans des classes «normales » et non plus réservées aux exilés. $\mathrm{Au}$ contraire, le gouvernement a pu dissuader les déplacés de revenir chez eux afin de démontrer le caractère dictatorial et illégitime des pouvoirs sécessionnistes en place dans des territoires victimes de nettoyages ethniques ${ }^{6}$. En Abkhazie, notamment, il n'a pas voulu soutenir les 46000 Géorgiens qui se sont réinstallés dans le district frontalier de Gali, visiblement sans provoquer d'animosité puisque moins de 1500 sont repartis à l'occasion de la crise d'août 2008.

En principe, le plan de février 2007 envisageait certes un appui de Tbilissi aux déplacés revenus dans les territoires sécessionnistes. Mais il n'a jamais été appliqué et les bonnes résolutions de l'époque ont vite été démenties par le comportement des autorités. Dès avant la guerre d'août 2008, Tbilissi a ainsi multiplié les obstacles administratifs pour bloquer l'aide à destination de l'Abkhazie ou de l'Ossétie. Depuis lors, la proclamation d'indépendance des territoires sécessionnistes a encore aggravé la situation. Dorénavant, Tbilissi interdit aux ONG occidentales d'entrer en Abkhazie ou en Ossétie depuis Moscou car ce serait une manière d'entériner la partition du pays. De leur côté, les indépendantistes rechignent à accepter l'aide en provenance de Géorgie. Le chef du gouvernement ossète, Édouard Kokoity, s'est montré particulièrement inflexible dans ce domaine, plus que ses homologues abkhazes. D’une manière générale, les autorités sécessionnistes se méfient des partis pris de l'assistance internationale, qui a toujours favorisé la Géorgie au détriment des régions insurgées depuis 1993 (MacFarlane, 2000 ; Hansen, 2009).

\section{LE POTENTIEL STRATÉGIQUE DE L'AIDE}

Les contraintes politiques qui restreignent la circulation des secours ne sont finalement qu'une des nombreuses manifestations du potentiel stratégique de l'aide. Qu'il s'agisse de reconnaissance diplomatique ou de légitimation humanitaire du bon droit d'une des parties au conflit, l'assistance de la communauté internationale n'est certainement pas neutre, instrumentalisée qu'elle est par ses bailleurs et ses bénéficiaires tout à la fois. La mise en scène médiatique des victimes, en particulier, fait partie intégrante de la propagande de guerre. On l'a vu lorsque les Russes ont diffusé des témoignages en vue de

6 Il importe de noter que les Abkhazes eux-mêmes n'ont rien fait pour encourager le retour des Géorgiens, de crainte que ne s'infiltrent parmi eux des combattants susceptibles de commettre des attentats contre les autorités locales, comme en 1996-1998 dans le district de Gali. 
démontrer que les Ossètes avaient été l'objet d'un véritable génocide. Du côté géorgien, on a également essayé d'amplifier l'ampleur des destructions en regroupant artificiellement les déplacés dans des camps de tentes à Gori ou à proximité de l'aéroport de Tbilissi. En effet, les médias internationaux préfèrent généralement filmer les victimes dans des abris précaires plutôt que des centres collectifs en dur.

Au-delà de l'urgence, les enjeux stratégiques de l'aide ne s'arrêtent évidemment pas là. D'une manière générale, la transition de la reconstruction vers le développement ressort également d'une problématique politique. Sous couvert de reconstruire les infrastructures détruites pendant la guerre, l'aide de la communauté internationale a bien pour objectif d'affermir l'ancrage de la Géorgie dans le camp occidental et de confirmer la libéralisation d'une économie post-soviétique désormais acquise aux principes du marché. Les financements de l'Union européenne, des Nations Unies, des États-Unis et de la Banque mondiale sont en l'occurrence prévus pour s'étaler sur plus de trois ans et conforter les réformes entreprises par le gouvernement du président Mikheil Saakashvili. L'objectif n'est pas seulement d'assister les victimes de la guerre, mais aussi de poursuivre la modernisation d'un pays post-soviétique converti non sans mal à l'économie de marché. Les montants en jeu, qui s'élèvent à 4,5 milliards de dollars, sont conséquents pour un petit pays de 4,3 millions d'habitants. En trois ans, la Géorgie va recevoir à peu près autant qu'en dix ans après sa proclamation d'indépendance et sa sortie de l'URSS en 1991, avec une aide internationale estimée à 3,7 milliards de dollars entre 1992 et 2002 (Sumbadze et Tarkhan-Mouravi, 2003 : 46).

De la part des Occidentaux, de tels engagements ne sont évidemment pas sans arrière-pensées géopolitiques. Comme au temps de la guerre froide, ils s'inscrivent dans le cadre d'une stratégie d'endiguement face à Vladimir Poutine. Il s'agit notamment de contourner la Russie en sécurisant la filière d'exportation du pétrole d'Asie centrale à travers le Caucase du Sud. Pour les États-Unis, en particulier, la Géorgie constitue une véritable tête de pont dans la région ; avant les événements, elle était déjà, avec l'Ukraine, la principale bénéficiaire de l'aide américaine parmi les pays de la zone (Gangloff, 2008 : 47). Aujourd'hui, l'objectif est donc de marquer son appui à un gouvernement allié sans aller jusqu'à l'engagement militaire, de crainte de se retrouver entraîné dans des aventures guerrières sous l'impulsion d'un président quelque peu incontrôlable (Pérouse de Montclos, 2008). L'aide occidentale vise en quelque sorte à compenser le refus d'admettre la Géorgie au sein de l'OTAN (Organisation du Traité de l'Atlantique Nord).

De son côté, le gouvernement du président Mikheil Saakashvili veut profiter de l'occasion pour conforter son assise politique et reconstruire une légitimité mise à mal par la défaite de 2008, des élections frauduleuses et une contestation interne grandissante. Au-delà de la gestion des populations déplacées par le conflit, le défi est notamment de renouer avec la croissance économique en vue d'inciter les Abkhazes et les Ossètes à revenir dans le giron de Tbilissi. Bien que la politique de développement des autorités reste assez confuse dans les détails, l'objectif stratégique est clair. Après la défaite militaire, il s'agit de gagner la bataille des cœurs et de construire une Géorgie prospère pour attirer les travailleurs en provenance des territoires sécessionnistes : une façon de les encourager à voter avec leurs pieds, en quelque sorte... 


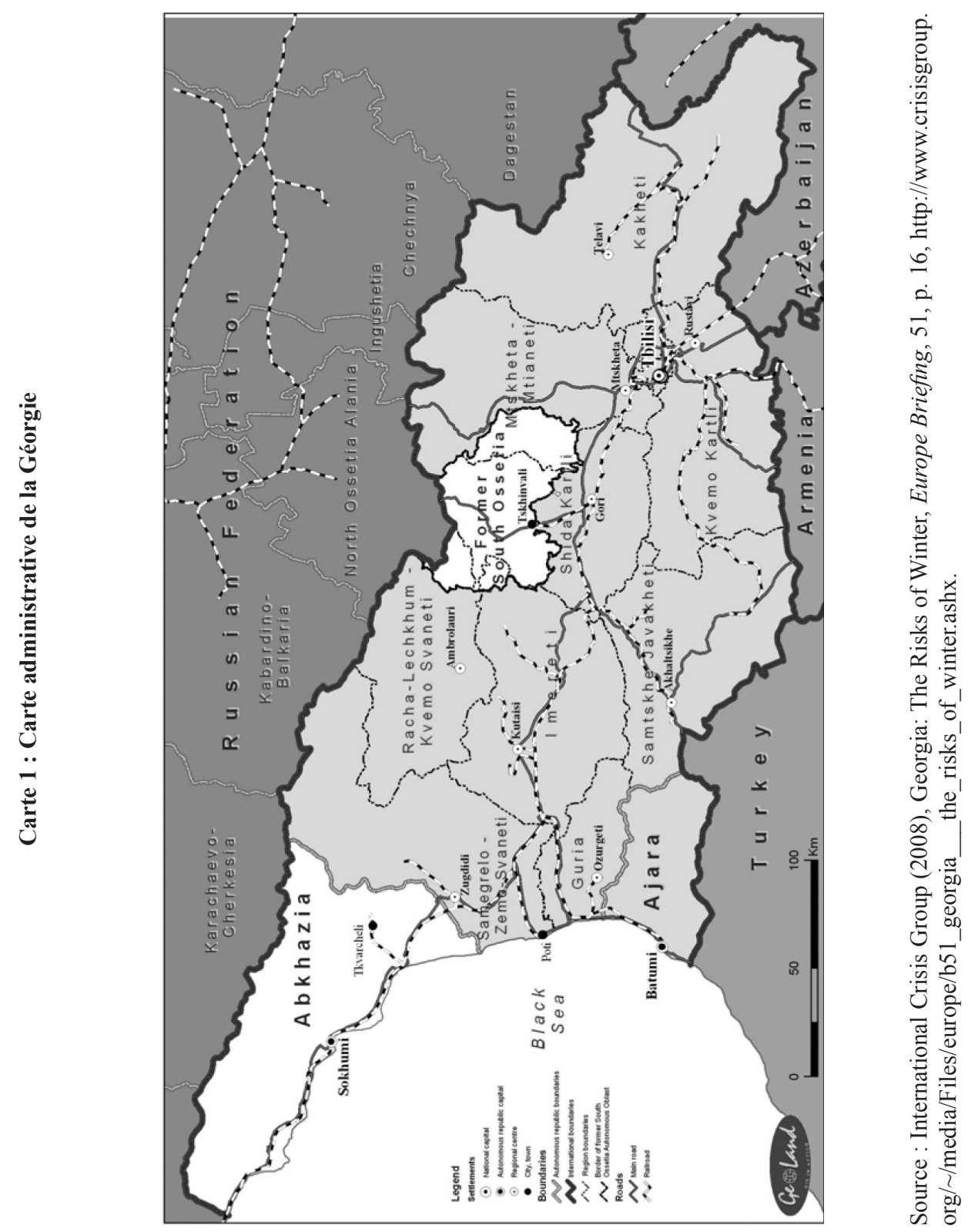




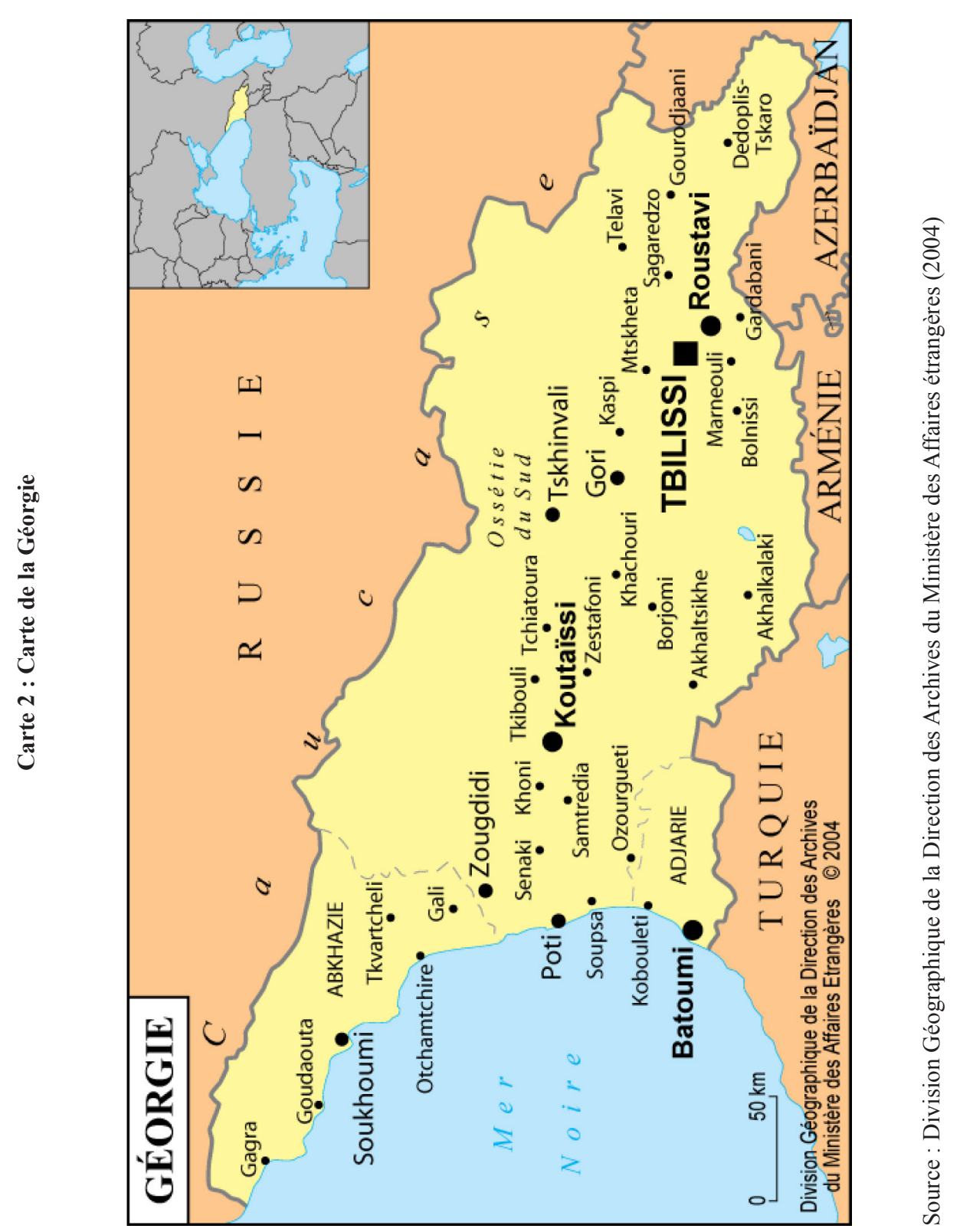




\section{Références bibliographiques}

ASMUS Ronald (2010) A little war that changed the world: Georgia, Russia, and the future of the West, New York, Palgrave Macmillan, $272 \mathrm{p}$.

B\&A (2007) Georgia National Public Opinion Survey on Remittances, EBRD (European Bank for Reconstruction and Development), London, Bendixen \& Associates, http://www.ebrd.com/ country/sector/etc/survey.htm

BAUMANN Éveline (2008) L'économie géorgienne. Menaces au présent, poids du passé, incertitudes pour l'avenir, Note 7, Paris, Fondation Jean-Jaurès, 12 p.

CAMBREZY Luc et CHARBIT Yves (Dir.) (2007) Migrations internationales et vulnérabilités, Revue Européenne des Migrations Internationales, 23 (3), pp. 7-108.

GANGLOFF Camille (2008) L'import-export de la démocratie : Serbie, Géorgie, Ukraine, Kirghizistan : ONG étrangères et mouvements étudiants, Paris, L'Harmattan.

HANSEN Greg (2009) Humanitarian Agenda 2015: Politics and Humanitarian Action in the Georgia Conflicts, Medford (MA), Tufts University, Feinstein International Center, 48 p.

KAKULIA Merab (2007) Labour migrants' Remittances to Georgia: Volume, Structure and Socio-Economic Effect, in GEPLAC, Georgian Economic Trends: Quarterly Review, Tbilissi, Georgian-European Policy and Legal Advice Centre, pp. 49-57.

KEVONIAN Dzovinar (2000) Les réfugiés et l'action humanitaire en Transcaucasie post-soviétique : enjeux politiques et considérations historiques, CEMOTI (Cahiers d'études sur la Méditerranée orientale et le monde turco-iranien), 29, pp. 65-87.

KLEINHANSS Silke (2008) Die Aussenpolitik Georgiens: ein "failing state" zwischen internem Teilversagen und externen Chancen, Berlin, Lit.

MACFARLANE Neil (2000) Humanitarian Action and Conflict in the Southern Caucasus: the Cases of Abkhazia (Georgia) and Nagorno-Karabakh (Azerbaijan), CEMOTI (Cahiers d'études sur la Méditerranée orientale et le monde turco-iranien), 29, pp. 51-64.

MINISTRY OF REFUGEES (2002) IDP's Reference Book, Tbilissi, Ministry of Refugees.

PÉROUSE DE MONTCLOS Marc-Antoine (2008), Géorgie : effacer les traces de la défaite, Libération, 7/11/2008, Paris, p. 24.

SERRANO Sylvia (2007) Géorgie : sortie d'empire, Paris, CNRS, 342 p.

SERRANO Sylvia (2008) Géorgie : le rêve brisé, Politique internationale, 121, pp. 153-172.

SHELLEY Louise, SCOTT Erik R. and LATTA Anthony (Eds.) (2007) Organized Crime and Corruption in Georgia, London, Routledge, 144 p.

STEFES Christoph H. (2006) Understanding Post-Soviet Transitions: Corruption, Collusion and Clientelism, Basingstoke, Palgrave Macmillan, $224 \mathrm{p}$.

SUMBADZE Nana and TARKHAN-MOURAVI George (2003) Working Paper on IDP Vulnerability and Economic Self-Reliance, Tbilissi, United Nations Development Programme.

WORLD BANK (2008) Georgia. Summary of Joint Needs Assessment Findings. Prepared for the Donors' Conference of October 22, 2008 in Brussels, Washington, World Bank.

YEMSTOV Ruslan et al. (1999) Georgia Poverty and Income Distribution Volume 1: Main Report, Washington, World Bank.

ZOIDZE Akaki and DJIBUTI Mamuka (2004) IDP Health Profile Review in Georgia, Tbilissi, United Nations Development Programme.

ZOURABICHVILI Salomé (2009) La tragédie géorgienne : 2003-2008, Paris, Grasset, 334 p. 


\title{
Revue européenne des migrations internationales
}

Numéro vol. 26 - n³ (2010)

Migrations en Asie centrale et au Caucase

Stéphane de Tapia

\section{Marlène LARUELLE, Dynamiques migratoires et changements sociaux en Asie centrale}

\begin{abstract}
Avertissement
Le contenu de ce site relève de la législation française sur la propriété intellectuelle et est la propriété exclusive de l'éditeur.

Les œuvres figurant sur ce site peuvent être consultées et reproduites sur un support papier ou numérique sous réserve qu'elles soient strictement réservées à un usage soit personnel, soit scientifique ou pédagogique excluant toute exploitation commerciale. La reproduction devra obligatoirement mentionner l'éditeur, le nom de la revue, l'auteur et la référence du document.

Toute autre reproduction est interdite sauf accord préalable de l'éditeur, en dehors des cas prévus par la législation en vigueur en France.
\end{abstract}

\section{revues.org}

Revues.org est un portail de revues en sciences humaines et sociales développé par le Cléo, Centre pour l'édition électronique ouverte (CNRS, EHESS, UP, UAPV).

Référence électronique

Stéphane de Tapia, «Marlène LARUELLE, Dynamiques migratoires et changements sociaux en Asie centrale », Revue européenne des migrations internationales [En ligne], vol. 26 - $n^{\circ} 3$ | 2010, mis en ligne le 03 mai 2011. URL : http://remi.revues.org/5247

DOI : en cours d'attribution

Éditeur : Association pour l'étude des migrations internationales http://remi.revues.org

http://www.revues.org

Document accessible en ligne sur : http://remi.revues.org/5247

Ce document est le fac-similé de l'édition papier.

Cet article a été téléchargé sur le portail Cairn (http://www.cairn.info).

\section{CAIRN}

Chercher. Repérer. Avancer.

Distribution électronique Cairn pour Association pour l'étude des migrations internationales et pour Revues.org (Centre pour l'édition électronique ouverte)

(C) Université de Poitiers 


\section{NOTES DE LECTURE}

LARUELLE Marlène (Dir.)

Dynamiques migratoires et changements sociaux en Asie centrale. - Paris, Petra Éditions, 2010. - 320 p. ISBN : 978-2-847-43028-8

\section{Tarlène Laruelle est avec Virginie} MSymaniec directrice aux éditions parisiennes Petra d'une collection intitulée "Sociétés et cultures postsoviétiques en mouvement " et c'est précisément dans cette collection qu'elle fait paraître un ouvrage collectif avec pour titre: Dynamiques migratoires et changements sociétaux en Asie centrale. Cet ouvrage de 320 pages n'est pas le premier de la collection écrit ou dirigé par Marlène Laruelle puisque deux titres personnels déjà y figurent : La Quête d'une identité impériale. Le néo-eurasisme dans la Russie contemporaine, paru en 2007, et avec Catherine Servant : D'une édification l'autre. Socialisme et nation dans l'espace postcommuniste, paru en 2008. Cette jeune politologue est docteure de l'Institut National des Langues et Civilisations Orientales. Elle est spécialiste du néo-eurasisme russe, sujet de son doctorat paru sous trois titres, celui de l'édition Petra cité ci-dessus, l'un paru à L'Harmattan et l'autre aux Éditions du CNRS. Elle a également signé avec Sébastien Peyrouse deux ouvrages, l'un sur les Russes du Kazakhstan chez Maisonneuve \& Larose en 2004 et l'autre chez Autrement

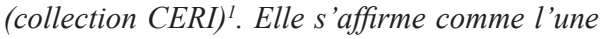
des figures montantes de l'étude des idéologies nationalistes du monde russe et ex-soviétique en philosophie politique et politologie.

1 L'Idéologie russe ou Comment penser l'empire ?, Paris, L'Harmattan, 1999 ; Mythe aryen et rêve impérial dans la Russie du $\mathrm{XXe}^{\circ}$ siècle, Paris, CNRS Éditions, 2005 ; La quête d'une identité impériale. Le néo-eurasisme dans la Russie contemporaine, Paris, Petra Éditions, 2007 ; avec PEYROUSE Sébastien, Les Russes du Kazakhstan. Identités nationales et nouveaux états dans l'espace postsoviétique, Paris, Maisonneuve \& Larose, 2004 ; Asie centrale, la dérive autoritaire. Cinq républiques entre héritage soviétique, dictature et islam, Paris, Autrement-CERI, 2006.
À ce titre, elle n'est donc pas au sens strict spécialiste des études sur le fait migratoire, mais de fait plusieurs de ses précédentes études, personnelles ou en partenariat, abordent les domaines complexes des migrations internes et internationales sur les territoires des anciennes républiques soviétiques, avec une prédilection pour l'Asie centrale au sens large, incluant le Kazakhstan. Sans être vierge, ce champ géographique de recherche reste encore mal connu du public, même relativement informé sur les tendances actuelles et récentes des flux migratoires sur l'immense territoire que représentent les quinze anciennes républiques. Deux raisons à cela, d'une part la réelle immensité des espaces considérés, rappelons que la Russie, même " esseulée ", couvre plus de 17 millions de kilomètres carrés, et le Kazakhstan, avec ses 15 millions d'habitants, plus de 2,7 millions de kilomètres carrés (soit l'équivalent à lui seul de l'UE!), immensité qui recèle des situations incroyablement diversifiées et mal connues, d'autre part, la relative nouveauté des questions liées aux mobilités et migrations dans des pays où la recherche en sciences humaines et sociales, sans être totalement absente de ces problématiques, restait dépendante de l'État central. Enfin, pourrait-on ajouter, la dislocation de l'URSS, accompagnée de quelques crises graves, mais moins nombreuses que ce que l'on aurait pu imaginer, a déclenché plusieurs mouvements massifs de mobilités forcées : Tchétchénie et Nord-Caucase, Karabagh, autour de la vallée de Fergana, conflits interethniques en Géorgie, etc. L'accident de Tchernobyl lui-même a poussé à l'exode des dizaines de milliers d'habitants et les Russes, passablement inquiets quant à l'avenir dans des républiques nouvellement indépendantes où ils n'étaient pas toujours bien vus par les autochtones " titulaires", ont souvent pris les devants en partant par " principe de précaution". Parmi les tout premiers ouvrages traitant de ces questions, on rappellera celui de Hisao Komatsu, Chika Obiya et John Schoeberlein qui 
était sans doute l'un des premiers disponibles ${ }^{2}$. Depuis les publications se sont multipliées, souvent sous les plumes d'Anatoli Vichnevsky, Jana Zayontchovkaya, Irina Ivaniukh, Vladimir Iontsev, Vladimir Mukomel, Vladimir Kolossov, etc. (Russie), Elena Sadovskaya (Kazakhstan), Saodat Olimova (Tadjikistan), etc. Les études commandées par les gouvernements républicains sur les "diasporas » se multiplient également, mais parfois dans une optique très dirigée. En d'autres termes, des "écoles" commencent à émerger en divers lieux de Russie ou dans les nouvelles capitales (Kiev, Astana-Almaty, Bishkek, Bakou). Mais il reste une grande marge de manouvre pour approcher l'état des connaissances occidentales (américaines, européennes, australiennes, japonaises, turques, inter alia) en ce domaine. La sociologie, l'anthropologie, plus que la politologie ou l'histoire restent, non pas à inventer, mais au moins à conforter tout en n'appliquant pas de façon simpliste et/ou néocoloniale des normes, méthodes et épistémologies venues d'ailleurs. L'étude des migrations a donc un avenir certain dans cette région du monde, reposant sur des faits évoluant extrêmement rapidement.

Au-delà d'une première introduction (pp. 9-20) rédigée par la coordinatrice, cet ouvrage aborde trois aspects plus spécifiques en trois parties : I. De la multiplicité des flux en Asie centrale, avec des textes d'Olivier Ferrando, Marlène Laruelle, Sophie Massot, Sébastien Peyrouse ; II. Évolutions sociales, transformations identitaires stratégies administratives, avec les contributions d'Asel Dolotkeldieva, Adeline Braux, Sophie Hohmann et Sophie Massot; enfin une partie III. L'impact des migrations sur les rapports de genre avec des articles de Madeleine Reeves, Luisa Piart, Nafisa Khusenova, Stéphanie Belouin. La première partie revient utilement sur l'historique assez méconnu en dehors des spécialistes de la Russie et de l'URSS des migrations russes et soviétiques sur le long

2 KOMATSU Hisao, OBIYA Chika et SCHOEBERLEIN John $(E d s)$ (2000), Migration in Central Asia: Its History and Current Problems, Osaka, The Japan Center for Area Studies, National Museum of Ethnology, 9, 255 p. terme et fixe les grandes lignes de mobilités en grande partie dictées par le politique plus que par l'économique, bien que, ici comme ailleurs, il soit parfois difficile de fixer des limites franches. Elle permet, avec Sébastien Peyrouse (Les anciens colons sur le départ. Les migrations des populations russophones, pp. 21-48) et Olivier Ferrando (Politiques diasporiques et flux migratoires : les États-nations et leurs co-ethniques de l'étranger, pp. 49-76) de mieux resituer les actuels courants migratoires dans un contexte plus large qui couvre les trois ères tsariste, soviétique et postsoviétique. De fait, migrations et mobilités ne sont pas nouvelles dans cet espace, mais il est resté l'image tenace d'une absence totale de liberté de déplacement hors migrations gérées par le politique, ce que viennent largement nuancer Sébastien Peyrouse et Olivier Ferrando par leurs mises au point. Au passage, remarquons l'usage d'une notion en rapide devenir, celle de "co-ethnique ", pour qualifier des populations de même langue et culture vivant dans des pays différents suite au découpage des frontières internationales. Ainsi pour ne citer qu'un exemple, les Ouzbeks présents en Kirghizie (sujet d'actualité !), Kazakhstan, Turkménistan, Russie, tous anciens citoyens soviétiques, et en Afghanistan, Chine, Turquie, Arabie saoudite et autres pays qui s'apparenteraient eux plutôt à des populations diasporiques au vu des circonstances historiques et politiques de leurs mobilités. La richesse du thème apparaît avec les textes de Marlène Laruelle (Le Kazakhstan, nouveau carrefour migratoire d'Asie migratoire, pp. 77-98) et Sophie Massot (L'exode rural comme migration de l'identité. Du kichlak ouzbek à Samakand la tadjike, pp. 99-122) montrant comment les situations se diversifient rapidement, ainsi du Kazakhstan pays d'émigration massive se retransformant en pays d'immigration, ou l'ambiguïté du cas ouzbek où les Tadjiks autochtones (descendants des sédentaires iranophones des oasis) se transforment en étranger de l'intérieur, mais où les ruraux ouzbeks finalement se " tadjikisent » en s'urbanisant, donc en porteà-faux par rapport à une politique nationale d'ouzbekisation (o'zbekchilik) qui en rappelle 
bien d'autres ${ }^{3}$.

Dans la seconde partie relative à des terrains plus localisés, reposant sur des recherches de thèses de doctorats en cours ou récemment soutenus, Asel Dolotkeldieva (Les migrants kirghizes à Moscou, politiques publiques, stratégies migratoires et réseaux associatifs, pp. 123-145, thèse en cours) nous propose une lecture d'une migration de travail peu qualifiée qui s'apparente à ce que l'Europe a connu avec les Maghrébins en France ou les IndoPakistanais en Grande-Bretagne : les droits les plus basiques des travailleurs aujourd'hui étrangers (alors qu'ils étaient des compatriotes auparavant, il n'y a pas si longtemps) ne sont guère respectés, corruption et xénophobie ont augmenté dans des proportions importantes sans que les autorités, tant des pays d'accueil et de résidence que des pays de départ, ne soient réellement en mesure de contrôler des situations encore largement inédites sur cet espace postsoviétique. Les migrants, ici précisément kirghizes, tentent de s'organiser pour à la fois se défendre et faire valoir leurs droits sur les deux bornes du champ migratoire. Adeline Braux (Les Azerbaïdjanais de Russie : une diaspora imaginée, pp. 146-168, à partir d'une recherche en cours) décrit une population presque otage des autorités de Bakou, mais face à un dispositif artificiel qui semble ne pas être efficace sur la population concernée, très passive face aux incitations du pays d'origine. L'auteur rejoint sur le cas spécifique de Moscou l'analyse un peu plus ancienne de Bayram Balci, tout aussi critique sur cette approche étatique du phénomène de diaspora ${ }^{4}$. Sophie Massot (Les migrations ouzbèkes vers Moscou, Séoul et New York: sacrifice ou rite de passage ?, pp. 169-188, thèse soutenue à Paris) nous livre un condensé d'une thèse ambitieuse et réussie sur trois terrains de recherche très éloignés mettant des migrants ouzbeks aux prises avec des réalités

3 On peut ici se référer au travail de PETRIC Boris-Mathieu (2002), Pouvoir, don et réseaux en Ouzbékistan postsoviétique, Paris, PUF-Le Monde (Partage du savoir), 298 p.

4 BALCI Bayram (2008), La place de la " diaspora 》 azerbaïdjanaise dans la politique de l'Azerbaïdjan postsoviétique : esquisse d'analyse, EurOrient, 28, pp. 185-204. économiques et sociales bien différentes. Alors que l'Ouzbékistan ne reconnaît pas la réalité du fait migratoire et l'importance de l'émigration, y compris dans son bilan économique et social, on voit ici les intéressés obligés de découvrir par leurs propres moyens des marchés très éloignés de leurs bases familiales. La question posée est celle de la migration, enjeu fondamental de la socialisation et de l'intégration sociale en Ouzbékistan. Sophie Hohmann (Migrations et enjeux socio-économiques et sanitaires de la guerre au Tadjikistan, pp. 189-216) revient sur une réalité plus dure encore, celle des suites à moyen et long terme d'un conflit violent, qui non seulement a fait de nombreuses victimes et de gros dégâts matériels, mais a aussi durablement déstabilisé une société qui était déjà l'une des plus pauvres et des plus défavorisées de l'URSS. Les aspects sanitaires (dégradation des soins et de la couverture sociale, épidémie $d u$ SIDA, etc.) sont le thème de spécialisation de l'auteur qui arpente un terrain des plus difficiles en collaboration avec les chercheurs tadjiks comme Madame Saodat Olimova.

Transition est faite par ce texte avec la partie suivante, car la population féminine $d u$ Tadjikistan est sans doute l'une des plus importantes victimes de la dislocation de l'URSS. Madeleine Reeves (Migrations, masculinité et transformations sociales dans la vallée de Sokh, pp. 217-246) et Nafisa Khusenova (La féminisation des migrations de travail tadjikes en Russie, pp. 278-296) nous décrivent des mutations importantes touchant, par le biais de l'émigration, même - et surtout! - lorsque l'épouse reste attachée au lieu d'origine. Soit elle est totalement victime de la situation nouvelle qui lui est imposée (avec le retour du patriarcat, de la polygamie), soit au contraire elle profite de nouvelles responsabilités pour transformer la société locale. Dans le cas des terrains étudiés par Luisa Piart (Des mondes marchands mobiles. Les commerçantes à la valise ouzbèkes, pp. 247-277, doctorat en cours) et Stéphanie Belouin (Projets et stratégies migratoires de femmes qualifiées à Tachkent, pp. 297-318), tous deux Ouzbeks, ce sont au contraire les femmes qui prennent l'initiative de l'émigration et viennent bousculer l'ordre 
établi des priorités et des hiérarchies. Mais dans ces deux cas, contrairement aux terrains tadjiks, les femmes bénéficient de formations et de statuts de départ bien plus enviables que les femmes du Tadjikistan rural.

Plusieurs questions transversales apparaissent ou sont posées par l'un ou l'autre article. La question des "co-ethniques " est dans l'ex-URSS une question centrale, car les frontières n'ont pas respecté - pouvaientelles d'ailleurs l'être réellement? - la répartition géographique des populations. Ceci est le thème de bien d'autres recherches en cours. Celle des "diasporas 》 en est directement issue et il faudrait réellement impulser des recherches sur ces politiques étatiques de construction de diaspora que pratiquent à des degrés divers Azerbaïdjan, Ouzbékistan ou Kazakhstan, à l'instar de la Russie. La question des migrations et droits de l'homme est cruciale, car corruption, xénophobie et nonrespects des droits parfois les plus élémentaires font dans ce vaste espace des ravages et malgré les efforts consentis par les gouvernements, au moins certains d'entre eux, les situations restent précaires, d'autant que la migration de travail a pris ici un tour inédit auquel les autorités ne sont guère habituées. Enfin, les conséquences de la migration sur les équilibres sociaux, en particulier sur les questions de genre, mériteront de vrais programmes de recherche pluridisciplinaires et sans doute internationaux.

Au total, un ouvrage frappant et passionnant par la diversité des situations étudiées, des terrains et des échelles d'analyse. Deux des originalités de cet ouvrage collectif sont la part donnée aux jeunes chercheuses en général (Adeline Braud, Sophie Hohmann, Sophie Massot, Stéphanie Belouin, Luisa Piart), et la présence de jeunes chercheuses ellesmêmes originaires de la région étudiée et très impliquée dans la relation entre la France et leurs pays respectifs (Asel Dolotkeldieva, Nafisa Khusenova). La directrice de l'ouvrage a su réunir une équipe jeune et diversifiée, l'ouvrage s'en ressent nettement et l'on comprend qu'une dynamique fondée sur une collaboration nouvelle est en train de s'affirmer sur un espace qui reste en grande partie inconnu hors des spécialistes de la région.

Stéphane de Tapia

Cultures \& Sociétés en Europe, Strasbourg Associé à MIGRINTER, Poitiers

\section{CUCHE Denys}

La notion de culture dans les sciences sociales [4e édition revue et augmentée]. — Paris : La Découverte, 2010. — 157 p. ISBN : 978-2-707-15883-3

Ceize ans après sa première publication en $\mathcal{N}$ 1994, la quatrième édition de l'ouvrage de Denys Cuche, La notion de culture dans les sciences sociales, paraît dans une version revue et augmentée (2010). Ce petit livre remarquable par son ambition synthétique, utile aux étudiant-e-s comme abondamment cité par les enseignants-chercheurs les plus confirmés, réussit le pari ô combien difficile de faire le portrait critique de l'usage de la notion de culture en sociologie et en anthropologie, en adoptant une perspective à la fois diachronique et synchronique. Rédigé dans un langage abordable, le panorama proposé expose avec complétude et rigueur les principaux enjeux et débats autour de ce mot polysémique et insaisissable, inhérent au vocabulaire des sciences sociales et humaines, en particulier dans l'étude des processus migratoires, mais pas seulement. Omniprésent dans le vocabulaire des sciences sociales, le terme a désormais aussi gagné le monde des médias, de la politique, de l'entreprise, des corporations professionnelles, du sport, de la jeunesse, de la formation, etc., autant d'univers qui se sont approprié le mot selon des logiques qui leur sont propres et qui appellent une analyse sociologique spécifique. Témoignant d'une certaine vulgarisation de l'anthropologie culturelle, l'expansion de cette notion ne date cependant pas d'aujourd'hui : il y a plus de vingt ans déjà, Marc Augé remarquait que le succès du terme de culture est à lui seul une donnée ethnologique (Augé, 1988, in Cuche, 2010 : 5). À ce titre, l'étude de l'usage de ce mot et de son appropriation par l'anthropologie et la sociologie éclairent de manière 
significative les logiques épistémologiques de ce que nous pourrions appeler - si nous ne craignions les abîmes sémantiques - nos "cultures disciplinaires ». Le triomphe du terme de culture soulève donc la question de l'intérêt d'instituer un courant de recherche plus systématisé à l'intérieur de nos disciplines autour de ce phénomène social, qui éclaire, par un double effet de miroir, les processus étudiés comme nos propres champs de recherche.

Composée de huit chapitres partiellement réorganisés, cette nouvelle édition de l'ouvrage de Denys Cuche gagne notamment en lisibilité et en clarté dans sa structure. Sur le plan formel, elle bénéficie d'une mise en page plus aérée et d'une table des matières qui inclut enfin les encadrés, au nombre de huit, qui rythment le livre et se focalisent sur une thématique ou un concept particulièrement significatifs dans l'histoire et la construction de la notion de culture en sciences sociales. Plus particulièrement, mentionnons à cet égard l'adjonction de deux nouveaux encadrés : l'un sur le concept de syncrétisme (pp. 72-73), dans lequel l'auteur retrace brièvement l'évolution du mot au cours du temps, et l'autre sur celui d'ethnicité (pp. 102-103). Longtemps cantonné au domaine de l'histoire des religions avant d'être utilisé pour caractériser différents phénomènes culturels, le concept de syncrétisme a conservé un sens négatif jusqu'au premier tiers du XXe siècle au moins. Les cultures dites "syncrétiques » désignaient alors des cultures suspectées d'impureté, contaminées par des éléments hétérogènes. Avec le courant de la postmodernité, le phénomène du syncrétisme est au contraire considéré de manière positive, célébrant le métissage et le mélange comme des buts à atteindre. Rejetant les notions de pureté ou d'authenticité culturelle, comme l'idée d'associer un jugement de valeur au phénomène du syncrétisme, l'anthropologie contemporaine, nous rappelle Denys Cuche, désigne par ce concept un phénomène universel, décrivant le processus de création d'un ensemble culturel original. Cependant, l'auteur souligne l'insuffisance du concept en lui-même pour rendre compte de la complexité des processus de métissage culturel et insiste sur l'importance d'identifier les formes de syncrétismes à l'œuvre - à travers par exemple la typologie réalisée par Bastide. Il invite aussi à procéder à une analyse fine de la situation socio-historique dans laquelle un type de syncrétisme apparaît. Dans le chapitre VI "Culture et identité », la mise en évidence du concept d'ethnicité par un encadré doit être relevée dans la mesure où sa quasi-invisibilité dans les éditions précédentes est significative de la réserve avec laquelle ce concept, " importé " de l'anthropologie anglo-saxonne, a été accueilli par l'anthropologie de langue française. Bien que l'approche relationnelle et situationnelle de l'identité et de l'ethnicité proposée par Barth (1969), abondamment cité par Denys Cuche dans ce chapitre, semble désormais avoir atteint un certain degré de consensus à l'intérieur de la discipline, il n'en demeure pas moins que l'anthropologie de langue française n'a intégré le mot " ethnicité » dans son vocabulaire d'usage courant qu'assez récemment. À cet effet, cet encadré sur le concept d'ethnicité, signé par Simon (1994), se focalise sur les ambiguités du concept en identifiant six points de flottement dans sa signification et comble une lacune à notre avis importante dans ce panorama de l'histoire et de la construction de la notion de culture dans nos disciplines. Plus globalement, la mention faite aux ouvrages de Poutignat et Streiff-Fenart (1995) et Martiniello (1995) dans la bibliographie de ce chapitre, absents dans les éditions précédentes, s'inscrit dans le même sens et contribue à une meilleure compréhension de l'articulation des concepts d'ethnicité et d'identité avec celui de culture.

Parmi les principaux ajouts à signaler dans cette édition de l'ouvrage de Denys Cuche, le plus perceptible est sans nul doute l'addition d'un nouveau chapitre sur la question des cultures d'entreprises et des cultures professionnelles (chapitre VII, pp. 115-125). Incluse à l'intérieur d'un autre chapitre dans l'édition précédente, cette partie devient ici un chapitre autonome, complété d'une partie inédite sur les grandes écoles françaises et la culture professionnelle militaire (pp. 121-125). L'auteur y retrace notamment le contexte d'émergence de la notion de "culture d'entreprise " aux 
États-Unis dans les années 1970, en France dès les années 1980, et analyse avec acuité l'appropriation de la notion anthropologique de culture par le langage du management comme une stratégie pour améliorer l'image de l'entreprise et favoriser l'identification et l'adhésion des salariés aux objectifs définis par les directions. C'est plus ou moins dans la même logique que celle du monde des entreprises que les grandes écoles françaises ont introduit dans leur vocabulaire la notion de culture afin de promouvoir à l'interne comme à l'externe une image positive de leur institution. Dans les deux cas, c'est souvent une acception réductrice, déterministe, culturaliste ou relativiste radicale de la culture qui est retenue. Un des mérites de ce chapitre est donc notamment de démontrer avec finesse, à l'aide d'exemples concrets tirés du terrain, l'instrumentalisation de la notion anthropologique de culture par les acteurs dans le but de servir leurs intérêts.

Signalons enfin l'ajout d'une partie sur la notion de "culture de pauvreté " (pp. 84-86), insérée dans le chapitre $V$, et celle d'une partie sur la question des rapports entre le développement et la culture (pp. 129-130) à l'intérieur du chapitre VIII consacré aux enjeux et aux usages sociaux de la notion de culture. Très succinctes, ces deux parties ont cependant le mérite de thématiser ces questions.

Centrée jusqu'ici sur les principales modifications apportées à l'ouvrage, cette recension serait cependant incomplète si nous ne rappelions, même très brièvement, le contenu global des différents chapitres qui composent ce livre. Le chapitre premier retrace la genèse sociale $d u$ mot en décryptant sonévolution sémantique dans la langue française et décrit les rivalités francoallemandes autour des termes de "kultur 》 et de civilisation au XIXe siècle. Consacrés à l'invention et au triomphe du concept scientifique de culture, les chapitres deux et trois décrivent les principaux courants et auteurs ayant contribué à son approfondissement théorique (Tylor et la conception universaliste, Boas et la conception particulariste, Durkheim et les fondateurs de l'ethnologie française, Malinowsky et le courant fonctionnaliste, l'école "culture et personnalité », Lévi-Strauss et son analyse structuraliste, etc.). Centré sur le renouvellement profond du concept de culture lié à l'analyse des processus d'acculturation, le quatrième chapitre met particulièrement en évidence l'apport des travaux de Bastide. Les recherches sur les processus d'acculturation ont permis de dépasser une conception figée et homogène de la culture et conduit à une définition dynamique de cette notion ; à ce titre, ce chapitre nous paraît particulièrement essentiel. Dans le chapitre suivant, l'ouvrage analyse les hiérarchies sociales et culturelles au cour des rapports sociaux entre cultures et au sein des cultures et fait appel aux travaux de Marx, Weber, Lewis, Grignon et Passeron, de Certeau, Morin, Appadurai et Bourdieu, pour ne citer que les plus connus. Le rapport entre le concept de culture et celui d'identité est clarifié dans le chapitre VI, et les conceptions objectivistes, subjectivistes et relationnelles de l'identité culturelle y sont notamment analysées. Comme précisé plus haut, le chapitre VII est consacré aux cultures d'entreprises et aux cultures professionnelles et enfin, le dernier chapitre traite de quelques enjeux et usages sociaux de la notion de culture. L'auteur y aborde notamment la notion de "culture politique", la question du multiculturalisme et analyse de manière critique les notions de "cultures des immigrés » et «cultures d'origine ».

Plus que des détails de forme, la réorganisation partielle des chapitres et des parties qui composent cette nouvelle édition de l'ouvrage de Denys Cuche ainsi que les ajouts introduits reflètent l'extension de la notion anthropologique de culture à de nouveaux domaines de la vie sociale, ainsi que l'importance prise par certaines thématiques au sein des sciences sociales. En ce sens, la structure du livre et sa métamorphose progressive au fil des éditions nous renseignent, en soi, sur l'évolution constante de l'usage du terme de culture à l'intérieur de nos disciplines et champs de recherche. À ce titre, bien que l'auteur assume son choix (p. 8), l'absence d'une analyse de l'usage de la notion de culture dans le champ de la sociologie de l'art et de la création se fait remarquer. De même, il est surprenant que la 
sociologie de Claude Dubar sur les identités professionnelles et le processus d'acquisition de la culture qu'est la socialisation ne soit pas mentionnée dans cette édition accordant une place significative aux cultures professionnelles et aux sciences sociales de langue française. Enfin, un éclairage sur les usages de la notion d'" interculturalité ", un terme ayant gagné depuis les années 1970 le vocabulaire de la recherche en sciences sociales, celui du monde politique, de la formation et de la santé, aurait été, à notre avis, particulièrement pertinent. Mais il est vrai que l'ouvrage de Denys Cuche ne vise pas à l'exhaustivité et à juste titre; il serait sinon appelé à être constamment complété et renouvelé tant la notion de culture est, comme le processus qu'elle décrit, dynamique et en constante reconstruction. Sans aucun doute cependant, ce petit livre centré sur les principaux acquis de l'analyse culturelle propose en 157 pages un horizon complet, rigoureux et clair de la question abordée. L'ouvrage se lit et se relit aisément et peut sans conteste être considéré comme un manuel de référence pour les sciences sociales de langue française, un " classique » dans son genre.

Anahy Gajardo

Université de Genève, FPSE

FILHOL Emmanuel et HUBERT MarieChristine

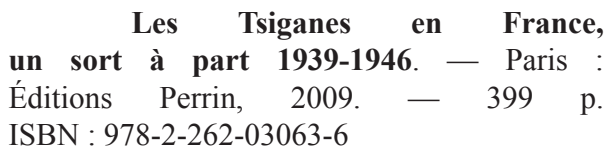

Dans un paysage où les recherches sur le sort subi par les Tsiganes pendant la Seconde Guerre mondiale sont encore émergentes en France, à la différence de pays comme l'Allemagne où l'historiographie du phénomène est plus ancienne et plus complète, ce livre foisonnant apporte une contribution importante.

L'internement a été le sort de 6000 à 6500 Tsiganes français, confinés dans trente camps d'internement pour " nomades", de 1940 à
1946. L'un des apports d'Emmanuel Filhol et de Marie-Christine Hubert est de montrer à quel point les persécutions des Tsiganes en France pendant cette période s'inscrivent dans une continuité historique débordant largement les dates de la Seconde Guerre mondiale. Le livre fait en particulier apparaître le poids des mesures législatives et administratives discriminatoires adoptées dans la première moitié du XXe siècle sur la mise en œuvre de l'internement. Ces dispositifs créent une catégorie de citoyens de seconde zone avec notamment la mise en place, en 1912, du carnet anthropométrique pour les nomades, instrument de surveillance qui servira ultérieurement aux autorités dans l'organisation de l'enfermement à partir de 1940. Ainsi, l'internement a été facilité par la suspicion d'office que les autorités publiques françaises entretenaient à l'égard des Tsiganes, suspicion qui leur a permis de passer très vite de l'omniprésence de l'idée de l'enfermement à sa mise en ouvre effective par les préfets, à qui revenaient l'organisation des interpellations et la gestion des camps. D'autres filiations historiques de la mise à l'écart des Tsiganes sont retracées : l'on découvre ainsi, par exemple, comment certains camps destinés aux réfugiés espagnols dans les années 1930 sont devenus des camps d'internement des Tsiganes quelques années plus tard, ce qui rejoint les constats des sciences sociales sur la permanence de la forme " camp » au XXe siècle.

Si la décision de l'internement appartint aux autorités militaires allemandes, son application fut entièrement assumée par l'administration française : d'ailleurs les préfets ne firent quasiment référence qu'à la loi française pour la mettre en ouvre. E. Filhol et M.-C. Hubert explorent à travers une minutieuse recherche des archives la manière dont l'internement a été orchestré, notamment à l'échelle régionale et locale. Le quotidien de ces camps, marqué par l'abandon, les privations extrêmes, et une surmortalité, notamment des enfants, est ainsi restitué dans ses détails. L'utilisation des archives donne des aperçus saisissants des destinées individuelles des internés et de leur diversité à travers des extraits de lettres des internés ou des membres de l'administration. 
Même si la mise en cuvre de l'enfermement fut parfois chaotique, sa logique sous-jacente, celle $d u$ regroupement, de la relégation avec parfois l'idée de rééducation autoritaire par le travail, a cuvré de manière puissante, condamnant les Tsiganes à vivre, avec des variations régionales et locales, une même réalité de l'internement. L'aspect familial de l'internement fut aussi d'importance, car cela revint à une prise en otage des familles et à une relégation d'autant plus complète que celles-ci ne purent espérer aucune aide extérieure.

Dans une Europe où les Tsiganes ont de manière générale subi les persécutions et l'extermination par les régimes nazis et leurs alliés, les spécificités du cas français sont dûment soulignées. Tout d'abord, l'internement des Tsiganes concerne, dans sa grande majorité, des citoyens français. Les lettres que les internés envoient aux préfets attestent de la présence des Tsiganes français dans les rangs de l'armée française pendant la Première Guerre mondiale, et des aspirations des internés à la citoyenneté et aux droits qui leur sont niés. Autre particularité française importante, l'internement de cette population ne vise pas explicitement l'extermination (les Tsiganes du Nord de la France, zone sous commandement militaire belge, seront, eux, déportés à Auschwitz et exterminés), mais bien la mise à l'écart. On retrouve aussi dans les documents émanant des autorités une volonté de sédentarisation ou de "rééducation par le travail » dont on se demande dans quelle mesure elle traduit les intentions ultimes des autorités. Enfin, dernière spécificité : les derniers Tsiganes durent attendre 1946 pour être libérés. Ils ne bénéficièrent d'aucune aide à leur sortie des camps et n'eurent de la part de l'État français aucune reconnaissance de leur sort après la libération. Dans les décennies suivantes, la loi ne récusa pas l'objectif de sédentarisation des Tsiganes. Le souvenir des camps fut "refoulé " par l'État, mais aussi dans l'aménagement urbain des communes, et, encore aujourd'hui, rares sont les marques commémoratives attestant des faits relatés dans ce livre.
À la suite de cet exposé édifiant, plusieurs questions demeurent, dont certaines sont évoquées en conclusion sans recevoir de réponse définitive. Comment comprendre le mélange de répression et d'abandon qui caractérise ce "sort à part 》 des Tsiganes en France, internés dans des conditions indignes, sans cependant qu'ils subissent une déportation massive? L'occupant allemand a délégué le sort des Tsiganes aux autorités françaises. Celles-ci, par " un enchevêtrement de circonstances ", notamment le fait que les moyens répressifs étaient monopolisés par la déportation des juifs, n'ont pas accordé de priorité à l'extermination des Tsiganes (pp. 350-352). Comment expliquer le caractère en apparence aléatoire de l'internement, puisque le livre nous apprend que les trois quarts des Tsiganes français et de nombreux Tsiganes étrangers $y$ échappèrent, et que certains internés pouvaient quitter les camps s'ils présentaient des garanties de "sédentarité »? La réponse semble être du côté de la spécificité de la catégorisation des Tsiganes par l'administration française, puisque la catégorie de "nomade" - et non la catégorisation raciale utilisée par les nazis en Allemagne pour définir les Tsiganes - a été retenue comme base pour l'identification et l'internement de cette population. Catégorie aux contours flous, car elle a aussi servi de base à l'internement de populations comme les forains ou les sans-abri, débordant ainsi le groupe initialement visé.

La manière dont la logique exterminatrice de la politique raciale allemande et celle d'exclusion et de refus du "nomadisme " de l'État français se sont imbriquées fait jaillir de nombreux questionnements, et le travail d'Emmanuel Filhol et de Marie-Christine Hubert ne manquera sans doute pas de stimuler les futures recherches.

Alexandra Nacu Centre de Sociologie des Organisations CNRS - Sciences-Po 
GUÉRIN-PACE France et FILIPPOVA Elena (Dir.)

Ces lieux qui nous habitent.
Identité des territoires, territoires des
identités. - Paris, La Tour d'Aigues,
Ined-éditions de l'Aube, 2008. - 276 p. ISBN : 978-2-752-60494-7

Doursuivant le dialogue initié en juin 2007 à l'INED entre géographes, démographes, sociologues, anthropologues et historiens, l'ouvrage revient sur la pertinence, en ce début de XXIe siècle, du lien entre les constructions identitaires individuelles ou collectives et leurs inscriptions spatiales. Dans un contexte mondialisé où l'individu multiplie le plus souvent ses appartenances et revisite les contraintes de l'espace-temps, il est en effet légitime de s'interroger sur la capacité d'affiliation et d'identification du territoire. Tentant de répondre à cette vaste question, les auteurs placent l'individu au cour de leur réflexion et décryptent des modalités par lesquelles certains lieux sont "investis " au gré des parcours de vie. Et chacune des quinze réflexions met en évidence la pluralité des configurations et des usages possibles du territoire. En réalité, par la diversité même des terrains abordés, des échelles géographiques et des méthodologies retenues, ces textes témoignent de la vigueur du territoire comme vecteur identitaire et de sa prégnance, tant dans des discours institutionnels qu'individuels. Ainsi posé, le débat sur "ce qui fonde le sentiment d'appartenance à un territoire ", pour reprendre les termes des directrices de l'ouvrage, dépasse avec bonheur le seul champ géographique.

Cependant, la diversité des études de cas présentées, qui constitue certainement une des qualités de l'ouvrage, aurait pu permettre de retravailler en profondeur les notions de territoire et de territorialité. La notion de lieu est, quant à elle peut mobilisée. Or, si le format " actes de colloque " de l'ouvrage justifie le choix transversal d'un angle descriptif, on peut toutefois regretter une discussion théorique plus étoffée sur ces notions, certes, mouvantes. Pour autant, cet ouvrage collectif n'est pas dépourvu de ligne générale : celle de l'auscultation des mécanismes de l'appropriation spatiale, d'un territoire considéré à travers le prisme des pratiques et des connaissances. Et tous les auteurs privilégient la piste de l'identité comme construction et résultat d'un processus relationnel plutôt que comme identité catégorielle nécessairement figée. Ils sont ainsi plusieurs à interroger les discours identitaires et les valeurs qu'ils mobilisent; quand d'autres examinent le territoire par les pratiques individuelles et collectives. Ces interrogations sur " la composante spatiale des identités " (première partie) et sur "l'invention des territoires identitaires" (deuxième partie) structurent l'ensemble de l'ouvrage et se résolvent dans la reconnaissance d'une relation complexe entre identités et territoires (troisième partie).

Ces intitulés de partie reflètent d'ailleurs l'intrication inévitable de la géographie et du social dans toute tentative de définition des lieux qui nous habitent. Ouvrant les débats les coordinatrices proposent une réflexion sur le processus de " mise en place des appartenances territoriales ». Leur approche est à la fois géographique et psychosociologique : elles s'interrogent sur l'essence des lieux d'attachement et cherchent à mettre en évidence la nature du lien qui attache un individu à des lieux. Leur analyse croise l'enquête nationale de l'INSEE réalisée en 2003 sur la construction des identités (l'enquête Histoire de vie) et leurs propres entretiens réalisés dans différentes régions françaises. Émaillant largement leur article par des extraits d'entretiens, elles veulent illustrer comment les contours $d u$ " chez-soi » varient d'une personne à l'autre et selon les circonstances. Cependant, et de manière peut-être un peu contradictoire, leur étude tend à montrer qu'en dépit des parcours, il n'existe qu'un nombre limité de "modèles d'appropriation spatiale».

Yannick Sencébé prolonge cette question de l'appartenance territoriale par un texte bref et bien écrit sur l'association couramment faite entre Modernité et "déclin de l'appartenance en tant que lien social collectif et durable ». En jouant, dans le titre de son article, sur déclindéclinaison, la sociologue suggère d'ailleurs 
que ce qui apparaît comme un affaiblissement des appartenances n'est peut-être en réalité que la multiplication des modalités de liens aux lieux et aux groupes. Appartenance et territoire sont donc les pierres angulaires d'une réflexion qui fait l'hypothèse de logiques d'appartenance variable d'une part à l'espace de l'inscription spatiale (l'espace des lieux) et d'autre part à celui des flux. L'auteur en dénombre quatre: selon que les lieux d'appartenance font liens, que les liens d'appartenance font lieux, que les liens et les lieux d'appartenance sont dissociés ou que, finalement, le présent définit les lieux et les liens d'appartenance. Disant cela, l'auteur souligne à quel point mobilité et ancrage (un thème cher à M. Hirschhorn et J.-M. Bertholot) fondent, aujourd'hui, des territoires en tension entre " activisme territorial actuel » et régime de mobilité.

Si c'est toujours le lien spatial que MarieLaetitia Helluy-des-Robert aborde dans son article, elle en traite quant à elle par ces différents "degrés». Ce qu'elle qualifie d'attachement spatial d'une personne à un espace en est le premier. Mais la relation au lieu peut être plus fortement chargée d'affect et l'attachement spatial fait place à de "l'appropriation spatiale ». Les lieux deviennent alors une source d'inspiration qui "colore " la vie des individus, par opposition, probablement, à un espace indéfinissable et indistinct. Au-delà, le lieu signifiant est incorporé et devient un territoire qui " code l'identité, statutaire ou intime, des individus qui s'en réclament». L'auteur repose toutefois la question de la force $d u$ terme même ; celle d'un mot qui n'appartient le plus souvent qu'à un vocabulaire scientifique qui veut rompre avec le sens commun. Cette question traverse également, bien que d'une autre manière, l'article de Catherine Armanet sur le lieu de sépulture, et en particulier la sépulture de cendres. La géographe propose en effet le lieu de sépulture comme un critère tangible et révélateur de l'identité territoriale. Comme marqueur ou énoncé de l'attachement au lieu, celui-ci est, il est vrai, à de nombreux égards, définitif. Nous interrogeant sur la pertinence de la sépulture à traduire l'identité territoriale, l'auteur propose de fait une réflexion sur ce qu'est l'identité territoriale. Si la sépulture, et en particulier la sépulture de cendres, représente un cas limite de la réflexion sur l'identité territoriale - il peut à la fois nier toute expression identitaire spatiale ou, au contraire, affirmer un attachement territorial précis (par la dispersion ou l'inhumation de cendres dans un lieu particulier) -, l'auteur voit dans l'augmentation croissante de choix de lieux particuliers pour déposer les cendres funéraires la diversification de l'expression de la pluralisation de l'identité territoriale.

De cet article à celui de Vincent Veschambre sur le patrimoine il n'y a étonnamment qu'un pas et c'est d'ailleurs un autre intérêt de cet ouvrage. Venant clore la partie sur "la composante spatiale des identités ", l'auteur discute la notion d'identité et explicite le choix de lui substituer des termes tels que "construction identitaire » et « identification ». Il « entre» par le patrimoine pour réfléchir aux processus de construction identitaire qui permet notamment d'articuler les deux dimensions fondamentales que constituent l'espace et le temps. Le patrimoine constitue en effet un support privilégié des constructions identitaires dans la durée et il constate que le recours à ce dernier est exacerbé "dans des sociétés où la mobilité, extrêmement valorisée dans une économie flexibilisée " concerne une part importante de la population. Il conclut par une mise en garde contre la réduction des processus d'identification aux seules «identités territoriales ».

C'est à Julien Aldhuy que revient d'ouvrir la seconde partie de l'ouvrage avec son article sur les liens qui se nouent entre l'identité du territoire landais et le département des Landes. L'auteur propose le couple "identité territorialité " plutôt que celui d'"identité territoire ». En effet, pour cet auteur comme pour de nombreux géographes, le territoire n'est qu'une des modalités de cette relation. L'article de Ronan Le Coadic, qui étudie le lien territoire identité en Bretagne montre comment de "la première modernité » (XIXe et première moitié du XXe) à nos jours l'identité individuelle ou collective est une construction évolutive inscrite dans un contexte socio-historique d'interac- 
tions multiples. Il part de la création de la carte d'identité, et avec elle du territoire national, pour discuter de "l'identité à la carte et des territoires superposés » de la période contemporaine. L'auteur conclut son article en notant que les individus disposent aujourd'hui d'un vaste éventail d'identifications au territoire et mêlent, voire superposent, les appartenances. Dépassant cependant l'idée de désordre contemporain, il tente la distinction des différents territoires d'identification selon qu'ils sont des territoires légitimes pour les régions administratives et d'aménagement du territoire imposés par les pouvoirs publics ; des territoires qui résistent pour les espaces qui véhiculent les rémanences de valeurs pré-modernes et notamment religieuses; des territoires de projet portés par des projets collectifs ou individuels.

L'exemple de la Costa Serena proposé par Philippe Pesteil se situe au croisement de ces différents ensembles. Dans le contexte corse de réaménagement territorial en micro-régions, il cherche à savoir quel peut être l'impact sur les populations de la superposition de découpages territoriaux "hérités, construits en ou construction ». Il se demande si les nouveaux espaces sont véritablement vécus et appropriés par les populations ou s'il ne s'agit que d'une grille technique d'aménagement du territoire. Sylvie Sagnes dans son article sur la tentative de rebaptiser la région Languedoc-Roussillon en "Septimanie », à l'arrivée d'un nouvel élu à la tête du conseil régional (2004) est assez proche. Elle retrace l'essor contemporain de cette notion - la Septimanie - et nous fait voir les intérêts divergents de ses promoteurs. Avec cette "tentative de greffe toponymique" ratée puisqu'un an seulement après sa proposition, elle doit être retirée, face aux différentes protestations, l'auteure souligne comment cet essai de mutation géonymique participe d'un phénomène général de "retour au local " souvent traduit par des "recyclages et créations onomastiques ».

Dans l'article d'Éric Auburtin, il est également question de construction régionale puisqu'il traite de la construction d'une identité transfrontalière dans la Grande Région
SarLorLux, sarroise, lorraine et luxembourgeoise. Il nous conduit au cour de l'action administrative et politique dans la construction territoriale. Cependant, si la construction de l'Europe passe par la production de " nouveaux objets territoriaux transfrontaliers ", l'auteur rappelle que la réalité transfrontalière est également faite de la mobilisation d'acteurs économiques et associatifs. Considérant l'espace transfrontalier comme un espace vécu, il cherche à comprendre le rôle de la frontière dans la territorialité d'individus "à la fois résidents et passeurs d'une frontière ». Observant l'usage que font les travailleurs lorrains au Luxembourg, et la ressource, avant tout professionnelle, qu'elle peut constituer, il propose d'envisager la frontière comme un " ourlet » qui s'étend en fonction de l'influence des métropoles frontalières sur leurs espaces périphériques.

C'est sur l'articulation des identités et des territoires que se conclut l'ouvrage. L'article d'Hervé Le Bras traite de la question de l'ethnicité et de l'intolérance dans les relations de voisinage. Il reprend le modèle de l'économiste Thomas Schelling selon qui de faibles intolérances entre groupes au sein d'une même agglomération suffisent à produire de la ségrégation spatiale. L'auteur prolonge ce modèle en donnant à la fois raison et tort à Schilling en montrant que "de faibles intolérances produisent presque toujours des structures fortes", mais que ces structures ne peuvent être interprétées de manière univoque. Yves Guermond dans son article "L'identité nationale en question" souligne à son tour que la délimitation conduit à l'édification de frontières qui ensuite institutionnalisent les identités. Il propose une réflexion sur une identité nationale " en contrepoint des "géographies des frontières" " qui ne soit pas fondée sur "la fatalité d'un découpage fixe des "États-nations" ". Ces deux articles se font donc écho en rappelant que la frontière et sa naturalisation participent largement de l'institution du groupe.

Kamel Kateb revient sur l'instauration, en 1882, de l'état civil colonial par la France en Algérie. Il explique comment l'obligation 
d'enregistrement à l'état civil selon le système français d'identification des personnes et leur ancrage subséquent au territoire a aussi été une vaste action politique de "désancrage" de populations tribales de leur territoire. Par la sédentarisation des populations nomades, $c$ 'est en réalité la rupture du lien entre population et territoire ancestral que le gouvernement français semble vouloir opérer. En effet, pour sortir la propriété " du cadre collectif dans lequel elle était enserrée, il était nécessaire de dégager la population de l'organisation sociale constituée par la tribu ». Mais en transformant le référentiel identitaire des indigènes algériens, l'administration française a également ouvert la voie d'une "identité plus globale de type national ».

Dans ce système à dominante sédentaire qui est le nôtre, et malgré les déclarations de fin du territoire et de la territorialité de certains théoriciens de la globalisation, on ne peut ignorer que le signe de reconnaissance d'identités "propres " continue d'être en relation avec la possession d'un territoire. Christian Giordano rappelle à cet égard que le "nouveau » régionalisme européen n'a pas évacué la dimension identitaire du territoire. Pourtant, dans les sociétés postcoloniales, et dans des constellations historico-sociales bien définies, le facteur territorial peut jouer un rôle moins important dans la fabrication des identités. Sur l'île de Pulau Penang en Malaisie, dans la ville de George Town par exemple, chaque groupe possède "ses institutions et ses structures à l'intérieur d'un territoire bien défini ». La subdivision voulue par les Britanniques en quartiers et en espaces sociaux ethniques a en effet favorisé l'émergence d'identités fortement territorialisées qui s'expriment pourtant aujourd'hui par des représentations et des discours publics de "l'unité dans la diversité ", tant au niveau national que local. Le cas de Penang et de la Malaisie montrerait que, au contraire des contextes européen et nord-américain où la territorialisation de l'appartenance est souvent considérée comme la condition d'une cohabitation multiculturelle pacifique, la déterritorialisation des affiliations ethnoculturelles sans l'effacement des frontières communautaires a favorisé la cohésion sociale en créant des rapports interethniques multiples.

Le dernier article de l'ouvrage porte sur le quartier du Marais à Paris et sa représentation comme espace communautaire gay et clos. Marianne Blidon aborde ce qu'elle analyse comme un décalage entre discours (anticommunautariste) et perceptions (variées) de cet espace et de sa dimension identitaire. Elle propose une géographie du Marais à la fois plus sélective et mieux intégrée dans la ville et interroge la pertinence du couple "identité " et " espace" : aborder le Marais comme un quartier gay, c'est, nous dit-elle, ne pas voir la pluralité de cet espace. L'auteure s'attache à montrer que l'attrait du quartier se joue surtout dans la juxtaposition de communautés, au cœur d'un espace historique à l'architecture exceptionnelle.

Pour conclure la présentation de cet ouvrage à recommander aux lecteurs, il nous semble que, l'effort de clarification et de catégorisation sur les liens entre identification et territorialité, qui a retenu les auteurs, aurait gagné en force en intégrant une synthèse de l'ensemble des développements proposés. Le questionnement qui traverse ce livre sur la place des lieux dans nos constructions identitaires et territoriales, individuelles et collectives n'en aurait certes pas été épuisé pour autant, mais il en aurait peut-être été plus nourri, dans un contexte mondialisé où les lieux continuent, à n'en pas douter, de nous habiter.

Caroline Rozenholc

Migrinter, Université de Poitiers

MAZZELLA Sylvie (Dir.)

La mondialisation étudiante.

Le Maghreb entre Nord et Sud.

- Paris, Karthala, 2009. - 404 p.

ISBN : 978-2-8111-0307-1

L'ouvrage dirigé par Sylvie Mazzella de recherche intitulé Étudiants étrangers au Maghreb et dans l'espace euro-méditerra- 